\title{
ARCHIVAL DIGITIZATION FOR NON-PROFESSIONALS
}

\author{
by
}

\author{
MIA TORRES \\ Bachelor of Fine Arts, New York University, 2011
}

\author{
A thesis \\ presented to Ryerson University \\ in partial fulfillment of the \\ requirements for the degree of \\ Master of Arts \\ in the program of
}

Film and Photography Preservation and Collections Management

Toronto, Ontario, Canada, 2018

(C) Mia Torres 2018 


\section{AUTHOR'S DECLARATION FOR ELECTRONIC SUBMISSION OF A THESIS}

I hereby declare that I am the sole author of this thesis. This is a true copy of the thesis, including any required final revisions, as accepted by my examiners.

I authorize Ryerson University to lend this thesis to other institutions or individuals for the purpose of scholarly research.

I further authorize Ryerson University to reproduce this thesis by photocopying or by other means, in total or in part, at the request of other institutions or individuals for the purpose of scholarly research.

I understand that my thesis may be made electronically available to the public. 


\begin{abstract}
Archival Digitization for Non-Professionals

Mia Torres

Master of Arts

Film and Photography Preservation and Collections Management, Ryerson University 2018

This thesis provides illustrated instructions, explanations, and suggestions regarding the digitization of personal family archives at home. It discusses philosophical approaches to and implications of digitization, as well as the disparity between the collections of museums and the personal documents and artifacts found within family collections around the world. Members of the Ontario Genealogical Society, who represent the demographic for which this thesis is intended, were surveyed anonymously to understand their needs, experience levels, and concerns regarding archival digitization. This thesis demonstrates that it is still possible to digitize materials using policies and procedures that adhere to nationally-approved guidelines with the limited resources one might have at home. It provides a bridge between what has largely been a professional field and those individuals who, although not formally trained, have personal collections that they would like to digitize and preserve. This thesis divided into four sections: Preparation, Digitization, Post-Processing, and Appendices.
\end{abstract}




\section{ACKNOWLEDGEMENTS}

I would like to express my sincere gratitude to all those who have supported me throughout my academic journey:

To Jeffrey Martz for inspiring and cultivating my love of photography; To Wafaa Bilaal, Shelley Rice, Tom Drysdale, Lorie Novak, and Deb Willis for providing their endless wisdom and artistic criticism; To Sasha Gulish for entrusting me with immense responsibility and always encouraging me to pursue what makes me happy.

To Marta Braun, Thierry Gervais, Robert Burley, Dee Psaila, Mike Robinson, and all the FPPCM faculty and staff for their tireless dedication to creating a unique and exceptional degree program. To my first reader, Alison Skyrme, for her invaluable guidance and support during the development of this thesis.

To Peter Taylor, Coral Harkies, Steve Fulton, and David Thompson of the Ontario Genealogical Society for support, enthusiasm, impromptu Canadian history lessons, and for giving me full access to their treasure trove of archival materials and technological equipment during my research.

To my parents, for instilling in me a love of learning, self-education, and exploration, and for never questioning whether or not a career in photography was a wise decision; To Devon Forbes for his unwavering confidence in me, and to Idit Kohan-Harpaz for her friendship, advice, and endless supply of pasta Bolognese. 


\section{TABLE OF CONTENTS}

AUTHOR'S DECLARATION FOR ELECTRONIC SUBMISSION OF A THESIS ..............ii

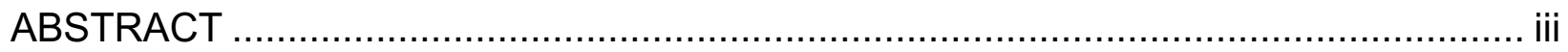

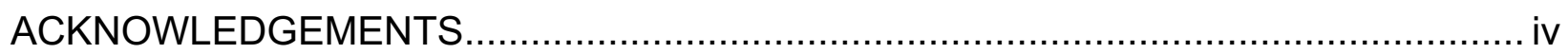

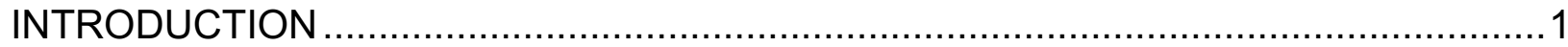

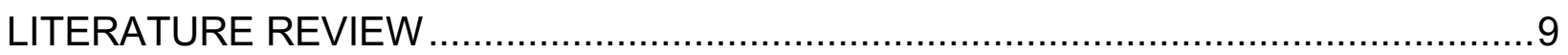

ARCHIVAL DIGITIZATION FOR NON-PROFESSIONALS ....................................21

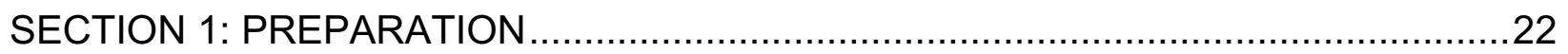

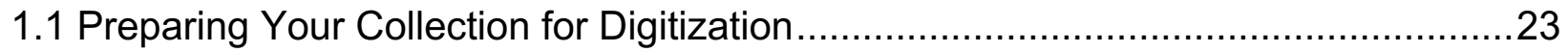

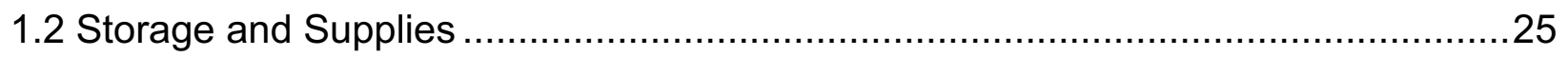

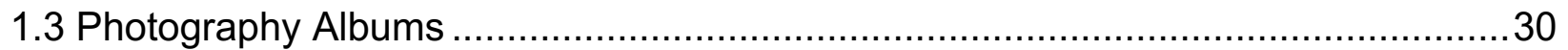

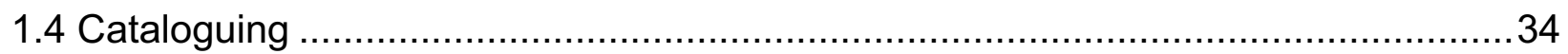

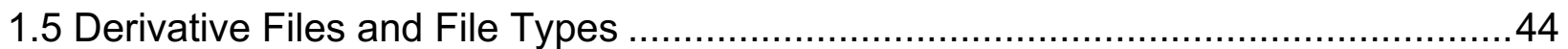

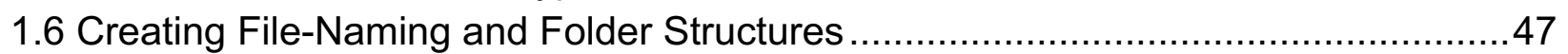

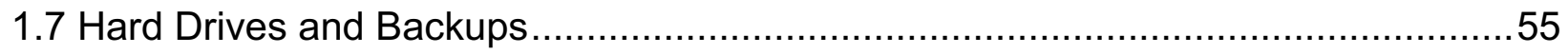

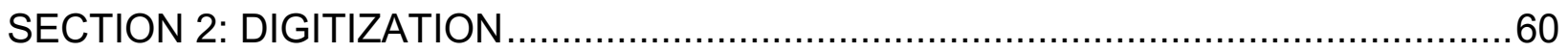

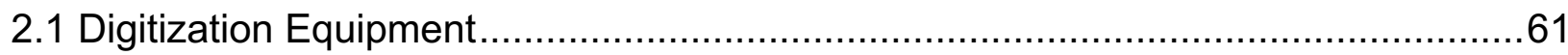

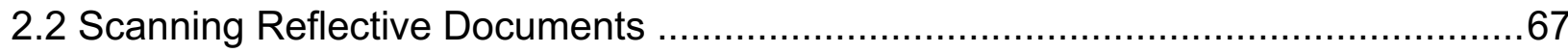

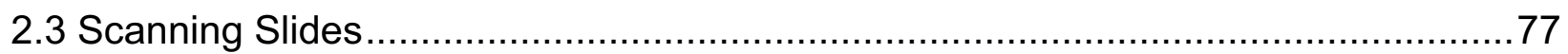

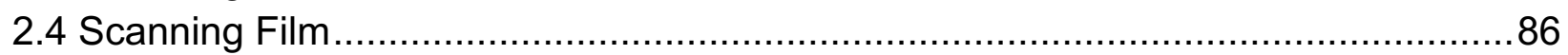

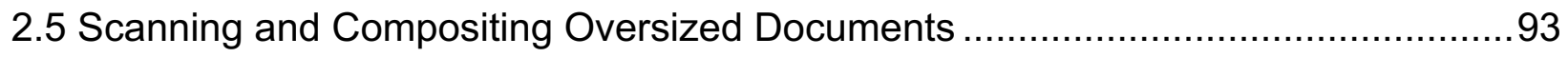

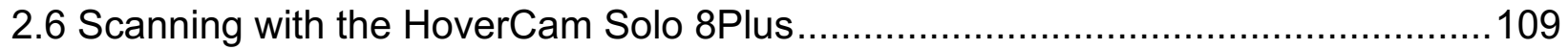

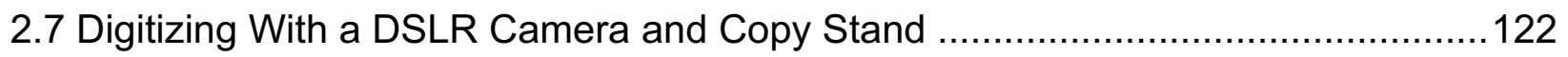

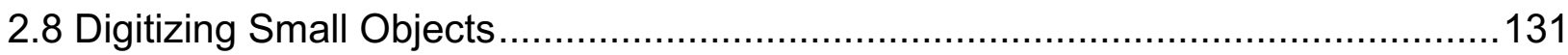

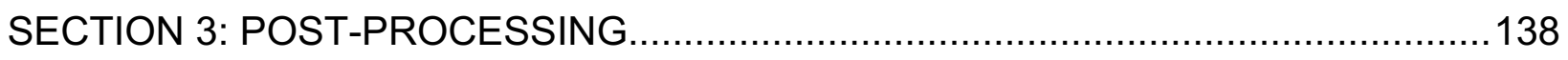

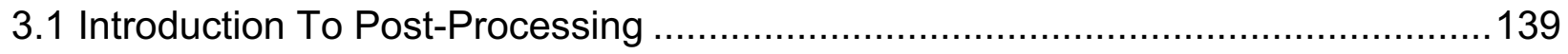

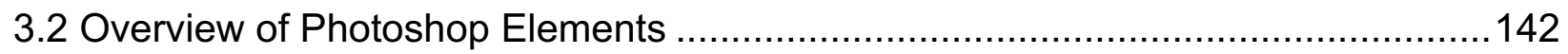

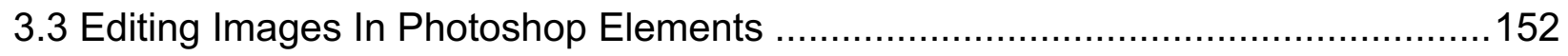

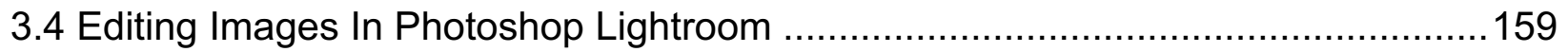

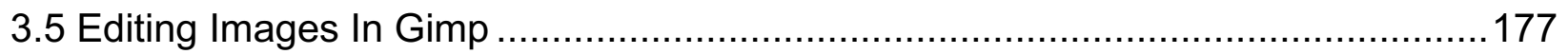

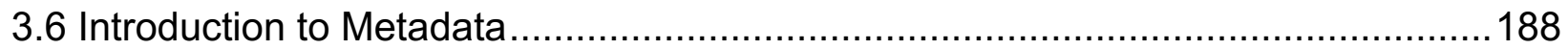

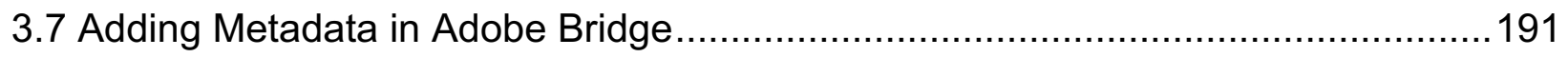

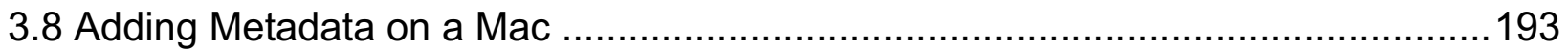

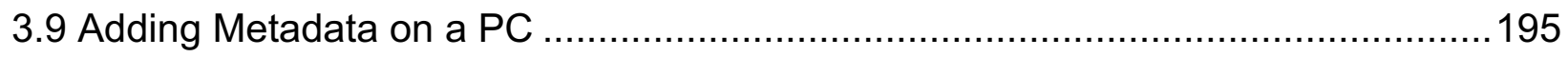

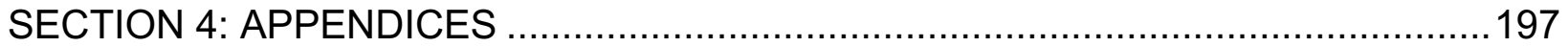

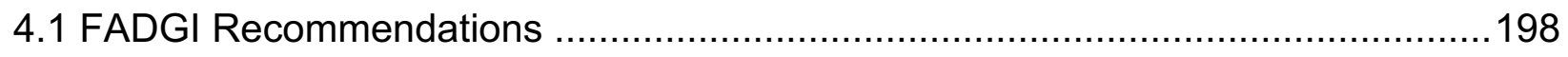




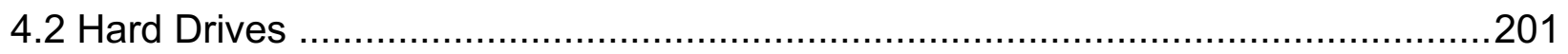

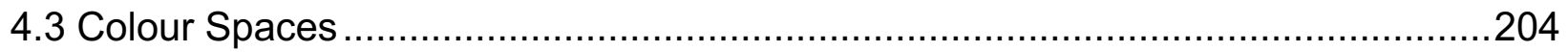

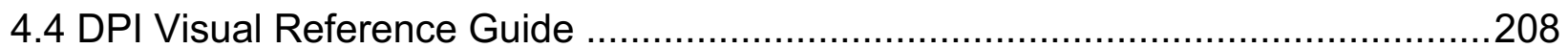

4.5 HoverCam Solo 8Plus Quality Review ...............................................210

4.6 Digital Collections and Crowdsourcing Projects ..........................................214

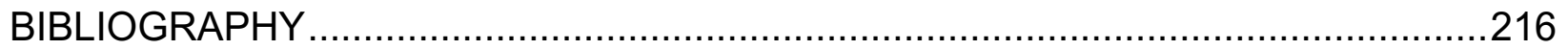

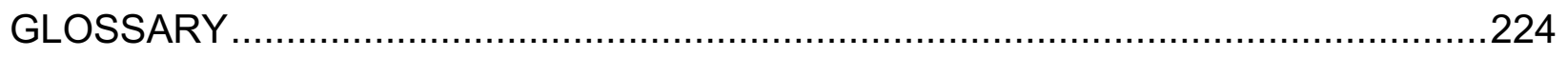

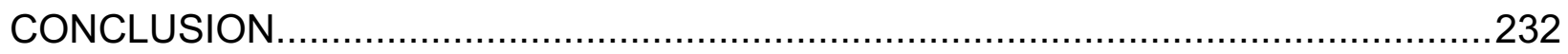




\section{INTRODUCTION}

As the technology of digitization, digital curation, and collection management advances exponentially, it is understandable that keeping up may be a difficult and daunting prospect for cultural heritage institutions. It is with this issue in mind, and with the desire to devise a solution that will be available as a handbook to organizations and individuals, that have guided the following research and approach to available literature. The following sources fall into six categories-theory, ethics, case studies, standards, budgets, and finally, digitization as it pertains to genealogists and genealogical organizations. Because digitization and digital archiving and preservation are still new additions to archival practice, much less has been written about them in comparison to other established methodologies, despite a growing demand for access to collections through digital platforms, necessitating the digitization of archival records and objects.

Most organizations and institutions are well aware of this next wave in archival practice-an effort championed on a large scale by genealogical nonprofit organizations-but one of the biggest preliminary hurdles in actively digitizing collections, even for internal and preservation purposes, is the action of digitization itself. Even with outsourcing or assignment of digitization to contract specialists or designated individuals, the challenge of determining what equipment and applications to use in order to best use an organization's small budget and limited manpower remains in the hands of staff and volunteers who may not have the expertise or training. Even fewer resources are available to non-professionals and enthusiasts who may wish to preserve, organize, and digitize their family collections. Information regarding the tools, 
methods, and step-by-step procedures needs to be more accessible to enable an individual without significant knowledge of digital imaging workflow to successfully digitize their personal archival collections.

In order to better understand the needs and experiences of the target audience for the digitization workbook-for example if such a workbook would even been needed or useful-a questionnaire was distributed via email to members of the Ontario Genealogical Society. Workbooks similar to the following are few and far between and that the lack of documentation could be due to either a lack of need for such guidance or that the need for non-professional digitization documentation has not yet been realized on a larger scale. The need for DIY digitization has definitely been identified as a lucrative market, as was made very clear during the 2017 RootsTech genealogy convention in Salt Lake City, Utah. Because it is clear that the interest is there, at least in the genealogical community, it was important to know if the knowledge and experience was there as well.

The questions were as follows:

1. Which operating systems do you use most frequently?

2. Do you have genealogical or family history documents that you would like to digitize?

3. If so, what types of materials?

4. Have you ever digitized any of these documents before?

5. If so, what method did you use?

6. In what file format did you digitize these materials?

7. Do you have a method of backing up your digital files?

8. If you do not back up your digital files, why not? 
9. Would a handbook outlining the different ways to digitize genealogical and historical family documents (and providing illustrated step-by-step instructions showing how to do so) be useful to you?

Knowing what operating system was most often used would help tailor the workbook to the commands and features unique to that system. While the underlying steps and principles are the same regardless of the operating system, attempting to follow instructions provided with a different operating system can be confusing for those unfamiliar with it. The workbook needed to be comprehensive and approachable, and presenting instructions in the most widely-used interface is a foundational way to do so.

A total of 263 people responded to the survey. Of those, $81 \%$ said they used Windows operating systems, $16 \%$ used Mac, and $3 \%$ used Other. Based on these results, it was clear that instructions should be illustrated within a Windows operating system wherever possible. Surprisingly, 73\% (194 people) had digitized archival materials before, but only $28 \%$ of those 194 (5\% of the total number of people surveyed) had digitized as TIFFs, the FADGI-designated archival file type. Only $11 \%$ had used TIFFs exclusively. So of 263 people, 54 people had digitized using TIFFs, and only 6 had used TIFFs only. It is unknown if those who listed JPEGs and PDFs in addition to TIFFs had converted original TIFFs into JPEG and PDF access files (nonarchival file types), or if the original digitization file formats were JPEGs and PDFs. Regardless, it was clear that an emphasis on archival digitization standards and file types would be essential. 
While in Salt Lake City attending the RootsTech conference, organized by FamilySearch, none of the digitization and digital archiving lectures emphasized the importance of archival file types. Virtually no one scanned their materials as TIFFsopting for PDFs and JPEGs instead-and only several knew what a TIFF file was. In the conference marketplace, a massive hall filled with commercial booths and displays, many companies advertised apps designed to allow the user to take a photograph of a document, add basic tags, and share via social media. While certainly not using RAW or TIFF formats, none were aware of archival digitization standards. Several companies offered digitization services in which the client would fill a box with film, prints, or any kind of analog materials they wished to have digitized, mail it to the company, and within a couple weeks, the company would return the original materials with either a CD of digital files or a download link. When asked if they could digitized as TIFFs if requested by a client, several salespeople did not know if this was possible.

While the digitization of an individual's personal records may seem like a proverbial drop in the bucket by comparison, it is no less valuable. Organizations like Ancestry.com and FamilySearch focus heavily on existing archival collections, especially vital statistics—birth, marriage, and death records-which are certainly valuable tools for tracing one's lineage back through public record, but do very little by way of creating a fuller, richer, and more detailed personal history. These details are preserved in the private archives, the photographs, letters, and ephemera that are often and understandably excluded in larger archives and museums. Unless directly tied to historical events or people, their quotidian and personal nature precludes them from inclusion in larger archives and museums. However, with the increasing access to 
digitized archival records via the internet, it is becoming easier to share these personal archives, bypassing the brick and mortar archival institutions all together. Even while these 'leviathans' create factory-style automated assembly lines for digitization, there is a vast, untapped wealth of information stored-for better or worse-in basements, attics, storage units, closets and the like. It is impossible and impractical for all of these things to find their way into museums and archives, but with the growing ubiquity of digital applications and digitization equipment, these records can be accessed and shared in digital form.

The experiences at RootsTech, survey responses, and research conducted, illustrate a clear discrepancy between standards of the cultural heritage community and that of the wider public. In a commercial society where emphasis is placed on convenience, interconnectivity, and mobile apps, it is not surprising that information about backing up files-let alone digitizing analog materials-has been relegated to institutional websites, often buried beneath piles of links, perhaps with the occasional sensationalized "listicle" (list-article) that makes its way around Facebook or Twitter for a day or two. If someone really wanted to know about digitization standards, it is not likely something they would stumble upon during their daily web content consumption. While this handbook does little to remedy this, hopefully someone with even a casual interest will be able to read through and gain a better understanding of the whole process. There is no downside to closing the knowledge gap between institutional archival digitization practices, what is comprehensible to and practical for the average 
person interested in preserving their family's historical documents. It is my hope that Archival Digitization for Non-Professionals will help achieve this.

My approach to this digitization manual has been a casual one, not because I don't care enough to make it formal, but because casual is not intimidating. So often when I talk to people who are interested in learning how to digitized their family heirlooms, they feel intimidated and discouraged when they find themselves sifting through web page after web page, pdf after pdf, each one loaded with jargon and industry terms, and clearly written for the professional archiving community. I feel that it was important to eliminate as much technical and industry jargon as possible, and when impossible, to provide thorough explanations. My aim is to help bridge the divide between professional archival digitization and the kind of digitization one might do at home. There has long been a fundamental divide between the two and there does not need to be. Old family heirlooms stored in attics and basements will likely never find their way into museums, but they are still invaluable resources. They can help us trace our ancestors and discover pieces of a quotidian puzzle that as it is completed, reveals aspects of the past that were previously unknown to all except those who experienced them. These family heirlooms deserve to be preserved.

When brainstorming this project I casually asked my mother if she had any old photographs and heirlooms that she might send me to work on and test my digitization strategies. She mentioned some loose photographs, albums, framed things, certificates, and the like, all passed down through the decades and at the time I started this project, 
stored in my uncle's garage. I could not have anticipated the treasures she later sent me: black and white photographs, Polaroids, slides, negatives, certificates, ambrotypes, tintypes, a daguerreotype, and a handmade leather and paper college yearbook from the 1920 s with some of the most beautiful photographs I have ever seen. I have always loved photography and history, and studied it for years. Even so, I never expected my own family to have amassed such a wonderful collection of items over the years, a veritable treasure trove to a photo-history nerd like myself. I have been so used to seeing items like these in museums and archives, behind glass or securely stored on shelves or in draws, that to carefully unwrap a tiny gem tintype album from the late 1800 s and know that the individuals pictured within were my ancestors, was absolutely enthralling.

I have learned so much about my family while working on this manual. I learned that my relatives from New York state fought for the Union Army in the Civil War, and that my great-great-great aunt was an aspiring artist. I learned that my great-great-great uncle owned the last blacksmith shop in Rome, New York as automobiles claimed the roads from horse-drawn carriages. I learned that my great-great uncle owned an art framing business in Boston, Massachusetts, and while turning the pages of a delicate album created by my grandparents in the late 1940s, I learned that my grandfather had joined the Army Core of Engineers, was stationed in Japan between 1944 and 1946, helped create new sanitary infrastructure to combat malaria outbreaks there. He then taught civil engineering at both UC Berkeley in California and Harvard University in Massachusetts. Discovering the history of my family and piece together my family tree 
and the life events of those in its branches has held a mirror to my own life and shown me fascinating glimpses of the past.

I would like the reader to think of this manual as a cookbook. There are recipes to follow and suggested techniques and tools to try. Gather the ingredients, follow the steps, and see how the finished product turns out. Archival digitization is a learning process and it is my hope that by the end of this manual, the reader will have a better understanding of the different aspects of digitization and will feel confident in undertaking their own digitization projects. I suggest reading through the entire manual to gain a thorough understanding of the tools, techniques, and standards used before beginning a project. Things covered in depth in one section may also be applicable and useful in others. 


\section{LITERATURE REVIEW}

In approaching research of digitization, digital curation, and digital preservation methods, it is best to lay a foundation of theory, history, and ethical conversation regarding these topics. Andrea Sartori is an Italian academic who holds a PhD in Local Development from the University of Florence. He has written extensively on technology in regard to academic institutions, focusing on digitization of cultural heritage, access to digital archives, museums and social media, the history of digitization, and the role of digital technology in archives and museums, and cultural economics. In his paper "Towards an Intellectual History of Digitization: Myths, Dystopias, and Discursive Shifts in Museum Computing"1, Sartori explores the shift from analog methods of archival organization to a digitized one, and how this change, and the end product has affected the role of physical materials within archives, and of institutions as repositories of knowledge in the public consciousness at large.

As digitization and digital access to museum collections and information becomes universal, it is easy to overlook the mechanisms of and reasons behind the shift from analog to digital record keeping, as well as its effects on access to knowledge and attitudes towards cultural heritage institutions. This shift, seen as a reconceptualization, "converts from a mere homologue of the 'real' into a third 'knowledge space'-also defined as 'virtuality' $[\ldots]$ - where interpretations and experiences are generated and shared." Sartori names this 'homologue' of the real in reference to the creation of digital records that directly reflected the structure and authoritative voice created within the

1. Andrea Sartori, "Towards an Intellectual History of Digitization: Myths, Dystopias, and Discursive Shifts in Museum Computing," Digital Scholarship in the Humanities 31, no. 2 (2016): 428-40, doi:10.1093/llc/fqv006.

2. Sartori., "Towards an Intellectual History of Digitization," 435. 
physical institution, which then morphs into a "knowledge space" in which the relationship between archival objects is more fluid and mobile. This reconceptualization and "morphing" is unintentional and a result of the changing ways in which information is consumed at a wider cultural level. Swiping, linking, tagging, and sharing are new ways of interacting with information, and museums have been adopting these social media communication tools in order to reach a wider audience. In order to facilitate the practical digitization of materials in archives and museums it is important to be aware of their theoretical background, and the original historical frameworks and theories of organization and knowledge exchange around which they were originally conceived.

Understanding the intricacies of the philosophical, theoretical, and practical concerns regarding archival materials ensures that the instructions and guidance provided in the following manual will take these issues into consideration and convey them to the reader in a way that is understandable and relatable. The goal is to help readers digitize their materials in an archival manner, that is, in ways that conform to institutional standards. While the average person may not have the technical and academic expertise of a professional, they should still be able to digitize and digitally preserve their personal heirlooms.

Sherri Berger, production manager at the California Digital Library in Oakland, California, discusses how ethics are defined within archives, and how that definition has changed and become complicated with the adoption and ubiquity of digital technology, 
both in preservation and record-creation (born-digital) ${ }^{3}$. Preservation ethics cannot be given one single definition because the implementation standards vary from field to field. Berger states: "Ultimately, the ethics of preservation concerns why we must save the cultural record, who does so, what is selected, and how it is done." 4 Libraries and archives have an inherent responsibility to provide access to materials, and in order to continue to do so, these materials must be preserved. Berger believes that this is a moral and ethical responsibility. Additionally, because digital material is proliferating at an exponential rate, and more photographs being taken today than ever before, particular emphasis must be placed on the 'what' and the 'how': what records are selected and how are they preserved. However, these decisions are not easily made.

As with analog materials, deciding what to preserve must be gauged by the current significance of the object, and an evaluation of its potential future usefulness to researchers, decisions which become even trickier with digital records due to their vast quantities and the speed with which they are produced. Berger goes on to describe other hurdles unique to digital records, such as their mutability (they can be interacted with, changed, linked to other records, etc.), their fragility (digital files and hardware are not as robust as we tend to assume), and a 'need for active intervention', that is to continuously monitor the health of digital files and ensure that the file format is still accessible and readable. Berger's balance between theory and practice is useful in clearly and successfully conveying her ideas regarding digitization and ethics, and she posed a number of interesting questions such as preventing the obsolescence of file

3. Sherri Berger, "The Evolving Ethics of Preservation: Redefining Practices and Responsibilities in the 21st Century," The Serials Librarian, vol. 57, no. 1-2 (July 20, 2009): 57-68, doi:10.1080/03615260802669086.

4. Ibid., 58. 
types and programs, for example, which is an essential component of digital preservation.

Rafael Capurro, an Uruguayan philosopher of Information Ethics, approaches the topic of digitization and ethics from a philosophical angle. Capurro has published numerous papers on information technology, digital ethics, information privacy, and other topics concerning contemporary society, and information in the digital age. While not specifically about archival digitization or imaging, Capurro's paper, "Digitization as an Ethical Challenge", provides an intriguing philosophical perspective on the advantages, disadvantages, nuances, and dangers of existing in a society saturated in digital technology. He believes that digital technology marks a dramatic shift in the paradigms of systems of class, social agency, and personal privacy. Researched in conjunction with the history of technology in museums as presented by Andrea Sartori, Capurro's ideas give an important perspective into potential pushback from older generations of "archival traditionalists" 5 wo may be wary of the new Pandora's Box that digitization presents, as well as corral or ground utopian visions of universal information access with discussions of copyright, privacy, and ownership.

Case studies allow the application of these ethical and philosophical viewpoints and to reports of hands-on quantitative digitization and project planning done in specific institutions. Craig Harkema, the digital projects librarian and Cheryl Avery, an archivist, both at the University of Saskatchewan, have completed a case study of the University's

5. Rafael Capurro, "Digitization as an Ethical Challenge," Al \& SOCIETY, vol. 32 (2017): 277-83, doi:10.1007/s00146-016-0686-z. 
acquisition and processing of the collection of the late photographer, Courtney Milne ${ }^{6}$. The donation consisted of 486,000 colour slides, 54,004 raw files, and others in addition to 50,945 images on $207 \mathrm{CDs}$. In their paper, Harkema and Avery review the details of the donation, the intellectual and physical control of the material, their digitization process and rationale, technical decisions, and issues encountered. While the details of the case study are fairly normal—other than the large volume of material-this case study illustrates what other individuals and institutions have done to digitize their collections as well as specific needs, issues, and concerns. Compiling and analyzing this kind of information helps to ensure that the following proposed methods and suggestions for proper digitization are relevant, realistic, and applicable.

Written by three graduate students in the Advanced Preservation program at University of Michigan School of Information, "Project Plan for Digitization: A Structural Manual for Policy Development at the Greater West Bloomfield Historical Society," is an assessment of the historical society and the students' recommendations for the society's desired digitization initiative. It is apparent from their description of the GWBHS that the historical society is inadequate in nearly every aspect of its institution: inadequate building maintenance, no care and handling policies, and no consistent cataloguing schema. While a somewhat extreme example of a lack of institutional organization, this source was extremely useful because it outlines every step in developing a digitization and organizational scheme. The authors discussed preparing

6. Craig Harkema and Cheryl Avery, "Milne En Masse: A Case Study in Digitizing Large Image Collections," New Review of Academic Librarianship, vol. 21 (2015): 249-55, doi:10.1080/13614533.2015.1034806.

7. Jeff Nash, Sara Sterkenburg, and Paul Wentzell, 2011, "Project Plan for Digitization: A Structural Manual for Policy Development at the Greater West Bloomfield Historical Society." Written as a partial requirement of SI 644: Advanced Preservation. University of Michigan School of Information. https://www.gwbhs.org/documents/2012/11/si-644-digitization-project-plan.pdf (Accessed October 14, 2017). 
for digitization (labour, equipment, training, budget, timeline, etc.), technical standards and quality assurance (hardware, software, image capturing parameters, metadata), and digital object management (back-ups, storage, metadata migration).

Technical guidelines and standards to which all practical assessments and applications of digitization strategies must adhere to meet archival standards is another vital area of research to consider. Over the past couple decades, a variety of technical guidelines have been released by government institutions around the world, reflecting the advancing technology and consequential evolution in archival standards. "Technical Guidelines for Digitizing Archival Materials for Electronic Access: Creation of Production Master Files," ${ }^{8}$ produced by the United States National Archives and Records Administration (NARA) in 2010, is an update to one produced in 2004. It covers metadata, digital file types, digitization environments, types of scanners and digital cameras, image workflow, post-production, image processing, colour management, oversized and nonstandard material, storage and quality control. These guides allow for the familiarization and surveying of archival digital imaging standards-such as those created by the Getty Research Institute, a privately-funded institution and the Federal Agency Digitization Guidelines Initiative (FADGI), an American initiative leading the country's federal digitization research—and also to track the changes in standards over time, as well as to create projections for future changes. One of the largest problems with archival digitization, other than the sheer magnitude of materials needing to be

8. Steven Puglia, Jeffrey Reed, and Erin Rhodes. "Technical Guidelines for Digitizing Archival Materials for Electronic Access : Creation of Production Master Files." U.S. National Archives and Records Administration, Federal Agencies Digitization Initiative Still Image Working Group, 2010, 1-87. http://www.digitizationguidelines.gov/guidelines/FADGI_Still_Image-Tech_Guidelines_2010-08-24.pdf (Accessed October 14, 2017). 
digitized, is that the technology-and thus standards and recommendations-changes rapidly. It is important that the information reflected in the following manual stays relevant and accurate for as long as possible. While it is difficult to know what digitization strategies, file types, and digital archiving methods may develop in the future, guidelines developed by large institutions in conjunction with trends in digital imaging serve as a reference from which to give recommendations about best practices and procedures, as well as hardware and software. For example, FADGI explicitly states that TIFF files are the archival standard for digital image files. But while FADGI does not recommend that CDs and DVDs be used as physical storage devices, they do not make any recommendations as to the types of hard drives to use or backup strategies, so it is helpful to extrapolate from FADGl's standards of file types and longevity to include digital storage hardware.

While it is useful to have theoretical and practical knowledge of the creation and curation of digital archives, the financial factor is essential, and as the impetus for my practical thesis, required as much research as the other areas I have explored. Many companies provide digitization services, equipment, and training to institutions. However, as well-presented and thorough as they are, these services all have extremely large price tags (some ranging well into the hundreds of thousands of dollars) making them inaccessible to the vast majority of smaller institutions and individuals whose budgets are limited or virtually nonexistent.

One such imaging company, PhaseOne, manufactures high-end digital camera systems and imaging software. Because PhaseOne's target demographic is largely institutions such as the Museum of Modern Art, the Smithsonian Institute, the Getty 
Institute, their services are effective and well-conceived, but clearly well above most institutional budgets. The "Cultural Heritage Solutions Guide"9 is PhaseOne's effort to reach museum and archiving professionals in partnership with a non-profit organization called Cultural Heritage Imaging. In the brochure, they offer various types of digitization solutions (cameras, light tables, copy stands, etc.) for different kinds of collections, from digital capture and photogrammetry, to multi-spectral imaging. They suggest different product lines for different workflows and pair these with their imaging processing software. Unfortunately, because their cameras alone often retail for well over $\$ 50,000$ USD, their products are beyond what most small institutions and individuals can afford. Although the quality of capture obtained with PhaseOne's systems is undeniably incredible, it is well beyond nearly all institutional standards and realistic budgets.

Information available regarding specific budgets is limited, but this is not surprising as most institutions prefer to keep their financial matters private. However, there are reports of individuals creating similar strategies and reporting on the training, set-up, and operation costs. In "Fulfill Your Digital Preservation Goals with a Budget Studio"10, Yongli Zhou, the Digital Repositories Librarian at Colorado State University, discusses the basics of digitization and digital imaging standards and the equipment she and her team uses to digitize photographic and print material at the Colorado State University Library. She does so in a style and format that is common in internal explanatory documents and presentations discussing archival digitization, some touching on budgets and financial obligations. Most usefully, she discusses changes in

9. Digital Transitions: Division of Cultural Heritage. "Cultural Heritage Solutions Guide," 2016, https://dtdch.com/CulturalHeritage-SolutionGuide-2016-June-1.pdf (Accessed November 15, 2017).

10. Yongli Zhou, "Fulfill Your Digital Preservation Goals with a Budget Studio," March, 2016, https://ejournals.bc.edu/ojs/index.php/ital/article/viewFile/5704/8318 (Accessed October 14, 2017). 
digitization technology-movement away from scanners towards digital single-lens reflex cameras-with regards to ease-of-use, accuracy, and budgetary restrictions. While her explanations leave some questions about her decisions to choose one model of equipment over another and the practicality of some of the specific studio set-ups she suggested, her paper provides a good sense of who her target audience was (institution staff members), their prior understanding of digitization (familiar, but not expert), and the understanding that a shift in digitization practices is underway.

Information regarding the cost of equipment, applications, and services is vital to the effectiveness of the digitization workbook. Digitization equipment runs the gamut of pricing and recommending specific brands and models to fit various budget brackets is an important area to address. Many people feel that they either cannot afford to digitize their materials or do not know if the hardware they can afford is adequate. Emphasis on affordability and budgetary considerations are essential to ensuring that the widest audience possible is reached. As the overall price tag of digitization projects increases, the number of readers who will undertake their own digitization projects decreases, and this undermines the entire goal of the project: to make archival digitization easy, understandable, and affordable for non-professionals.

While working at the Ontario Genealogical Society in the winter of 2018, it became clear who the target audience of the workbook would be: genealogists, family historians, and anyone with a casual interest in those fields and a box full of old family 'stuff'. The world of genealogy is almost entirely ignored by scholars, perhaps due to its popularity, commercialization, and personal, hobbyist nature. Regardless, a growing 
public interest in genealogical research, as well as massive amounts of funding and manpower provided by organizations like Ancestry.com are both undeniable factors in the technological innovation of digitization, and indications of the future direction of digitization efforts by traditional museums and archives.

According to Nick Barratt, genealogist, genealogical consultant, and CEO of Sticks Research Agency and National Memory Bank, the sudden and dramatic increased interest in genealogy and archives is owed, in large part, to popular television shows such as Who Do You Think You Are? (BBC) and Secrets in the Attic (ITV). These shows "[examine] the past through the eyes of people who traditionally never make it into the history text books[... $]^{11}$ and make history relatable and interesting. Although they do condense what potentially took hundreds of hours of research into an easily-digestible half hour to hour-long presentation, they also showcase "[...]more historical practice on screen-actual archive research sequences and explanations of what the material under examination is, why it has survived and how it can be used to find out information[... $]^{12}$, a historian-cum-detective role that many people may not have realized was possible to experience for themselves. As more people become interested in exploring their personal histories, old family photographs, personal documents and the like are being brought down from attics, dusted off, and researched. Digitization of these personal artefacts is vital. Large organizations such as Ancestry.com and FamilySearch—“Leviathans" of genealogy, as award-winning author

11. Nick Barratt, "From Memory to Digital Record: Personal heritage and archive use in the Twentyfirst century," Records Management Journal, vol. 19, no. 1 (2009): 9. doi:10.1108/09565690910937209. 12. Ibid., 9. 
and genealogist James Beidler calls them ${ }^{13}$-are well aware of the benefits (and profitability) of such digitization and are doing so by the tens of millions of records per year. According to Beilder's article "Roots \& Branches: Genealogy 'leviathans' to collaborate on digitization", which appeared in the September $8^{\text {th }}, 2013$ issue of The Daily News, "Ancestry.com will pay $\$ 60$ million over five years to help digitize global historical records from FamilySearch's huge microfilm collection, with the expectation that about 1 billion records from around the world will become available online."14

Digitizing and sharing family heritage digitally and online is a quickly growing part of twenty-first century digital life. Articles like "Five Apps That Make Including Photos in Your Family Story a Cinch"15 by FamilySearch's Leslie Albrecht Huber, "These 3 Smart Devices Let You Record Genealogy Documents, Photos and Stories Anywhere"16, and "This May be the Fastest Way Ever to Scan Old Family Photos, and it's Free"17, both from Family History Daily (which concedes that it receives a small commission from sales of these smart devices bought through links on their website) are aimed at helping amateurs and enthusiasts tackle their own digitization projects. Applications like Forever and WeGather offer free solutions for collecting, organizing, and sharing family photographs. They even allow for the addition of metadata tagging and integrate into popular social media sites, the aim being to "encourage families to connect around and

13. James M. Beidler, "Roots \& Branches: Genealogy 'Leviathans' to Collaborate on Digitization," The Daily News, http://ezproxy.lib.ryerson.ca/login?url=https://searchproquestcom.ezproxy.lib.ryerson.ca/docview/1430783745?accountid=13631 (Accessed Feb. 15, 2018).

14. Ibid.

15. Leslie Albrecht Huber, "Five Apps That Make Including Photos in Your Family Story a Cinchm," FamilySearch Blog. May 10, 2017. https://www.familysearch.org/blog/en/apps-including-photos-familystory-cinch/ (Accessed February 19, 2018).

16. "These 3 Smart Devices Let You Record Genealogy Documents, Photos and Stories Anywhere," Family History Daily, November 30, 2017, https://familyhistorydaily.com/genealogy-help-and-howto/record-genealogy-documents-photos-anywhere/ (Accessed February 19, 2018).

17. "This May be the Fastest Way Ever to Scan Old Family Photos, and it's Free". Family History Daily, November 30, 2017, https://familyhistorydaily.com/genealogy-help-and-how-to/record-genealogydocuments-photos-anywhere/ (Accessed February 19, 2018). 
share old photographs with the basic philosophy that photographs with no information connected to them have much less value and that photographs often prick memories and get the storytelling juices flowing." ${ }^{18}$ While tools like these are valid points of entry for learning about different options available to the consumer and beginning to move one's family collection into the realm of the digital and shareable, they are only pieces of the much larger puzzle that is the digitization and digital preservation of family archives.

18. Albrecht Huber, "Five Apps." 


\section{ARCHIVAL DIGITIZATION FOR NON-PROFESSIONALS}

A Guide To Digitizing and Digitally Preserving Your Family Heirlooms

by Mia Torres 
SECTION 1: PREPARATION 


\subsection{Preparing Your Collection for Digitization}

An important part of the digitization process is ensuring that the archival materials are stored properly to ensure their longevity after digitization. As documents and artefacts age and the materials from which they are made start to break down. Proper housing and storage of the materials can help slow this process down dramatically. Archival storage provides protection from the environment in which the materials are stored, external chemicals as well as chemicals produced by the objects' deterioration, insects, rodents, and the environment in which the materials are stored.

Archival paper-based materials like envelopes and boxes are $\mathrm{pH}$-neutral, meaning they are neither acidic nor alkaline. When paper-based media ages and deteriorates, it becomes acidic. Storing these materials in containers that are $\mathrm{pH}$-neutral helps slow this process down. Archival paper-based storage is generally either buffered or unbuffered. Buffered materials are treated with calcium carbonate (an alkaline compound) to help further slow the acidification of the materials stored inside. Unbuffered containers do not have this additive. The decision to use buffered or unbuffered archival storage depends on what types of materials will be stored inside. Materials that can be stored in buffered containers include cotton, linen, jute, and other plant-based materials (so most book, newspapers, documents, etc.). Protein-based materials like silk, leather (leather-bound books, albums, objects, etc.), and parchment, as well as blueprints and albumen prints (common between 1850 and 1900 or so), should be stored in unbuffered containers. ${ }^{19}$

When handling paper or bound items, it is recommended to use clean, dry hands rather than gloves, as gloves reduce the sensitivity and dexterity of one's fingers which could in turn damage delicate materials. Use either cotton or anti-static nitrile gloves when handling photographs, small objects, or anything with a delicate surface coating.

19. "4.1 Storage Methods and Handling Practices," Northeast Document Conservation Center, https://www.nedcc.org/free-resources/preservation-leaflets/4.-storage-and-handling/4.1-storage-methodsand-handling-practices (Accessed June 3, 2018). 
Remove records from any boxes or containers that may contain bugs, mold, or dirt. If you encounter mold, immediately put the materials into 2 layers of plastic Ziploc bags. If you think you are able, only handle the material in well-ventilated spaces while wearing a respirator. Molds can be extremely hazardous and ideally should be handled by a specialist.

Remove all metal from your records (uncoated paper clips, fasteners, staples, etc.) Use a micro spatula to avoid damaging the materials.

Store negatives in polyethylene archival negative sleeves. I prefer to place negatives in archival sleeves after I have digitized them, so I only need to handle them once to avoid possible damage, rather than having to put them in sleeves, take them out, and replace them after digitizing. However, if you do not plan to digitize them right away, putting them in sleeves while you organize and asses is a great idea.

In the next section I have listed some storage materials and housing supplies you may find useful in organizing and preserving your collection 


\subsection{Storage and Supplies}

\section{BOXES:}

\section{Drop-Front Boxes}

For storage of photographic cabinet cards, cartes de visite, albums, books, etc. Come in a wide variety of dimensions.

Document Storage Boxes, Legal or Letter size

Used for storing file folders

\section{Archival Binder}

For storing sheets of negatives or prints
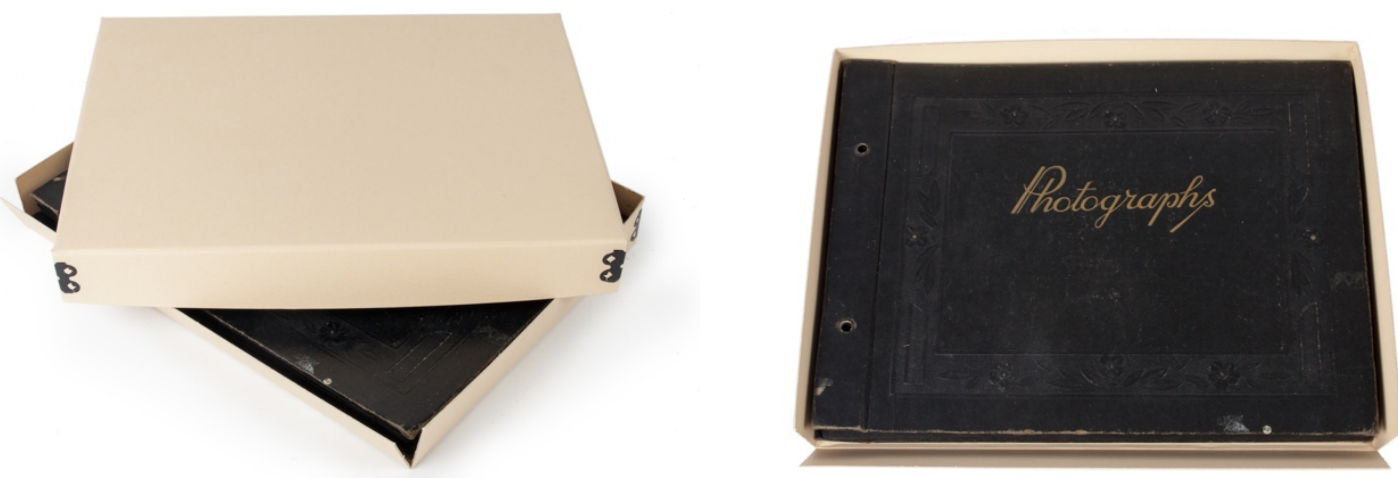

Drop-Front Archival Storage Box

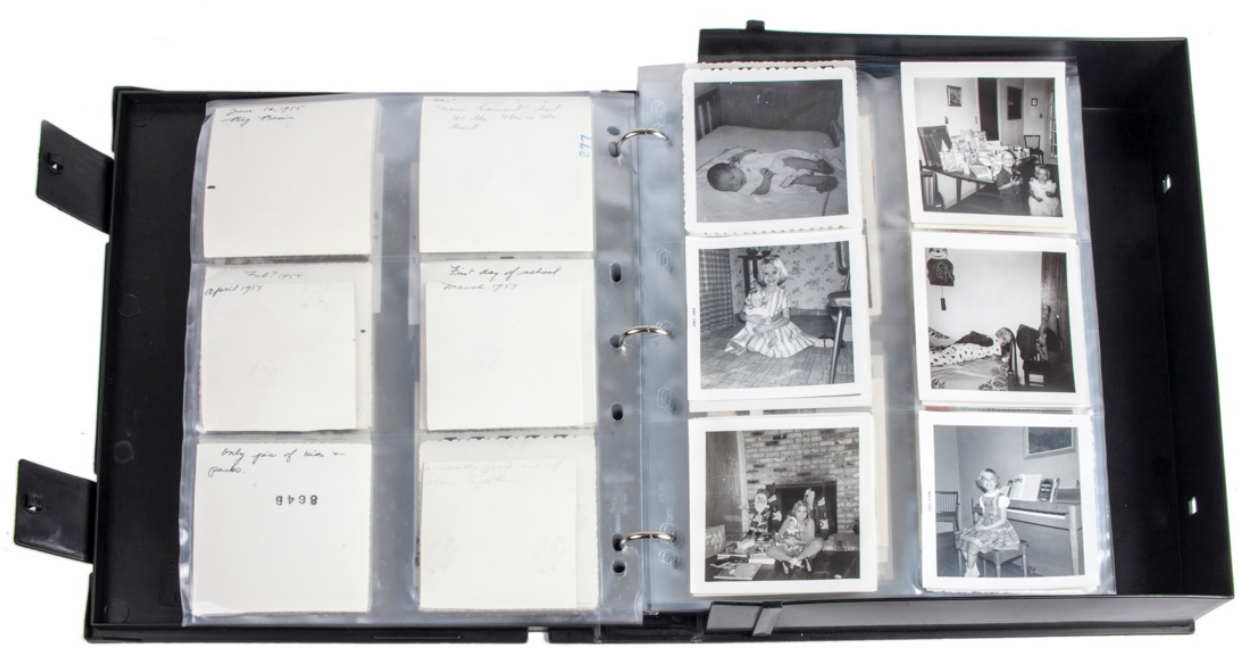

Archival Binder with print sleeves 


\section{FOLDERS:}

\section{Archival File Folders, Letter or Legal size}

Similar to common file folders, but made from acid-free archival paper stock.

\section{Map Folder Stock}

Used to make 4-flap enclosures for booklets, pamphlets, cased photographs, etc. Often used for storing large documents and maps, it can be cut down to size and used for smaller objects.

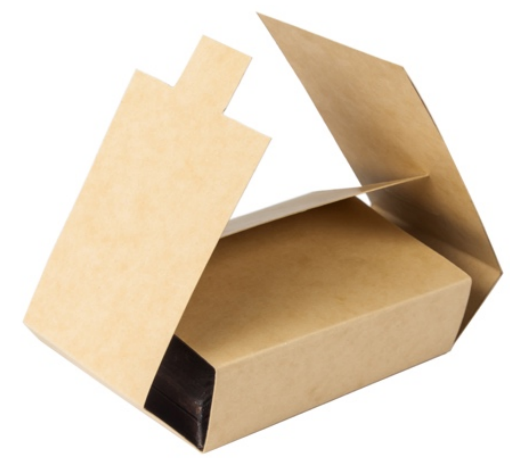

4-flap enclosure for small cased photograph

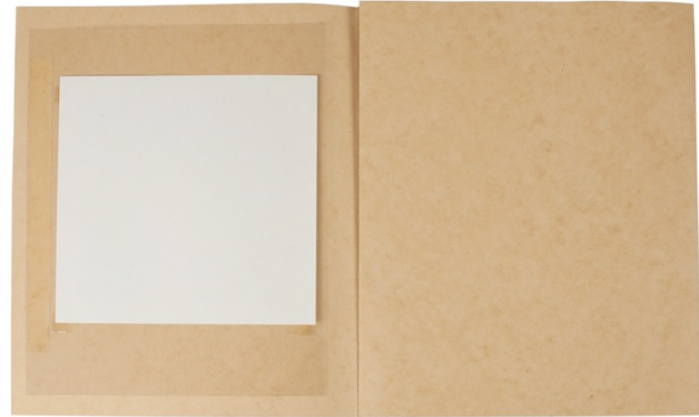

Folder with archival plastic flap taped on 2 sides with encapsulation tape

\section{PAPER \& LABELS:}

\section{Buffered envelopes, various sizes}

Should fit inside the drop front print boxes listed above, for storage of cabinet cards, cartes-de-visite, small prints, etc.
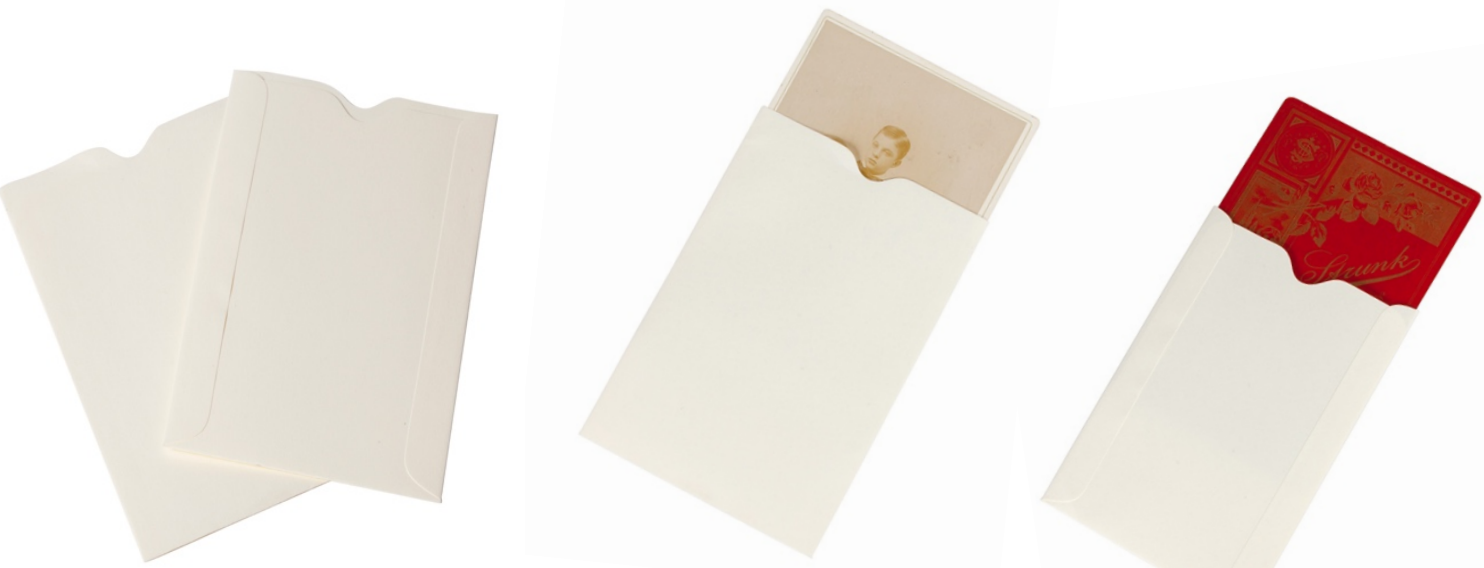

Buffered Top-loading Envelopes with Thumb Tabs 


\section{Archival Interleaving Tissue}

For use between album pages or between documents to prevent direct contact and possible dirt transfer or scratching.

Archival Labels, various sizes

Archival labels use adhesive that will not yellow and dry over time.

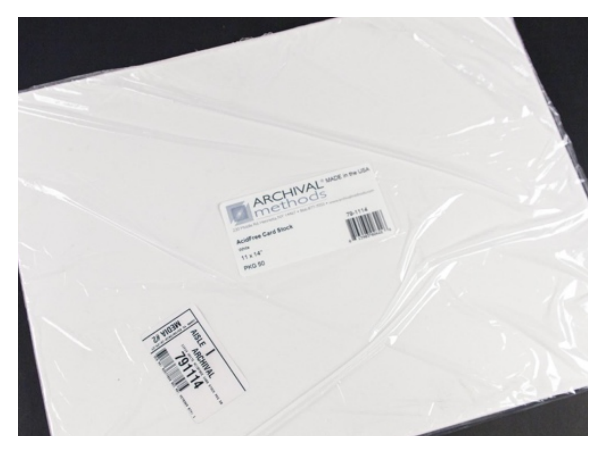

\section{ARCHIVAL PLASTIC:}

Photographs and loose documents that may need to be handled more frequently can be housed in a clear polyester encapsulation sleeve which you can either purchase or construct on your own from a larger sheet of polyester and encapsulation tape. ${ }^{20}$

Polyester Sleeve Protectors or Sheets; 3mil

Protection for photographic prints, flat delicate documents, etc. 3mil polyester can also be purchased in large rolls and cut down to size to create small encapsulations.

\section{Print File print or negative sleeves}

For storing prints or negatives either loose in a folder or in an archival binder.

TAPE:

Encapsulation Tape, 3M Tape, No. 415; 1/4 in.)

Double-sided archival tape used for making plastic encapsulations and sleeves for delicate paper-based items.

\section{Archival Linen Hinging Tape}

I prefer self-adhesive as it tends to be less messy than gummed. Used for creating hinged mat print protectors.

20. "4.1 Storage Methods and Handling Practices." Northeast Document Conservation Center. https://www.nedcc.org/free-resources/preservation-leaflets/4.-storage-and-handling/4.1-storage-methodsand-handling-practices (Accessed June 3, 2018). 
SPECIALTY TOOLS \& ACCESSORIES:

Micro Spatula (Uncoated is recommended)

Scissors

Magnifying glass

Tweezers

Pencil

Bone Folder

Ruler

Cotton or Nitrile gloves

$\mathrm{X}$-acto or Utility blade

Microfiber cloth

Coated paper clips

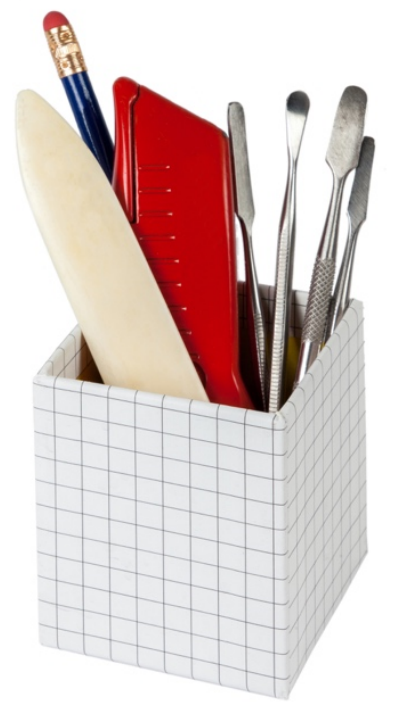

(L-R) Bone folder, utility blade, various micro spatulas

\section{SCANNING ACCESSORIES:}

Black Mat Board, large enough to cover scanner bed

It is common practice to scan documents against a matte black background to allow for clear edge definition and to enhance the clarity of thinner materials.

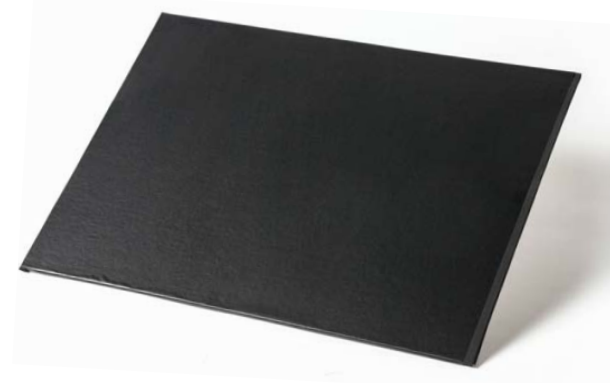

\section{Lens cleaner}

For cleaning scanner glass as needed.

Anti-static brush

For dusking off negatives and prints prior to scanning.

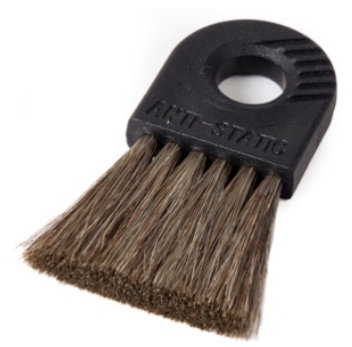

\section{SUPPLIERS}

Carr McLean (Canada)

https://www.carrmclean.ca/CategoryGroupBrowser.aspx?CategorylD=132

B\&H Photo (US, International)

https://www.bhphotovideo.com/c/browse/Storage-Materials/ci/727/N/4077634448 
Gaylord (US, International)

http://www.gaylord.com/c/Preservation

Foothills Library Solutions (Canada)

http://www.foothillslibrary.ca/category/archival

Brodart (Canada)

http://www.brodart.ca/archival-supplies/

University Products (US)

https://www.universityproducts.com/

Archival Methodsn (US, International)

https://www.archivalmethods.com/

Hollinger Metal Edge (US)

https://www.hollingermetaledge.com/\#

Global Genealogy (Canada)

http://globalgenealogy.com/archival/

\section{ADDITIONAL RESOURCES}

Northeast Document Conservation Center

https://www.nedcc.org/

National Archives

https://www.archives.gov/preservation

Sustainable Heritage Network

http://www.sustainableheritagenetwork.org/digital-heritage/category/text

Government of Canada: Museology and Conservation

https://www.canada.ca/en/services/culture/history-heritage/museologyconservation.html

\section{Library of Congress}

https://www.loc.gov/preservation/care/index.html 


\subsection{Photography Albums}

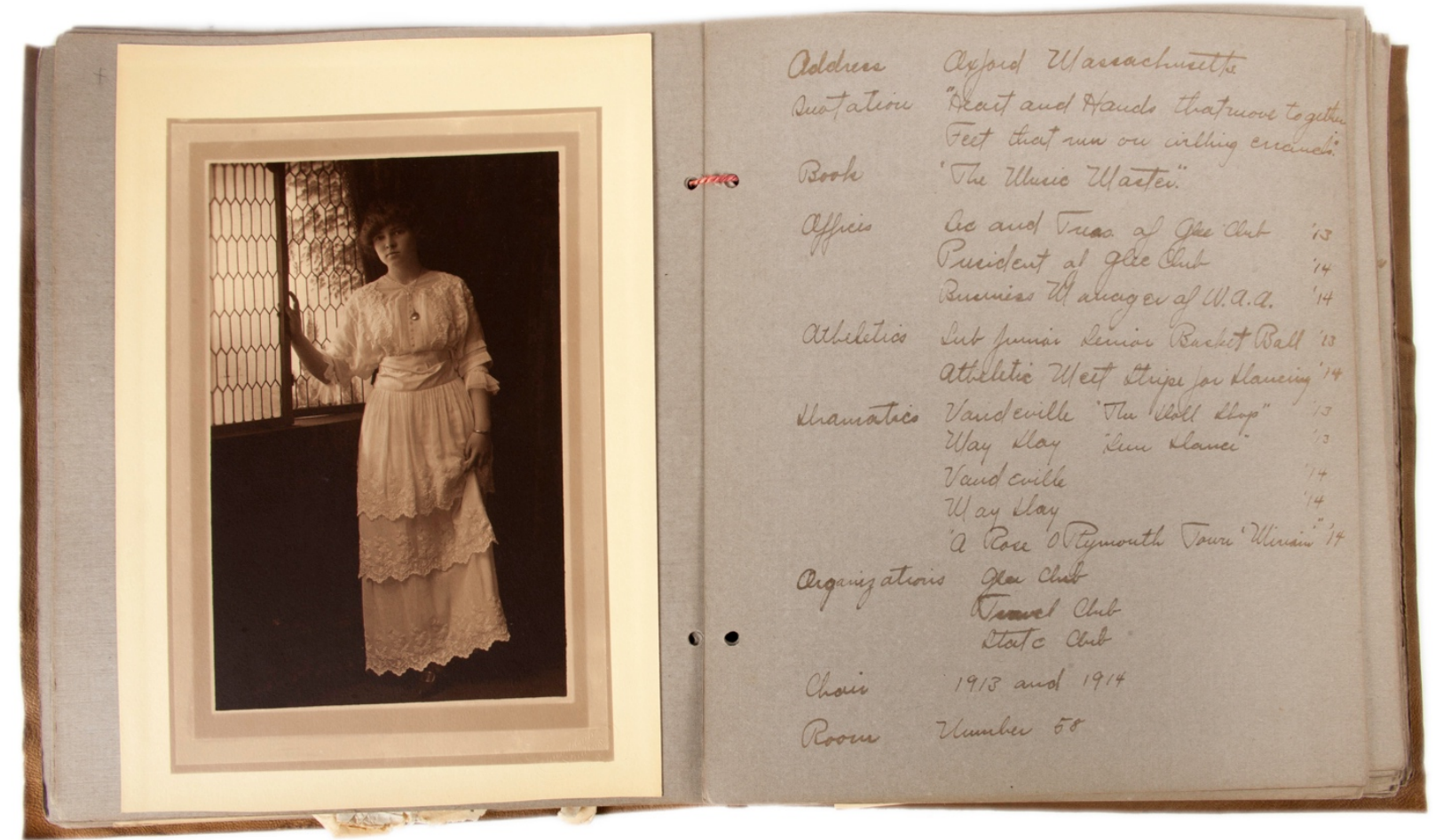

There has been some debate regarding how to digitize photographic albums. They often have multiple images on each page, and captions, notes, and ephemera included in their pages. Many archives and museums choose to remove the photographs from the pages and digitize each one individually, rather than the page in its entirety. In some cases the photographs are actually refiled throughout the archive and the original album object discarded.

Thankfully, over the past few years there has a philosophical shift in institutional approaches to arching photographic albums. Albums, especially family albums, tell the stories of the people who created them and those depicted, so consequently they are more than the sum of their parts. I am of the opinion that separating the photographseither physically or digitally_from the album as a whole detracts from, or even destroys, the overall historical and sentimental meaning of the album. 
One of the albums I am digitizing for my family is comprised of the dreaded magnetic pages, featured in Section 1.4. The thick cardstock paper coated with adhesive and covered in a thin sheet of plastic. These pages are extremely harmful to the items on the pages. Not only does the glue dry and fuse the photograph to the page, it also acidifies over time which can cause major damage to photographs. Because I want to salvage the photographs from these pages, which will inevitably continue to deteriorate and destroy the photographs over time, I am going to remove them from the album and replace them in the same position and order onto new archival pages using archival photo corners. I am going to digitize each page as it is currently, remove the photographs, scan those individually while I have them loose, then attach them to new pages and finally scan those. This may seem like a lot of extra work, but doing a thorough and complete digitization treatment of the album ensures that the photographs will not need to be physically handled again for a very long time-handling is the most common cause of physical damage to archival materials-and provides a record of the conservation and preservation performed.

My grandfather's 1945 album of his travels in Japan is another example of the different ways albums are treated as archival objects in their own right. The album consists of twenty pages with ninety-nine photographs and one newspaper clipping in total. The album is held together by a shoelace-like cord, fed through plastic posts. Some pages are double sided, some are single and some pages have titles and handwritten captions in white wax pencil—presumably written by either my grandfather or grandmother.

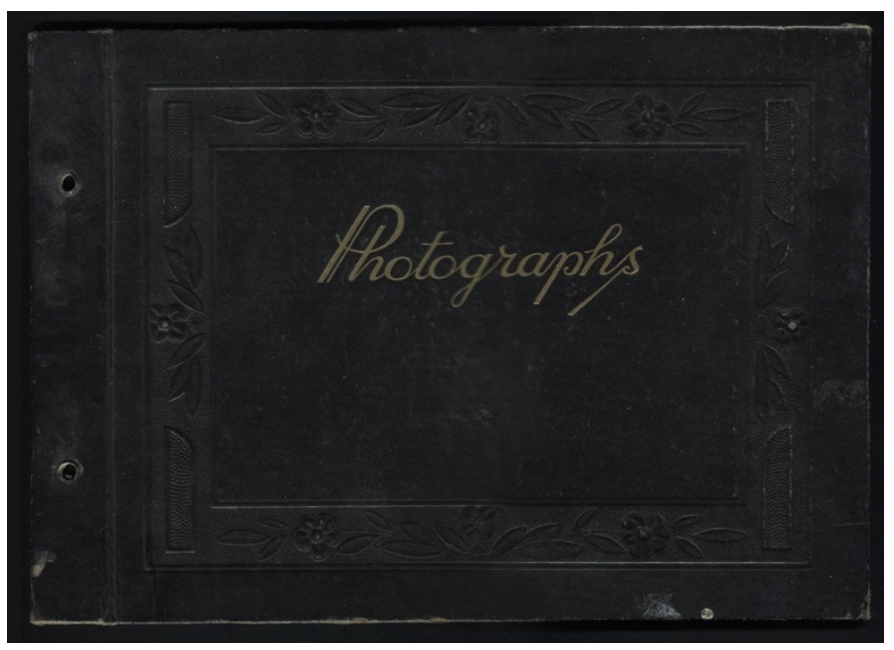



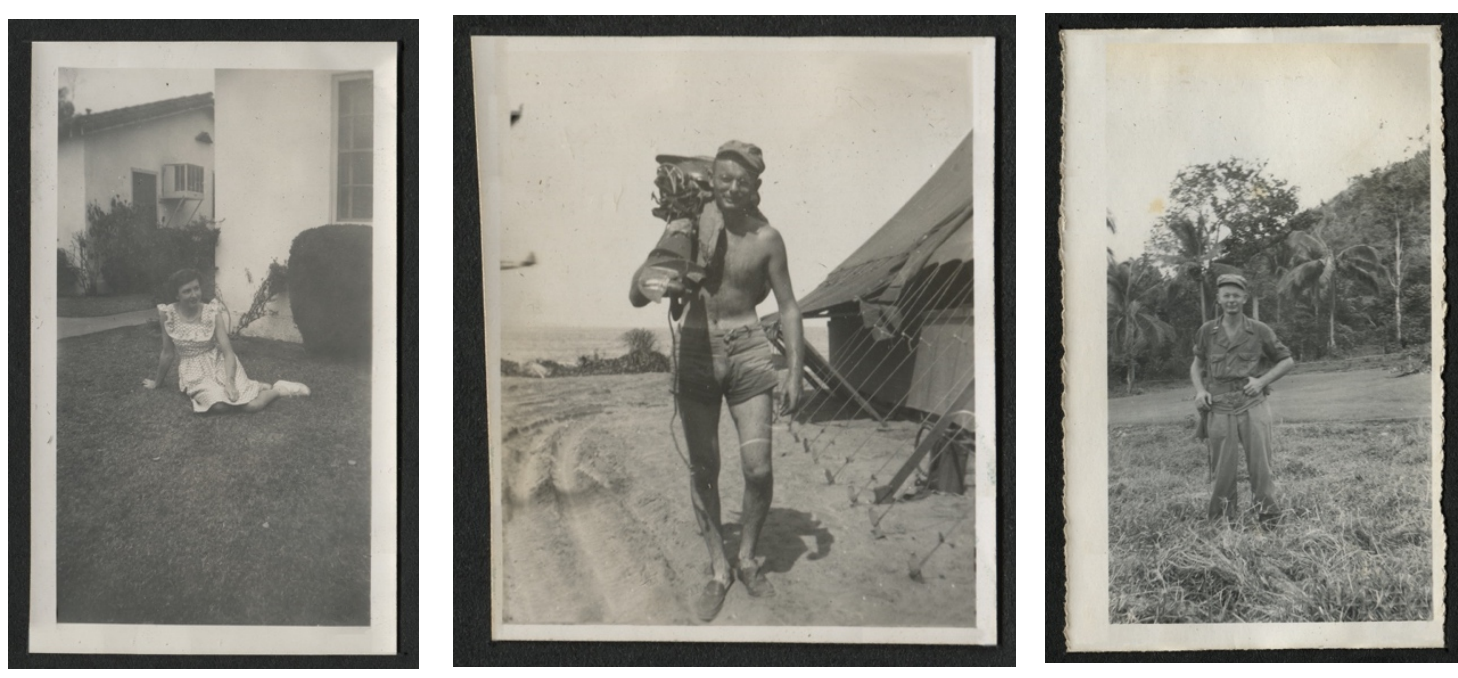

The three images above are all from my grandfather's photography album. Even with accompanying metadata documenting where the photographs came from (the album), what page they were on, their size, captions, etc. I believe you lose information when viewing them as decontextualized, stand-alone images. Below are the images in the context of the album page. You can see that the page is black, the captions are handwritten with slightly tilting lines, you can see the arrangement and relational scale, the corners used to affix the images to the page. By removing the images from their context, either physically or digitally, you lose the aura and sentiment of a 72-year old album, created by a long-deceased family member.

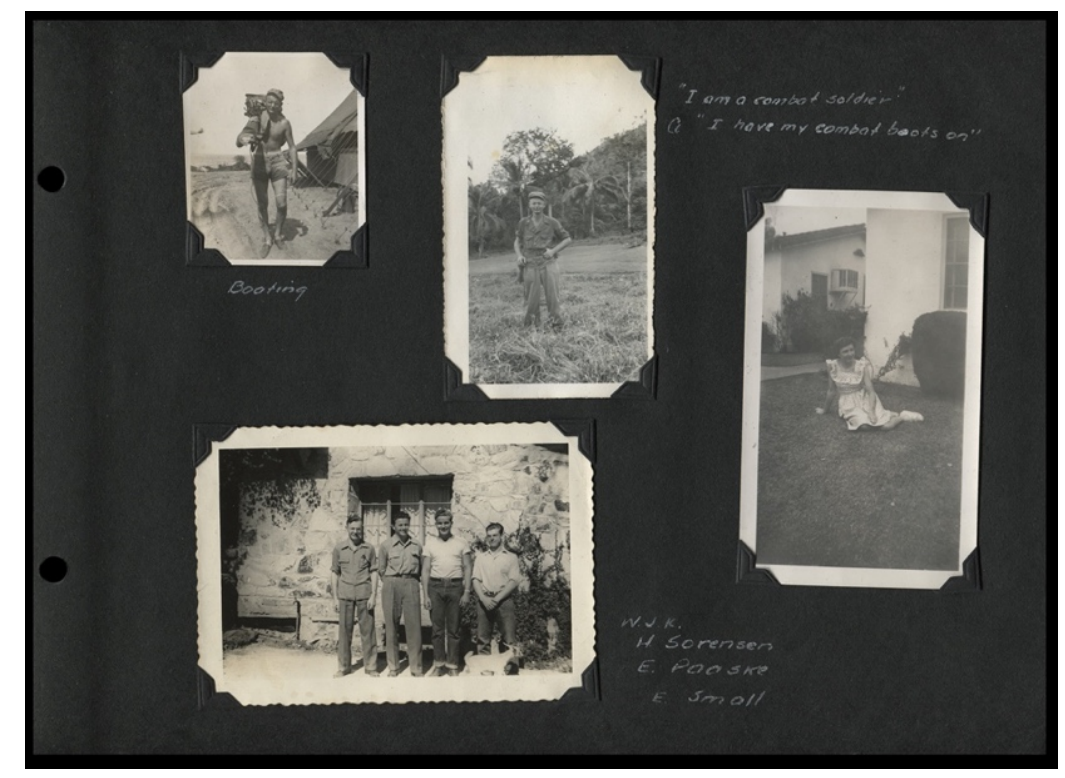

Full-page scan, pg 11, recto 
However, in instances of severe physical deterioration or contamination, removing the images from the album may be the best course of action to salvage them from the album's doomed pages. If this is the case, scan or photograph the original pages to preserve the original order and how the photographs looked on their respective pages, and then carefully remove the photographs from the album, if you are able to without damaging the photographs. Depending on the level and type of deterioration, it may be best to seek the advice or services of a professional conservator.

After I scan the album, I will insert archival interleaving tissue between each page to protect the pages from rubbing against each other, then store the album in an appropriately-sized archival box. For more information about materials and supplies, refer to section 1.3. For information about digitizing albums, refer to Section 2.

\section{ADDITIONAL RESOURCES}

Care and Identification of $19^{\text {th }}$-century Photographic Prints By James M. Reilly

Photographs Objects Histories: On the Materiality of Images Edited by Elizabeth Edwards and Janice Hart

Family Frames: Photography, Narrative and Postmemory By Marianne Hirsch

Suspended Conversations: The Afterlife of Memory in Photographic Albums By Martha Langford

Feeling Photography By Thy Phu and Elspeth $\mathrm{H}$. Brown 


\subsection{Cataloguing}

Before beginning a digitization project, it is a good idea to organize the items in a collection. This will allow you to clearly see the scope and content of the materials, as well as different strategies you will need to use to digitize, as well as potential roadblocks you may encounter.

There are different schemes for organizing archival materials but most fall into two categories: original order and intellectual order. The original order refers to the way in which the materials were organized when they were collected and compiled. For example, papers may have been arranged in a certain way by their original collector and it may be important to maintain that order as you go through the materials. Preserving the order of materials that have been arranged in a particular way by an individual can provide more information about the person and the materials than could be understood from the same materials if they were separated by type, media, date, etc. Conversely, if there seems to be no clear intentional order to a group of materials, say a box full of old photographs or land deeds it may be helpful to create a new intellectual order, one based on date, material type, persons pictured, or some other observable characteristic.

Creating an inventory of all the items or groups of things in your collection allows you to quickly view information about your collection and its scope. This can be as easy as listing the general items in a spreadsheet or as complicated as creating individual entries in a linked database using programs like FileMaker Pro, PastPerfect, Eloquent Archives, AtoM, and others depending on one's specific needs. Cataloguing is a science unto itself. But for the purposes of your home collection, a spreadsheet is a very versatile and handy way to informationally organize all of your things.

To save time, I chose to catalogue a shoebox full of my maternal grandmother's black and white photographs as a single item rather than catalog each picture as an individual item. They are all the same kind of photographs, from the mid to late 1940s, and feature 
the same group of people. There were also a handful of colour instant polaroid photographs from the late 1970s. Even though they were in the same shoebox as the black and white photos from the 1940s, I chose to catalogue them as a separate item because they were clearly a different group of photographs that had been stored together long after they had been taken.

I like to begin by laying out a number of basic criteria in an Excel or Google Sheets spreadsheet. These criteria, each being a column heading in the spreadsheet, help me organize the objects in a comprehensive layout that allows me to quickly see the scope of the collection. You can use whatever criteria seems most relevant to you and the material you are cataloguing. Below are sixteen common ones as well as descriptions of each:

1. Item Number

2. Box Number

3. Folder Number

4. Digital File Names

5. Title

6. Category

7. Subcategory

8. Document Type

9. Dimensions

10. Date

11. Description

12. Creator

13. Condition

14. Number of Items / Pages

15. Surname / Family

16. Source

17. Recommended Housing

18. Notes

\section{Item Number}

The item number is simply the number of the item within your list of items.

\section{Box Number}

When organizing the materials, you will likely be placing them in a number of boxes or containers. It is important to label these boxes, perhaps with a number or letter, and to 
record the alpha or numeric label in your spreadsheet. This ensures that instead of having to look through lots of boxes and folders to find a particular item, you can simply look at your spreadsheet to find the exact box and file number.

\section{Folder Number}

Similarly to the box number, the file number refers to a specific file within a given box. For example, Box 4 may have 10 folders in it, each folder with multiple documents in each, depending on the size and expansion capacity of the folder. Recording these numbers in your spreadsheet allows you to quickly refer to your catalogue and locate the item. The folder number may not be applicable to all items in every box, as some items may not be contained in folders due to the type, size, condition, etc. of the item.

\section{Digital File Name}

The digital file name refers to the string of alphanumeric characters you call each digital file. It can contain information such as the collection prefix, descriptive word or words, the date, and a sequence number. For more information on naming digital files, please refer to section 1.8, Creating a File-Naming Structure.

\section{Title}

The title of the item can be either a name given by its creator, such as a drawing named by the artist, or it can be a name that you give it while you're cataloguing, like 'travel photographs' or 'family Bible'.

\section{Category}

The category is the general type of object the item is, such as 'photograph', 'book', or 'cd'. The level of specificity can vary depending on the range of materials you have, but in general the categories should be broad. 


\section{Subcategory}

Identifying the subcategory of items allows you to categorize into smaller groups. For example, if you're cataloguing a box of photographs, possible subcategories you could use are 'black and white' or 'polaroid'.

\section{Document Type}

The document type is an optional category that can allow you to further specify types of objects. For example, if an item's category is 'photograph', its subcategory is 'black and white', the document type might be 'silver gelatin'. This may not be relevant or useful if the specific identification of the type or production process of your item is not a priority.

\section{Dimensions}

The dimensions are usually the length and width of an object, in either metric or imperial measurements (or both), but can also describe the format dimensions and duration of a time-based item, like a film or audio file.

\section{Date (Approximate)}

The date describes when the item was originally created, usually to the year, unless a specific day is known. In cases where you do not know the date, you can approximate within 5 or 10 years depending on the age of the item (older items often have broader date approximations). ${ }^{21}$

21. For example, I am able to determine when a photograph of my mother blowing out five candles on a birthday cake would have been taken because I know her birth date and just need to count forward five years. But when dating another photograph of my great-great-great aunt, Lucretia Pearson, I would list the approximate date as c. 1875, based on the type of clothes the woman is wearing and other clues in the photograph as well as the physical photograph itself. If perhaps I or someone in my family does some genealogical research in the future and discovers the birth or death date of this mysterious greatgreat-great aunt of mine, that could further help me narrow down the date of the photograph. Dating archival material is a fascinating and detailed subject of its own and if you're interested in learning more, I highly recommend venturing down that rabbit hole. 


\section{Description}

The description is a brief string of phrases or a sentence or two describing the item(s). It can describe both the subject matter of the item as well as it is easily identifiable attributes.

\section{Creator}

The creator is the person who originally created the object-the photographer, author, artist, manufacturer, etc. You may not know the creator of your items, and that is ok.

\section{Condition}

Condition refers to the physical status of the item in terms of any kind of wear or damage. This could be anything from creases and tears to mold and staining.

\section{Number of Pages / Items}

The number of items or pages refers to how many individual objects 'belong' to each catalogued line item. For example, item \#1 in my Kaufman Family Archive spreadsheet is a group of 37 black and white photographs. Item \#4, a cased Daguerreotype image is 1 item, while Item \#9, my mom's Peru Travel album, has 4 pages (8 sides).

\section{Surname / Family}

If you are a genealogist or genealogy enthusiast, you may want to keep track of which branch of your family tree each item in your collection belongs to. Including a 'Surname' or 'Family' section could be useful to you.

\section{Source}

If you find yourself becoming the repository (or perhaps dumping ground) for your family's heirlooms, you may want to keep track of where everything came from and who had what. This could help you in the future when trying to piece together the history of your items by tracing their provenance. 


\section{Recommended Housing}

Making a note the recommended housing for an item or group of items can help you get an idea of the types and amounts of materials you will need to properly store the items after they have been digitized.

\section{Notes}

Notes is a catch-all field for any pertinent information or comments you would like to include in your spreadsheet.

Following the above criteria, I created an inventory spreadsheet for my family's things. Here are the first ten items (see an enlarged version at the end of this section):

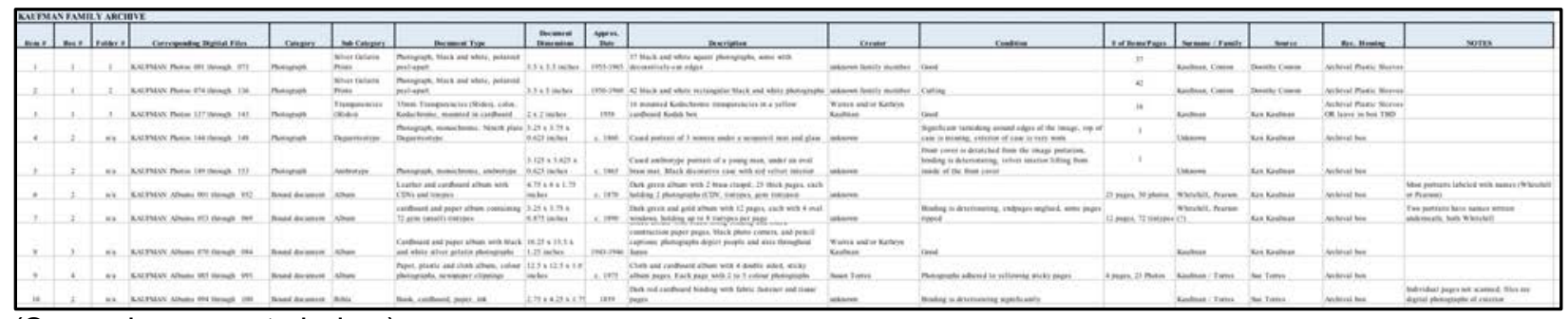

(See enlargements below)

It might be helpful to create what in the world of archiving is known as a 'controlled vocabulary', an established set of terms used for things like names, descriptions, materials, and so on. For example, while going through your things, create a list of general materials-photographs, letters, certificates, legal documents, recipe cards. You may then want to have sub categories for each. Under photographs, you might want to include colour photographs and black and white photographs, cased photographs. Under 'books' you may have Bibles, yearbooks, baby books, diaries, etc. It all depends on how many things you have, the variety within that group of things, and how specific you want to be (which depends on how much time you want to dedicate to the project).

It is important to remember that there is no "wrong" organizational method, as long as it is consistent, does not harm the items, and makes sense to you and anyone who may 
be accessing the material. Institutions and large archives have their own established "right" way to organize things, often based on suggested national guidelines, established tradition, and convention, but when it comes to your archival material, it is important that you create a system that works for you.

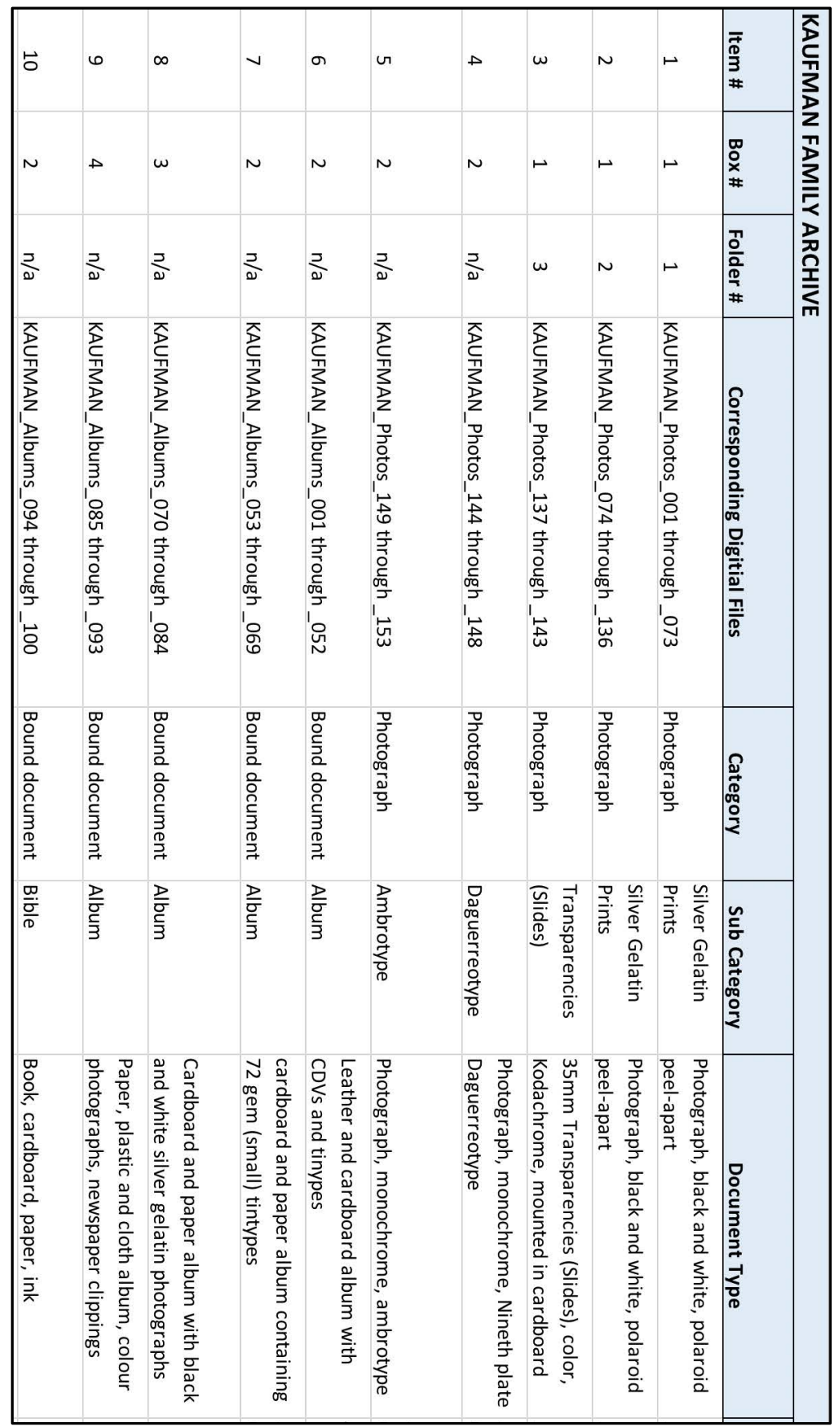




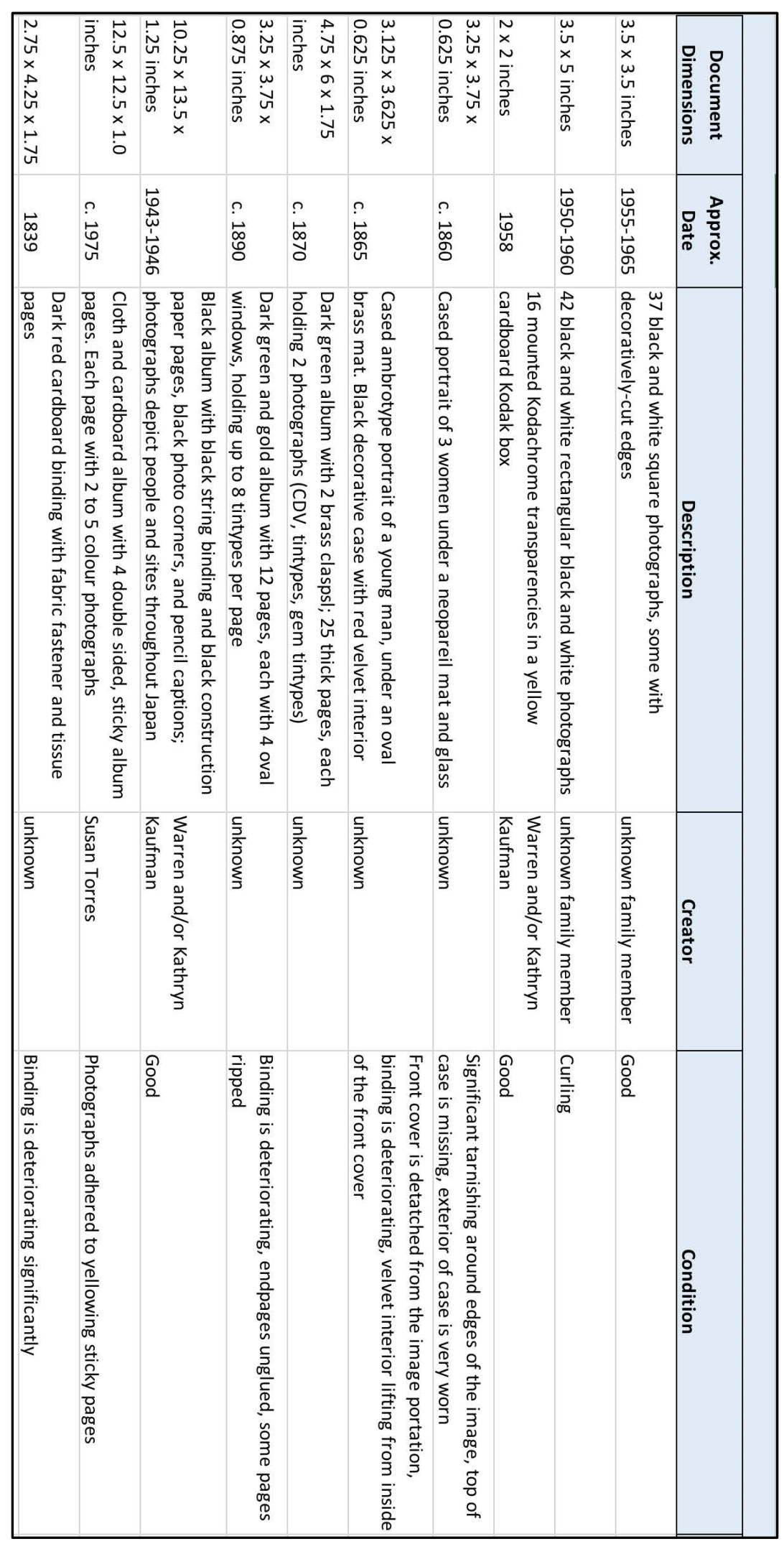




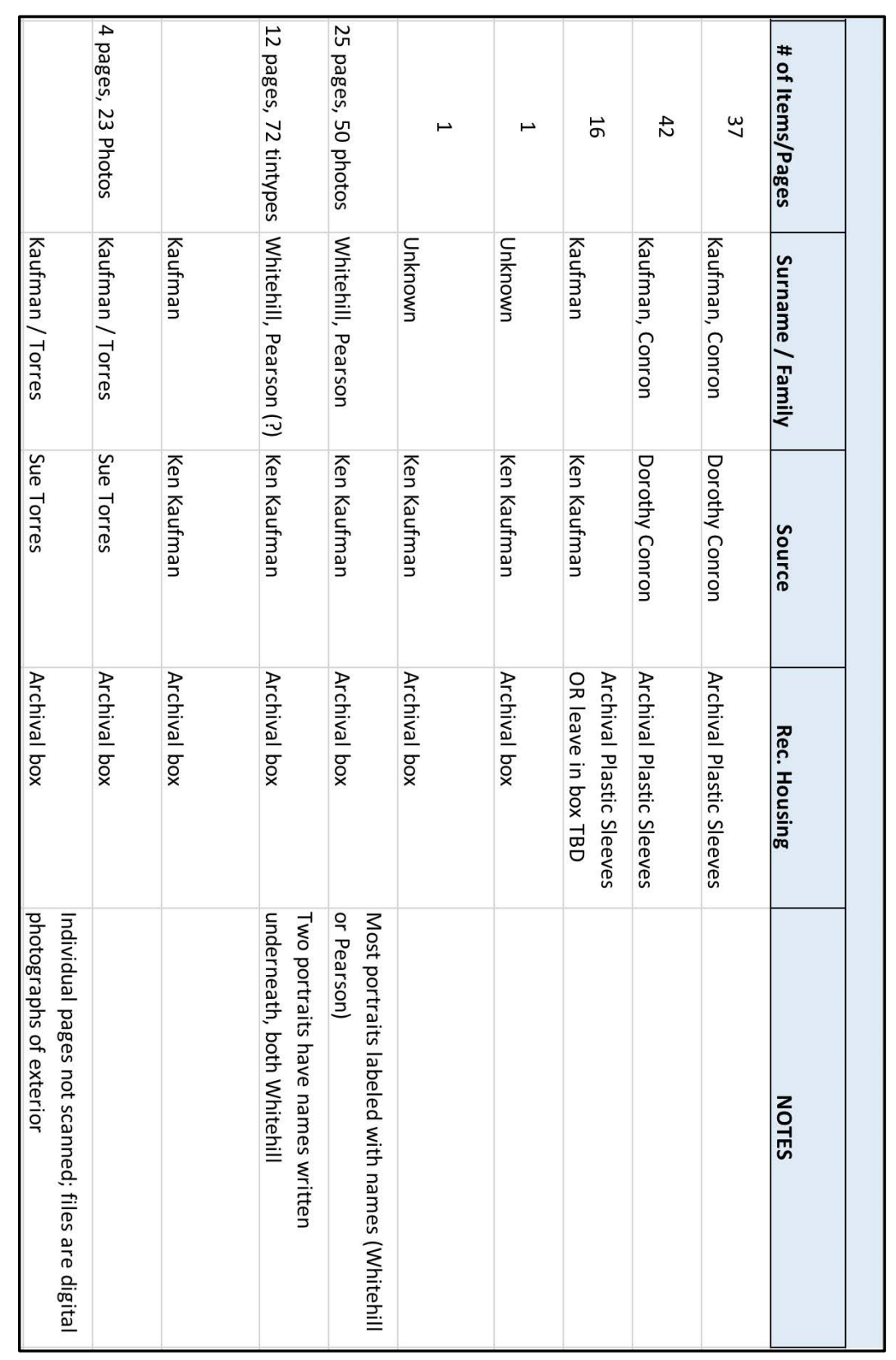




\section{ADDITIONAL RESOURCES}

1. Cataloguing Cultural Objects: A Guide to Describing Cultural Works and Their Images by Baca Murtha

2. "Cataloguing Archives In Four Easy Steps" ay the British Postal Museum https://postalheritage.wordpress.com/2013/09/25/cataloguing-archives-in-four-veryeasy-steps/

3. "How Do Archivists Organize Collections?" by Samantha Thompson, Archivist at the Peel Art Gallery Museum \& Archives

https://peelarchivesblog.com/2015/08/26/how-do-archivists-organize-collections/

4. "The Small Museums Cataloguing Manual: A Guide to Cataloguing Object and Image Collections" edited by Hilary Ericksen and Ingrid Unger for Museums Australia (Victoria) https://mavic.asn.au/assets/Small Museums Cataloguing Manual 4th.pdf 


\subsection{Derivative Files and File Types}

One of the questions I am asked most frequently is: What file type is best for scanning? The follow up question is usually: Why shouldn't I scan as a Jpeg or PDF? The short answer to this question is that you should be scanning each item as a TIFF, because it is an archival file format. The long answer is much more complicated and nuanced, but I will attempt to provide some general answers so you can make educated decisions about what file types to use in your future digitization projects.

An important thing to remember is that any digital copy is better than no digital copy. If you realize that you have scanned lots of things as Jpegs or PDFs, do not worry. If you still have access to the materials you digitized then you can re-scan them as Tiffs if you have the time, if not, that is ok. However, moving forward it is important to understand the differences between file types and to know why some are better for archiving than others so you can begin to (or continue to) digitize your collection wisely.

So what is an 'archival' file format? An archival file type is one that has been designed to retain all of the data with which it was originally created, unless deliberately altered by the user. They are also called lossless file types because there is no data loss. Common lossless image file types include TIFF, BMP, PNG, and RAW. ${ }^{22}$ Lossy file types on the other hand, lose data when opened and edited. This helps keep the file sizes smaller and more easily manageable by some programs. They don't lose much data each time, and it likely is not noticeable to the average viewer, but over time the loss adds up. Given the rate of technological advancement over the past thirty years or so, there's no telling the ways in which these archival files will be used and viewed in the future, as well as the longevity of their physical counterparts, so it is wise to aim for the highest standard and quality. Just like physical items in archives, it is best to take measures to ensure that the items housed within-in this case digital files-remain intact for as long as possible.

22. For more information about these file types, please refer to the glossary. 
So, what do you do if you have a virtual pile of PDFs and JPEGs, and are not able to rescan the original source material? Here are several strategies:

1. Using some programs, like Adobe Acrobat Pro DC, you can export TIFF image files from PDF documents. Say, for example, you have a 10-page PDF document of scanned letters from the 1860s, and either the original files are inaccessible or you do not have time to go back and rescan them as TIFF, for whatever reason (although, if you are able, I strongly recommend that you try to). You can open the PDF file in Acrobat, go to FILE > EXPORT TO > IMAGE > TIFF.

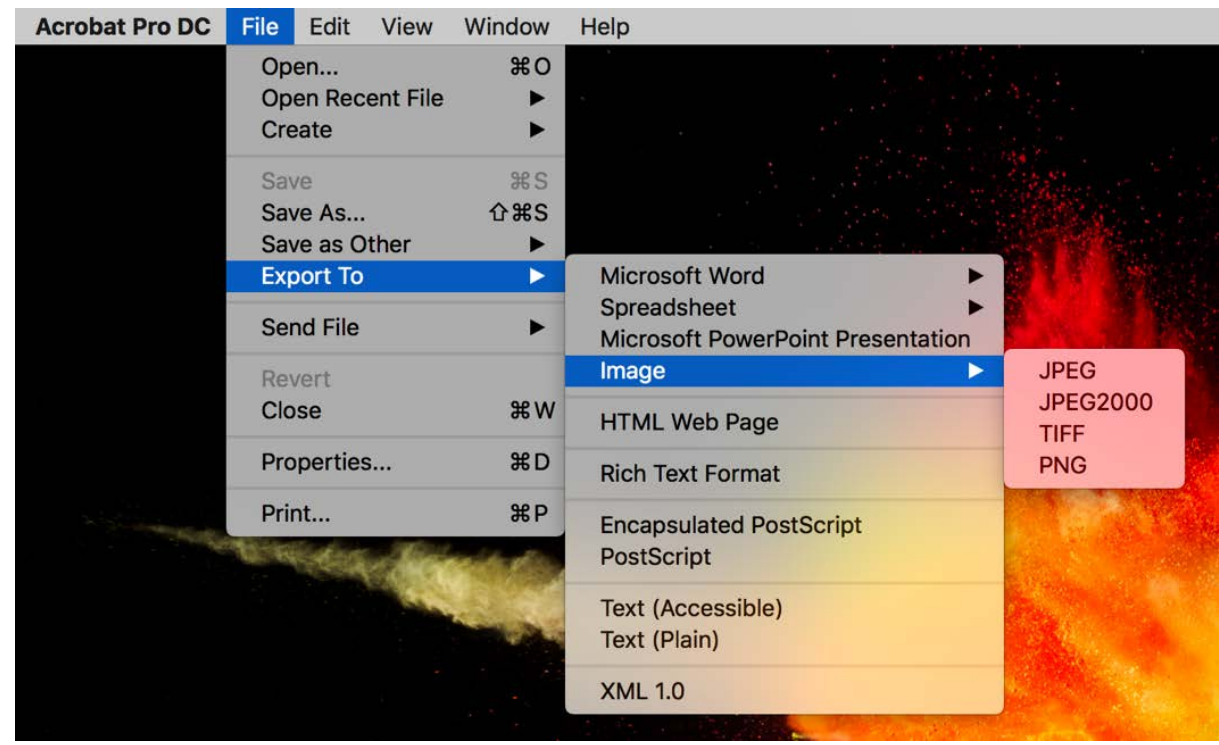

Then, select the folder where you want to save the exported TIFFs. If you have not exported TIFF images from a PDF document in Acrobat before, you will need to click on the SETTINGS... button next to the file format dropdown menu. Make sure that the file settings for monochrome, Grayscale, and colour are all set to either NONE or LZW.

Finally, click SAVE, and the images will export using the original PDF file name with the addition of Page_1, PAGE_2, etc. at the end. 
2. If you have lots of JPEG scans, I recommend creating a copy of each (which can be done as a batch command) to set aside and leave untouched as the 'master' file. This way you will have one set of JPEGs that is archived away, not to be opened or edited (the "master" files), and one copy that can be edited, copied, uploaded, etc.

You could theoretically save the files as TIFFs (import the JPEGs into Lightroom and then export them as TIFFs, for example), but this can be tricky as it involves resampling the image-essentially telling the computer to generate pixel data to expand the JPEG file to a larger TIFF file. While computer resampling algorithms are good, and getting better, they still can't compare to original data collected from a direct scan. Because of this, I would recommend only using this method for text-based documents, not for photographs or image-heavy documents in which there is a lot of colours, gradients, etc. (detailed pixel data). 


\subsection{Creating File-Naming and Folder Structures}

Naming digital files using a consistent naming structure is vital for organization, retrievability, and accessibility. Ideally, you should be able to look at any file on your hard drive or in your digital archive, and know exactly what it is, or at least what group of materials it belongs to. ${ }^{23}$

Before beginning a scanning project, assess the items you want to digitize. Are they all from the same collection, family, surname, or even box? When laying out a file naming structure, I like to start with the biggest umbrella and add suffixes for each additional sub-section. For example, when creating a file naming structure for my own family's collection of materials, I have several options. If I know that everything in Box "1" is Kaufman family items (my grandfather's surname), Box "2" contains Conron family items (my maternal grandmother's maiden name), I can begin the file names with those surnames respectively. Use underscores $\left(_{-}\right)$or dashes ( - ) to separate words or sequence numbers in a file name. Try to keep file names short (but still clear) and do not use spaces, periods, or special characters! These can make the files unreadable by some applications.

\begin{tabular}{ll} 
Box 1 & Box 2 \\
\hline KAUFMAN_ & CONRON_- \\
or & or \\
KAUF_ $_{-}$ & CON_-
\end{tabular}

However, if things are not organized by family branches, and I don't know who most of the people are, I can use one collection name prefix for all of the files. Because everything came from my Kaufman uncle's (non-archival!) garage, I will begin all the files with the prefix "KAUFMAN_."

23. Puglia, "Technical Guidelines for Digitizing Archival Materials for Electronic Access," 83-85. 
Now that a broad prefix has been determined, you can move to the next level of description. This can be any number of things, and it is important to choose something that you think will work throughout the entire collection and is understandable to you. This could be the type of object it is (photograph, letter, deed, etc.), a specific event, a subject category, etc.

$\begin{array}{ll}\text { For example: } & \text { KAUFMAN_Tintype_- } \\ & \text { KAUFMAN_Polaroid_ } \\ & \text { KAUFMAN_Slide_ } \\ \text { Or: } & \text { KAUFMAN_Photo_ } \\ & \text { KAUFMAN_Album_ } \\ & \text { KAUFMAN_Letter_ } \\ & \\ \text { Or: } & \text { KAUFMAN_Wedding_ } \\ & \text { KAUFMAN_Baby_ } \\ & \text { KAUFMAN_Travel_ }\end{array}$

Using uppercase letters is not mandatory, but I find that using uppercase for at least the prefix helps make the files quickly readable and identifiable. Just be sure that your upper or lowercase usage is consistent throughout all of your files.

I have several photographic albums in my family collection, so I can use a naming convention that indicates each file as belonging to a photographic album, identifying each album more specifically, followed by a page number. 


\section{[i:::}
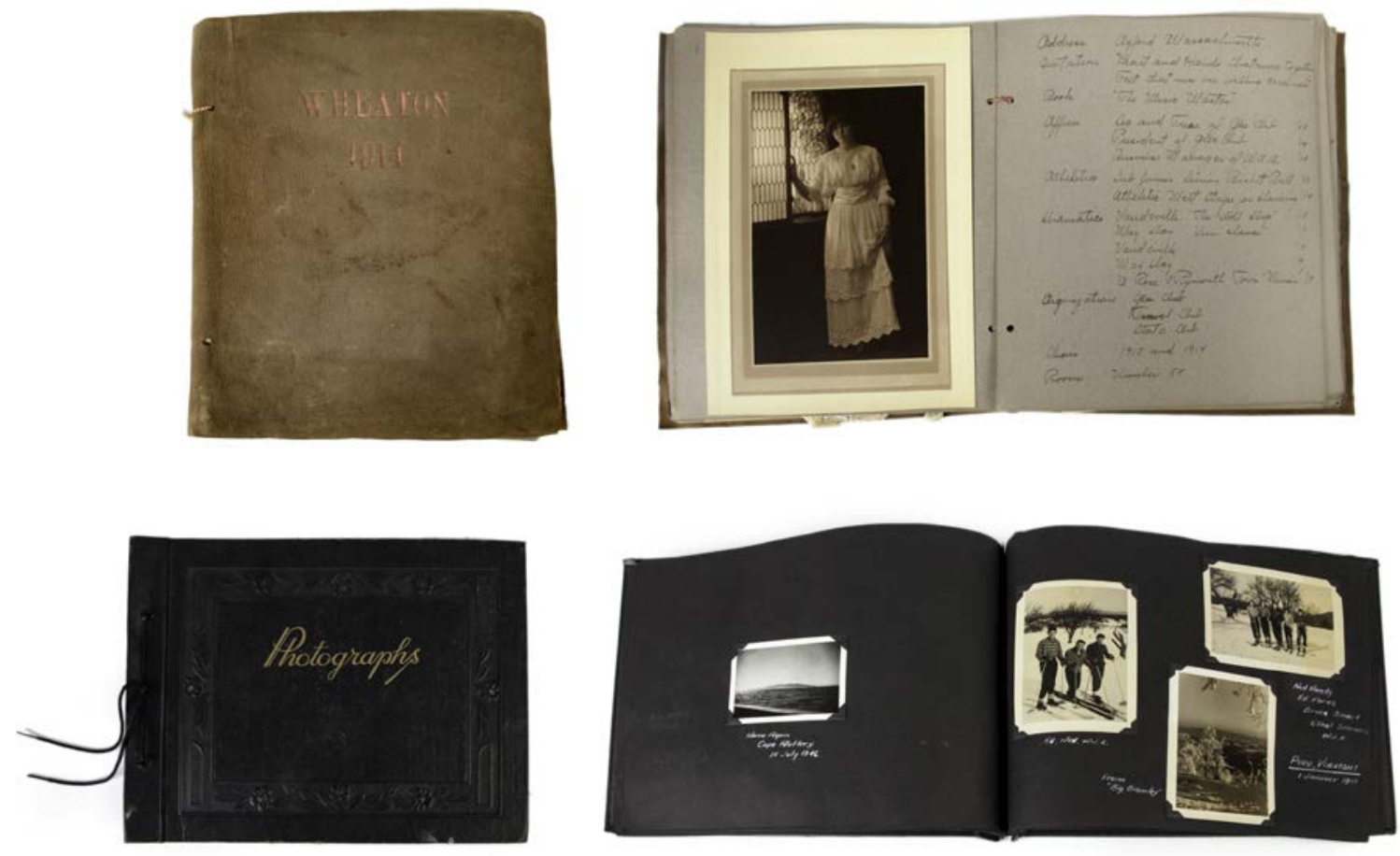

Album 1: An album of gem tintype portraits from around 1860.

Album 2: A Wheaton College yearbook from 1914.

Album 3: An album of my grandfather's travel from around 1945.

Corresponding File Names:

KAUFMAN_Album_01_pg_001

KAUFMAN_Album_01_pg_002 [etc.]

KAUFMAN_Album_02_pg_001

KAUFMAN_Album_02_pg_002 [etc.]

KAUFMAN_Album_03_pg_001

KAUFMAN_Album_03_pg_002 [etc.]
Or:

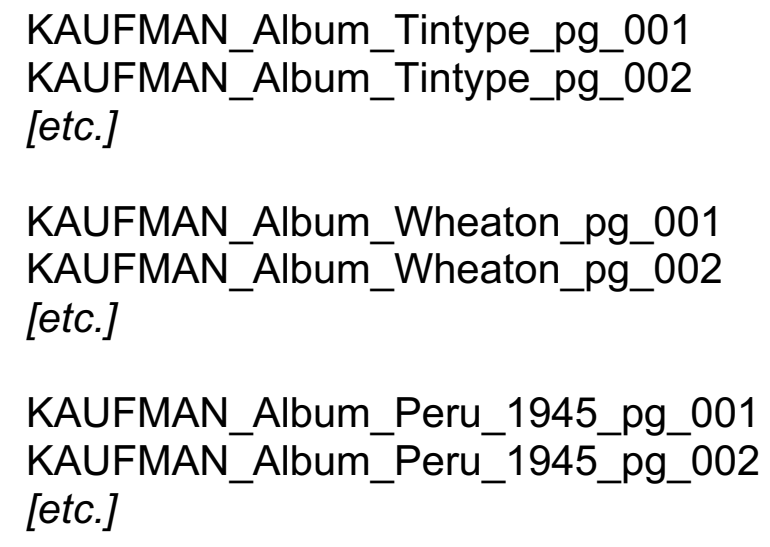


If your collection is already organized into boxes and folders, you may want to include this information in the filenames as well, or instead of a descriptive word.

\author{
KAUFMAN_B01F12_001 \\ KAUFMAN_B01F12_002 \\ KAUFMAN_B01F13_003 \\ KAUFMAN_B01F13_004 \\ KAUFMAN_B02F01_001 \\ KAUFMAN_B02F02_002
}

Whether or not you restart the file sequence number at 001 again when the box and or folder number changes is up to you, but I have found that allowing the sequence number to continue uninterrupted throughout a box is useful for quickly knowing how many items there are in that box.

Using zeros before single digit numbers is important because it ensures that the files remain in the correct order. If zeros were not used, file eleven (11) would be listed directly after file one (1), when it should come after file ten (10).

\begin{tabular}{|c|c|c|c|}
\hline \multirow[t]{5}{*}{ Incorrect: } & Filename_1 & Correct: & Filename_01 \\
\hline & Filename_11 & & Filename_02 \\
\hline & Filename_2 & & Filename_03 \\
\hline & Filename_21 & & Filename_04 \\
\hline & Filename_3 & & Filename_05 \\
\hline
\end{tabular}

When you reach double digits, do not add another zero. The total number of digits after the underscore $\left(_{-}\right)$should be consistent. If you reach 99 and realize you need a third digit, you can continue on with three digits_100. If you are comfortable using Lightroom or Bridge to rename the files to add a digit, you can do so, but it is not necessary. 
Incorrect:
Filename_08
Correct:
Filename_08
Filename_09
Filename_09
Filename_010
Filename_10
Filename_011
Filename_11

If you need to scan the back of an item as well as the front, use the same file name for both, with the edition of '-001' and '-002' (dash, not underscore) indicating the front and back respectively. If you keep this method consistent for all of your scans, you willl know that any file name ending in '-002' is a scan of the reverse side of the item. The reason for using three digits is simply because the EPSON Scan software automatically sets sequence numbers to be three digits. If you are using a different scanning software that allows you to use a custom amount of sequence digits, you can use '-1' and '-2' to indicate the front and back. I avoid using letters (like ' $\mathrm{f}$ ' and ' $b$ ') to indicate the front and back in case I need to add a different alphabetical suffix later on (like '-pm' for 'production master' or ' $v 1$ ' for 'version 1'). Numbers should be used to indicate different scans, and letters should be used to describe various copies or versions of the same scan.

For example:

KAUFMAN_B01F12_001-001
KAUFMAN_B01F12_001-002
KAUFMAN_B01F12_002
KAUFMAN_B01F12_003

The above file names tell me a lot of information. I know that the first file is part of the Kaufman collection, it is located in box 1 , folder 12 , and is a scan of the front of the document. The next file is part of the same collection and is in the same box and folder, but is a scan of the reverse of the item. I can also tell that the next two digital files, _002 and _003 are both items that only have one side scanned (because there is no -001 or 002 suffix), presumably because there is nothing on the other side. I like to add -001 to the front scan as opposed to only adding -002 to indicate the reverse side, because if I 
see a file with the suffix -001 but no file labeled -002 following it, I know that either the "$002 "$ file has been misplaced or the back of the item has not yet been scanned.

I prefer to use the numerical system (Album 01, 02, 03, etc.) rather than the descriptive method, because it is possible that I may find, for example, another album of tintypes, or another travel album in my family's collection. The file names also risk becoming too long as I add more textual descriptive sub-sections. Using the numerical series method allows for easy additions. Someone looking at the file names may not know that each album is, but they can either look at the file or refer to my cataloguing spreadsheet to view the details of that item.

Here is a breakdown of different file-naming schema:

COLLECTION_Box\#Folder\#_ItemNumber-front/back

COLLECTION_Box\#Folder\#_ItemNumber

COLLECTION_TypeOfMaterial_ItemNumber-front/back

COLLECTION_TypeOfMaterial_ItemNumber

You may notice that I have not included any file type extensions (.tiff, .jpeg, .psd, etc.). This is because a scan's filename should not change regardless of the type of file, unless the file has been edited for a specific purpose.

For example:
KAUFMAN_B01F12_003.tif
Original Scan / Archival Master
KAUFMAN_B01F12_003.jpeg
Access file created by saving a copy of the original scan as a jpeg in an image editing program. 
KAUFMAN_B01F12_003_web.jpeg

KAUFMAN_B01F12_003_print.psd
Copy of the access jpeg file that has been saved for web (reduced resolution, size, or smaller colour space, etc.)

A copy of the original scan that has been edited in Photoshop for use in a publication or hard copy print.

I avoid using the word "copy" in file names because it does not tell me about the purpose of the file or why it was created. Some image editing software will add the word "copy" to a file's name if the original file name is already taken to avoid a naming conflict. If you want the file to replace the file that has the same name, you can choose to overwrite the original file when saving the new file with the same name. If you are making different adjustments to a file and want to be able to compare the iterations, use the suffix '_version01' or '_v01'.

\section{Folder Structure}

Creating a file organization system, also called a directory structure, is just as important as creating a file-naming system. There are numerous ways you can organize folders, but again, the most important thing is to be consistent. The main folder should be the broadest, encompassing your whole archive. The next set of folders inside the main folder could be different collections or different types of materials. It really depends on how your materials are organized. Perhaps everything is in boxes and you want to keep everything in each box together. In that case, your second level folders could be BOX_01, BOX_02, BOX_3, etc. The third set of folders specifies the document title or group of documents, for example the pages of an album. The following diagram is the 
folder structure I use, use mirrors the main categories I created while cataloguing the materials (Section 1.5). I separate the file types into folders within each Document Title folder: MASTER_TIFFS for the high-quality original scans, and ACCESS_JPEGS for the smaller derivative JPEG files I create that can be used only, shared, viewed, and edited.

Red $=$ Main Folder

Orange $=$ Material Categories $/$ Types

Green $=$ Document Title

Blue = File Type $/$ Use

Purple $=$ Files

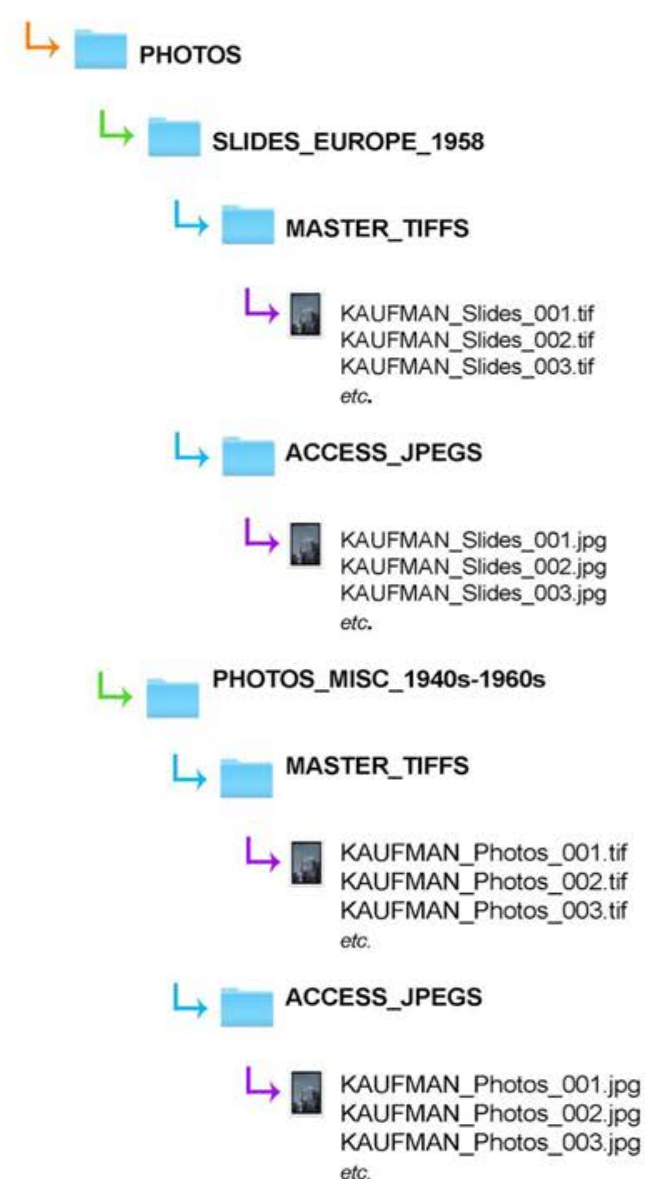

etc.

$\llcorner\square$ ALBUMS

$\hookrightarrow$ ALBUM_PERU_1970s

$\hookrightarrow$ ALBUM_JAPAN_1940s

4 ALBUM_GEM_TINTYPE_1880s

$\hookrightarrow$ OBJECTS

ᄂ BOUND DOCS

ᄂ — LOOSE DOCS 


\subsection{Hard Drives and Backups}

\section{Hard Drives}

A hard drive is the device inside your computer to which all data is written. All the photographs you save, programs you install, all of the programming files needed for your computer to work properly are recorded onto the hard drive. Hard drives are also referred to as memory, although there are different types of memory, ROM and RAM. ROM is the hard drive described above, and RAM, or random access memory, is separate storage used for the short term storage of data needed to process immediate commands and temporary data while you are using the computer. I liken memory to a table. RAM is the size of the table, and ROM is the amount of things you can put on top of the table. More RAM means a bigger table, and more ROM means a larger vertical space on which to pile your data. Defragmenting a hard drive is like rearranging the stuff on the table into neater stacks that fit together better and free up space in which to put more stuff. Thankfully, most hard drives now no longer need to be manually defragmented.

Not many people stop to consider what the 'degradation' of something digital actually entails. Is it possible? Do digital files and hard drives go bad? YES! Unfortunately they do, and it is not uncommon. If you have ever tried to plug in an old hard drive ('old' in this case might be one that is more than 5 years old) into your computer, you may have encountered corrupted files or versions of firmware [define] that are no longer compatible with your younger computer. The first hard drive I ever purchased was 60 gigabytes, about \$200 USD, and was the technological pride and joy of my 15-year-old self. Several years ago, I dug the old drive out of a box in the garage, and copied all of the data from it onto a newer drive because I knew the original hard drive did not have much time left. Hard drives are physical things that degrade just like other physical things. The most common type of hard drive available is SATA (Serial Advanced 
Technology Attachment). ${ }^{24}$ Over the last few years they have started to be replaced by solid-state drives (SSD), a welcome change to most nerds like myself, despite their higher price tag. As you might guess, SATA drives differ from solid state drives in one big way: they are not solid; they flow, or in this case, spin. That whirring sound you hear when you plug a hard drive into your computer is the internal disk spinning, (hopefully) being scanned by a laser, and the data being transmitted to your computer through a USB cable. Because a SATA drive has moving parts, it is susceptible to damage. If you've ever dropped a hard drive, you might be familiar with this. A dropped hard drive often becomes a better paper weight than device for digital data storage.

Hard drives, especially spinning SATA drives, do not last forever. A SATA drive that is constantly left plugged into a computer, spinning $24 / 7$ will absolutely have a shorter lifespan than one that is unplugged when not in use. Of course it is often beneficial to leave a hard drive plugged in and running if you automatically back-up your computer files to the drive, but if not, I like to think of it like a lightbulb that is never turned off. If you do not need the light, you are both wasting energy and shortening the bulb's useful life. This is a big advantage offered by solid state drives. They have no moving parts, so there is not much that will break easily. They are definitely not indestructible, but they are much more robust. Popular hard drive brands include Western Digital, Seagate, LaCie, G-Tech, Toshiba, and Verbatim.

\section{PORTABLE vs. DESKTOP HARD DRIVES}

A portable hard drive is one that is 'bus-powered', meaning that it does not need to be plugged into an external power source to function. It draws its power directly from the computer into which it is plugged. Portable hard drives are also usually physically smaller than their desktop counterparts. The drive disks themselves are 2.5 " in

24. SATA actually refers to the type of high-speed wired connection between the drive disk and the computer. 
diameter, the same size drives as those that are used in laptop computers. ${ }^{25}$ Common portable hard drive sizes are 500GB, 1TB, 2TB, 4TB, and 5TB.

Currently anything over 5TB requires an external power source to run, meaning it will have to be plugged into a power outlet as well as into the computer. ${ }^{26}$ These are called 'desktop' hard drives. Because they need an external power source, they are less portable and will likely live in one place (perhaps on the top of your desk, hence the name). Desktop hard drive disks are 3.5" in diameter and are the same kind of hard drives as those in desktop computers.

\section{Raid Systems}

RAID stands for Redundant Array of Independent Disks, and is a great way to get serious about backing up your files, although not necessary. RAID systems are more expensive than single hard drives for several reasons. A RAID system is two or more hard drives enclosed together in a larger housing. Consumer RAID systems generally range from the size of a paperback novel to the size of a shoebox and they add more protection for your files by distributing the data you would normally put on a single drive across multiple drives. This way, if one of the hard drives encounters a problem (if a file is damaged or corrupted and is no longer readable), it will repair the data by compiling portions of the data across the other drives to reconstruct the damaged file. There are different types of RAID systems, RAID 0, RAID 1, RAID 2, etc. up to RAID 10. Each of these RAID versions uses a different model for distributing the data across the interconnected hard drives. It may seem paranoid to worry about corrupted files if your hard drive is just sitting on your desk plugged into your computer, and not doing much else. However, hard drives can be finicky and can encounter problems for no apparent reason, so if you have a lot of irreplaceable data stored on your hard drive, you might

25. You can remove the hard drive from a laptop and put it into a drive case for use as an external, portable hard drive. l've done this when a laptop stopped functioning and was not worth repairing, but still had a fully-functional internal hard drive.

26. Desktop hard drives are also available in smaller sizes, but portable drives 4TB and under are now more common and desktop hard drives of the same size. 
want to think about upgrading to a RAID system. When it comes to hard drives, it is definitely better to be safe than sorry. Regardless of whether or not you use a single hard drive or a RAID system, backups are essential.

\section{Backups}

To ensure that your data is safe, you must back it up. There are different ways to back up your files and the best rule of thumb to follow is the 3-2-1 rule, coined by photographer Peter Krogh ${ }^{27} .3$ copies in 2 different media with 1 copy located offsite. For example, you could store your data on an external hard drive, have an identical hard drive as a backup, with a third copy stored on a cloud service.

EXAMPLE 1:

EXAMPLE 2:

EXAMPLE 3:
Copy 1: External Hard Drive

Copy 2: External Hard Drive

Copy 3: Cloud Storage

Copy 1: Internal Hard Drive (your computer) ${ }^{28}$

Copy 2: External Hard Drive

Copy 3: Cloud Storage

Copy 1: External Hard Drive

Copy 2: CDs or DVDs ${ }^{29}$

Copy 3: Cloud Storage

27. Peter Krogh, "Backups," The DAM Book: Digital Asset Management For Photography, http://thedambook.com/dyp/backups/ (Accessed June 13, 2018).

28. Depending on how much data you have, you may not have enough room on your computer to store a complete copy of all your files (your archival scans, for example).

29. If you can, avoid backing up on CDs and DVDs. CD and DVD readers are often no longer built into computers, and the technology may become completely obsolete in the next decade or less. It is much easier and less expensive to store a copy of your data on another hard drive rather than burn files in 4GB chunks onto dozens or hundreds of disks. Additionally, CDs and DVDs scratch very easily, and once damaged, your data will no longer be readable. 
It is important to understand that social media is NOT a viable backup for your files! Uploading photographs to Facebook may be a good way to share with family and friends, but in addition to Facebook's murky copyright policies, files are drastically compressed and resized when uploaded. Just don't do it. Some well-respected cloud storage services include Dropbox, Box, iCloud, Google Drive, Amazon Glacier, Carbonite, SpiderOak, BackBlaze, and SugarSync. Most of these services have small storage tiers available for free or for a trial period, while others like Amazon Glacier require a very low monthly fee to back up your data, but charge you additional fees to recover your data. This type of storage is good if you plan to retrieve data from the cloud only in an emergency. Other services like Dropbox, Box, iCloud, and Google Drive are designed for easy accessibility, allowing you to upload, download, and share data as needed at no extra charge.

While cloud storage is technically 'off-site' because it is not located on your desk, you may also want to store an external hard drive copy of your data in another location, such as a bank safety deposit box, your work office, or a relative's house, as long as you remember to update that copy regularly if you have new data. Having an off-site copy is important in case of fire, natural disaster, burglary, etc. It may also be worthwhile to store the external hard drives you keep at home in a fire safe. Again, this may seem paranoid, but when thousands of digital files and photographs are at stake, it's better to be safe than sorry! 
SECTION 2: DIGITIZATION 


\subsection{Digitization Equipment}

A key component to digitizing analog materials is, naturally, the digitization equipment. Different types of equipment are better suited to different types of materials while some work well with a range of materials, and it can sometimes be difficult to know which is best suited to your needs and your budget. The good news is that if you have access to a computer, it is possible to digitize the majority of your materials for less than $\$ 200$. I used an Epson V370 flatbed scanner, which costs about $\$ 150$, to digitize photographs, slides, and film, and then processed the files in Gimp, a free, open-source imaging program. More expensive equipment and paid programs like Adobe Bridge, Photoshop, Lightroom will allow you to process images more quickly and efficiently. At the very minimum, you need a computer and scanner to digitize your materials. I highly recommend also backing up to an external hard drive. For more information about hard drives, refer to Section 1.8 .

\section{HARDWARE:}

Computer

Flatbed Scanner ${ }^{30}$

Digital Camera

HoverCam SOLO 8Plus Document Scanner ${ }^{31}$

External Hard Drive ${ }^{32}$

Colour-checker

Copy Stand

Table-top Lights

Table-top or Full-sized Tripod

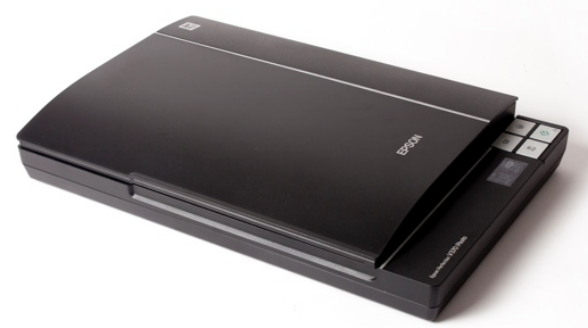

Flatbed Scanner (Items not to relative scale)

30. See Section 2.3.

31. See Section 2.7.

32. See Section 1.9.

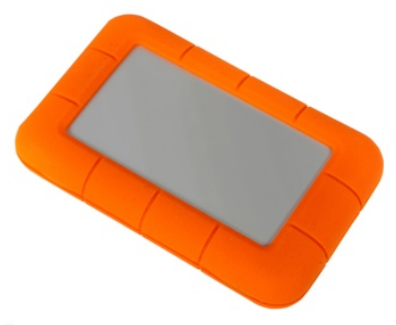

External Hard Drive

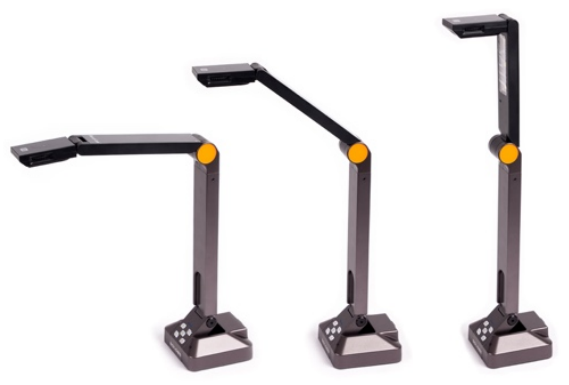

HoverCam Solo 8Plus 


\section{SOFTWARE:}

With a free Google account, you have access to Google sheets, which is the company's answer to Microsoft Excel. Adobe Photoshop, Lightroom, and Bridge can be purchased from Adobe for $\$ 9.99$ USD per month. Gimp is a free software that, while not as elegant as Photoshop, will allow you to do the post-processing you will need.

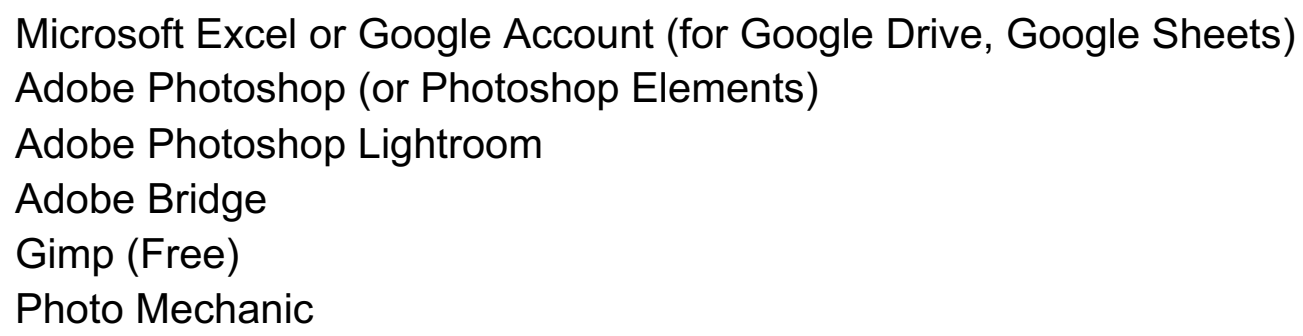

\section{TYPES OF SCANNERS}

There are many types of specialized scanners available today, but the most common types used in archives and museums are flatbed scanners and overhead book scanners. The sheer number and brands of scanners available at different price points can be overwhelming, so it is best to start by considering what you will be using the scanner for. That being said, I have used numerous models of Hewlett-Packard, Canon, Sony, and Epson scanners over the years (in addition to others like Hasselblad, Imacon, and Plustek that are out of the average price-range) and have found that Epson scanners are the most reliable and provide the best quality output. Please refer to the chart below for additional information about a variety of Epson flatbed scanners.

\section{Flatbed Scanners}

Flatbed scanners digitize materials by scanning the document pixel row by pixel row while the document is laying face-down on a piece of clear glass. Here are some things to consider when buying a flatbed scanner:

Optical Resolution or Effective Resolution describes the number of pixels a scanner can see with its own sensors. Items scanned at this resolution will have the most accurate 
pixel data, so naturally the higher the optical resolution a scanner is capable of, the better quality the scanner (and the higher the price tag).

Max Resolution or Interpolated Resolution describes the maximum resolution the scanner can output when using algorithms to mathematically increase the number of pixels produced. Pixels generated by interpolation (the increasing of output pixels) are not as accurate as those captured directly by the scanner sensors.

Max Document Size is the dimensions of the scanner bed. If you know you will be scanning lots of larger documents, consider a larger flatbed scanner. Additionally, if you have lots of slides or film to scan, going with a larger scanner will save you lots of time since you will be able to scan more images at once.

Colour Bit Depth refers to the number of colour values a scanner can capture. Some lower-end scanners can only capture 24-bit colour images (16.7 million values) whereas professional imaging scanners can capture 48-bit colour images (281 trillion), which is ideal for scanning film.

Flatbed Scanner Guide ${ }^{33}$

\begin{tabular}{|c|c|c|c|c|c|c|}
\hline Scanner & $\begin{array}{l}\text { Optical } \\
\text { Res. } \\
\text { (dpi) }\end{array}$ & $\begin{array}{l}\text { Max Res. } \\
\text { (dpi) }\end{array}$ & $\begin{array}{l}\text { Max Doc. } \\
\text { Size }\end{array}$ & $\begin{array}{l}\text { Colour } \\
\text { Bit } \\
\text { Depth }\end{array}$ & Formats & $\begin{array}{l}\text { Price } \\
\text { (CDN) }^{34}\end{array}$ \\
\hline Epson V370 & 4800 & 12800 & $8.5^{\prime \prime} \times 11.7 "$ & 48-bit & $\begin{array}{l}\text { Slides ( } 4 \text { ) } \\
35 \mathrm{~mm} \text { ( } 12 \text { frames) }\end{array}$ & $\$ 150$ \\
\hline Epson V550 & 6400 & 12800 & $8.5^{\prime \prime} \times 11.7 "$ & 48-bit & $\begin{array}{l}\text { Slides ( } 4 \text { ) } \\
35 \mathrm{~mm} \text { ( } 12 \text { frames) }\end{array}$ & $\$ 250$ \\
\hline Epson V600 & 6400 & 12800 & $8.5^{\prime \prime} \times 11.7 "$ & 48-bit & $\begin{array}{l}\text { Slides }(4) \\
35 \mathrm{~mm}(12 \text { frames }) \\
120 / 220 \mathrm{~mm} \\
(1 @ 6 \times 20 \mathrm{~cm})\end{array}$ & $\$ 280$ \\
\hline
\end{tabular}

33. "Epson Scanners," EPSON, https://epson.ca/scanners (Accessed June 16, 2018).

34. Prices are based on Spring 2018 averages found on Amazon.ca and B\&H Photo. 


\begin{tabular}{|c|c|c|c|c|c|c|}
\hline $\begin{array}{l}\text { Epson } \\
\text { V700/750 }\end{array}$ & 6400 & 12800 & $8.5 "$ x 11.7" & 48-bit & $\begin{array}{l}\text { Slides (12) } \\
35 \mathrm{~mm}(18 \text { frames) } \\
120 / 220 \mathrm{~mm} \\
(2 @ 6 \times 20 \mathrm{~cm}) \\
4 \times 5 \text { film }(2)\end{array}$ & $\$ 1000$ \\
\hline $\begin{array}{l}\text { Epson } \\
\text { V800/850 } \\
\text { (Newest V } \\
\text { Model) }\end{array}$ & 6400 & 12800 & $8.5 "$ x 11.7" & 48-bit & $\begin{array}{l}\text { Slides }(12) \\
35 \mathrm{~mm}(18 \text { frames }) \\
120 / 220 \mathrm{~mm}(6 \times 20 \mathrm{~cm}) \\
4 \times 5 \text { film (1) }\end{array}$ & $\$ 1100$ \\
\hline $\begin{array}{l}\text { Epson } \\
10000 X \mathrm{~L}\end{array}$ & 2400 & 12800 & $12.2 " x$ 17.2" & 48-bit & $\begin{array}{l}\text { Slides }(30) \\
35 \mathrm{~mm}(18 \text { frames }) \\
120 / 220 \mathrm{~mm}(3-6 \times 20 \mathrm{~cm}) \\
4 \times 5 \text { film }(4)\end{array}$ & $\$ 2000$ \\
\hline $\begin{array}{l}\text { Epson } \\
11000 X \mathrm{~L}\end{array}$ & 2400 & 12800 & $12.2 " x$ 17.2" & 48-bit & $\begin{array}{l}\text { Slides }(30) \\
35 \mathrm{~mm}(18 \text { frames }) \\
120 / 220 \mathrm{~mm}(3-6 \times 20 \mathrm{~cm}) \\
4 \times 5 \text { film }(4)\end{array}$ & $\$ 2500$ \\
\hline $\begin{array}{l}\text { Epson } \\
12000 X \mathrm{~L} \\
\text { (Newest XL } \\
\text { Model) } \\
\end{array}$ & 2400 & 12800 & $12.2 " x$ 17.2" & 48-bit & $\begin{array}{l}\text { Slides }(30) \\
35 \mathrm{~mm}(18 \text { frames }) \\
120 / 220 \mathrm{~mm}(3-6 \times 20 \mathrm{~cm}) \\
4 \times 5 \text { film }(4)\end{array}$ & $\$ 4600$ \\
\hline
\end{tabular}

\section{Overhead Book Scanners}

Overhead book scanners are designed to easily digitize bound documents. They usually have either a book cradle and 2 cameras, or built-in technology to counter-act the distortion created by angled pages laying either in a book cradle or on a flat surface. They are designed to be fast, allowing the user to quickly scan large volumes, and combining the scans into PDF documents. Unfortunately, one of the major drawbacks of most overhead book scanners is that they do not capture in a TIF format. For example, the Fujitsu ScanSnap SV600 scanner, popular among genealogical and historical societies, can only scan as PDF or JPEG, with a maximum resolution of $1200 \mathrm{dpi}$.

\section{Document Scanners}

Document Scanners are scanners designed to scan lots of paper documents very quickly. There are different types of document scanners: sheet-fed, portable, or 
overhead (like book scanners). They can be very appealing for their low price point and high-volume, decent quality output, but when it comes to archival digitization, they fall short. Most do not scan as TIFFs and have a maximum resolution of $600 \mathrm{dpi}$. It is also not advisable to feed old and delicate materials through a sheet-fed scanner as the risk of physical damage to the materials is very high. However, imaging companies now manufacture high-speed document scanners, like Kodak's i4250, ${ }^{35}$ that can scan a wide range of paper weights and utilize advanced technology to detect even the slightest paper jam to immediately stop the scanner before the document in question is damaged. These kinds of scanners are being cautiously tested by larger organizations, and have relatively large price tags (around $\$ 5,000$ to $\$ 8,000$ ).

\section{Drum Scanners}

Drum scanners are high-end scanners used to digitize film. They work by slowly sliding a strip of film over a cylindrical drum, and then scanning the film pixel row by pixel row as it crosses the apex of the drum, ensuring that the film is as flat as possible when scanned. Film scanned on a flatbed scanner does not lay completely flat, so the scans are not as sharp as those produced by a drum scanner. Consequently, drum scanners are extremely expensive, often well into the five-figure price range.

\section{DIGITAL CAMERAS}

Most consumer and professional cameras will work well for digitizing documents. The only real requisite is that they be able to capture images in either a RAW file format, or in some cases TIFF, in addition the usual JPEG format. When scanning using a digital camera, you will need to process the RAW files through Lightroom or a comparable image processing program in order to export the files as TIFFs. Even when you do this, though, it is a good idea to keep the original RAW files in addition to the exported TIFFs, just in case you need to return to the original file to make adjustments. For more information about processing in Lightroom, see Sections 3.6 and 3.7. For more information about RAW file formats, refer to the glossary.

35. "Kodak 14250 Specs," CNET, https://www.cnet.com/products/kodak-i4250-documentscanner/specs/ (Accessed June 14, 2018). 
Here is a flowchart I have created to help you decide what type of scanner is best for you:

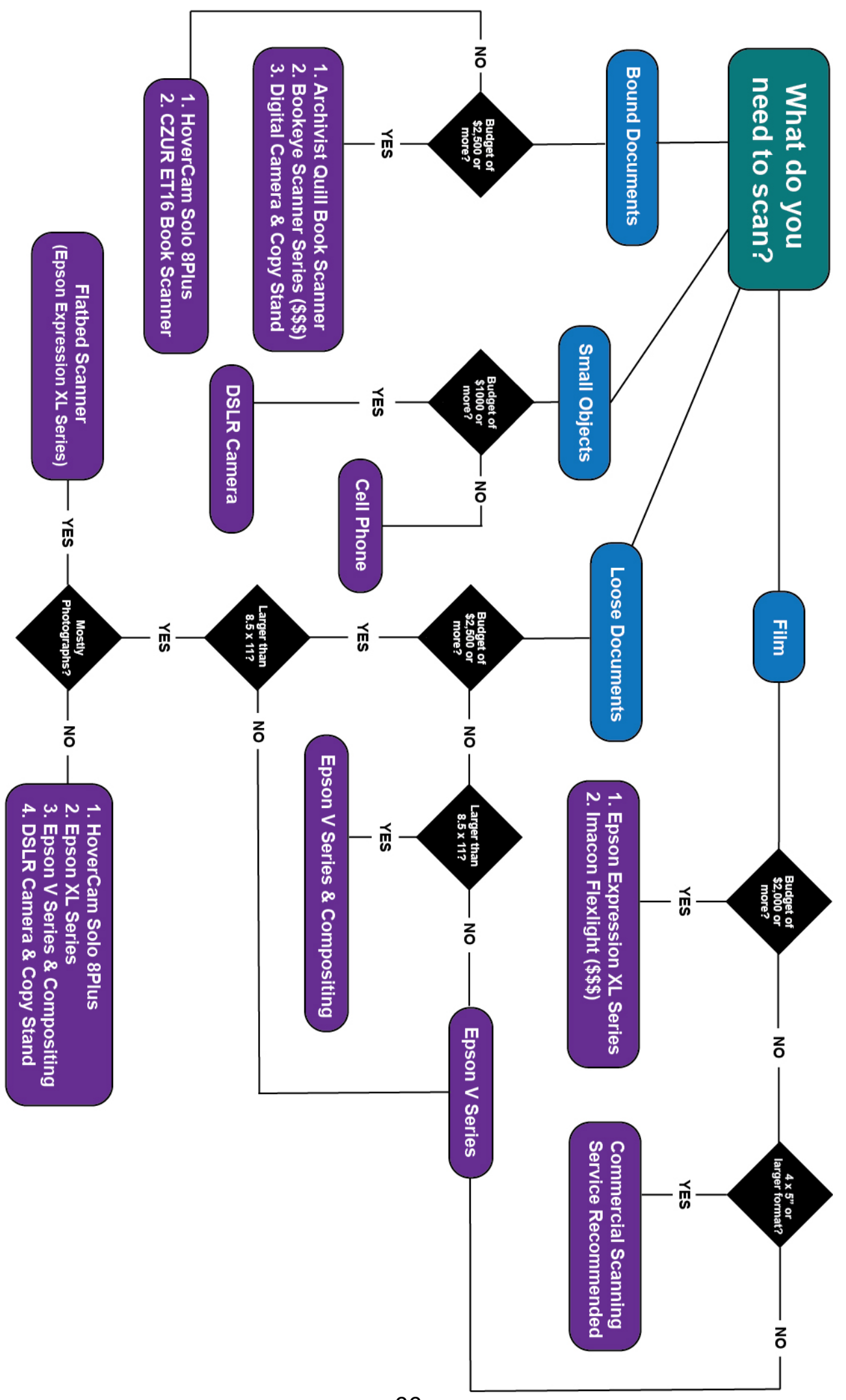




\subsection{Scanning Reflective Documents}

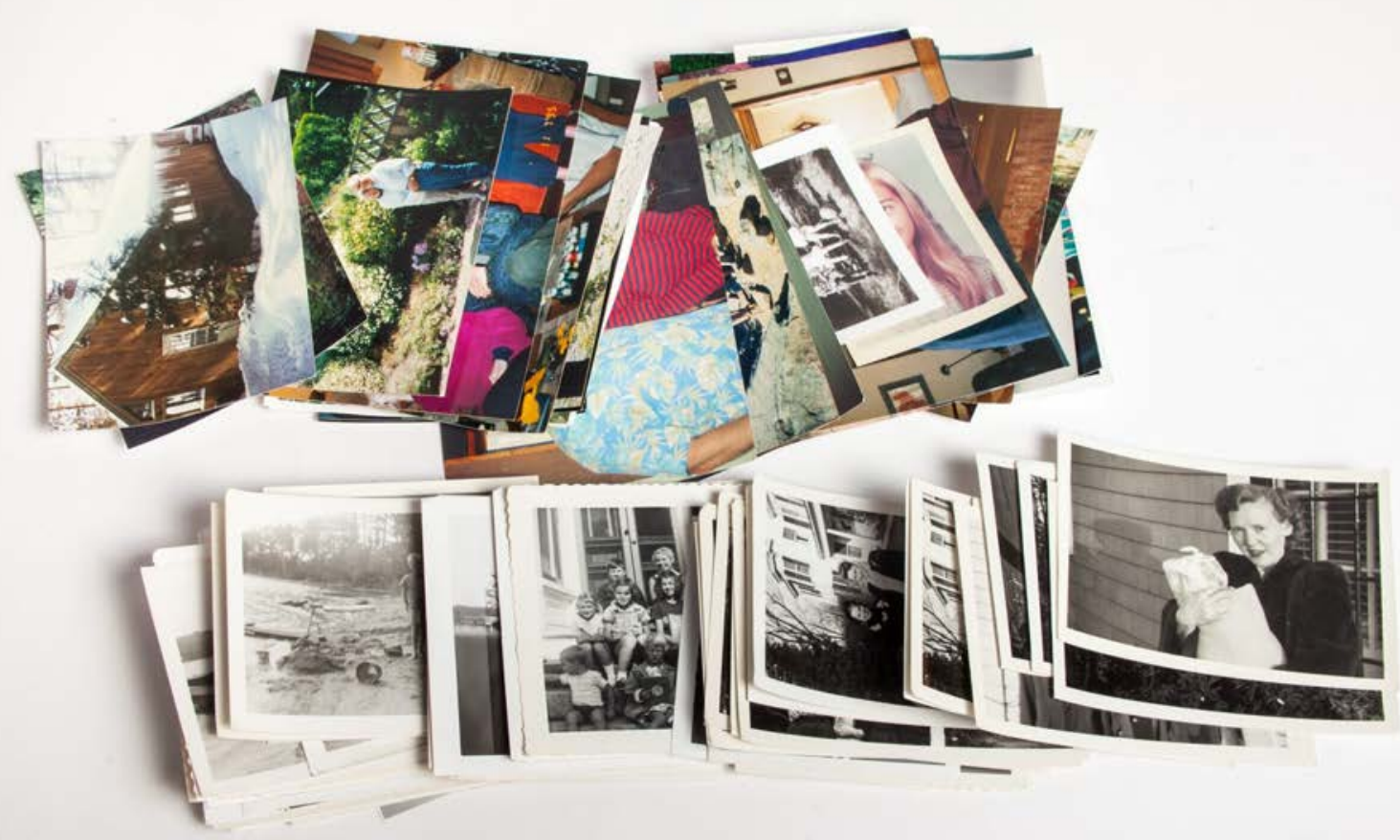

Most items in your collection are likely reflective documents, meaning they are not transparent. These can be anything from letters, deeds, birth certificates, printed photographs, drawings, and the like. Many organizations and institutions choose to digitize reflective material, regardless of their sizes, using a digital camera, studio lights, and a copy stand. However, while this is definitely a great method, it can come with a hefty price tag.

The best way to digitize these kinds of documents is to scan them using a flatbed scanner. There are many great flatbed scanners (which can also digitize film and slides) on the market with the majority manufactured by Epson, Canon, and HP. I generally favour Epson scanners as in my experience they seem to be more reliable and produce better quality scans than their Canon and HP counterparts. For more information about scanners, refer to Section 2.2 Equipment and Hardware 


\section{What You Will Need:}

- Flatbed scanner (I used an Epson Perfection V370 Photo)

- Computer with the Epson Scan software installed.

- A piece of black or dark grey mat board (or other rigid black material) slightly larger than the size of the scanner bed. I used a $9 \times 12$ " canvas board covered with a piece of black self-adhesive vinyl purchased from my local craft store.

- External Hard drive (for backing up)

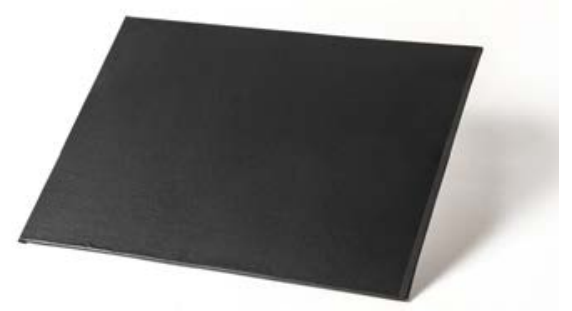

- Photoshop, Photoshop Elements, Lightroom, or Gimp
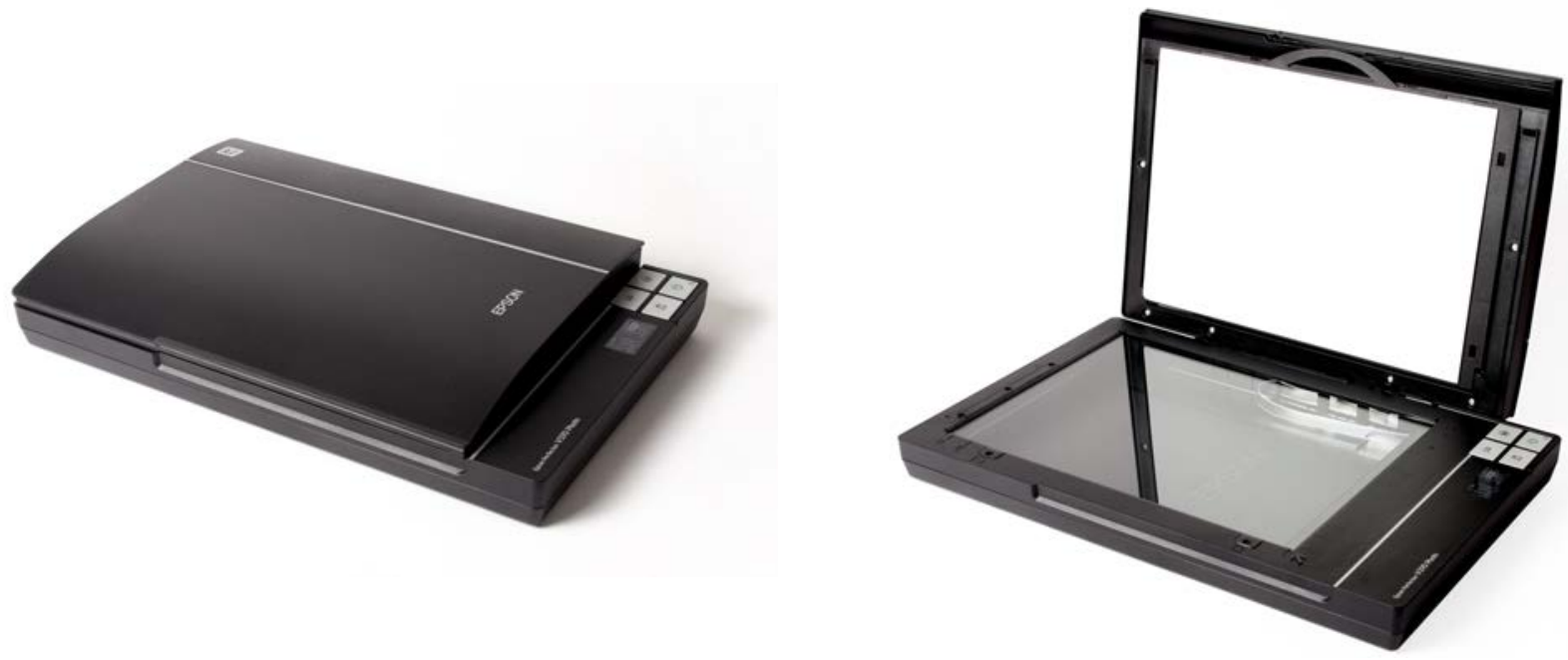

1. Connect the scanner to the computer and turn the scanner on.

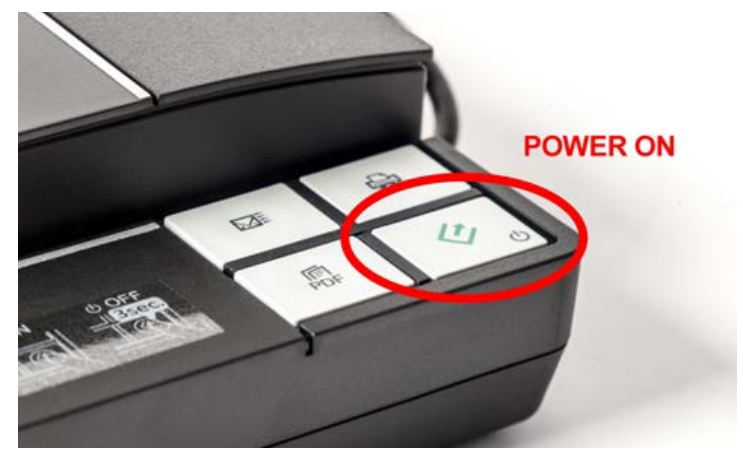


3. Make sure the scan mode is set to PROFESSIONAL.

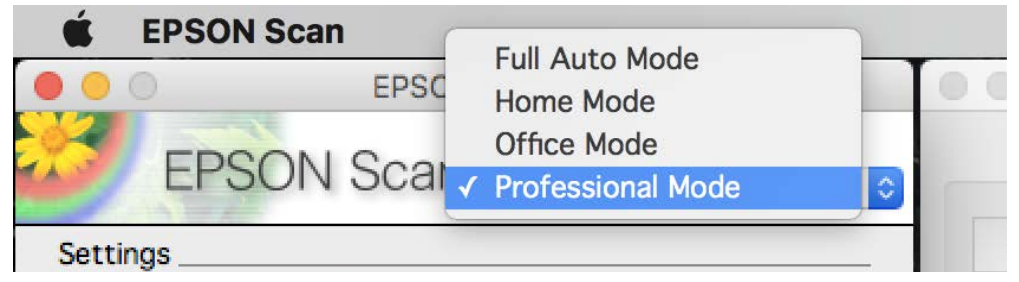

4. Set document type to REFLECTIVE.

5. Select DOCUMENT TABLE as the document source.

6. Set Auto Exposure Type to РНОТО.

7. Set Image Type to 24-BIT COLOR.

8. The RESOLUTION at which you will scan your documents depends on the size of the document you are scanning. As a rule of thumb, the smaller the document the higher the resolution value should be. Archival standards recommend a long edge of around 4,000 to 5,000 pixels, with up to 10,000 pixels for aerial negatives that will likely need to be blown up to large sizes. I lean towards 5,000 pixels for most materials. So, if your

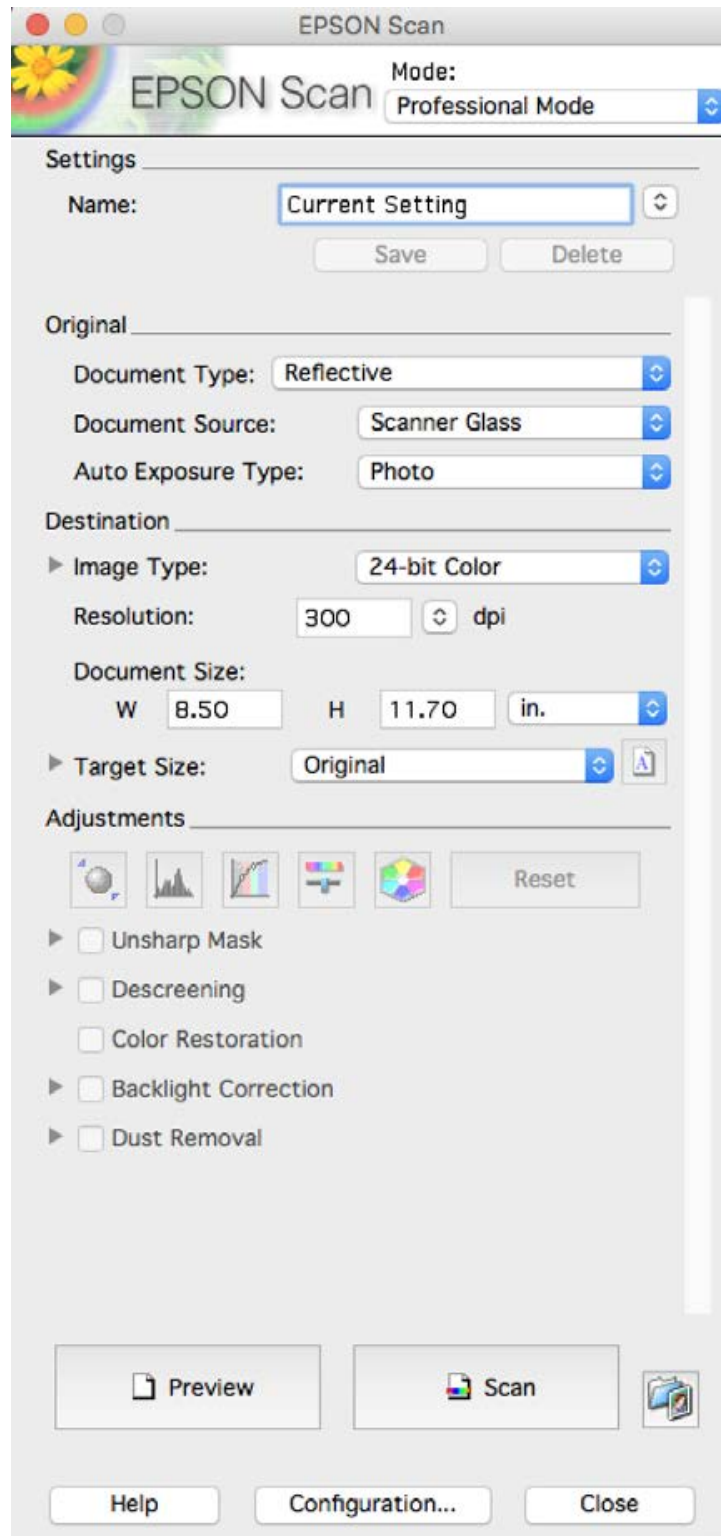


document is 2 inches on the long side, divide 5,000 by 2 and that will be the resolution at which you should scan.

\section{Example:}

a. If you are scanning a $5 \times 7$ inch photograph, you should scan at $720 \mathrm{dpi}$ (because $5,000 \div 7=$ approximately 714 ). The resulting scan will be 3,600 $x 5,040$ pixels, not including any border you include.

b. If you are scanning an $8 \times 10$ inch photograph, scan at 500 or $600 \mathrm{dpi}(5,000 \div 100=500)$. I generally stick to the options provided in the application's drop down menu, but you can also input your own dpi values, although it is not recommended that you scan at a resolution less than $300 \mathrm{dpi}$.

When deciding your target dpi, it is also good to consider the content of the document. If the document is a photograph with lots of small people, a class photo for example, you will likely want to scan at a slightly higher resolution to capture more of the detail. If the document is largely blank, or without small details, a typed letter of portrait of a single individual for example, scanning at a lower dpi is fine.

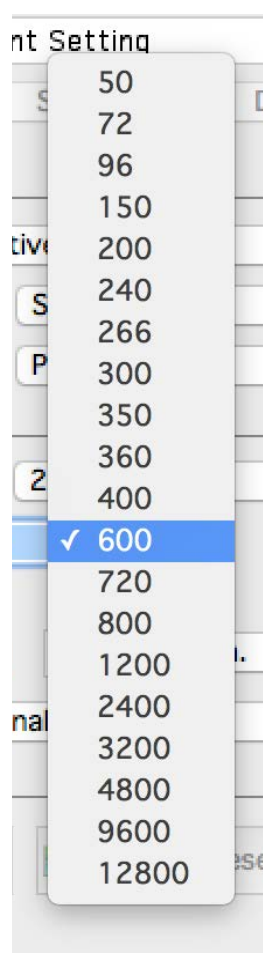

\section{Example:}

Each of these photographs is $2.5 \times 3.5$ inches. If I want approximately 5,000 pixels on the long end, I should scan at $1400 \mathrm{dpi}(5,000 \div 3.5=1428)$. However, one image-the group portrait-has a considerable amount of detail, while the other-the landscapedoes not. 

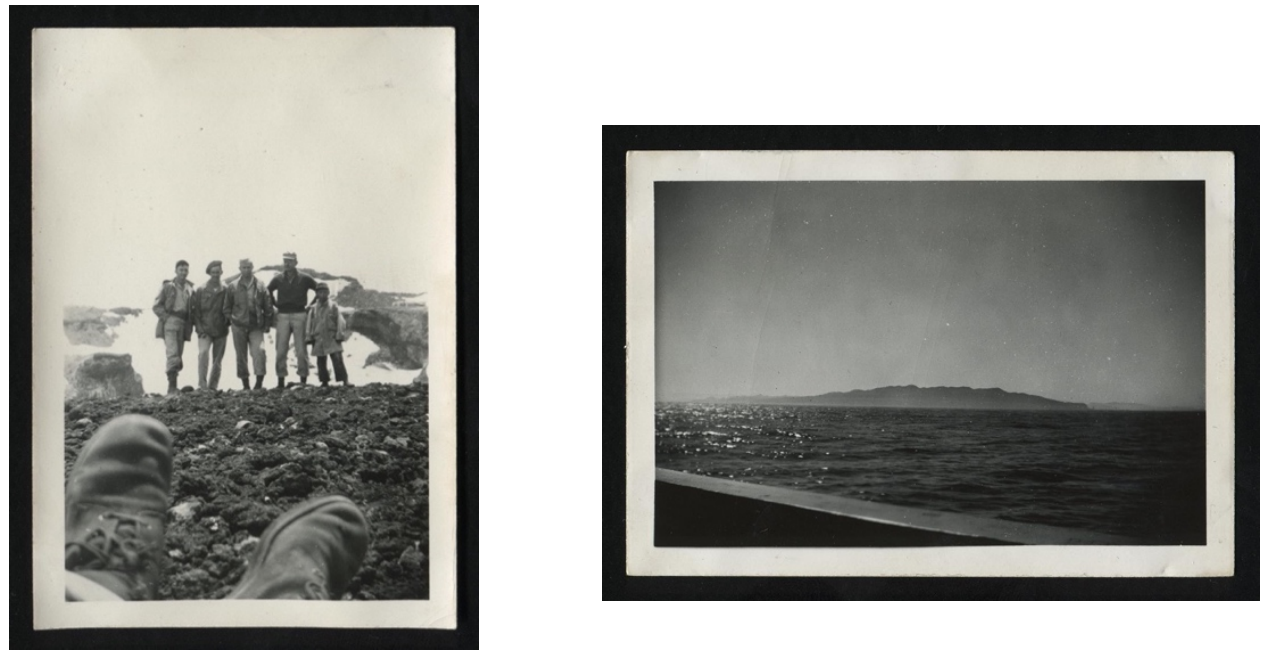

9. Place the document you want to scan face down on the glass scanner bed. If the document is curled slightly, the black mat board to other material you place over it should help to flatten it.

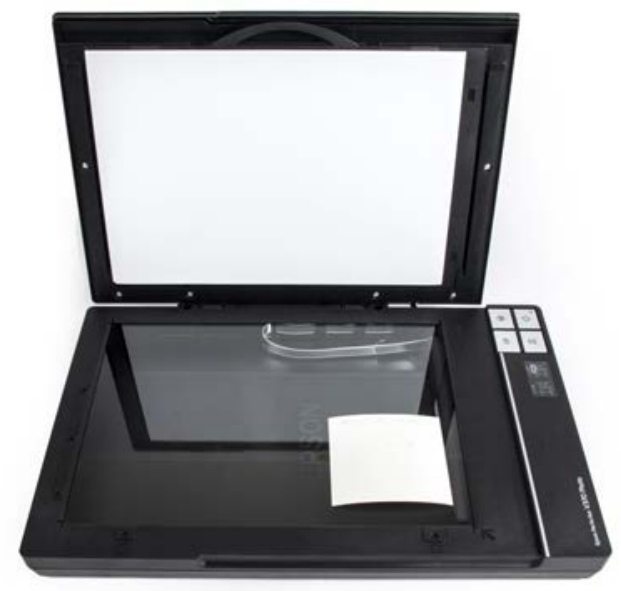

You may want to cut the mat board to be slightly smaller than the glass bed so that it can sit flush against the glass.
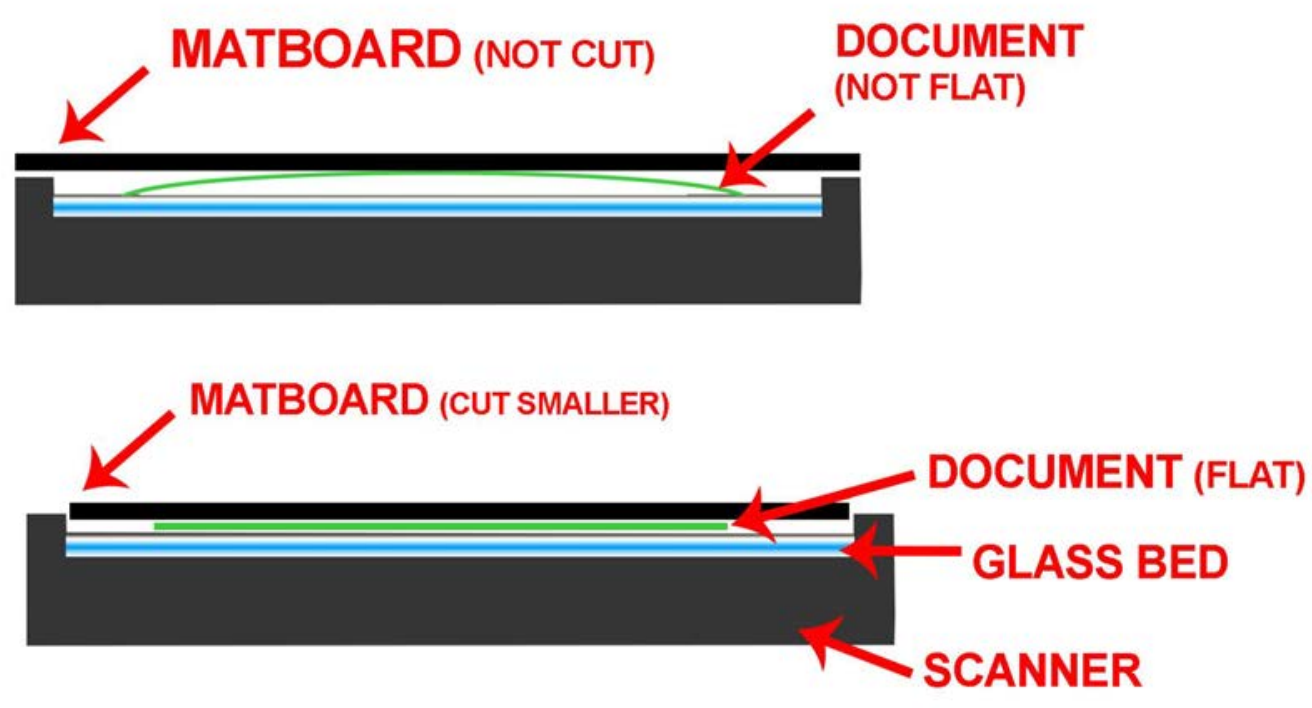
10. Click PREVIEW. The scanner will now display a preview of the document.

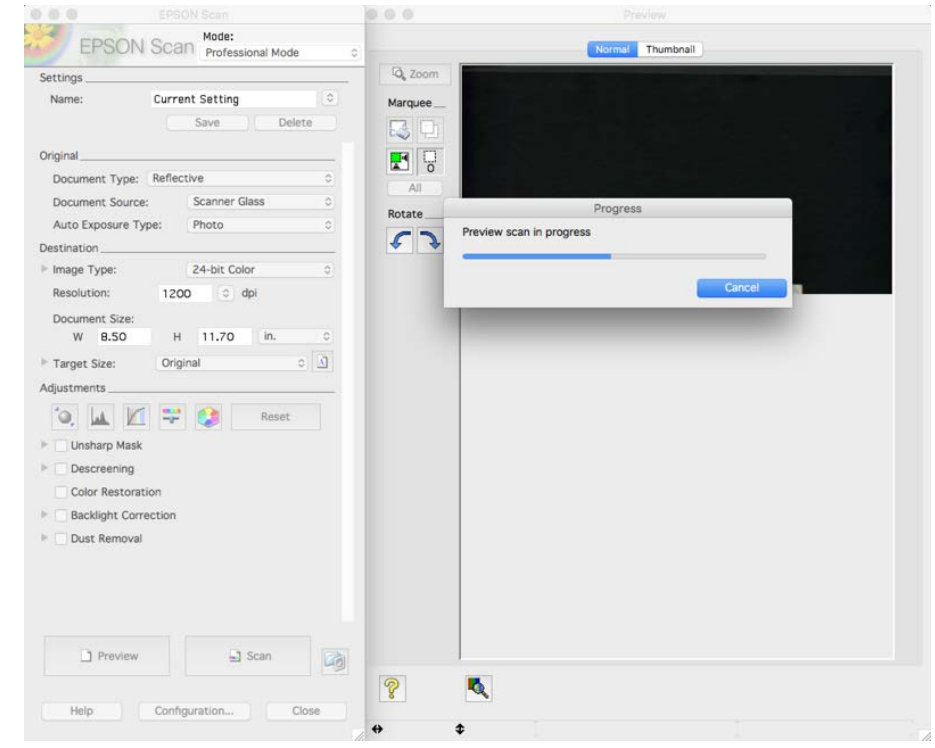

11. Using your mouse cursor, draw and drag a selection marquee around your document to select the area you want to scan. If you do not select the area, the scanner will scan the full scanning bed, which unless you are scanning a document that is the full size of the scanner bed, will scan a lot of unneeded space a create an unnecessarily large file.

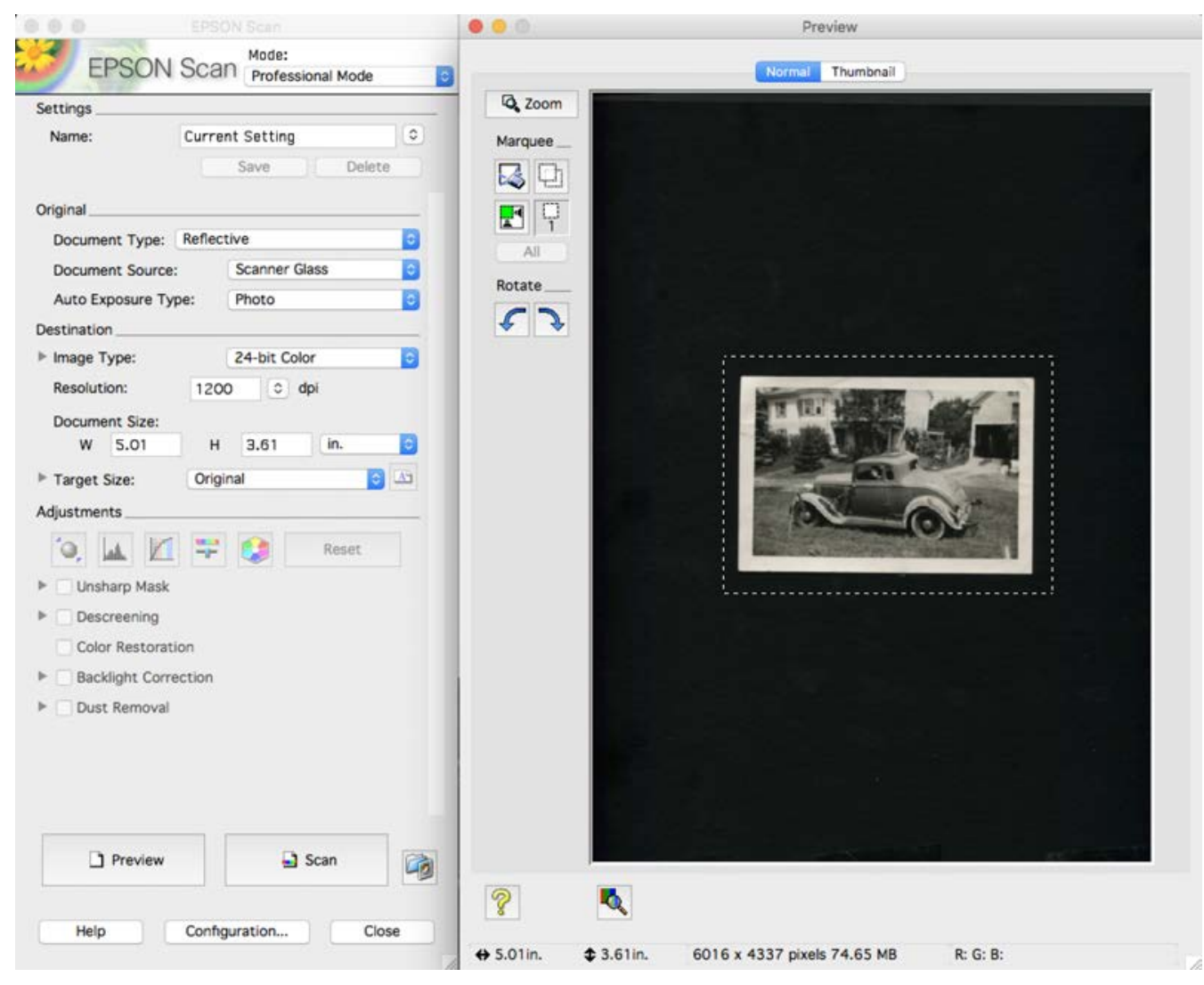


There is an option to 'automatically locate the image', but I do not use this function because, per common archive and museum practice, I like to leave a $0.5 \mathrm{~cm}$ or so border around the image. This border helps define the edge of the document, ensuring that it is not cropped, and can help make thinner materials clearer and more legible

To delete the marquee you have drawn, click the ERASER icon.

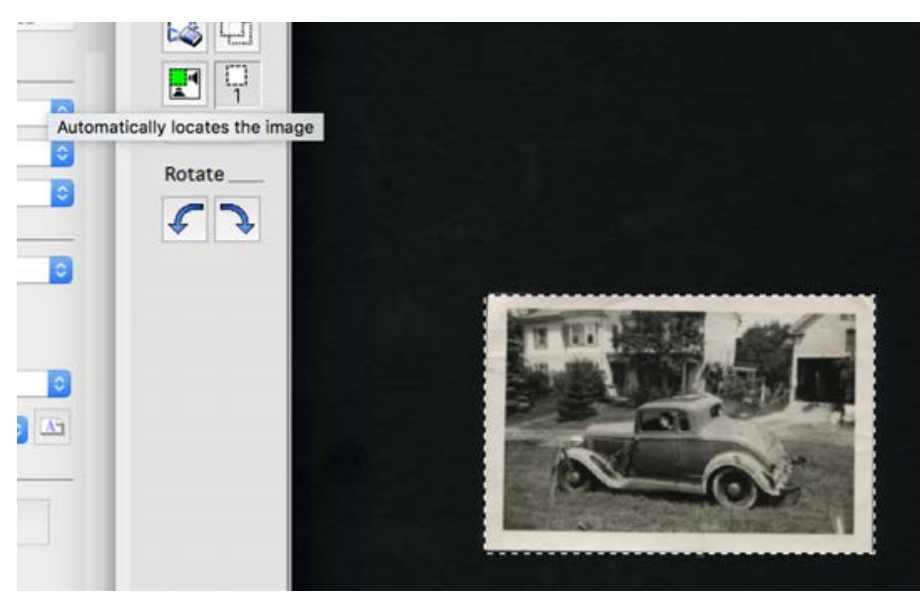

To scan multiple documents at once (several small photos, for example), place the documents face down on the scanner, click PREVIEW, and then draw individual marquee boxes around each image. Make sure that all the boxes are showing up as dashed lines, indicating that they are active scan areas. If not all boxes are selected, click the ALL button.
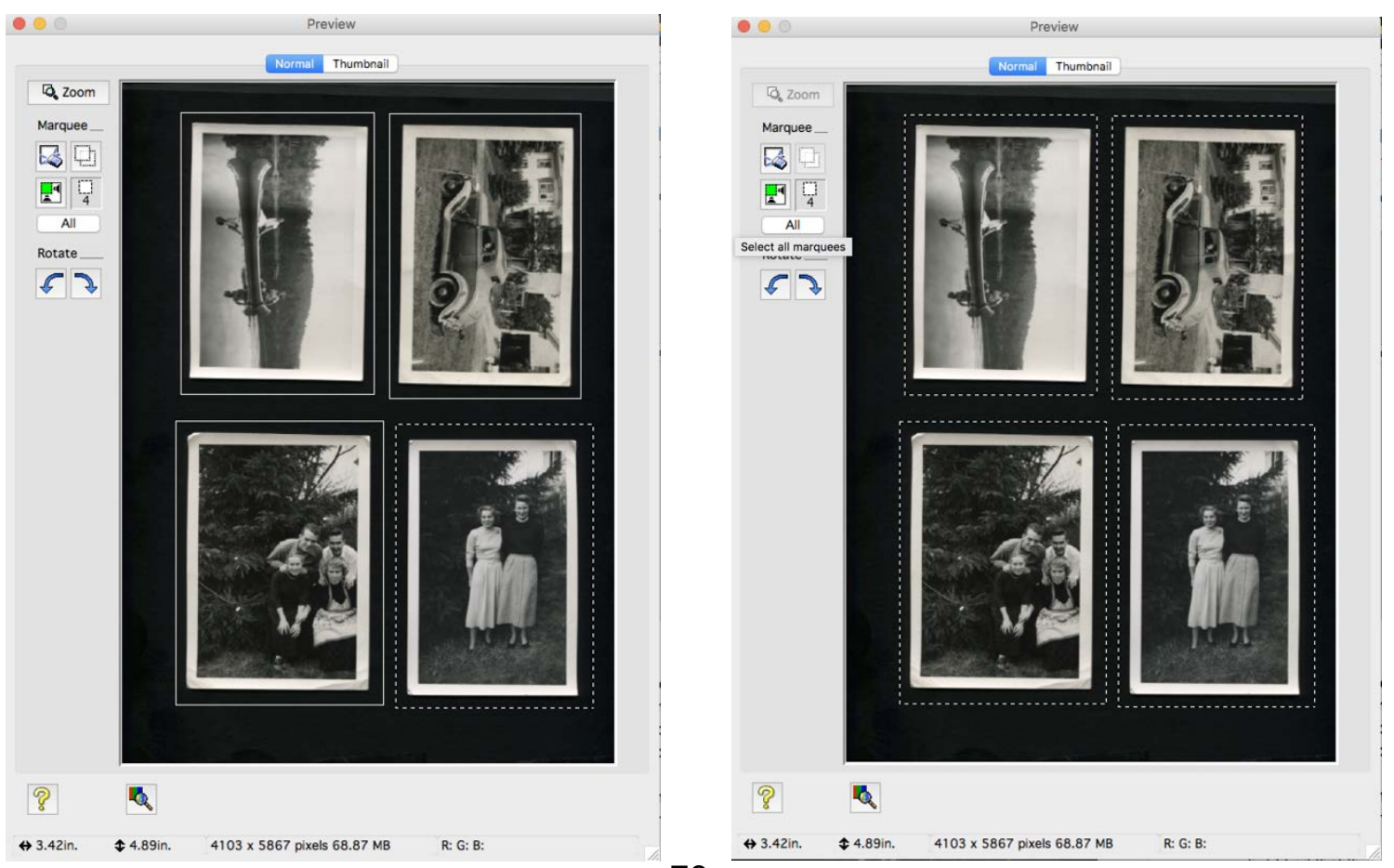
12. Make sure that Unsharp Mask, Descreening, Color Restoration, Backlight Correction, and Dust Removal are unchecked. FADGI guidelines recommend that all corrections should be done in post-production (i.e. Photoshop) on a copy of the original master scan. For more information about FADGI guidelines, refer to Section 4.1.

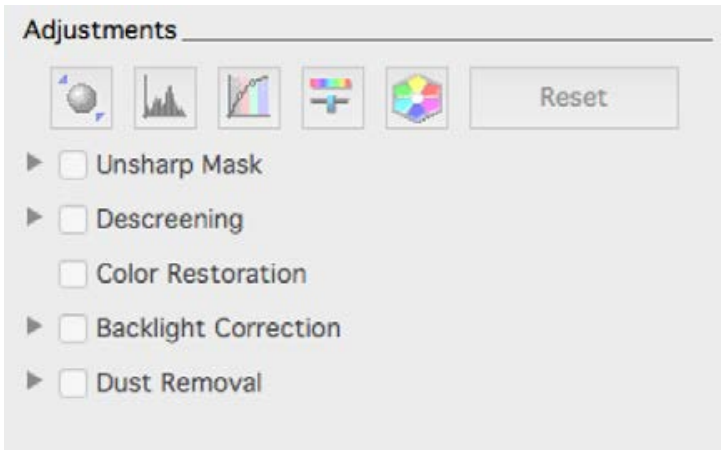

13. After you have drawn a marquee around the image(s) you want to scan, click SCAN.

14. In the dialog box called File Save Settings that now appears, select the LOCATION where you want your scanned images to automatically save, perhaps in a folder on your desktop or on an external hard drive.

Epson will automatically add a three-digit sequence number to the end of the scans, which is handy if you are scanning more than one item. For more information about file-naming, refer to Section 1.7 Creating a File-Naming Structure

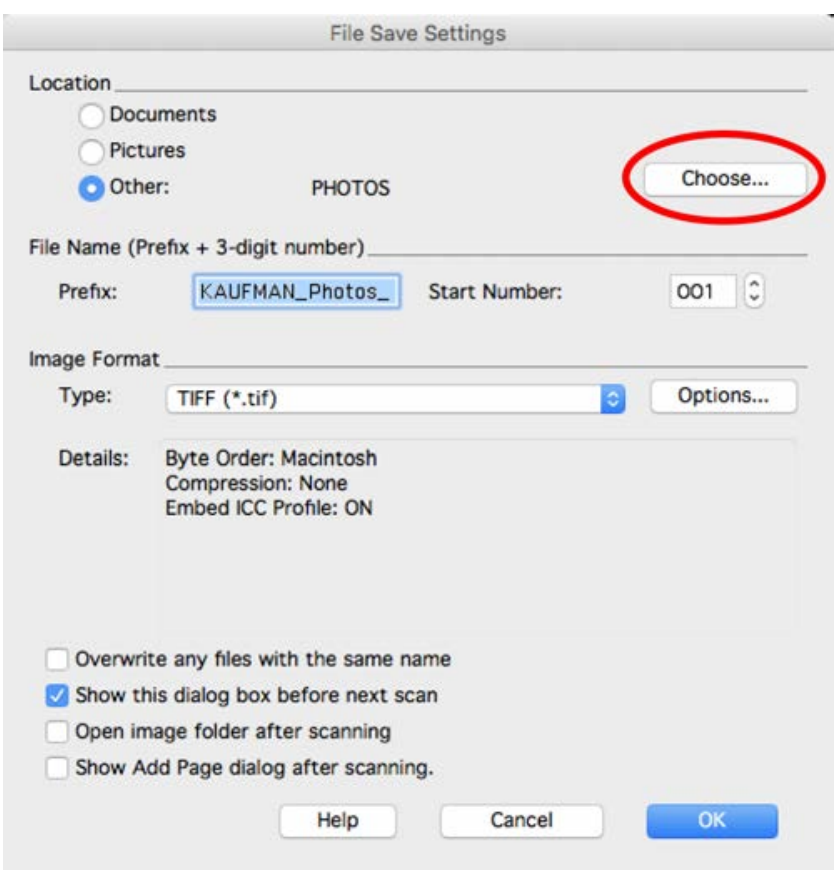


I am saving these scanned photographs in a PHOTOS folder (on my desktop which I will then copy to an external hard drive after the scanning session to back it up) within the parent KAUFMAN_ARCHIVING folder. You can be more specific in your location if you would like. For example, I could save these scans in a subfolder 'BW_Photos' or 'Photos_1945-1960' if I am scanning photographs from a specific date range.

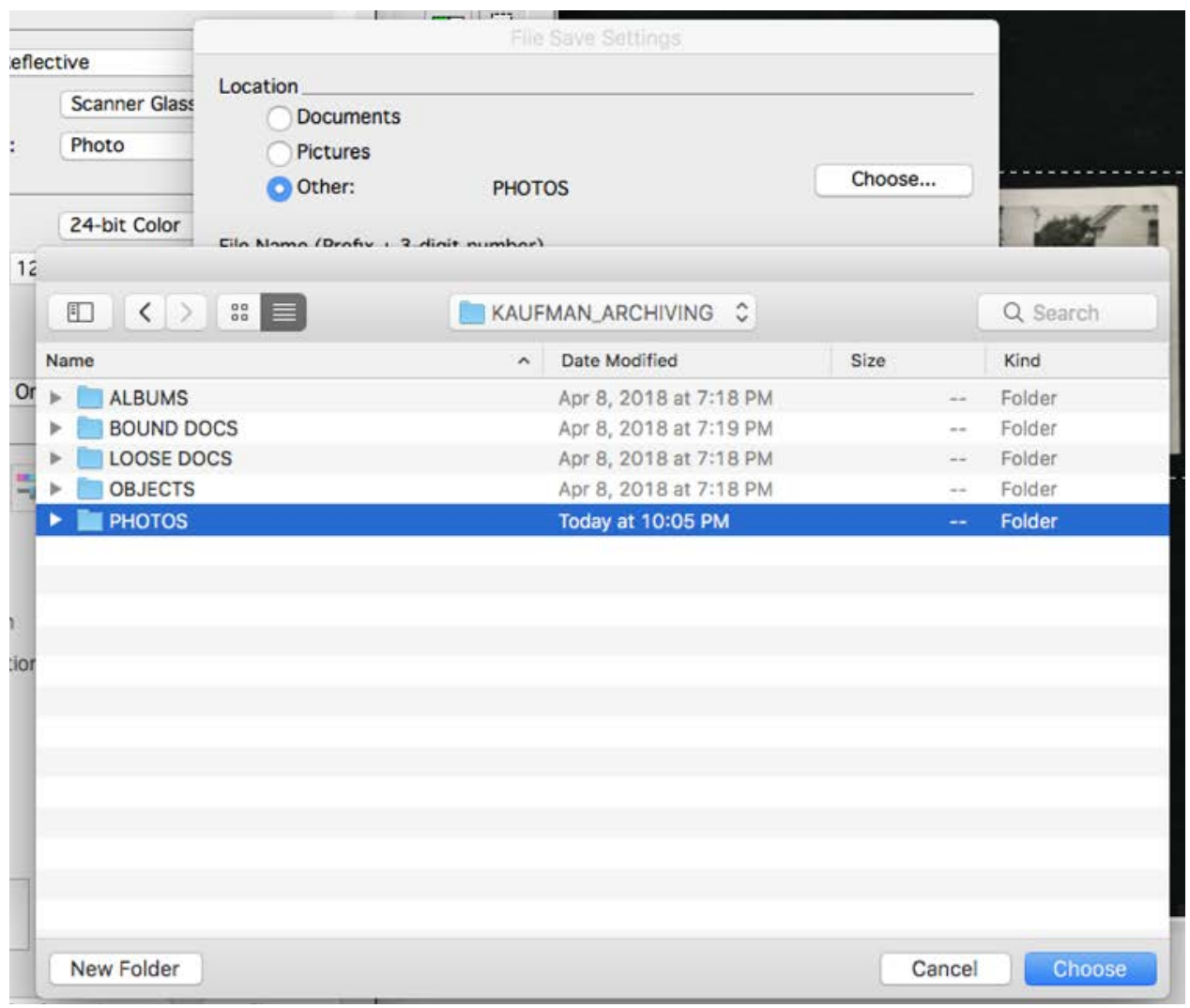

15. Click CHOOSE to select the folder.

16. Next type in your desired file name followed by the number at which you want the sequence to start. For more information about naming files, see section 1.8 Creating A File-Naming Structure.

17. Under IMAGE FORMAT > TYPE select TIFF ("tif). Then click OK and your scan should begin. While the image is scanning, the Epson interface will minimize but reappear once the scanning is complete. 
18. Now that you have scanned your image, you are done with the archival scanning. However, if you want to crop the image or make any adjustments to the exposure, tones, etc. refer to Section 3: Post-Processing.

\section{TIPS \& TRICKS}

If you are planning to scan a number of documents that are similar in size (but do not want to scan multiple images at once, you can place the document in a quadrant of the scanning glass, and then select that quadrant with the marquee tool. This way you will not need to do a preview scan for each document as long as it is placed in that preselected quadrant and your scanning project will go much more quickly. The image will scan with a much larger border, but you can crop this down in Photoshop or another image-editing program.

If you want to scan the front and back of a document, do not scan multiple documents at once as this will throw off the sequence numbering of the scans. You can go back and rename the files if you want to scan multiple fronts at once, and multiple backs at once, but I find that this can often get confusing and files can be mislabeled.

If you are using a Mac, there is a good chance that you will experience a slight glitch in the Epson Scan software: a scan will not begin, or the next scan in a sequence of scans, will not begin until you move the cursor. I have been having this very minor but very annoying issue for years and it does not appear that there will be any forthcoming fix from Epson anytime soon. 


\subsection{Scanning Slides}

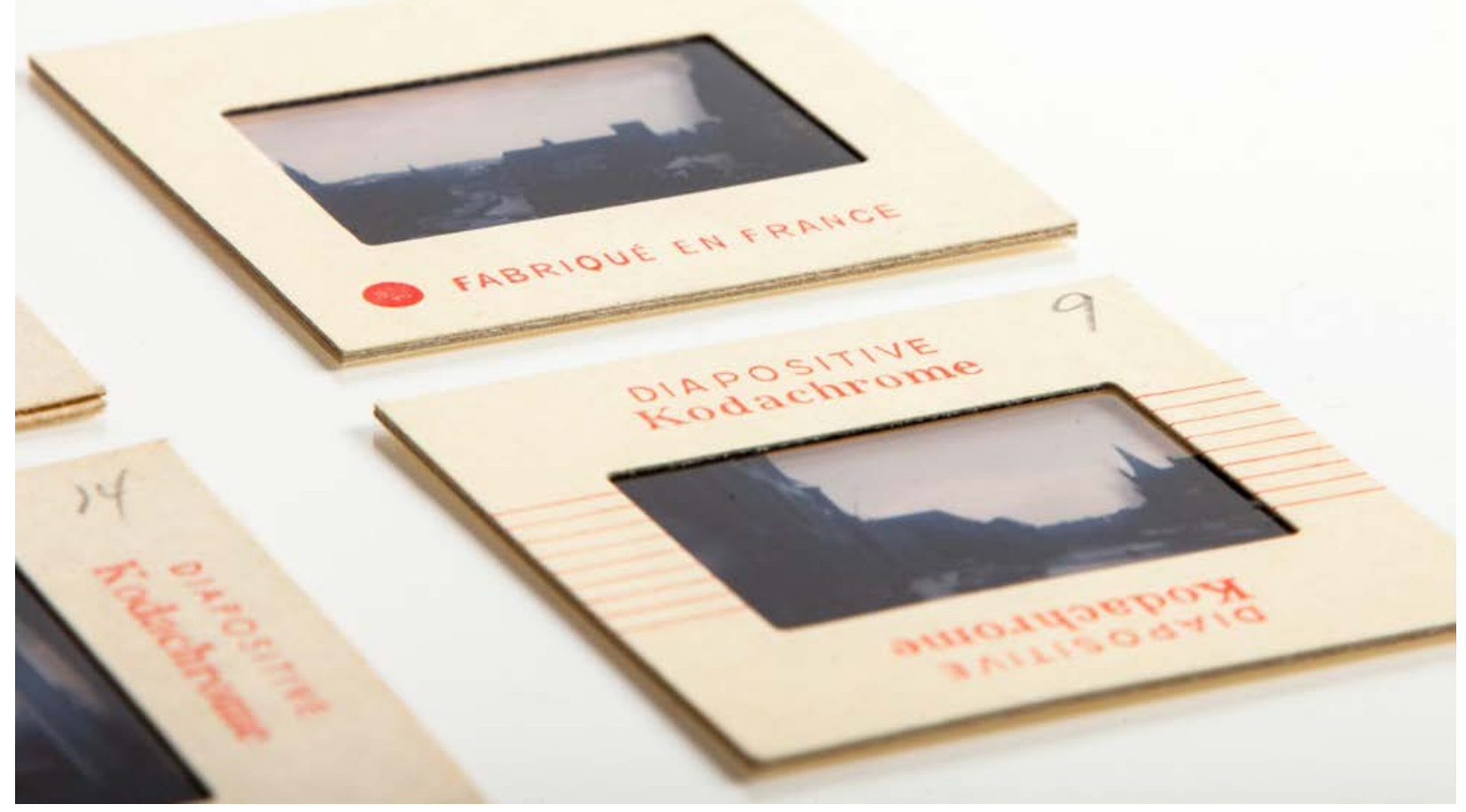

\section{What You Will Need:}

- Flatbed scanner (I used an Epson Perfection V370 Photo)

- EPSON Scan software, installed onto your computer

- Computer with Epson Scan software installed

- Slide holder accessory

- Cotton gloves

- Anti-static brush or microfiber cloth 
1. Plug connect the power cable and the USB cable provided.

2. Remove the document mat from the inside lid of the flatbed scanner.

3. Turn the scanner $\mathbf{O N}$.

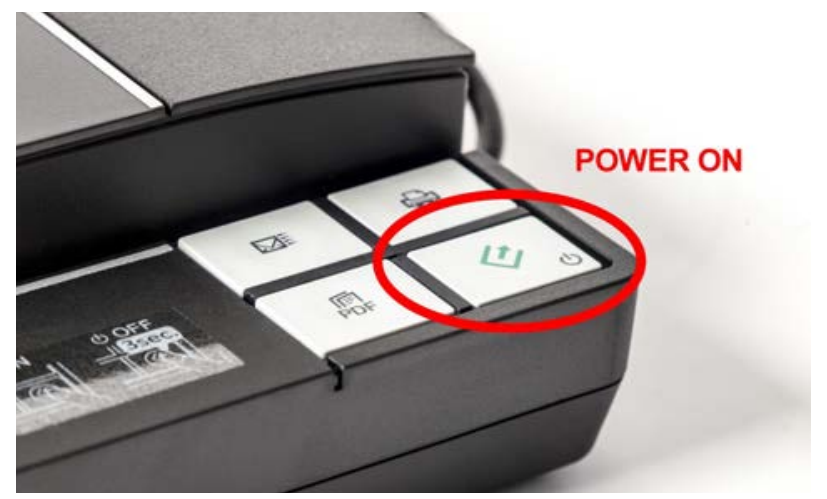

1. If you are just beginning to scan, lightly brush the scanner glass with an antistatic brush or microfiber cloth to remove any dirt and fingerprints.

4. Lay the scanning tray on the scanner glass, aligning the slide frames with the transparency window in the center of the scanner lid.

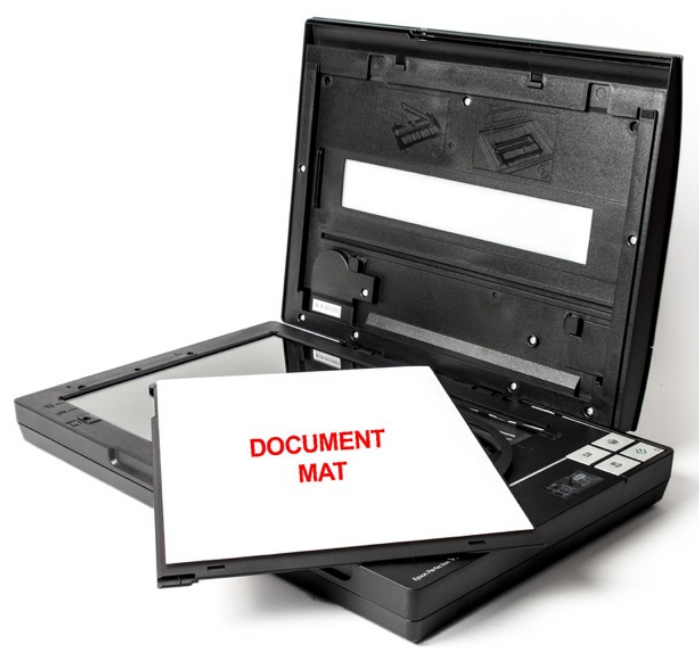

5. Match the guides on the tray to the corresponding markings along the side of the scanner glass.

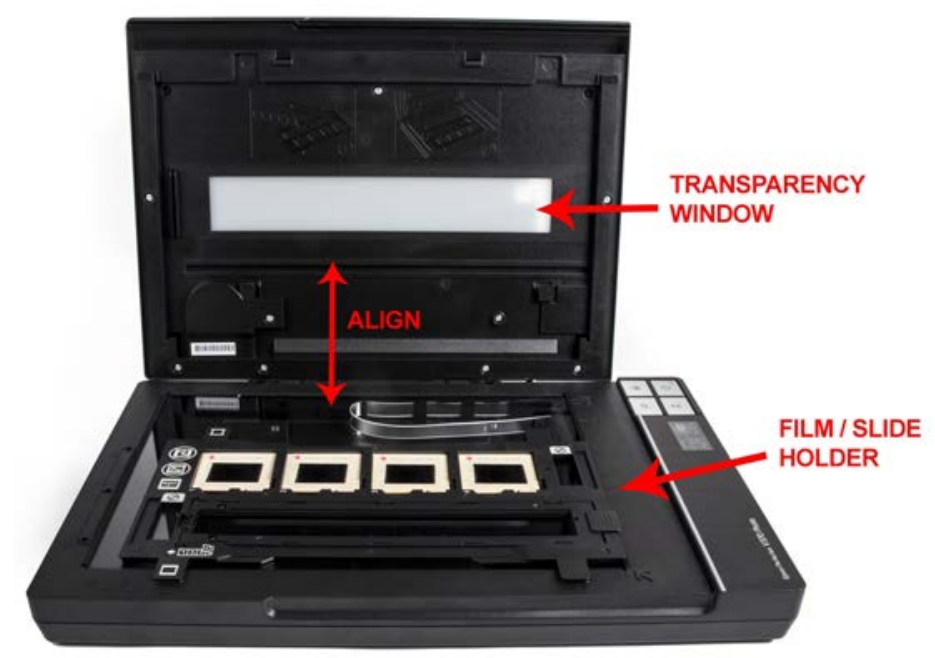


6. Place the slides in the holder EMULSION SIDE UP (shiny side down), following the diagram on the side of the tray.

The emulsion side of a negative or slide is the side that is coated with photosensitive chemicals. It usually has a less-reflective, duller quality than the reverse side when angled towards a light, and may sometimes even have a visible texture. Depending on the type of film, the emulsion side may also show a slight relief of the image. It can be very difficult to tell which side is the emulsion side, especially with slides. If there are any signs or writing in the image, the letters will be backwards on the emulsion side. If you are still unable to tell which side is the emulsion side, you can try scanning both ways and then either see which is sharper (this is an indication of the correct orientation). As a rule of thumb, the emulsion side should always face the light source of the scanner because this prevents any kind of reflection of refraction that may occur when scanning through the plastic base first. The bright strip of light that you see

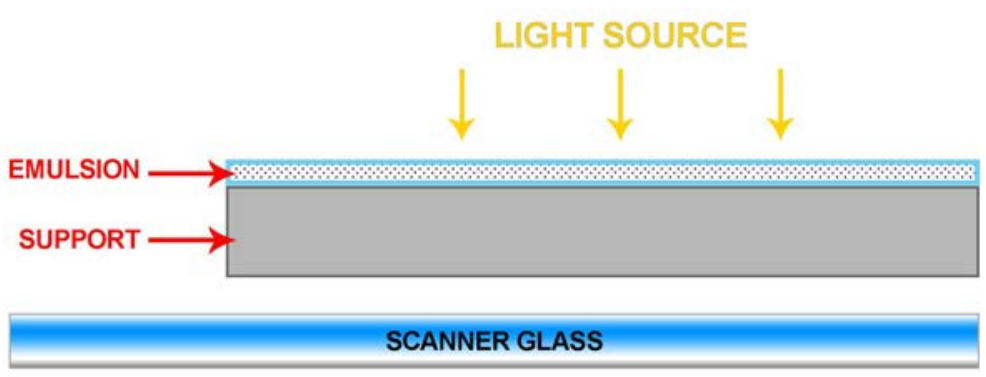
moving across the scanning bed under the glass is not the light source, it is the digital sensor that digitizes the image. The strip in the inside center of the lid is the light that shines through the film, allowing the scanner to see it. If you were to try to scan slides or film with the document mat still attached to the inside of the scanner lid, covering the light window in the lid, the scans would appear totally black.

7. Close the scanner lid.

EPSON Scan

8. Open the EPSON Scan program.

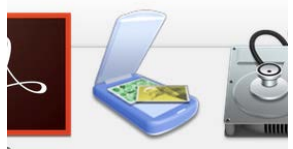




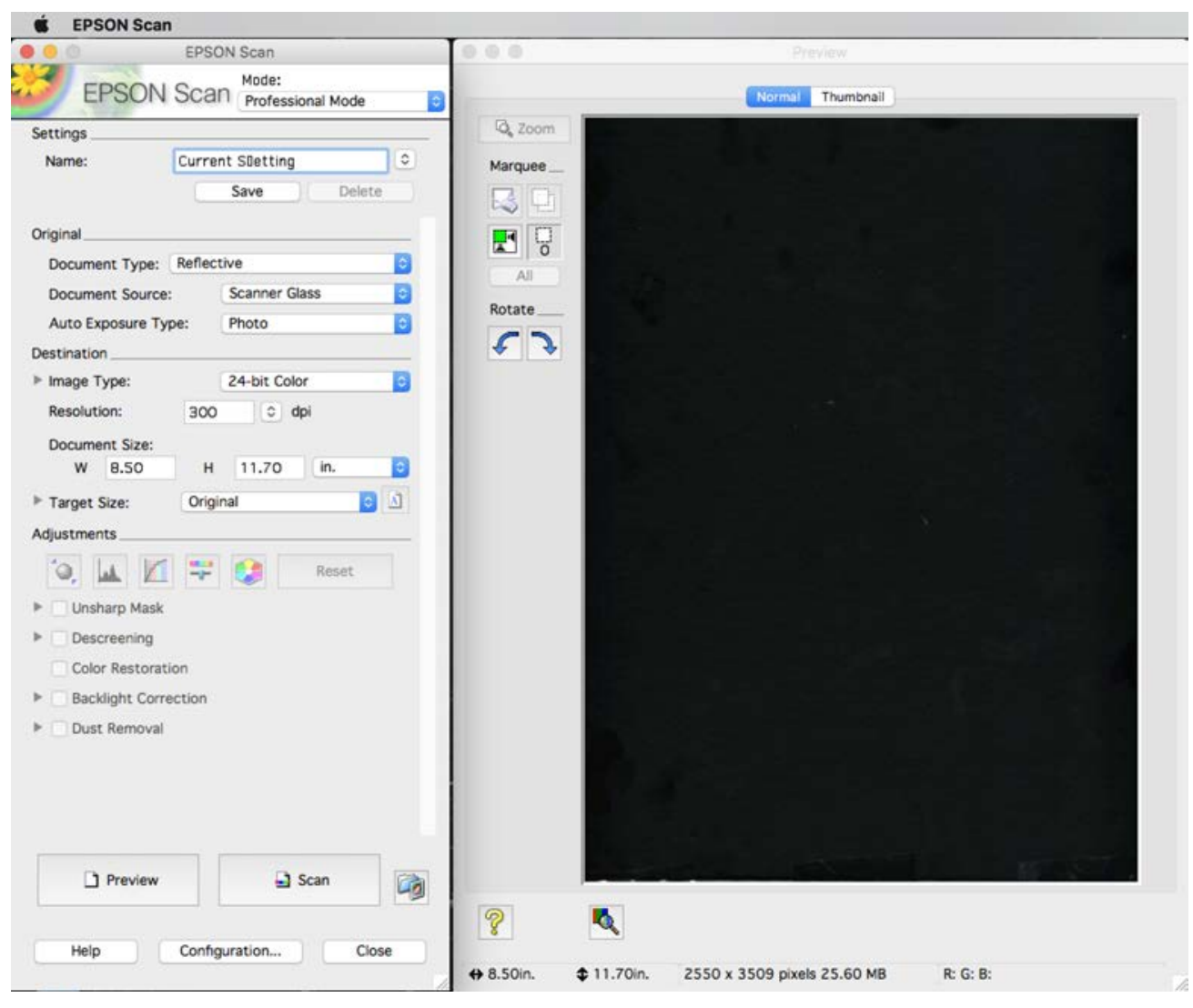

EPSON Scan interface

9. Set scan mode to PROFESSIONAL.

10. Set Document Type to FILM.

11. Set Film Type to POSITIVE FILM.

12. Set Image Type to 48-bit Color.

\section{Click PREVIEW.}

14. Four preview thumbnails will now be displayed. To rotate or flip the thumbnails, click either of the icons under Frame.

EPSON Scan

\begin{tabular}{|c|c|c|c|}
\hline \multicolumn{4}{|c|}{$\begin{array}{l}\text { Mode: } \\
\text { Professional Mode }\end{array}$} \\
\hline \multicolumn{4}{|l|}{ Settings } \\
\hline \multirow[t]{2}{*}{ Name: } & Current Setting & & $\hat{\imath}$ \\
\hline & Save & Delet & \\
\hline \multicolumn{4}{|l|}{ Original } \\
\hline Document Type: & Film & & 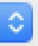 \\
\hline Film Type: & Positive Film & & $\hat{\theta}$ \\
\hline \multicolumn{4}{|l|}{ Destination } \\
\hline Image Type: & 48-bit Co & & $\hat{0}$ \\
\hline
\end{tabular}

\section{Frame \\ 監蕅}




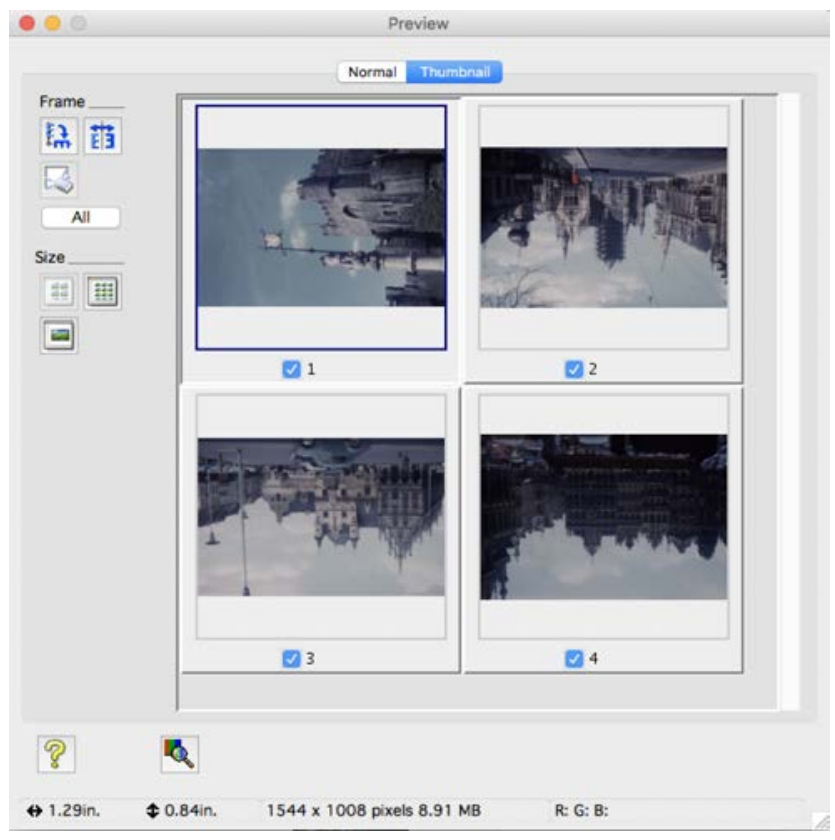

Original thumbnails

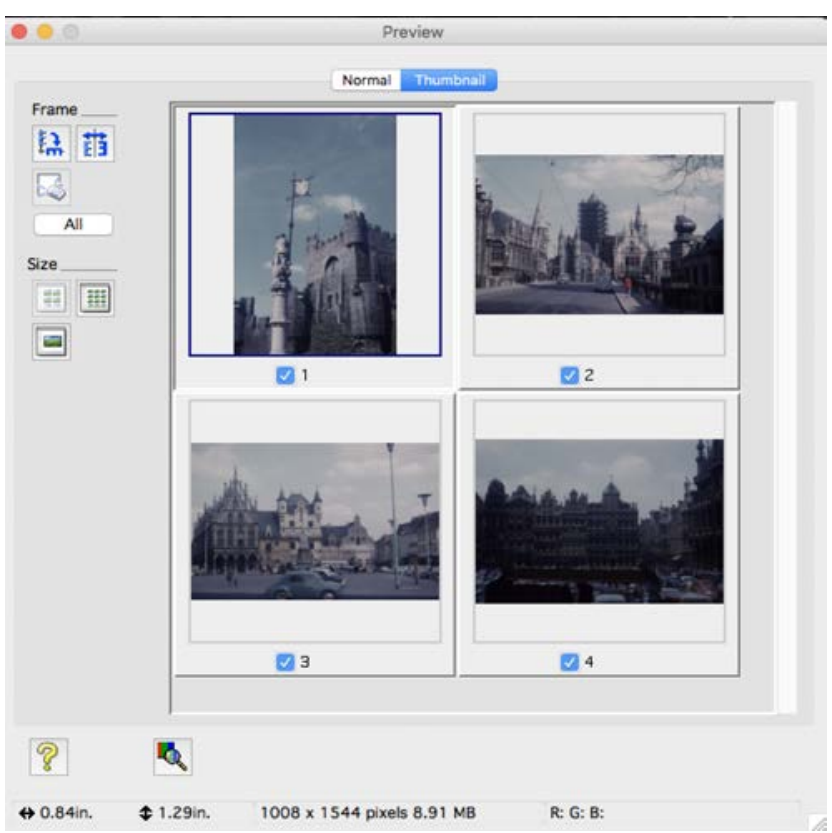

Rotated thumbnails

You can display the slide thumbnails in either NORMAL view or THUMBNAIL view. Choose the normal view if you want to include a border in your images by using the marquee selection tool to manually draw a marquee around the area you wish to scan. I will be using this method so as to be consistent with my other digitized files.
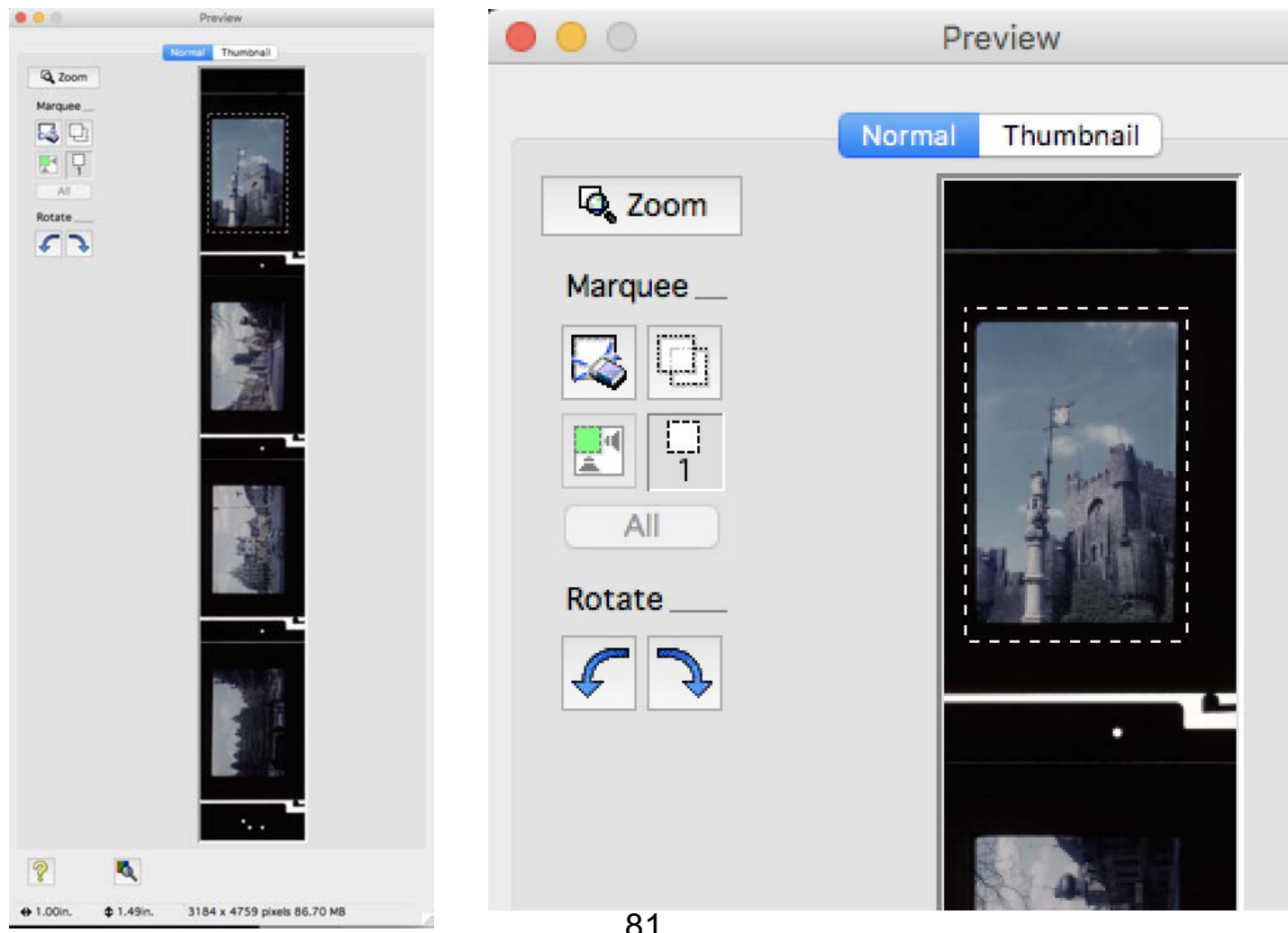
15. To set the resolution, select one of the thumbnails and look at the bottom of the Preview window to see the resolution of the scan. You will see the width and height in both inches and pixels, as well as the size of the file if scanned at the resolution currently set, in this case $1200 \mathrm{dpi}$.
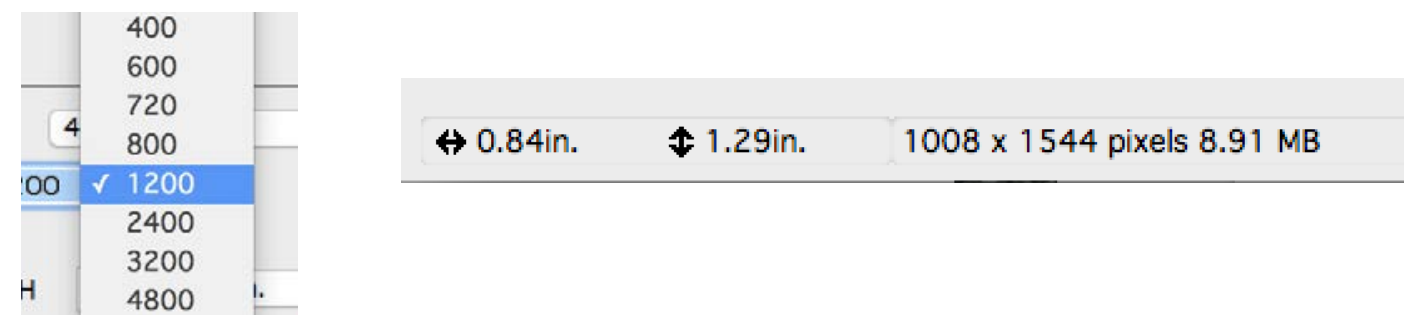

Keeping in mind the FADGI recommendation of 4,000 to 5,000 pixels on the long side, click through the resolution options in the drop down menu and see which value increases the dpi to between 4,000 and 5,000 pixels.

I found that $3200 \mathrm{dpi}$ is a sufficient resolution at which to scan these slides because at that resolution the long edge is over 4,000 pixels. If I wanted the dimensions to sit more comfortable between the 4,000 and 5,000 pixel size range, I could also manually input a dpi of around 4000 .

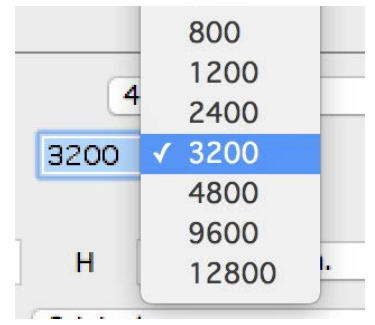

16. Make sure that Unsharp Mask, Descreening, Colour Restoration, Backlight Correction, and Dust Removal are unchecked.

\section{$\leftrightarrow$ 0.84in. 1 1.29in. $2688 \times 4117$ pixels $63.32 \mathrm{MB}$}


17. Select the thumbnails you want to scan by checking the boxes under each or if scanning in the NORMAL view mode, use the marquee tool to draw boxes around each image and then click ALL. If only one marquee box is active (appearing as a dashed line) the scanner will only scan that slide.

When switching between the two, you will see a notification warning that the settings you set after generating the preview will be reset, meaning you will have to make the adjustments again in the new preview mode. If you do not mind this, click OK. Otherwise remain in the current Preview mode.

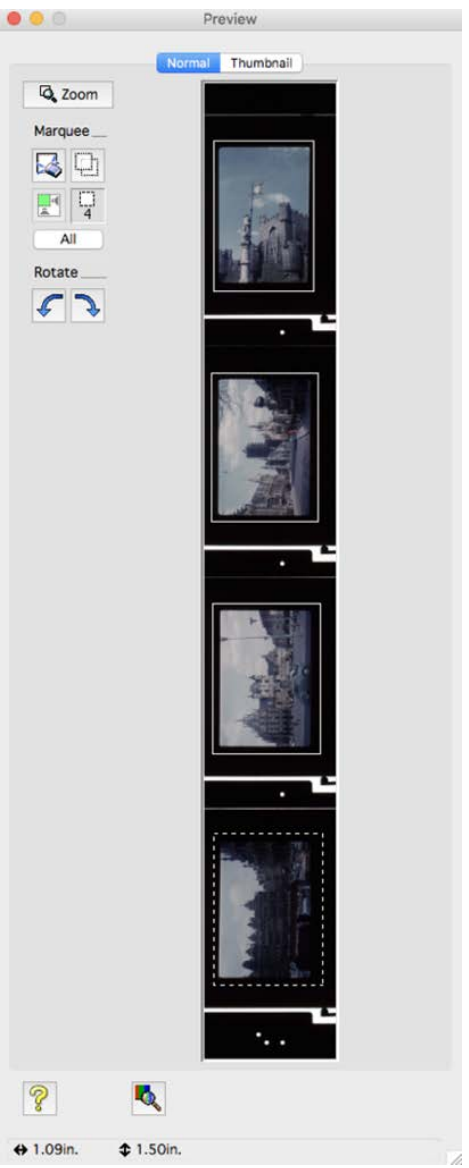

Single active marquee box
Some of the settings that you change after preview will be initialized if you change the Preview mode. $\mathrm{OK}$ to continue?

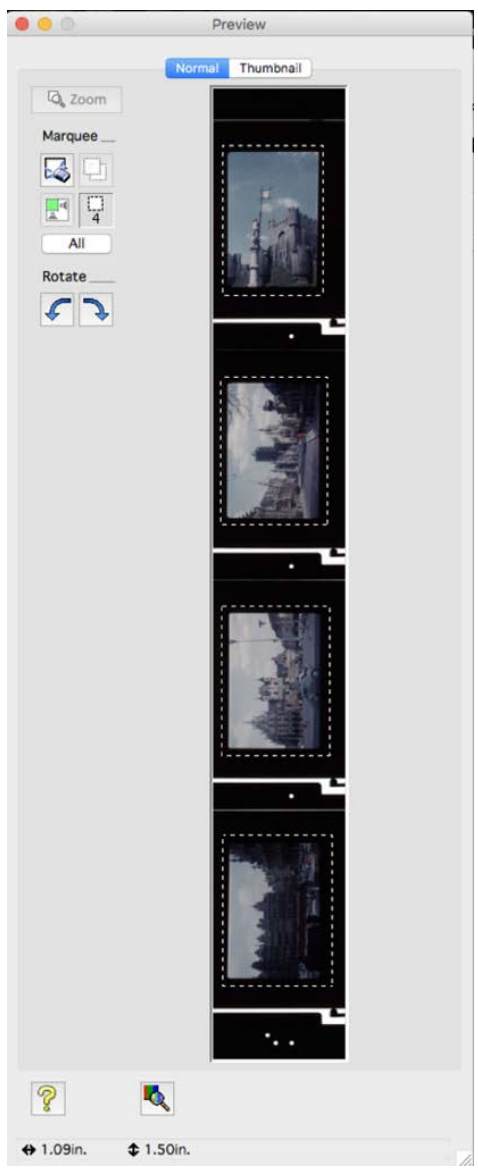

ALL active marquee boxes 
18. Click SCAN.

19. In the dialog box called File Save Settings that now appears, select the LOCATION where you want your scanned images to automatically save.

\section{S Scan}

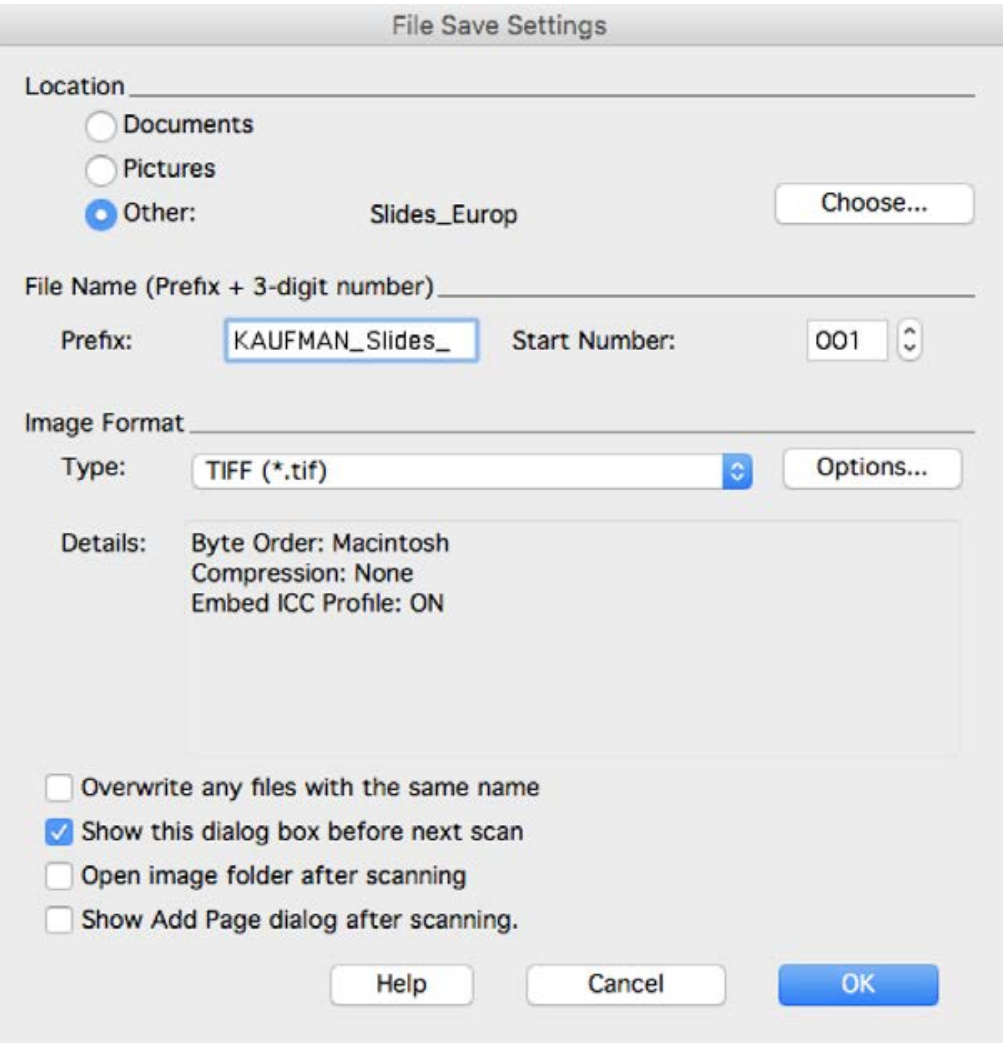

I am saving these scanned slides in a folder called 'Slides_Europe_1958' within the PHOTOS folder.

\begin{tabular}{|c|c|c|c|c|c|c|c|}
\hline 目 & $<$ & $>$ & $\therefore \equiv$ & \multicolumn{2}{|c|}{ PHOTOS } & $\hat{\imath}$ & Q Search \\
\hline \multicolumn{4}{|l|}{ Name } & $\hat{\wedge}$ & Date Modified & Size & Kind \\
\hline \multicolumn{4}{|c|}{ DW_Photos_1940s-1960s } & \multicolumn{3}{|c|}{ Apr 3, 2018 at $9: 37 \mathrm{PM}$} & Folder \\
\hline \multicolumn{4}{|c|}{ Slides_Europe_1958 } & \multicolumn{3}{|c|}{ Today at 12:15 PM } & Folder \\
\hline
\end{tabular}


Here is the folder and file structure:

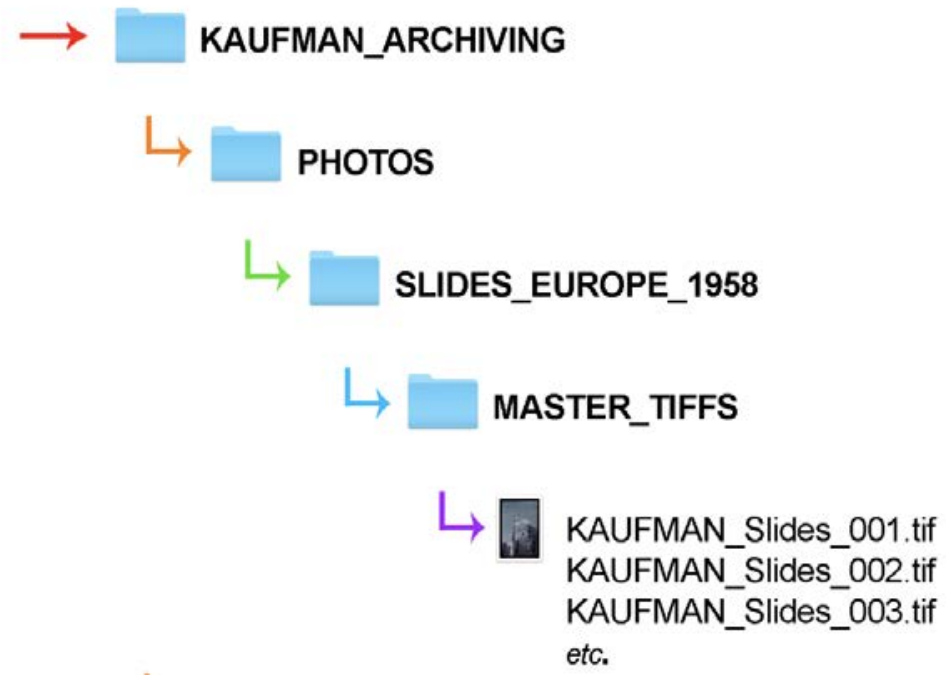

\section{Click CHOOSE.}

21. Next type in your desired file name followed by the number at which you want the sequence to start. For more information about naming files, see section 1.8 Creating A File-Naming Structure.

22. Under IMAGE FORMAT $\rightarrow$ TYPE select TIFF ( ${ }^{*}$ tif). Then click OK and your scan should begin.

23. Once you have finished scanning your images, continue to Section 3: PostProcessing. 


\subsection{Scanning Film}

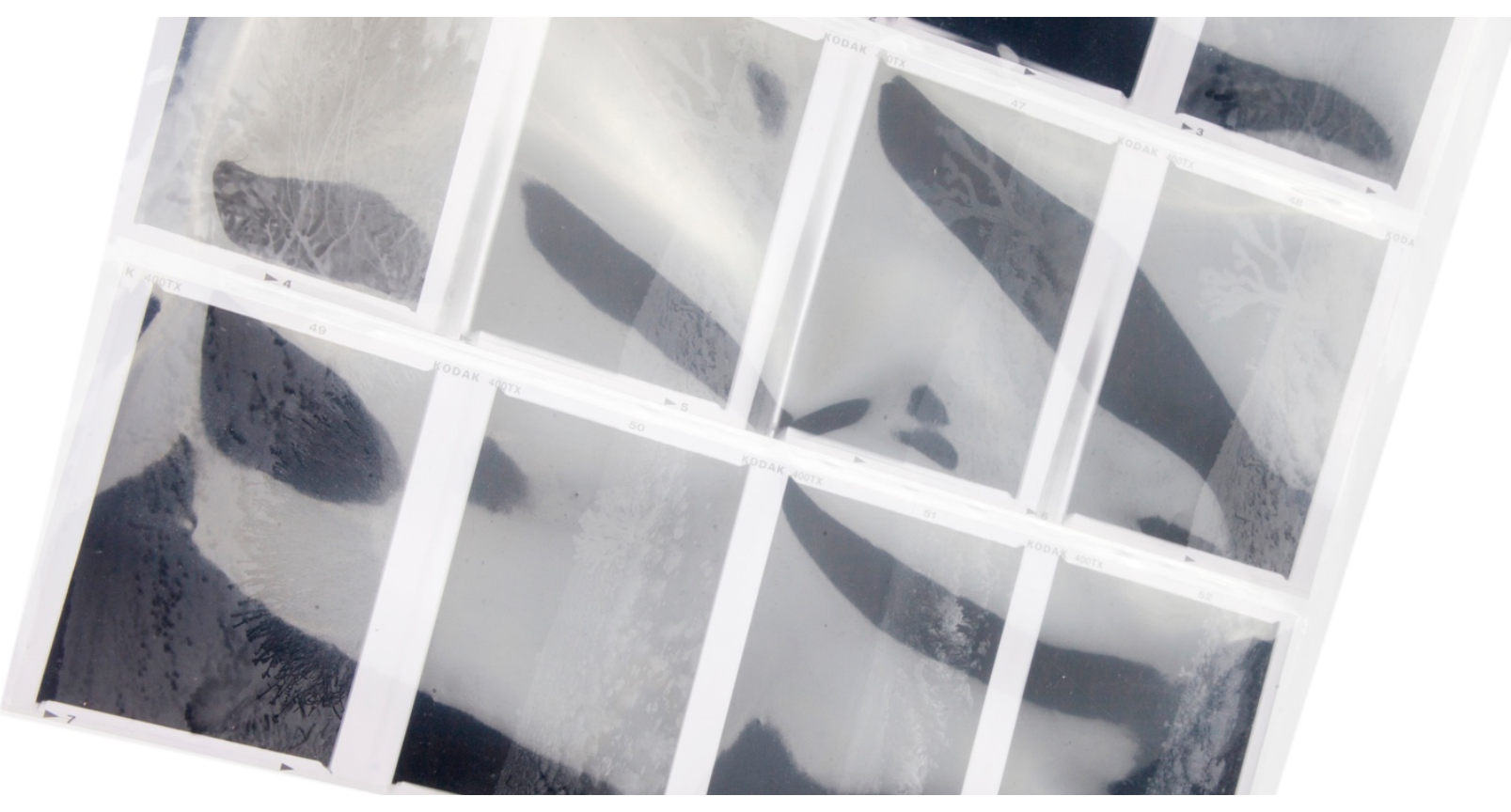

\section{What You Will Need:}

- Flatbed scanner (I used an Epson Perfection V370 Photo)

- EPSON Scan software, installed onto your computer

- Computer with Epson Scan software installed

- Slide holder accessory

- Cotton gloves

- Anti-static brush 
1. Plug connect the power cable and the USB cable provided.

2. Remove the document mat from the inside lid of the flatbed scanner.

3. Turn the scanner $\mathbf{O N}$.

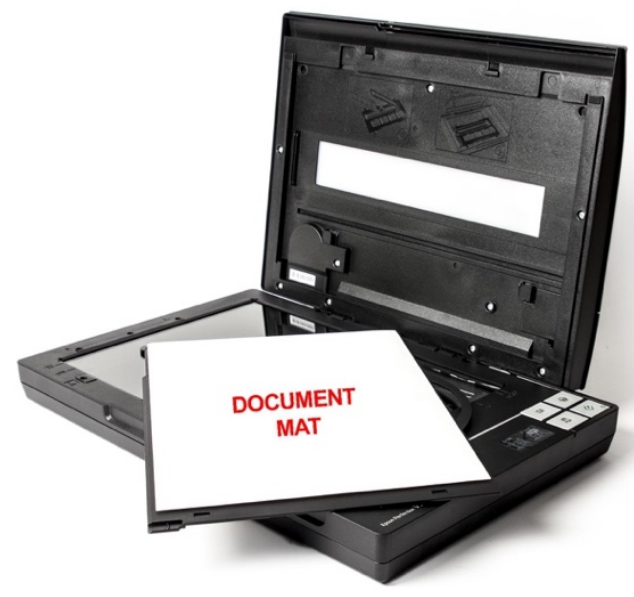

4. If you are just beginning to scan, lightly brush the scanner glass with an antistatic brush or microfiber cloth to remove any dirt and fingerprints.

5. Lay the scanning tray on the scanner glass, aligning the film frame with the transparency window in the center of

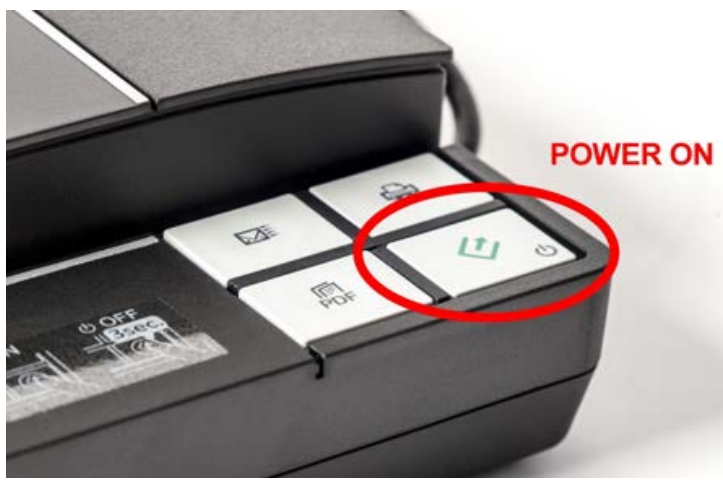
the scanner lid.

6. Lift the film strip cover using the tap on the left side.

7. While wearing gloves, hold the film strip by the edges, being careful not to touch the film surface and gently slide it EMULSION-SIDE UP into the two tabs at the right end of the film holder.

8. Lower the film strip cover over the film and snap into place.

9. Close the scanner lid.

EPSON Scan

10. Open the EPSON Scan program. 


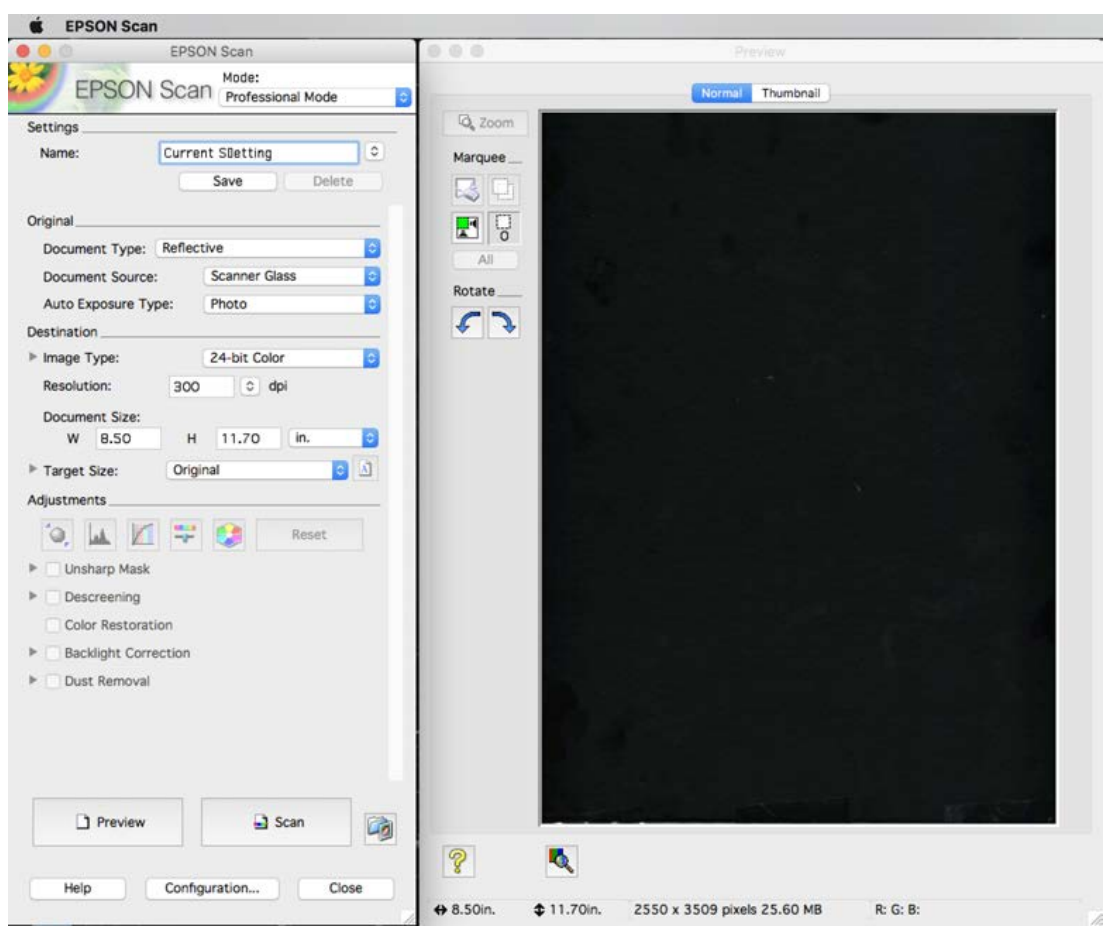

11. Set scan mode to PROFESSIONAL.

12. Set Document Type to FILM.

13. Set Film Type to POSITIVE FILM. ${ }^{36}$

14. Set Image Type to 16-bit Grayscale.

If you are scanning colour film, set Film

Type to Color Negative Film and the Image

Type to 48-bit Color.

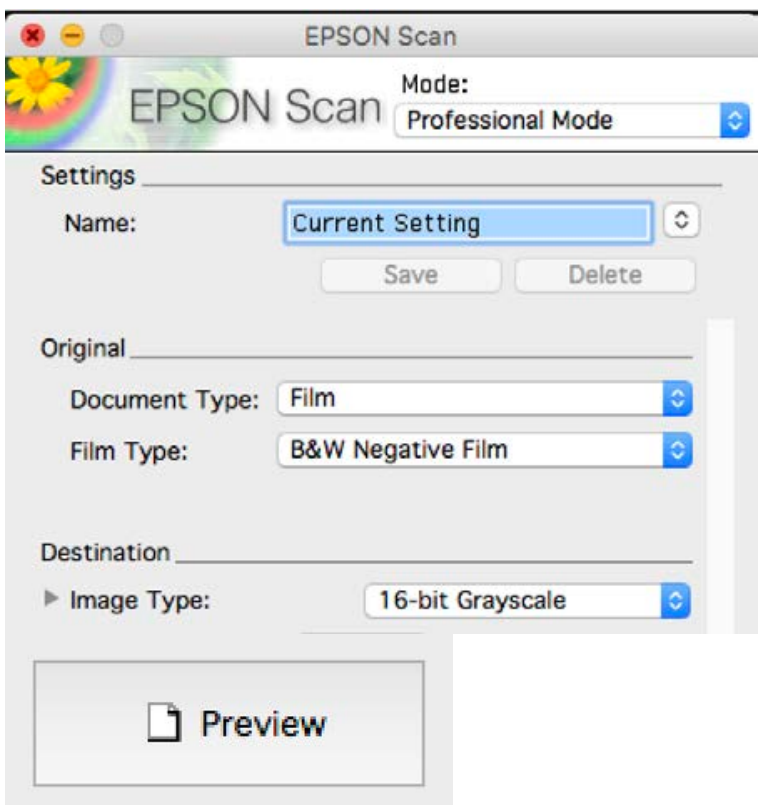

\section{Click PREVIEW.}

16. Six preview thumbnails (or however many frames are on the strip of negatives) will now be displayed. To rotate or flip the thumbnails, click either of the icons under Frame.

36. FADGI guidelines recommend that black and white negatives be scanned as positives so the final TIFF files will be negatives as well. The images can then be inverted in post-production. In Photoshop, simply press COMMAND + $\mathrm{i}$ on a Mac or CONTROL $+\mathrm{i}$ on a PC. 
You can display the negative strip in either NORMAL view (in which the entire strip is visible) or THUMBNAIL view. Choose the normal view if you want to include a border in your images by using the marquee selection tool to manually draw a marquee around the area you wish to scan. I will be using this method so as to be consistent with my other digitized files.

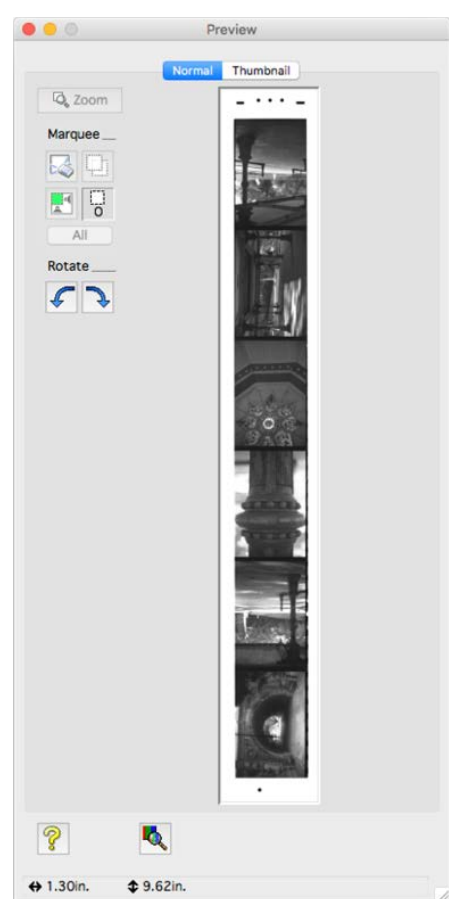

Normal View

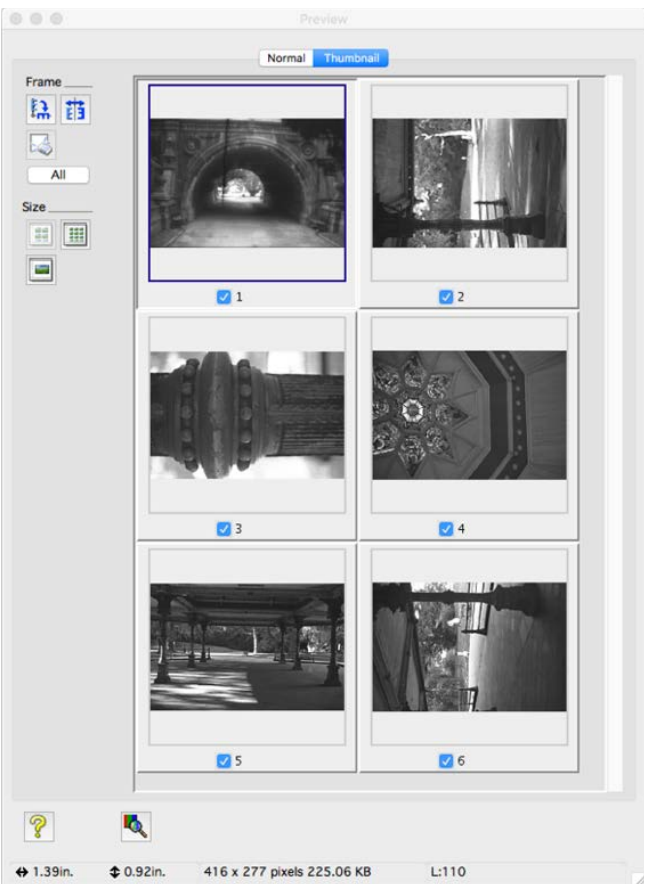

Thumbnail View

When switching between the two, you will see a notification warning that the settings you set after Some of the settings that you change after preview will be initialized if you change the Preview mode. generating the preview will be reset, meaning you will have to make the adjustments again in the new preview mode. If you do not mind this, click OK. Cancel Otherwise remain in the current Preview mode.

17. To set the resolution, select one of the thumbnails or draw a selection box around one of the images on the strip of film, and look at the bottom of the Preview window to see what the resolution of the scan will be at the current settings. You 
will see the width and height in both inches and pixels, as well as the size of the file if scanned at the resolution currently set, in this case $1200 \mathrm{dpi}$.
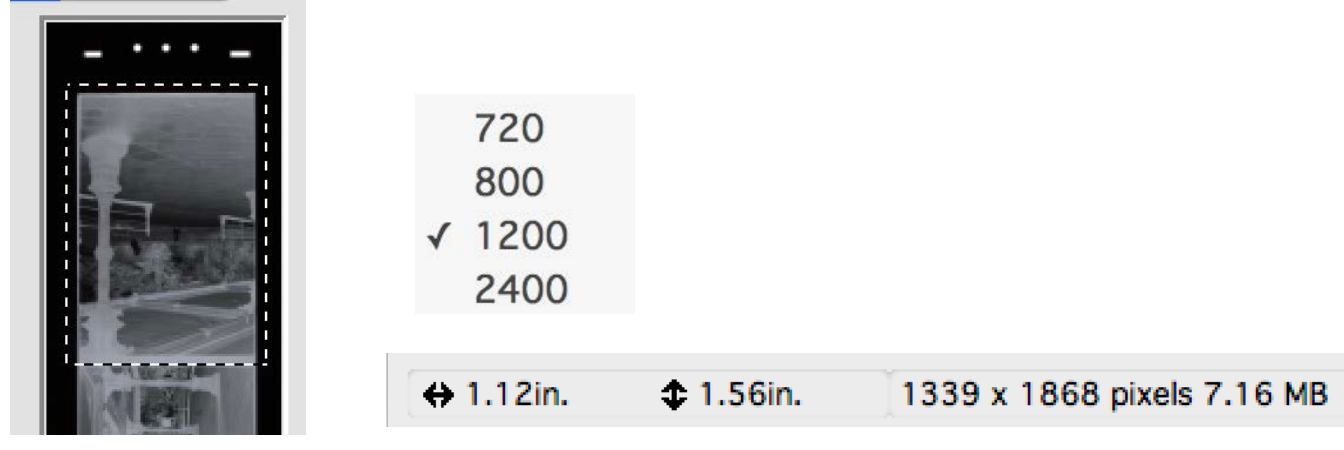

As you can see, the dimensions are 1339 × 1886 pixels. The long edge, currently 1868 pixels, should be between 4,000 and 5,000 pixels, so I will adjust the resolution in the drop-down menu. After trying different resolutions I can see that scanning at $3200 \mathrm{dpi}$ will provide an adequate pixel dimension. Note, you do not need to re-preview the scan to see the change in pixel dimension. Epson will project what the dimensions will be when you scan at the settings you have selected.
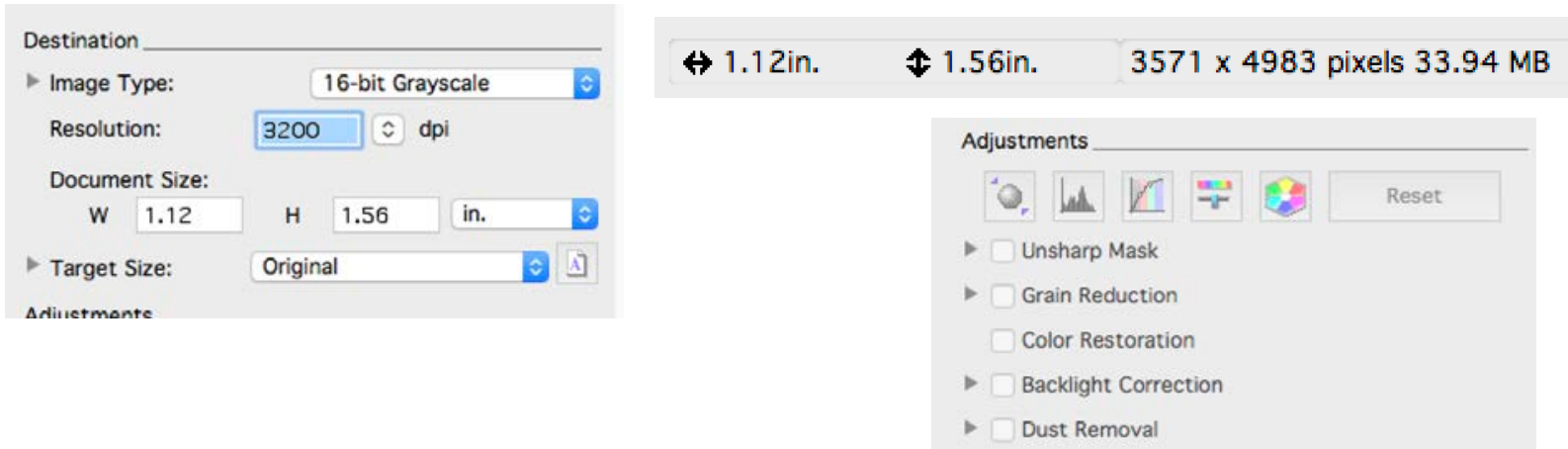

18. Make sure that none of the boxes in the Adjustment section are selected.

19. Click SCAN.

20. The File Save Settings window will now

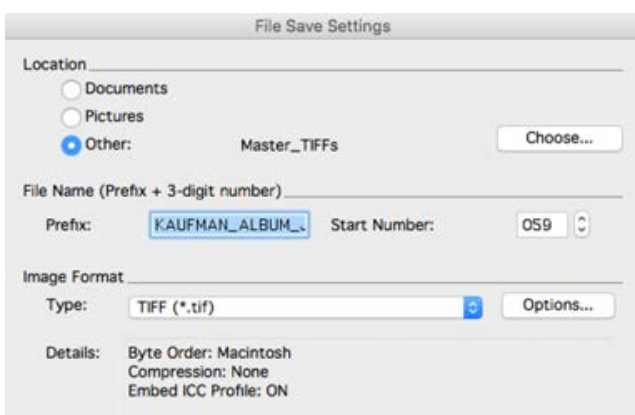
appear. 
21. Click CHOOSE to select the folder where all of the scans will automatically be saved.

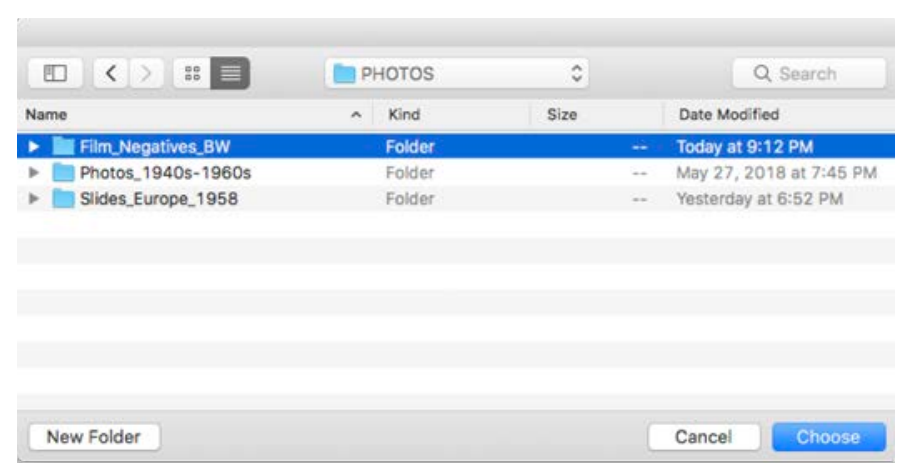

To create a new folder, click NEW FOLDER, type the name of the folder, then click CREATE.

Name of new folder:

MASTER_TIFFS

22. Once you have selected the folder, click CHOOSE.

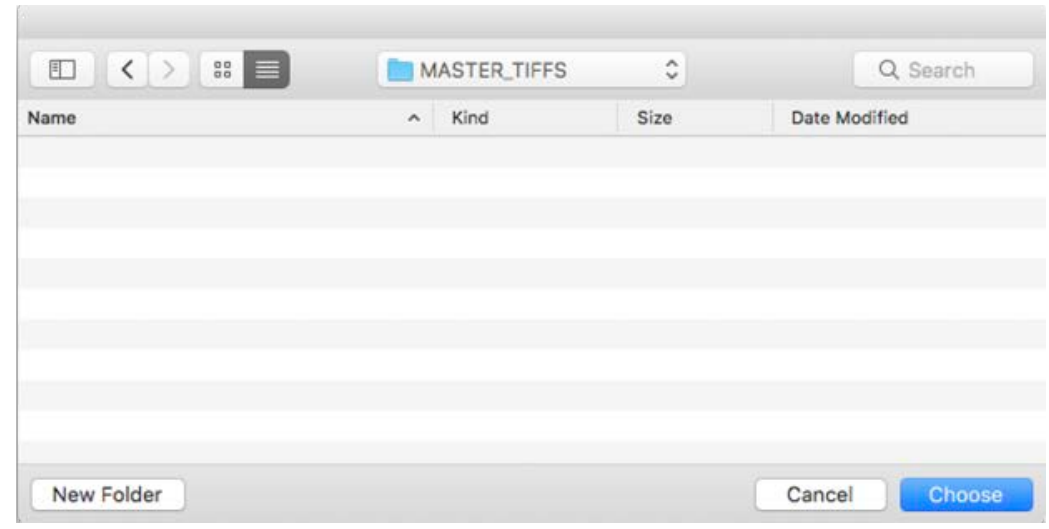

23. Next type in your desired file name prefix, then click OK. For information about file-naming, refer to section 1.8 Creating a File-Naming Structure. 


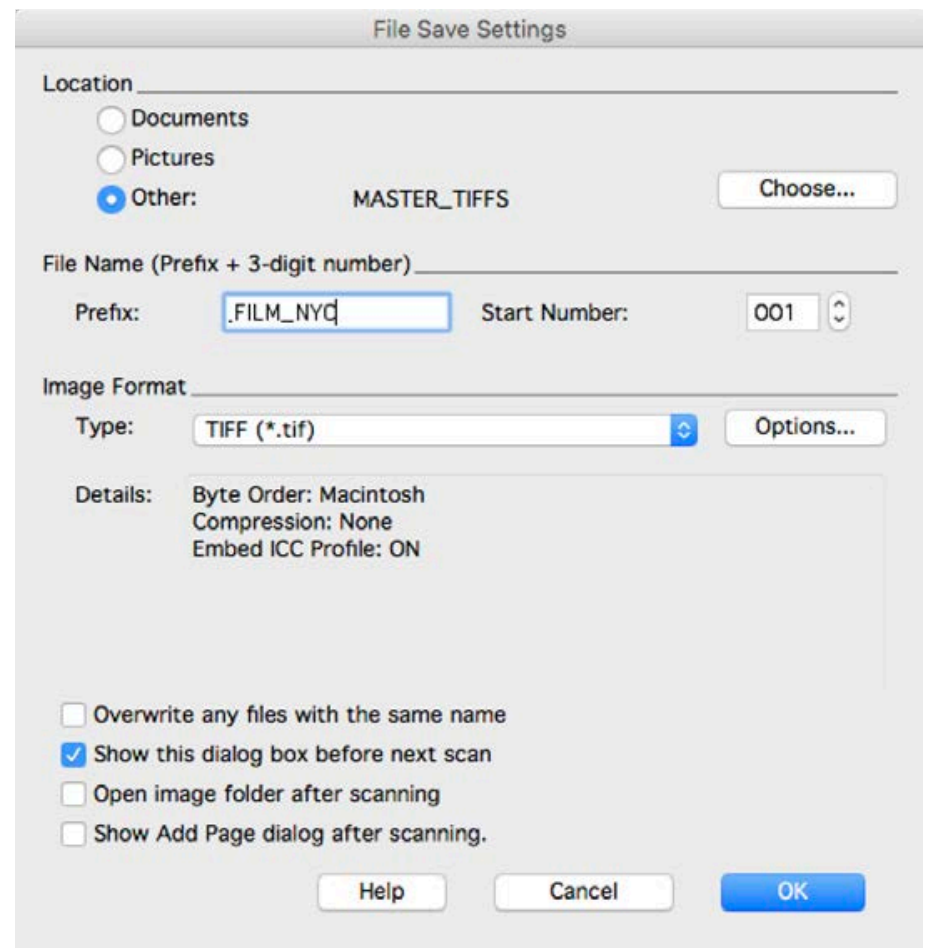

\section{Under IMAGE FORMAT > TYPE select}

TIFF (*tif). Then click OK and your scan

should begin.

25. Once you have finished scanning your images, continue to Section 3: PostProduction 


\subsection{Scanning and Compositing Oversized Documents}

You may have documents that are too big to fit in your flatbed scanner. Whether they are maps, blueprints, drawings, albums, or books, there are a number of alternative ways you can scan a large document. If you have a comfortable knowledge of Photoshop or other image editing software, you can scan the document in sections and then composite them together into a full image. However, this is not advisable for fragile documents as this method requires a lot of handling. Another option is to use a digital camera to photograph each page using either natural or artificial light, although this method can be finicky depending on the size of the document, its surface texture or shine, and the photographic equipment you have. The third option I will explore is photographing the documents with the HoverCam Solo 8Plus, a relatively reasonablypriced (as far as digital optical equipment goes) camera scanner that is portable, userfriendly, and-wonderfully for archivists-can capture images as TIFFs. I am in no way affiliated with Hovercam, but this seems to be one of the few document scanners available today (2018) that is both budget-friendly and can produce archival files.

I tested these methods using a photo album of my mother's trip to Peru in the 1970s. The album is very sentimental, but unfortunately the pages are the very common sticky magnetic type that are terrible for the photographs affixed to them. As you can see in the album, the pages have yellowed significantly, the pages have become tacky as the adhesive ages and dries, and these symptoms will only get worse with time until the album is a sticky yellow mess and the photographs irreparably damaged. Often photographs have fused so thoroughly that they are very difficult, if not impossible, to remove without causing physical damage. My goal is to digitize the album as it currently is, remove the photographs and place them on new, archival acid free pages, and replace those pages back into the original album binding, with interleaving tissue between each page. It may be best to seek professional help with documents like these, although that can become costly very quickly. 

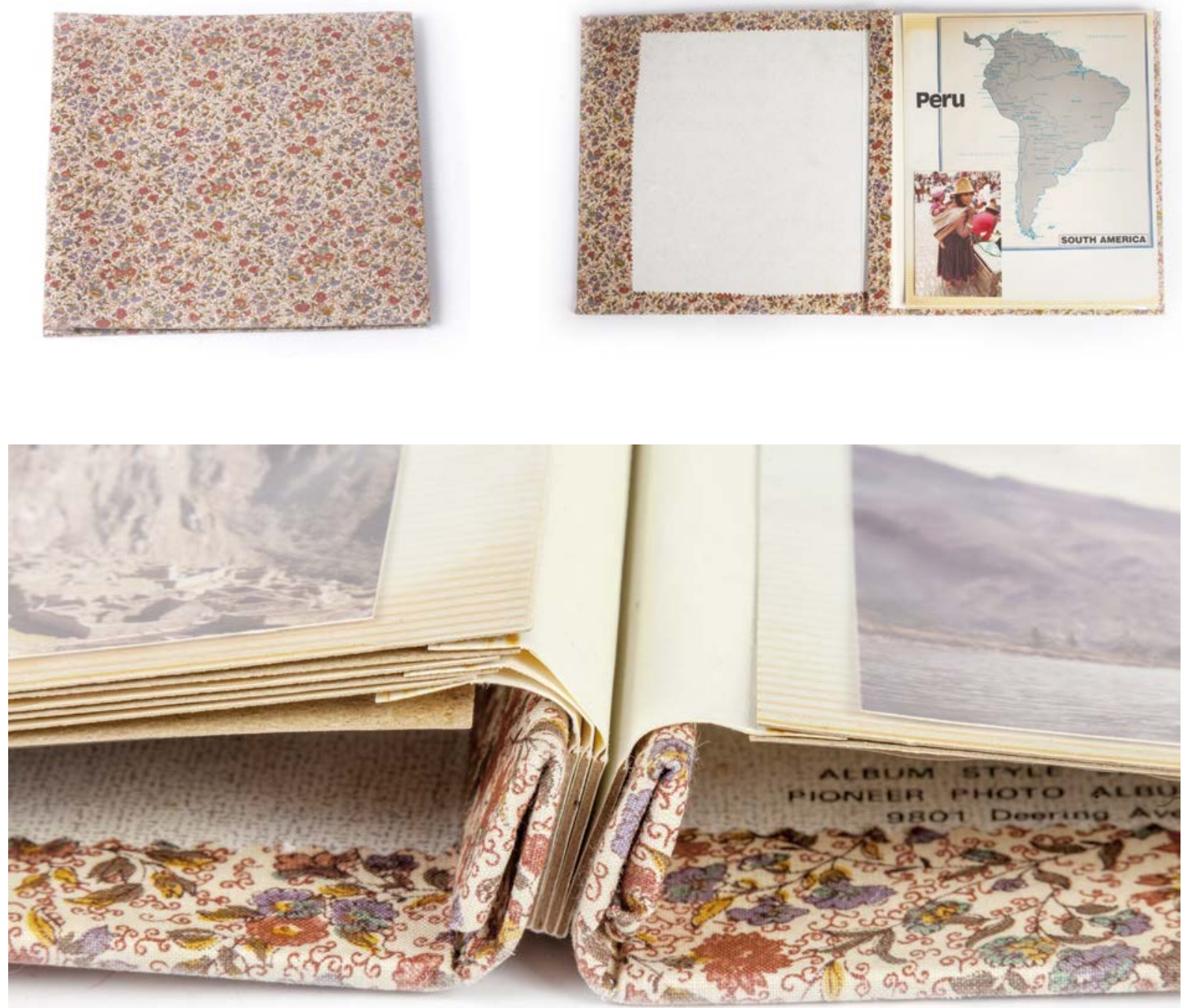

\section{COMPOSITING IN PHOTOSHOP}

If your document is too big to scan on your flatbed scanner, you can scan it in quadrants, allowing them to overlap approximately $30 \%$ or so. You may need more than four sections if the document is very large. ${ }^{37}$

37. Puglia, "Technical Guidelines for Digitizing Archival Materials for Electronic Access," 29. 

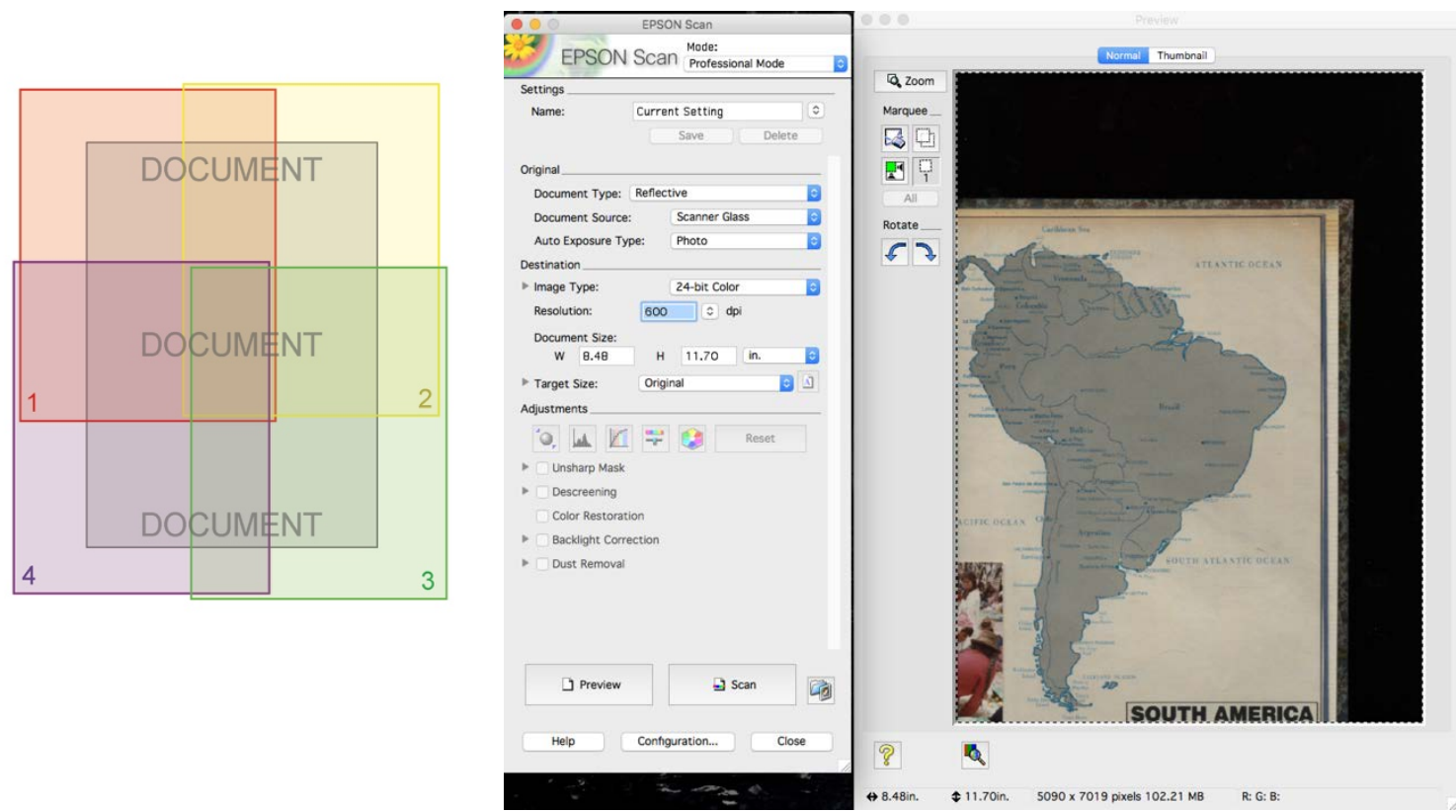

1. Scan the document in four sections, as shown above. Each scan should be a tiff with the appropriate resolution and colour bit depth. To learn more about resolution and bit depth, please refer to the appendices.

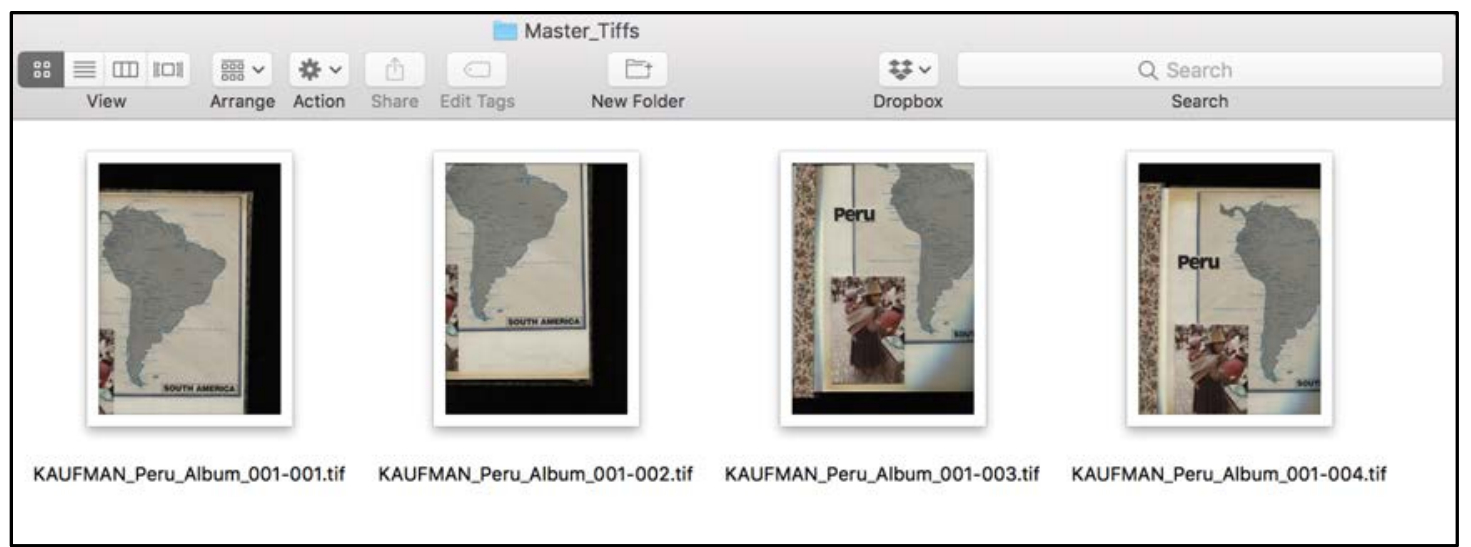

The four scanned sections.

2. Once you have scanned all four sections, open them in Photoshop.

\begin{tabular}{|l|lr|}
\hline Photoshop CC & File Edit Image Layer & Type Select \\
\hline & New... & $\mathscr{H} \mathrm{N}$ \\
\hline PS & $\begin{array}{l}\text { Open... } \\
\text { Browse in Bridge... } \\
\text { Open as Smart Object... } \\
\text { Open Recent }\end{array}$ \\
& Close & \\
\hline
\end{tabular}

a. Open the Photoshop application.

b. Go to FILE and then click OPEN. 
3. In the dialog box that now appears, location the four scans you have just created. Select them and then click OPEN.

\begin{tabular}{|c|c|c|c|c|}
\hline 国 嵒 $\equiv$ & Maste & Tiffs & & Q Search \\
\hline Name & 人 & Date Modified & Size & Kind \\
\hline [] KAUFMAN_Peru_Album_001-001.tif & & Apr 3, 2018 at $9: 30$ PM & 107.2 MB & TIFF image \\
\hline KAUFMAN_Peru_Album_001-002.tif & & Apr 3,2018 at $9: 31$ PM & $107.2 \mathrm{MB}$ & TIFF image \\
\hline 2. KAUFMAN_Peru_Album_001-003.tif & & Apr 3,2018 at 9:32 PM & $107.2 \mathrm{MB}$ & TIFF image \\
\hline 图 KAUFMAN_Peru_Album_001-004.tif & & Apr 3, 2018 at 9:32 PM & $107.2 \mathrm{MB}$ & TIFF image \\
\hline Options & & & Cancel & Open \\
\hline
\end{tabular}

4. If the files open as TABS in Photoshop, just drag the tabs down to "unpin" each file. I like to be able to see the four files I will be working with.
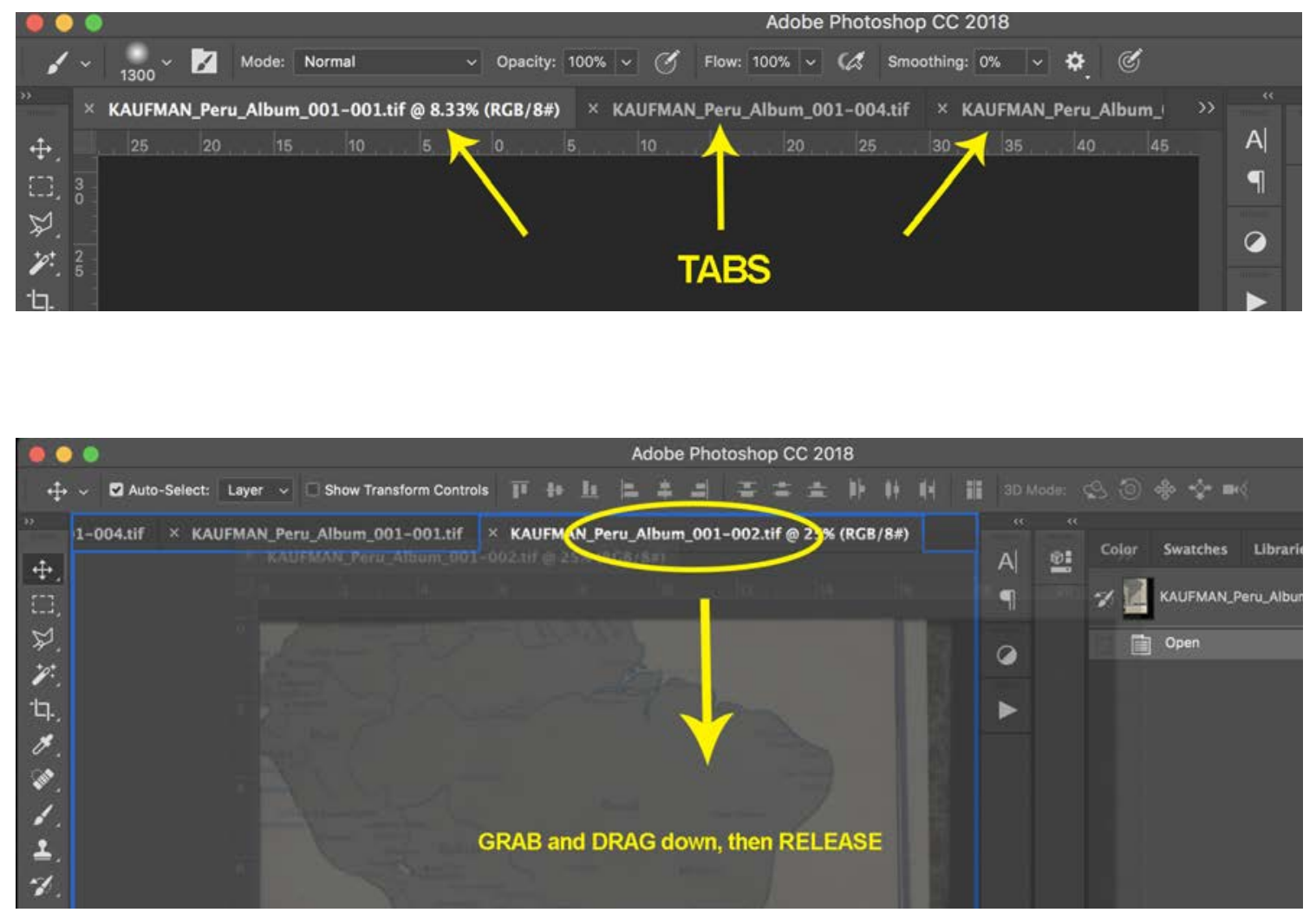

Drag the file tab downward to release it from the background interface. 


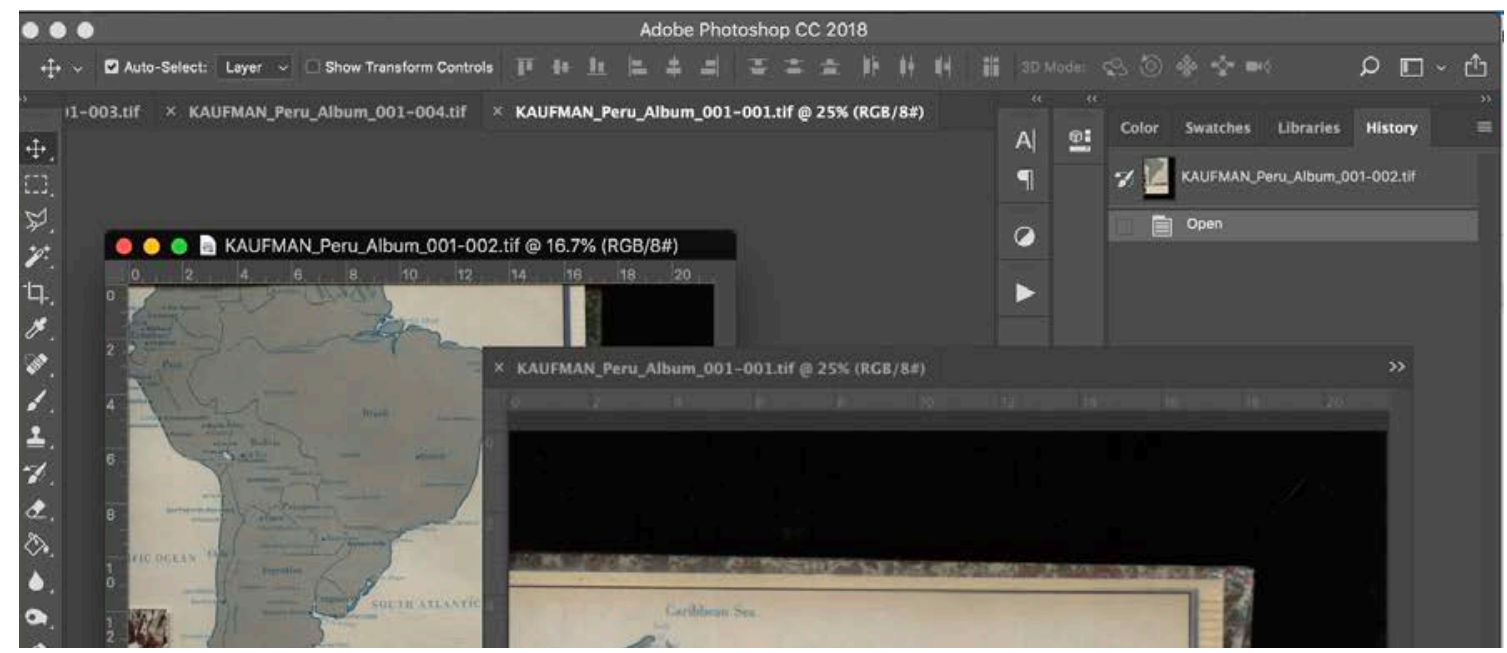

Files are now free-floating canvases on top of the Photoshop interface.

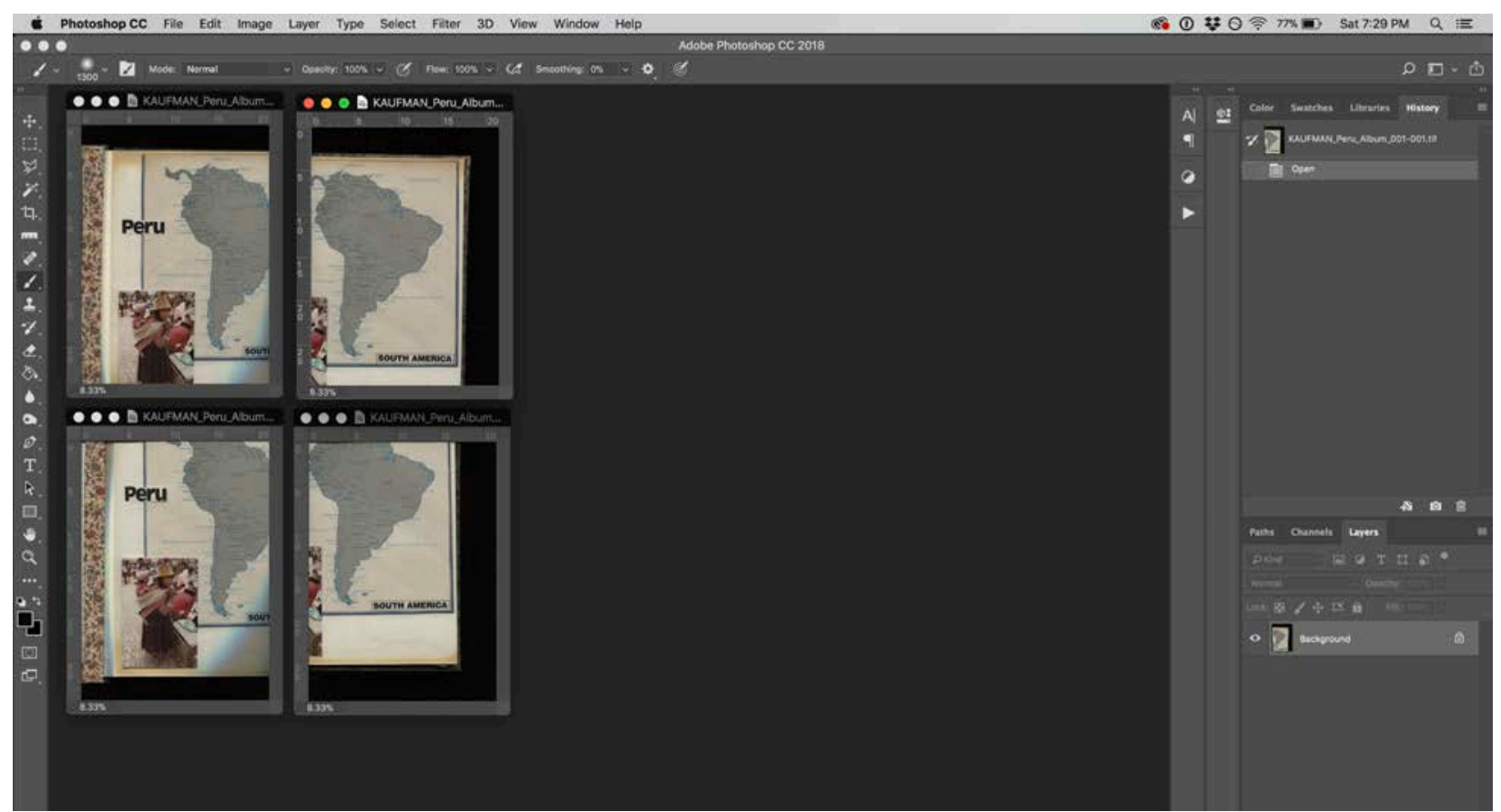

If you prefer to see all of the images you will compositing, drag the files down and use the magnifying glass tool to zoom out (or press COMMAND and - on a Mac, or CTRL and - on a PC).

5. Once the four component files are open, create a new canvas by clicking FILE and then NEW. 


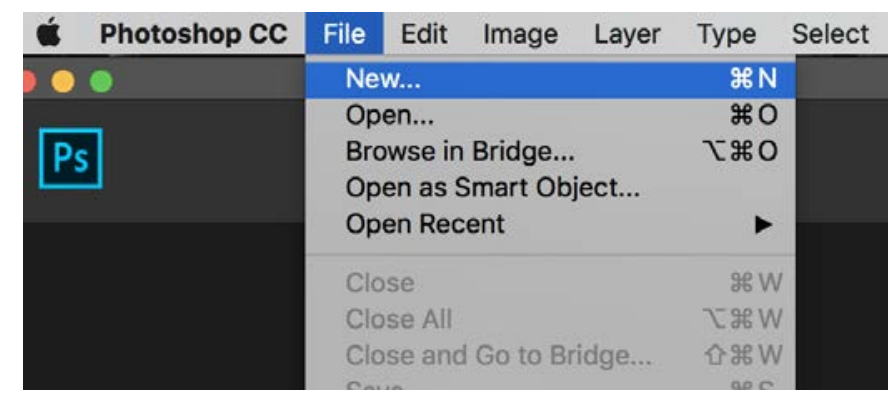

6. In the new document options menu that appears, name the document using your established naming convention and set the size of the canvas you want (you can always make this bigger or smaller later).

Make sure the resolution is the same as your scans. Because I scanned at $600 \mathrm{dpi}$, I have set the resolution to 600 pixels per inch. I have also selected RGB Color and 8 bit from the drop down menus because those are the settings at which I scanned the document. I have set the background colour to be black because I like all of my archival digitization files and scans to have a black border, but you can choose any colour you like. You can also change the background colour later. Once you have set the parameters of the new document, click CREATE.

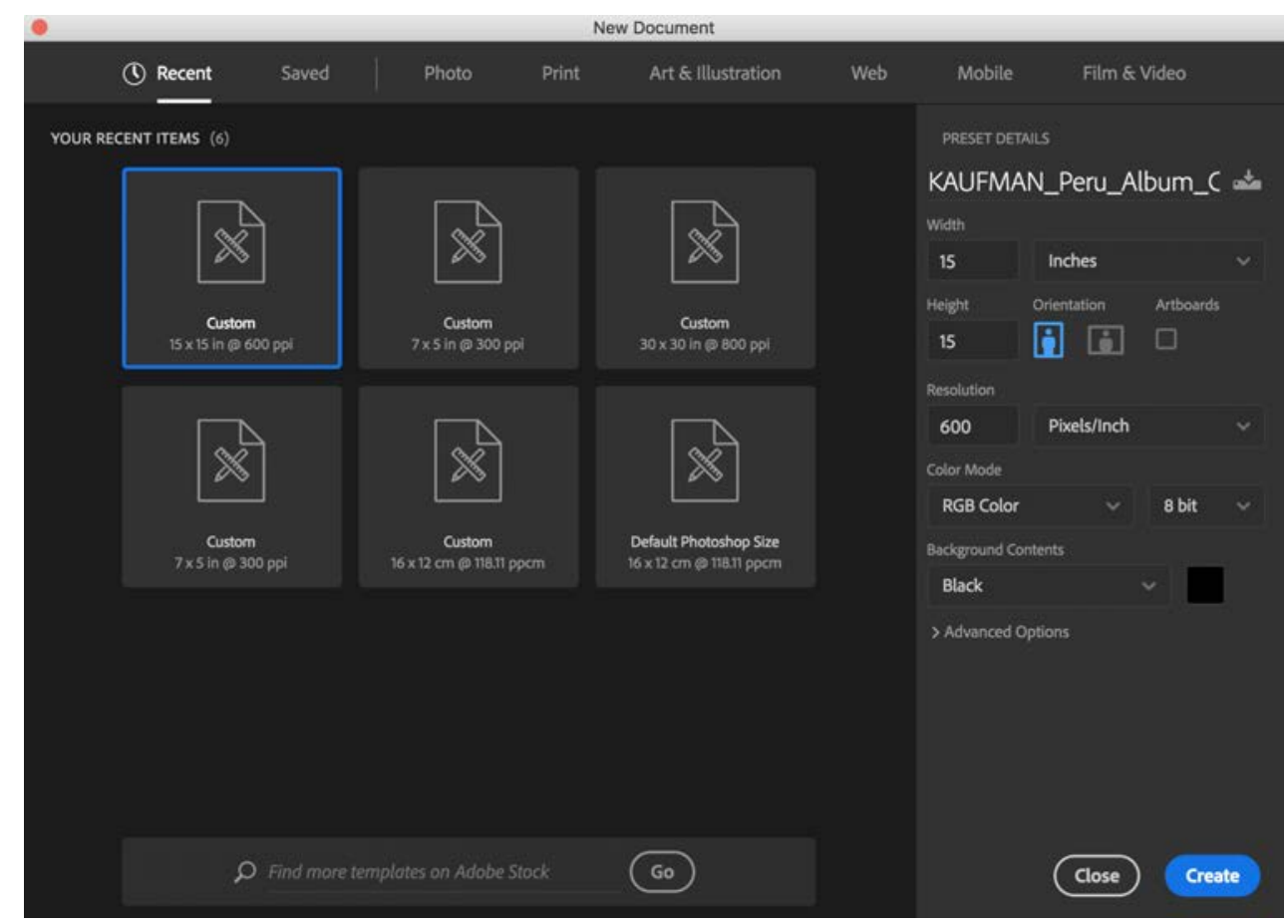

Create a new blank document. 


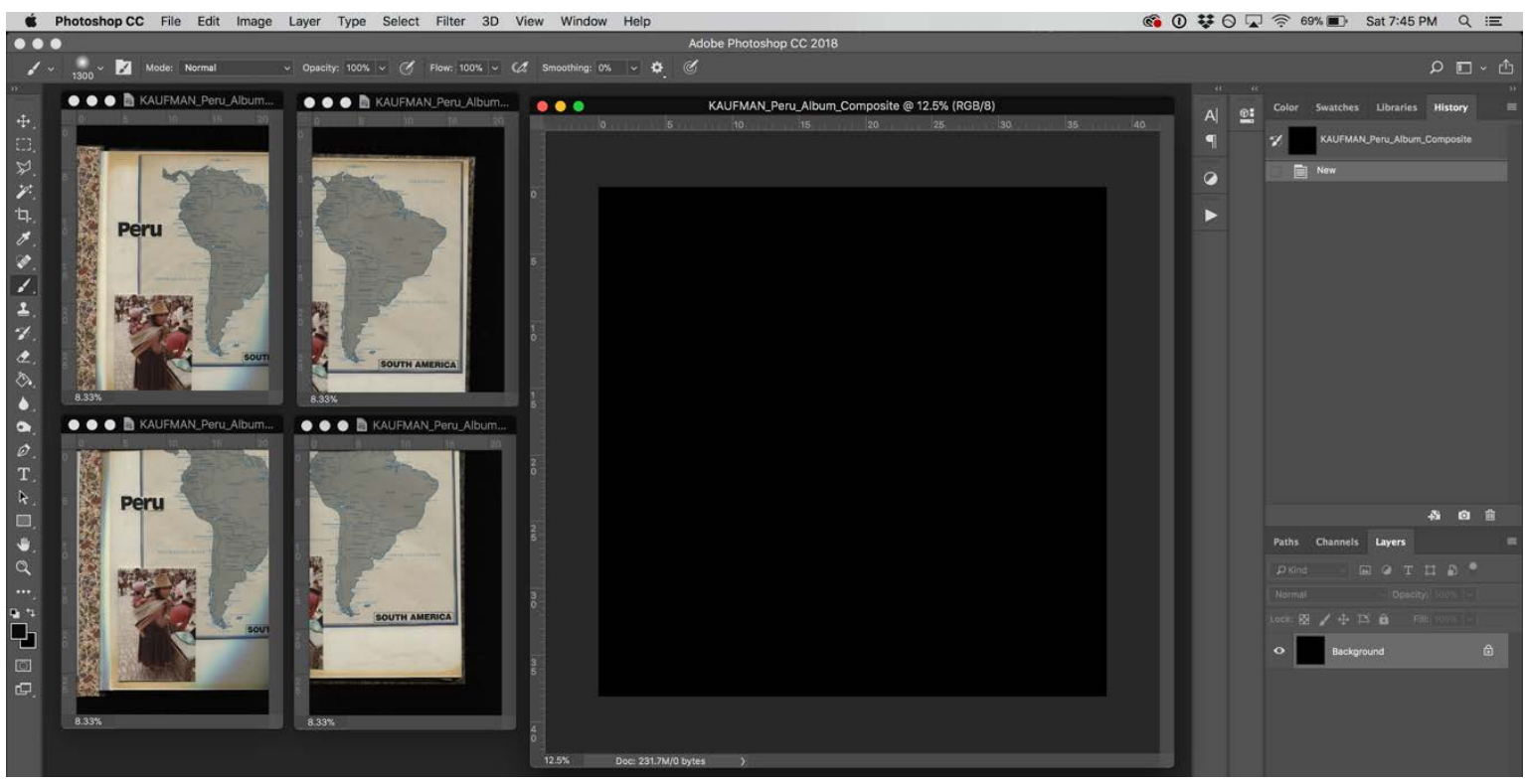

Blank document with the four files that will be composited.

7. To place the four images on the new canvas, select the MOVE tool from the tool menu, usually located on the upper left side of the application interface

8. Then click and drag each image onto the blank canvas. You will notice that each image is placed

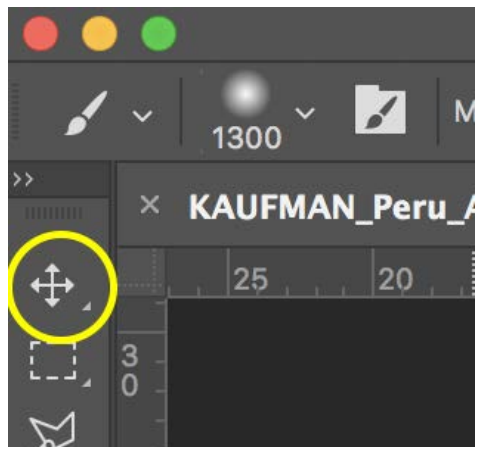
onto its own layer.

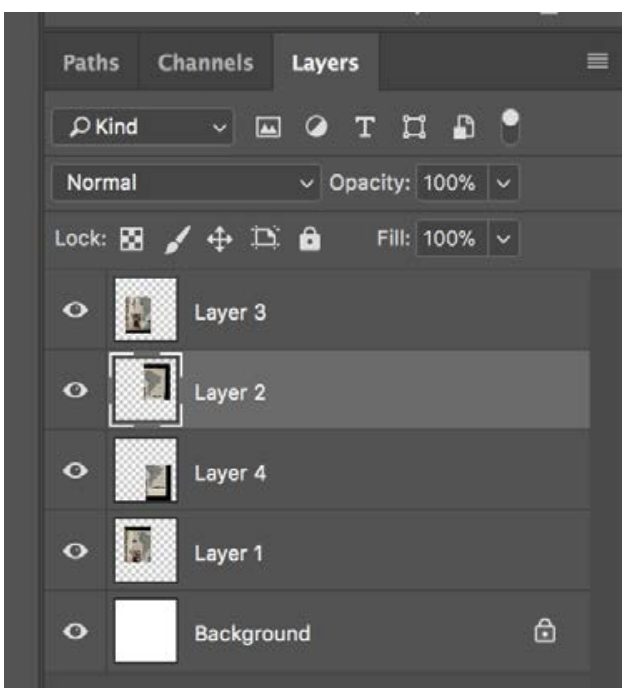




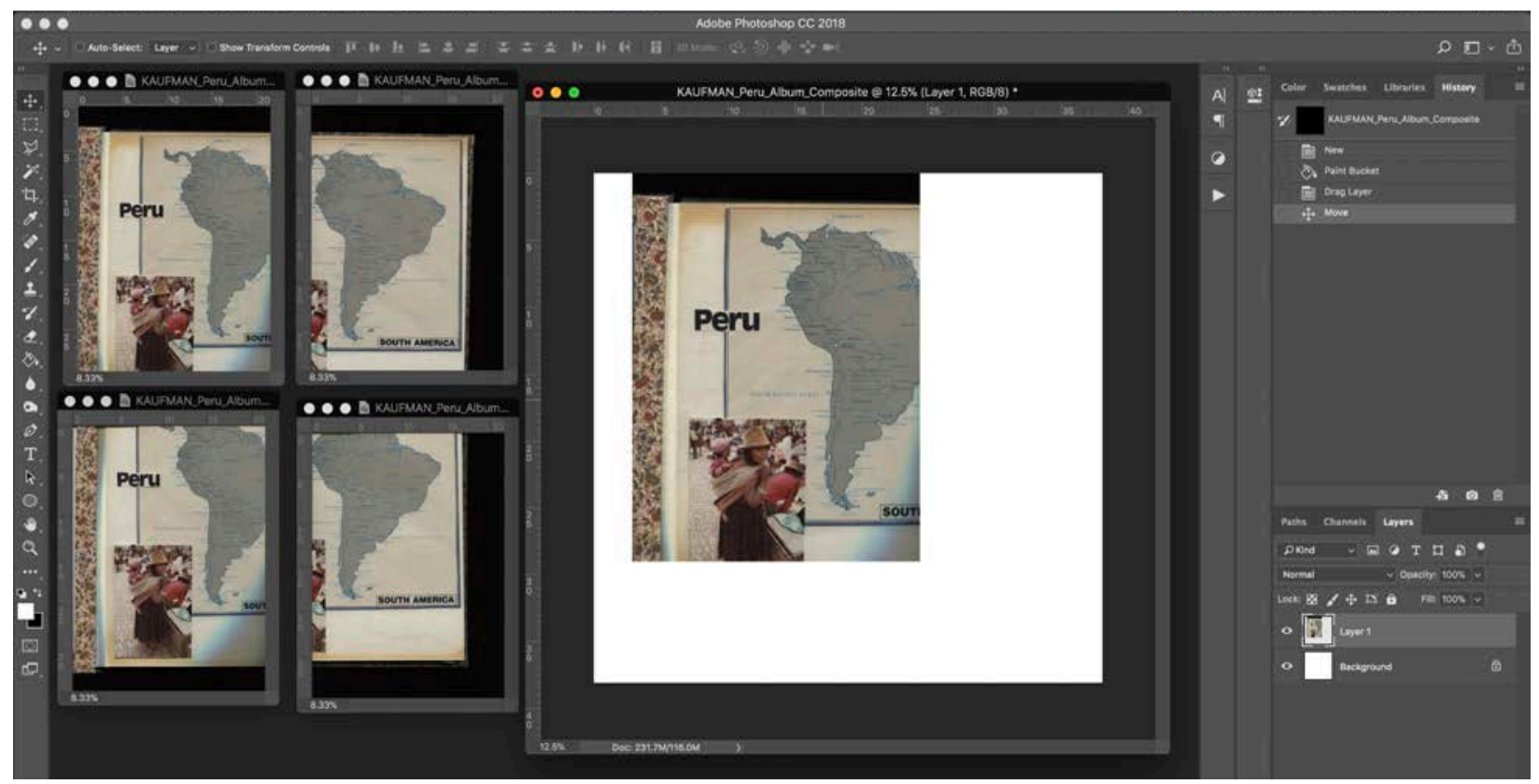

The first image has been dragged onto the blank document.

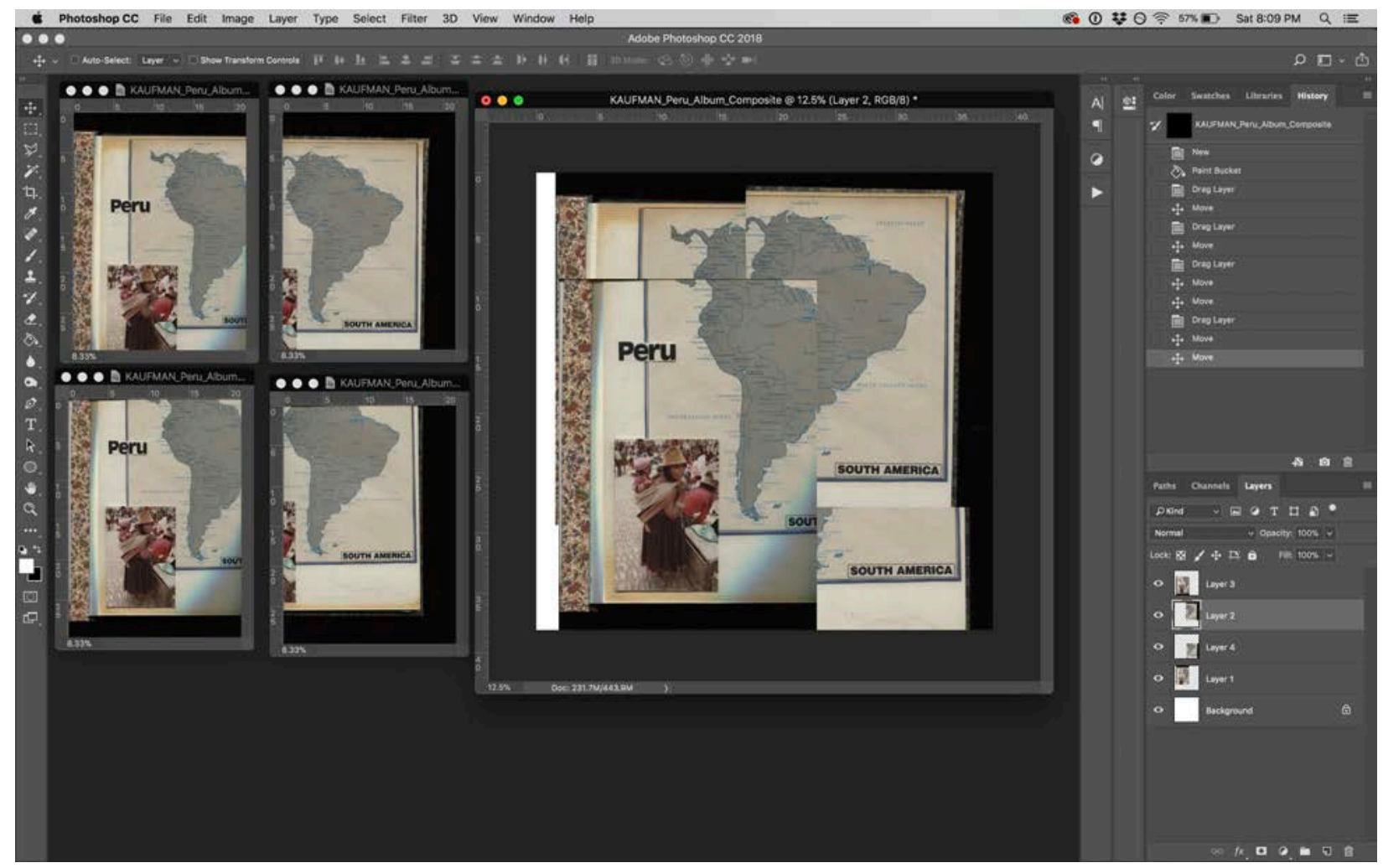

All four files have now been dragged onto the blank document (pictured here with a white background). 
9. If you want to make the canvas bigger so you can see the entirety of all four images, click IMAGE, then CANVAS SIZE.

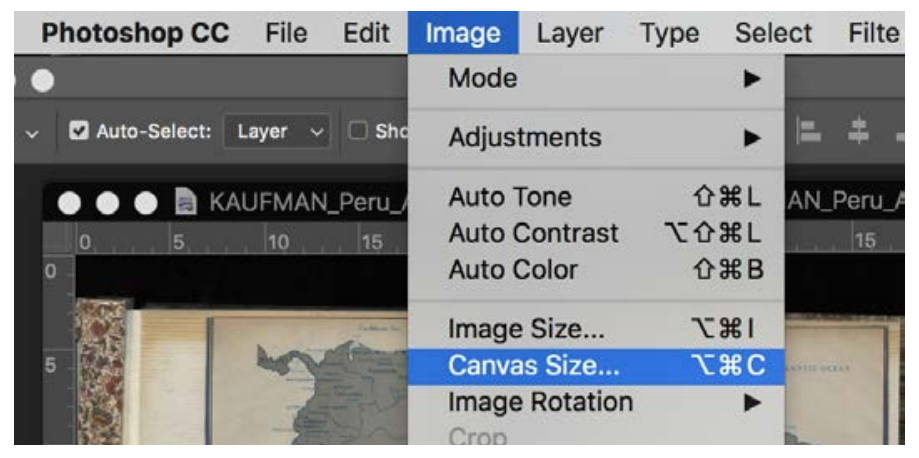

10. Type in the new size you would like the canvas to be. In this case I have increased the size from $15 \times 15$ inches to $20 \times 20$ inches. Then click OK.

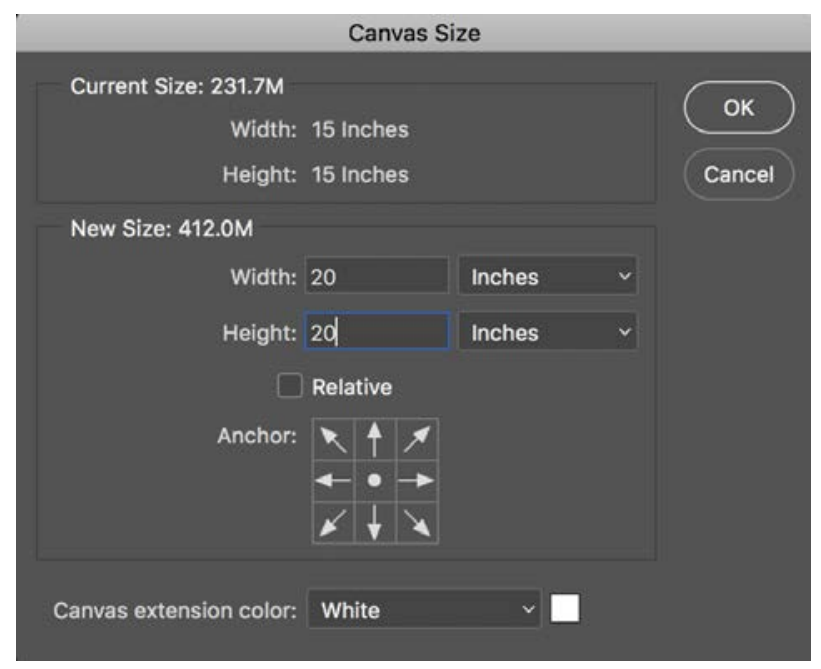




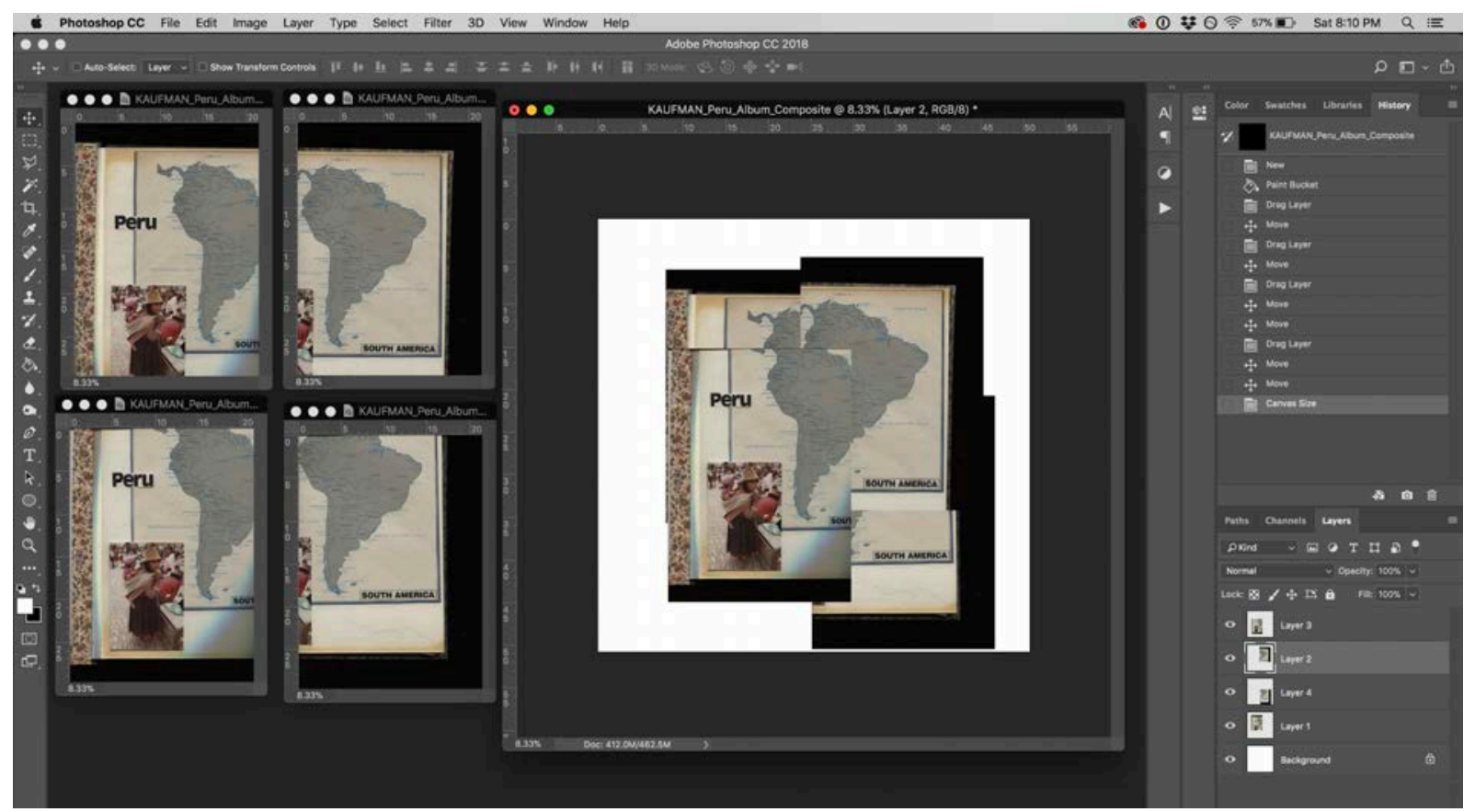

Now that the canvas size has been increased, all four files are completely visible.

11. To move each section, select the corresponding layer and then move (with the MOVE tool selected in the TOOLS menu). To move one image on top of another, select the layer it is on from the layer list and DRAG it up or down to change the layer order.

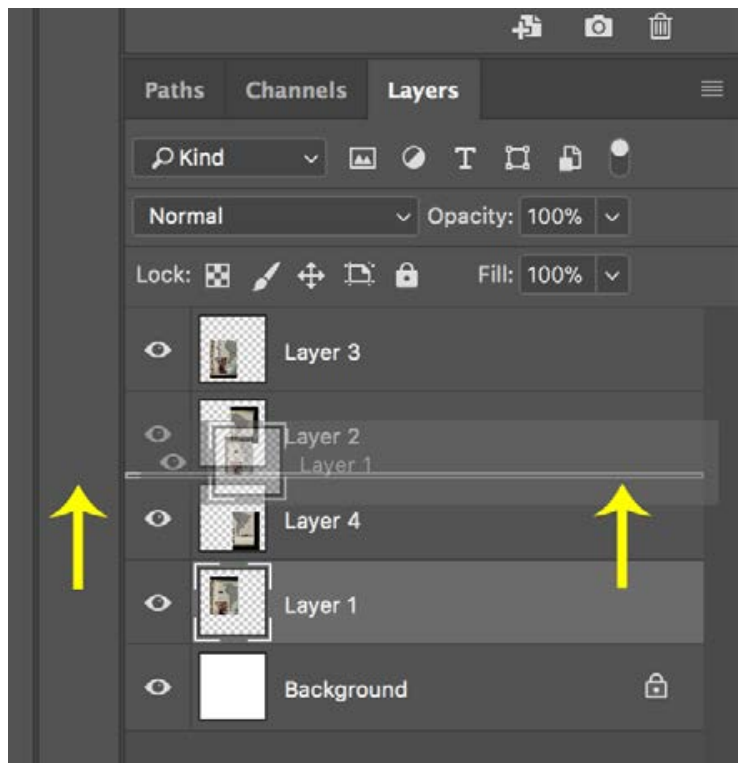


12. You may need to spend some time adjusting the order and position of each layer until they match up. Here I have added coloured outlines to illustrate the final layer positions and ordering that I found worked best for this page.
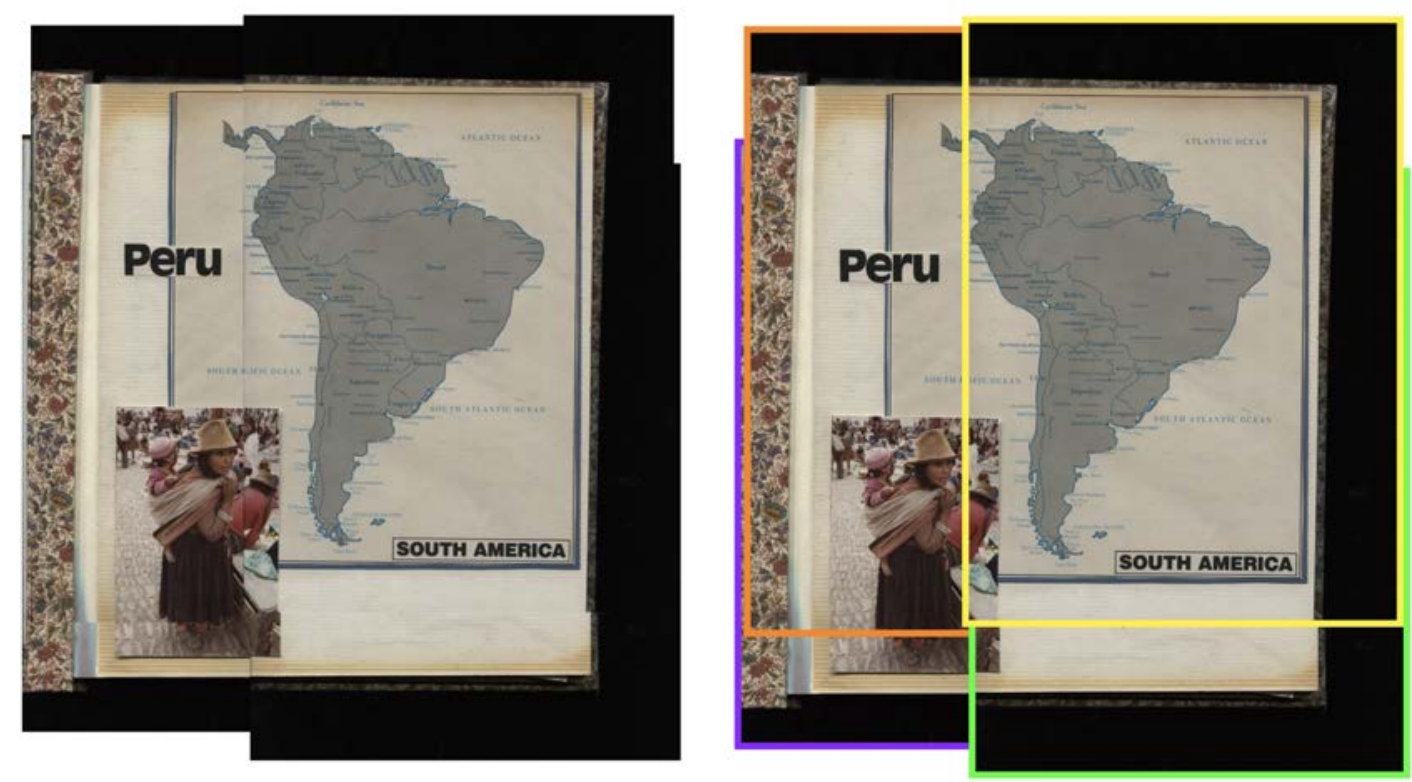

13. Just adjusting the order and position of the layers may not be enough to match everything up. If this is the case, you may need to use the TRANSFORM tool. This tool allows you to change the dimensions and positioning of a layer or object in different ways by clicking and dragging on anchors positioned around the image. The different transform types are scale, rotate, skew, distort, perspective, and warp.

14. When you are done creating your composite, save it as a PSD, TIFF, and JPEG. Saving it as a PSD will allow you to save the layers and adjustments in case you ever want to go back to the file and make adjustments. Saving the composite as a TIFF creates a PRODUCTION MASTER ${ }^{38}$. The master files themselves, following guidelines set by $F A D G I^{39}$, are the 4 images you composited together.

38. A 'Production Master' is an altered version of the 'Master' file. For example, if you scan a slide, that raw (unedited) scan is the 'Master'. Adjusting the colour, exposure, as so forth in Photoshop and saving a copy constitutes the creation of a 'Production Master'. FADGI (Federal Agencies Digitization Guidelines Initiative) recommends keeping both copies.

39. Thomas Rieger, "Technical Guidelines for Digitizing Cultural Heritage Materials: Creation of Raster Image Files." U.S. National Archives and Records Administration, Federal Agencies Digitization Initiative 
15. Save it as a Photoshop Document (PSD) by clicking FILE then SAVE AS.

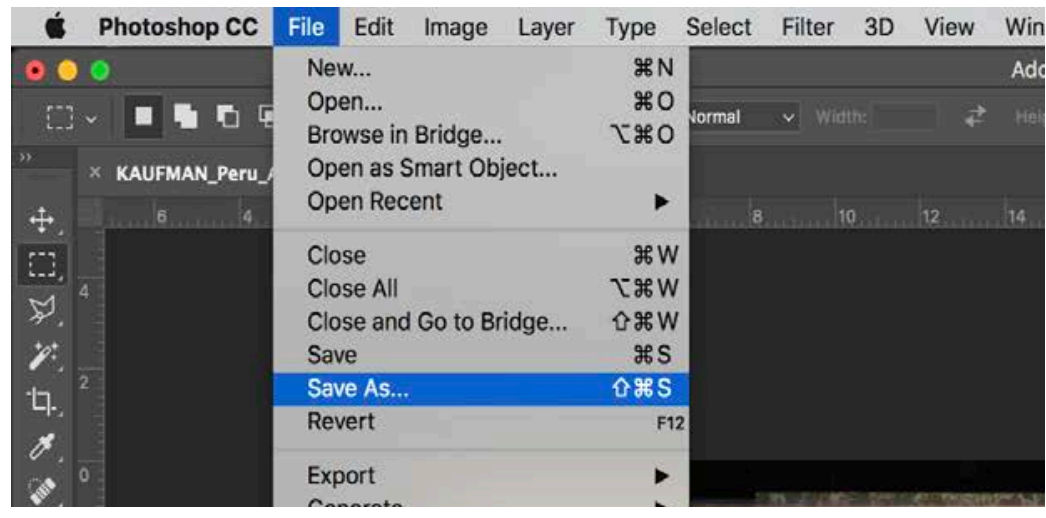

16. Name the document according to your established naming convention. I have called this document KAUFMAN_Peru_Album_pg01-comp. The composite of the second page will be called KAUFMAN_Peru_Album_pg02-comp, the third page _pg03-comp, etc.

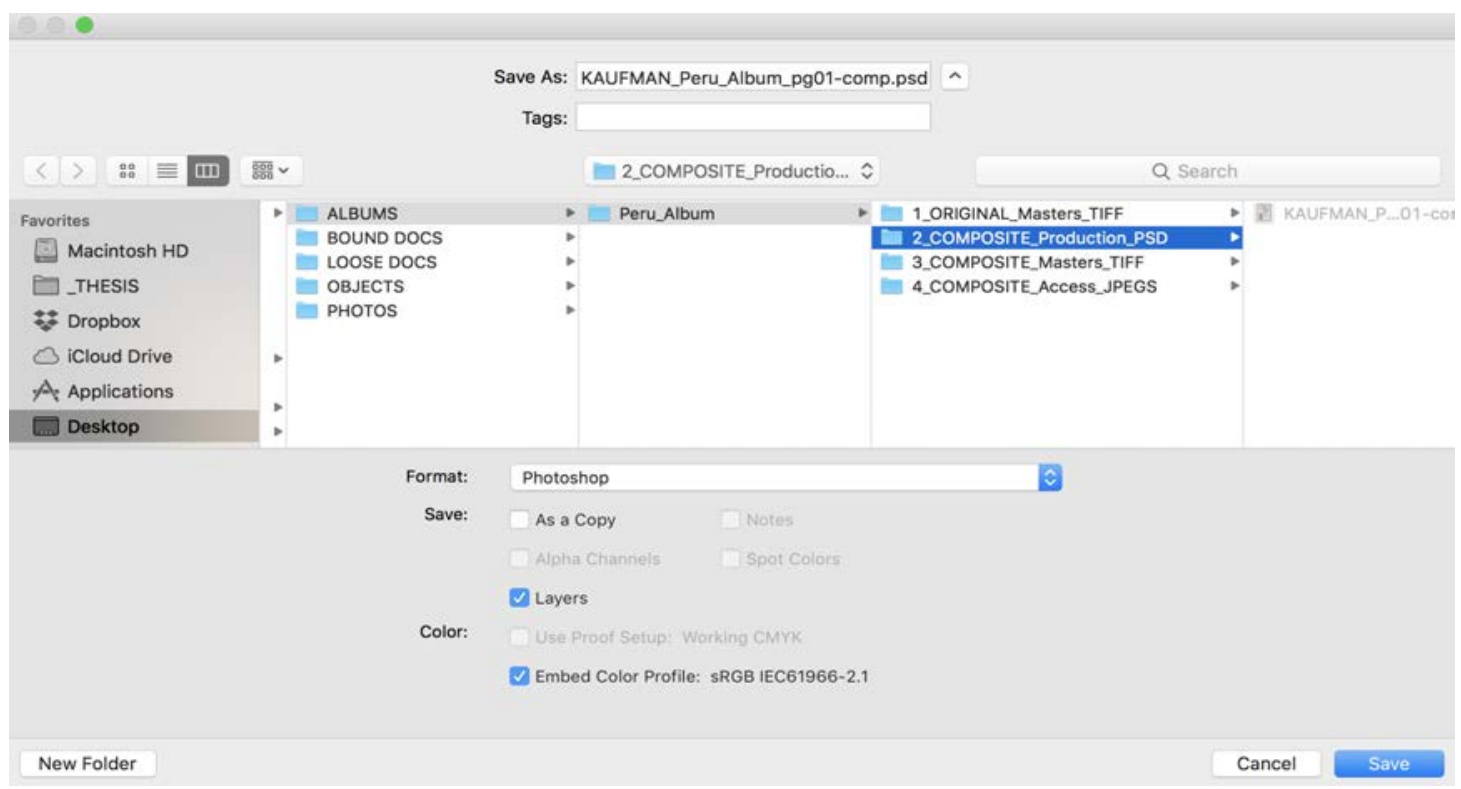

Notice that I have created a folders for each component of the composite process:

Still Image Working Group, http://www.digitizationguidelines.gov/guidelines/digitize-technical.html (Accessed June 16, 2018). 


\section{1_ORIGINAL_Masters_TIFF}

This is where I have saved the four original scans of the page. FADGI guidelines ${ }^{40}$ recommend that you keep these files in case you need to re-do the composite in the future.

\begin{tabular}{|c|c|c|c|}
\hline Peru_Album & $\begin{array}{l}\text { 1.ORIGINAL_Masters_IIFF } \\
\text { 2_COMPOSITE_Production_PSD } \\
\text { 3_COMPOSITE_Masters_TIFF } \\
\text { 4_COMPOSITE_Access_JPEGS }\end{array}$ & 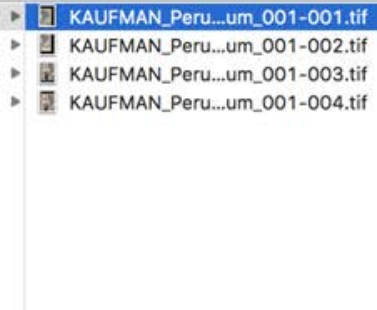 & KAUFMAN_Pe \\
\hline
\end{tabular}

\section{2_COMPOSITE_Production_PSD}

This is where I have saved the PSD file (which includes all of my layers and adjustments) that I have just created.

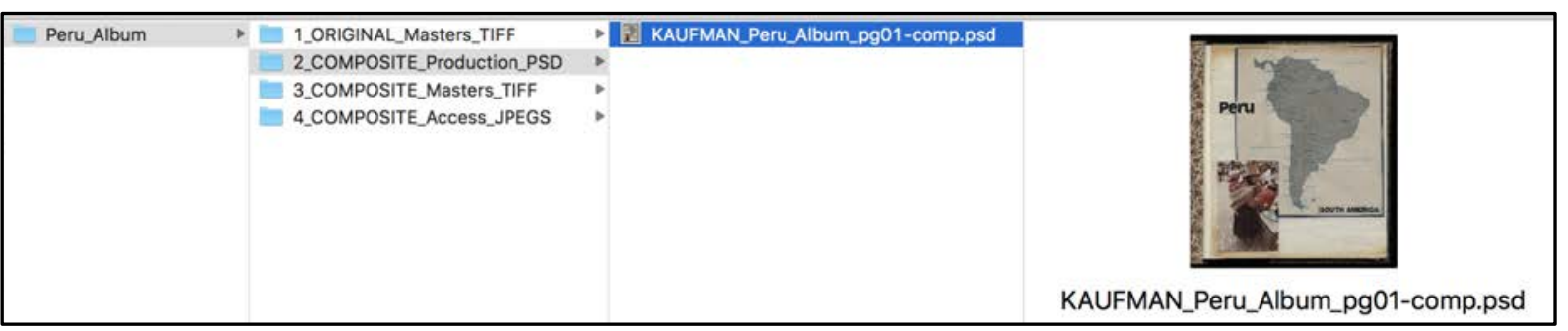

\section{3_COMPOSITE_Master_TIFF}

This where I have saved a TIFF version of the final composite. This version does not have layers. This file is not intended to be used, but rather kept as the new "archival master".

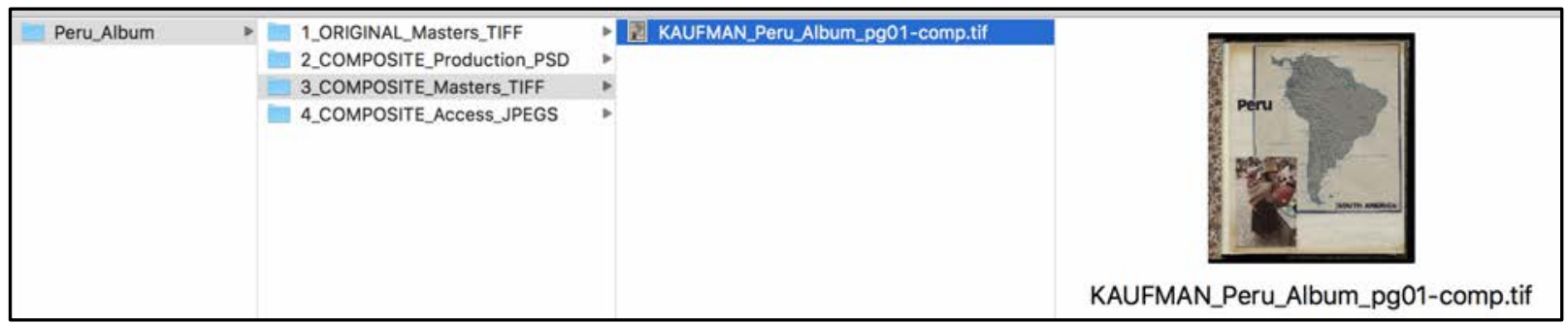

40 Rieger, "Technical Guidelines for Digitizing Cultural Heritage Materials," 29. 


\section{4_COMPOSITE_Access_JPEG}

This is where I have saved a JPEG version of each page. These are the files that I will use if I want to email, upload, share, or compile into a PDF.

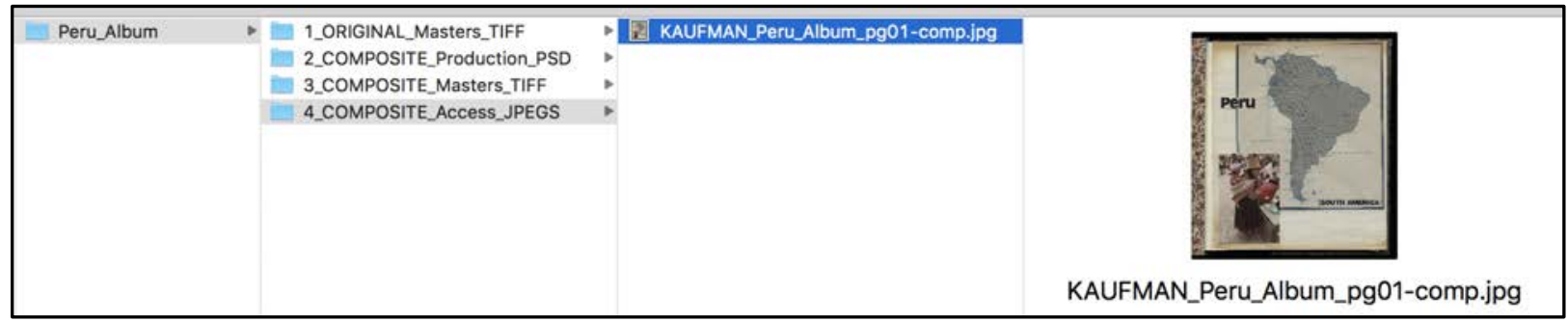

17. Now save the composite as a TIFF by clicking FILE then SAVE AS.

18. Select the folder where you want to save the document, then select TIFF from the Format drop-down menu and DESELECT the checkbox next to Layers. Saving the composite with layers is unnecessary since you have already saved the Photoshop version which includes layers. Saving the file as a TIFF without layers will also decrease the file size, and these files can get very big, very quickly. The file name can stay exactly the save. Because they are different file types with different file extensions (.psd, .tiff, and .jpg) the computer will know they are different files.

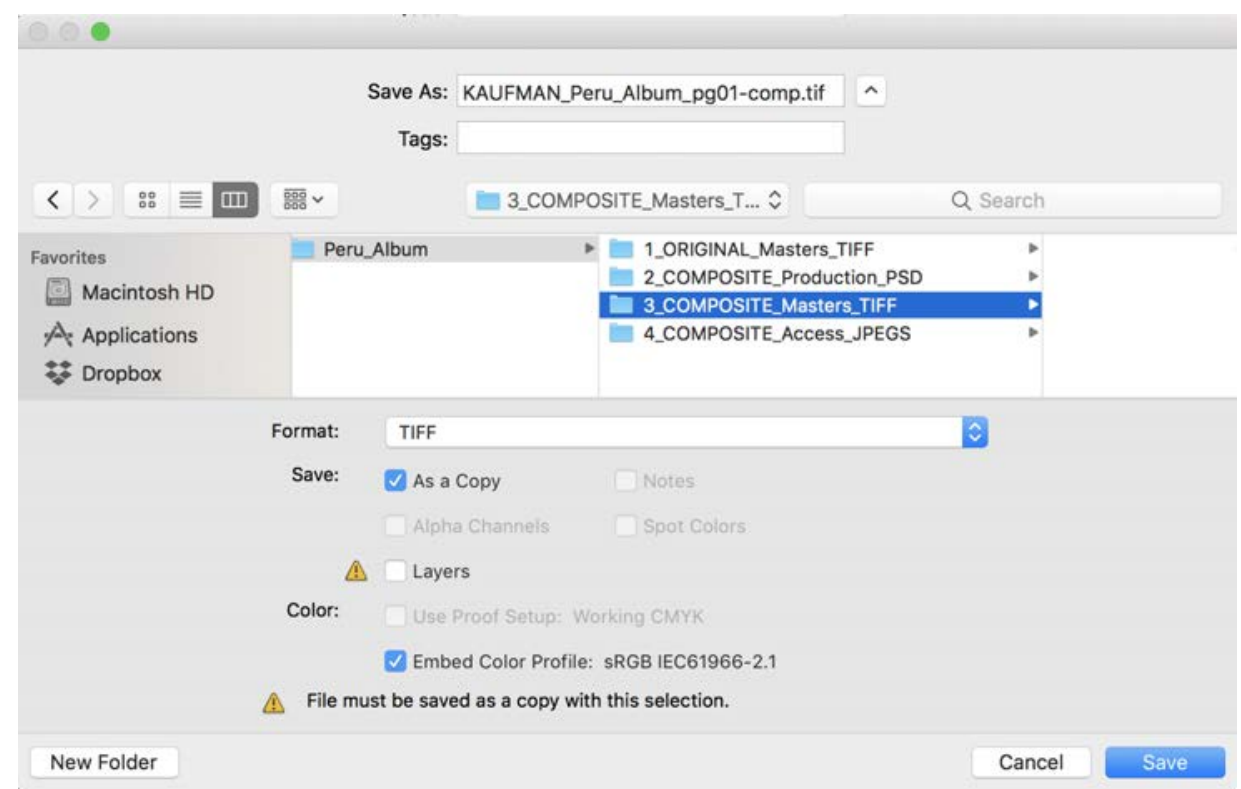


19. Click SAVE.

20. Next, the 'TIFF Options' menu box will appear. Select either NONE or LZW under Image Compression.

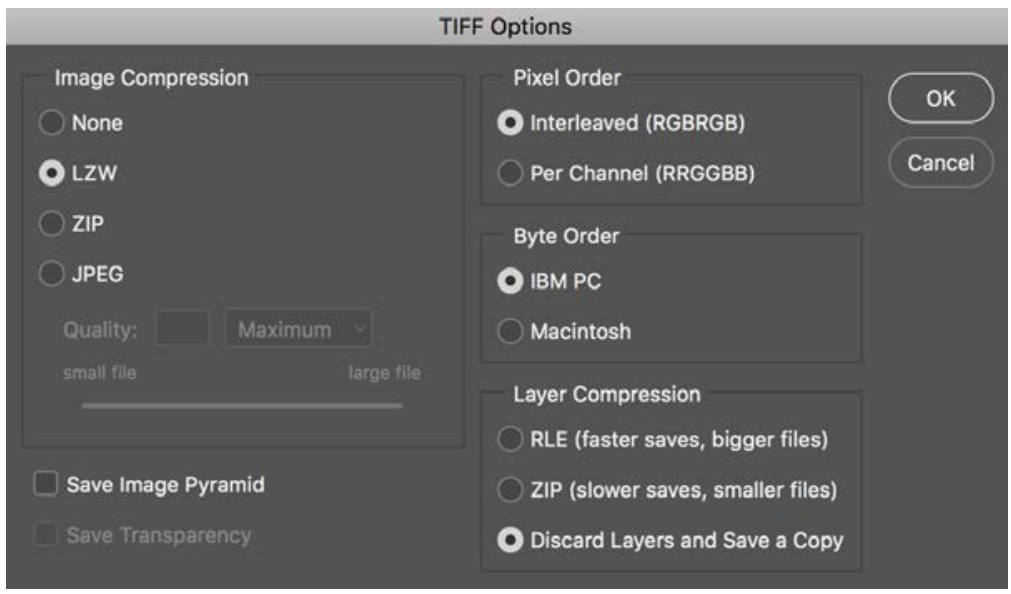

'No Image compression' is exactly what it sounds like. The image is not compressed at all. LZW compression is a type of lossless compression ${ }^{41}$ (it does not throw away any file data when compressed and saved) that decreases file sizes by roughly $30-50 \%$. In the past images compressed using the LZW algorithm may not have been compatible with different programs, but now most platforms support these files. If for some reason the LZW compressed TIFF gives you problems, you can always go back to the PSD file (which you have archived!) and save it as a TIFF with no compression.

21. Select Interleaved (RGBRGB) under Pixel Order.

Very occasionally some applications may have problems reading files saved with a Per Channel pixel order, so Interleaved is the most commonly used pixel order. For an lengthier explanation of the pros and cons of different pixel orders, please refer to the appendices.

22. Leave IBM PC selected as the default Byte Order.

41. "Lempel-Ziv-Welch (LZW) Compression," FileFormat.Info.

http://www.fileformat.info/mirror/egff/ch09_04.htm (Accessed May 1, 2018). 
When TIFF was a newly developed file format in the $1980 \mathrm{~s}^{42}$, it had to be saved differently depending on whether it was going to be accessed and used on a Mac or a PC computer. Now both types of computers can read both types of byte orders.

23. Select Discard Layers and Save a Copy under Layer Compression.

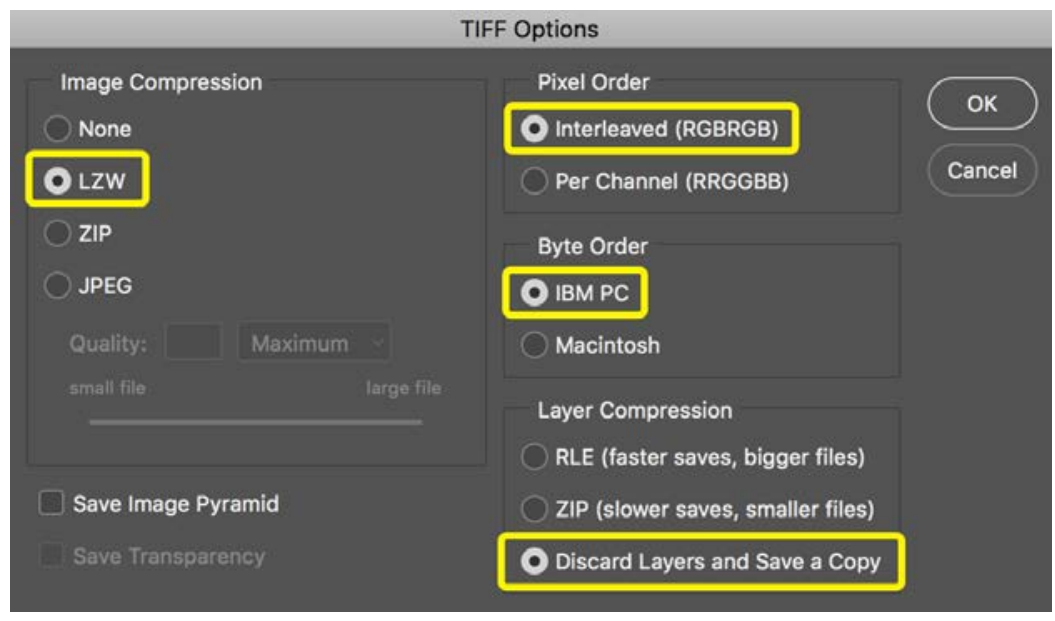

\section{Click OK.}

25. Finally, save a JPEG version of the composite by clicking FILE $\rightarrow$ SAVE AS

26. Select the folder where you want the file to be saved and then select JPEG from the Format

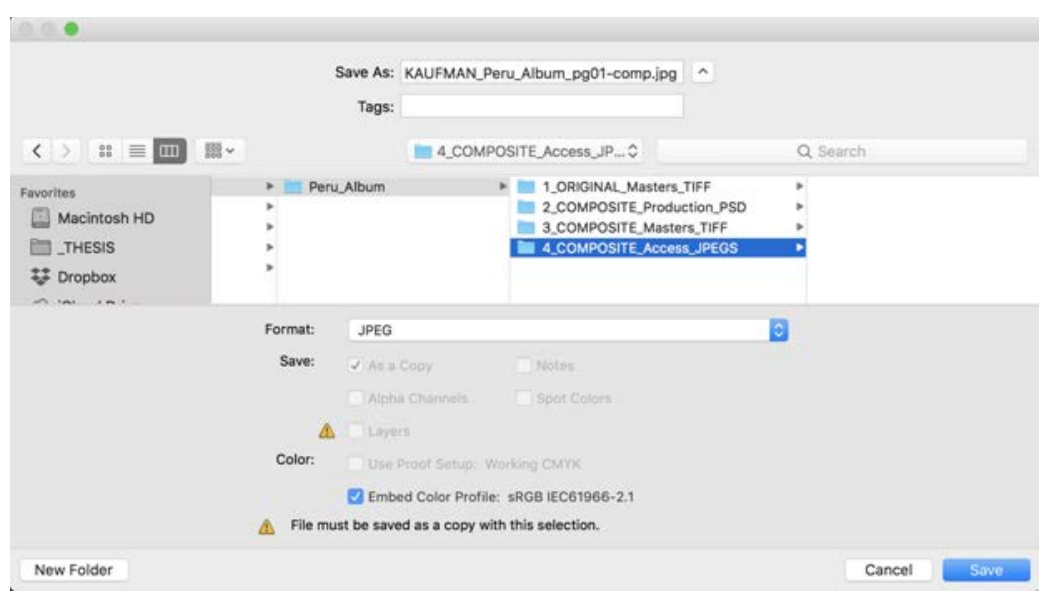
drop down menu.

\section{Click SAVE.}

42. "Sustainability of Digital Formats: Planning for Library of Congress Collections," Library of Congress, https://www.loc.gov/preservation/digital/formats/fdd/fdd000022.shtml (Accessed May 1, 2018). 

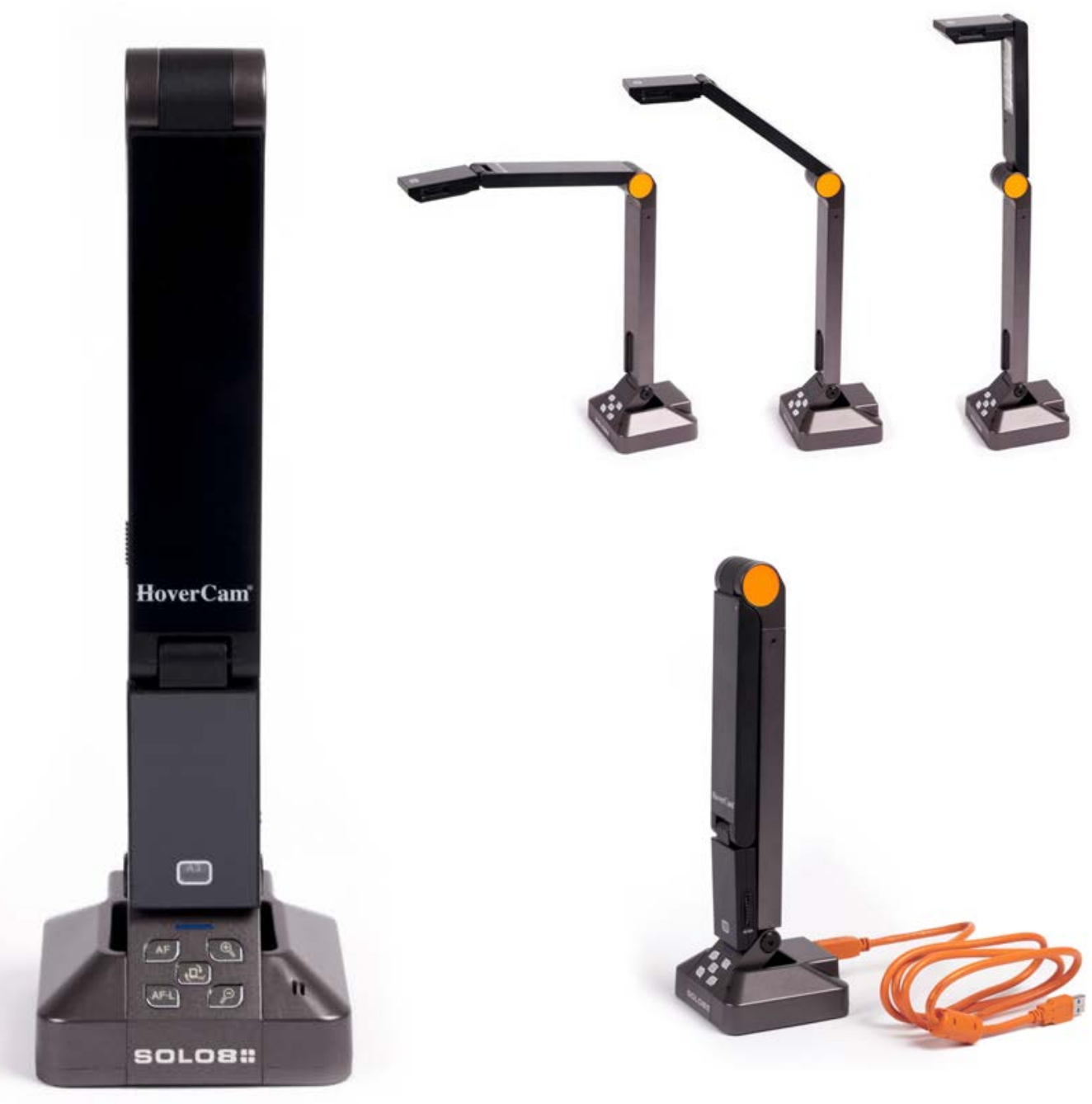

While there are many options available for digitizing documents and books, like the Fujitsu ScanSnap book scanner and the Epson Workforce portable document scanner, most of them only scan as PDF and Jpeg file formats, not as TIFFs. While PDF and Jpegs are both useful, they are lossy file formats, they are not archival. To learn more about archival and non-archival file types, please refer to Section 1.7: File Types. 
The HoverCam SOLO 8Plus is a document scanner that uses a small 13 megapixel camera to capture images and video. It has two adjustable components that allow you to scan a wide variety of different sized documents. It can also function as a webcam or video recorder. The installation software is built in to the base of the device, and it connects to both Mac and Windows via an included USB cable.

I tested the HoverCam for the Ontario Genealogical Society which was looking for an affordable, easy-to-use document scanner that captured archival lossless files (TIFF) and could be easily shipped between their numerous branches. I found the device to be quite powerful though small (approximately 49.5 centimeters tall and weighing $1 \mathrm{~kg}$ ) and recommended that it be purchased for the Society. 
The HoverCam Solo 8PIus

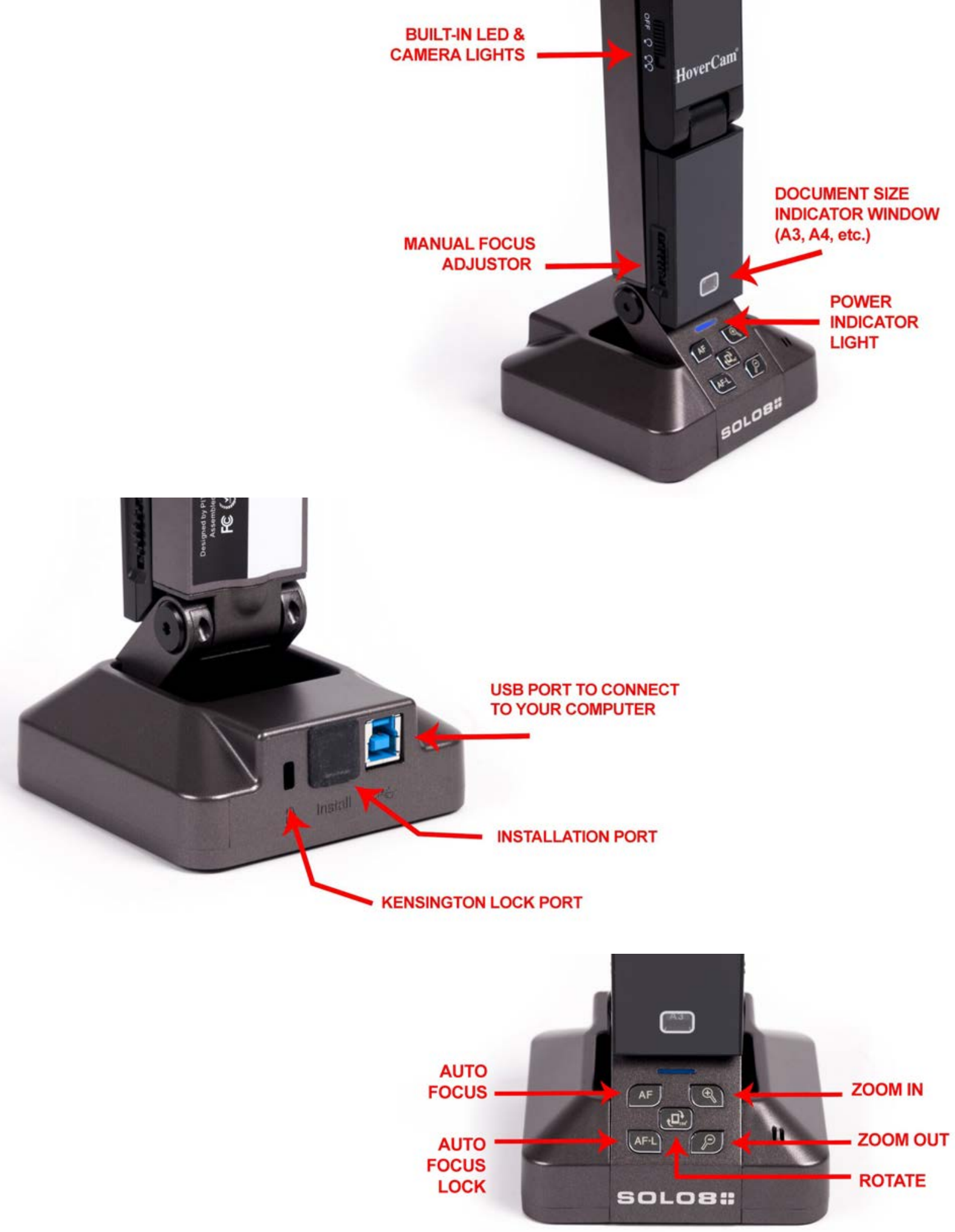


1. Open the HoverCam Flex10 program.

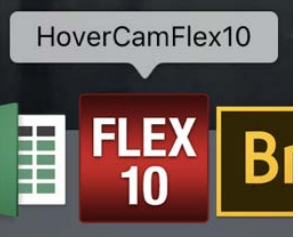

2. In the menu panel on the right, set the display size to either Maximum (16:9) or $4096 \times 3072$ depending on the size of the document you are scanning. If you unsure of which to use, you can easily switch between the two settings and see which better accommodates the document you are digitizing.

3. Disable Flicker Removal. ${ }^{43}$

4. In the Snapshot tab, select tiff from the drop down menu.
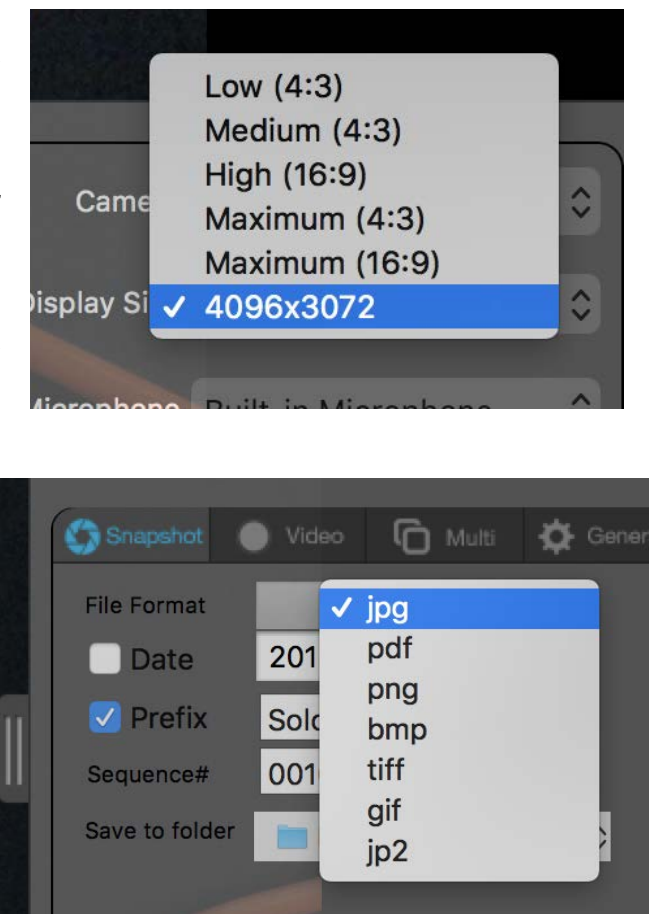

5. Input the prefix you want all the filenames to have. The prefix I used is "KAUFMAN_Diploma_" reflecting the collection and type of document I am scanning.

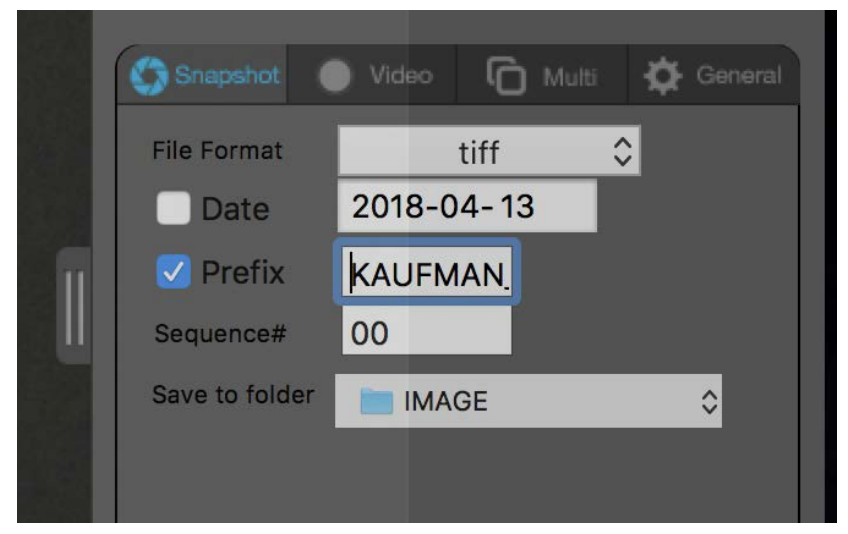

43. Flicker removal is most useful when capturing video because the setting is meant to compensate for subtle changes in lighting during a long exposure, time lapse or recording to capture an even exposure. Flicker removal is unnecessary when capturing still images because the exposure time is usually very short. 
Version $1.01 .020(08 / 25 / 2017)$ of the HoverCam Flex10 program does not let you save in any other location other than the HoverCam Flex10 folder that is created on your hard drive when you install the program, or any folder you create within that HoverCam folder. This is likely a bug in the programming and will likely be fixed in future software updates.

6. Place the document you want to scan under the camera. I covered the workspaces where I would be placing archival materials with a large piece of matte black cardstock. This protects the document from the table surface and allows me to capture images with a black border. It also prevents light from bouncing off of the table and affecting the images.

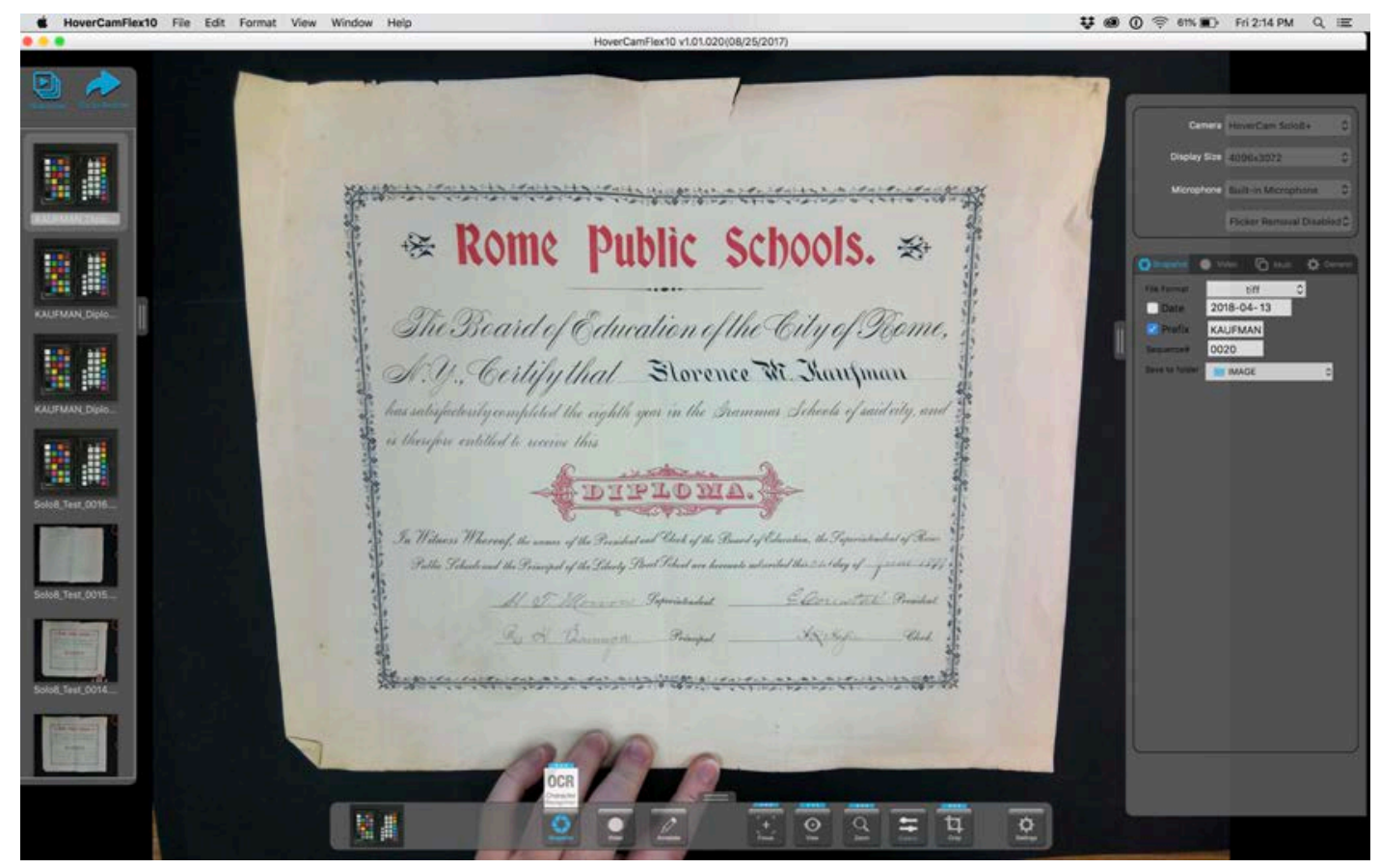

As you can see above, the document is distorted. This is because the $4096 \times 3072$ display size is essentially the widest angle the camera is capable of capturing, so areas farther away from the camera are subject to perspectival distortion. To fix this, I placed two binders underneath the black paper to angle the document surface back towards the camera. 


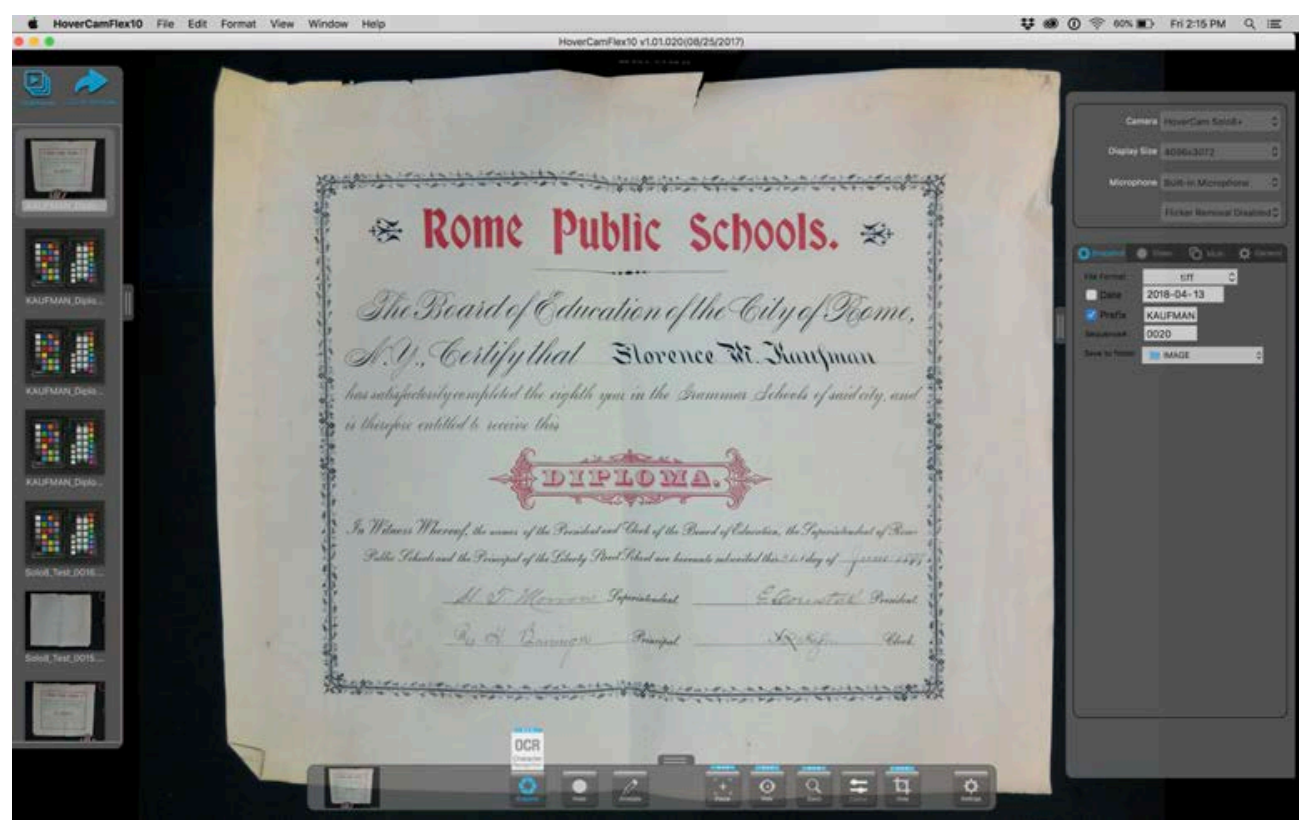

7. You can either focus the camera using the manual dial on the side of the device, but the built-in autofocus works very well. To check the focus or select a specific area of the document on which to focus the camera, click the Focus button at the bottom of the screen, and then either AF to prompt the camera to re-focus, or the focus symbol to manually select an area of the document.

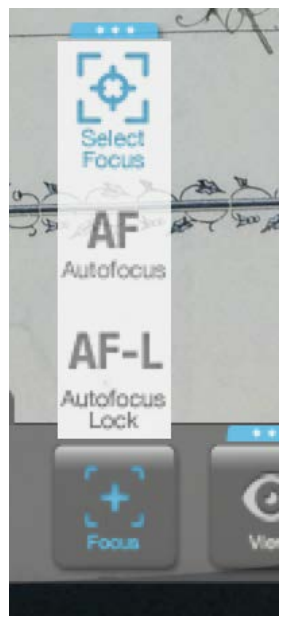

If you are doing a series of similar documents, you may consider using the autofocus-lock feature which prevents the camera from attempting to re-focus between each shot. This is also good to use if recording video to prevent the camera from adjusting in and out of focus when there is movement in front of the camera.

8. Once the document is in focus, click the Snapshot button. Thumbnails of the images will appear along the left side of the screen.

9. After you have taken the image, you can either continue to digitize other items, or continue on to post-processing (Section 3). 
Capturing TIFF files of the full capture area at the $4096 \times 3072$ resolution will produce very large files, about $210 \mathrm{MB}$. If your document does not fill the entire space, cropping the capture area around the document, leaving a small black border, will help reduce the file size and save space on your hard drive.

To CROP to a portion of the capture area, click the crop button, then click either the crop tool or the multi-crop tool, which allows you to select multiple areas to capture with a single click. Then use the cursor to click and drag a rectangle around the desired area. You can adjust the size and position of the crop window if needed.
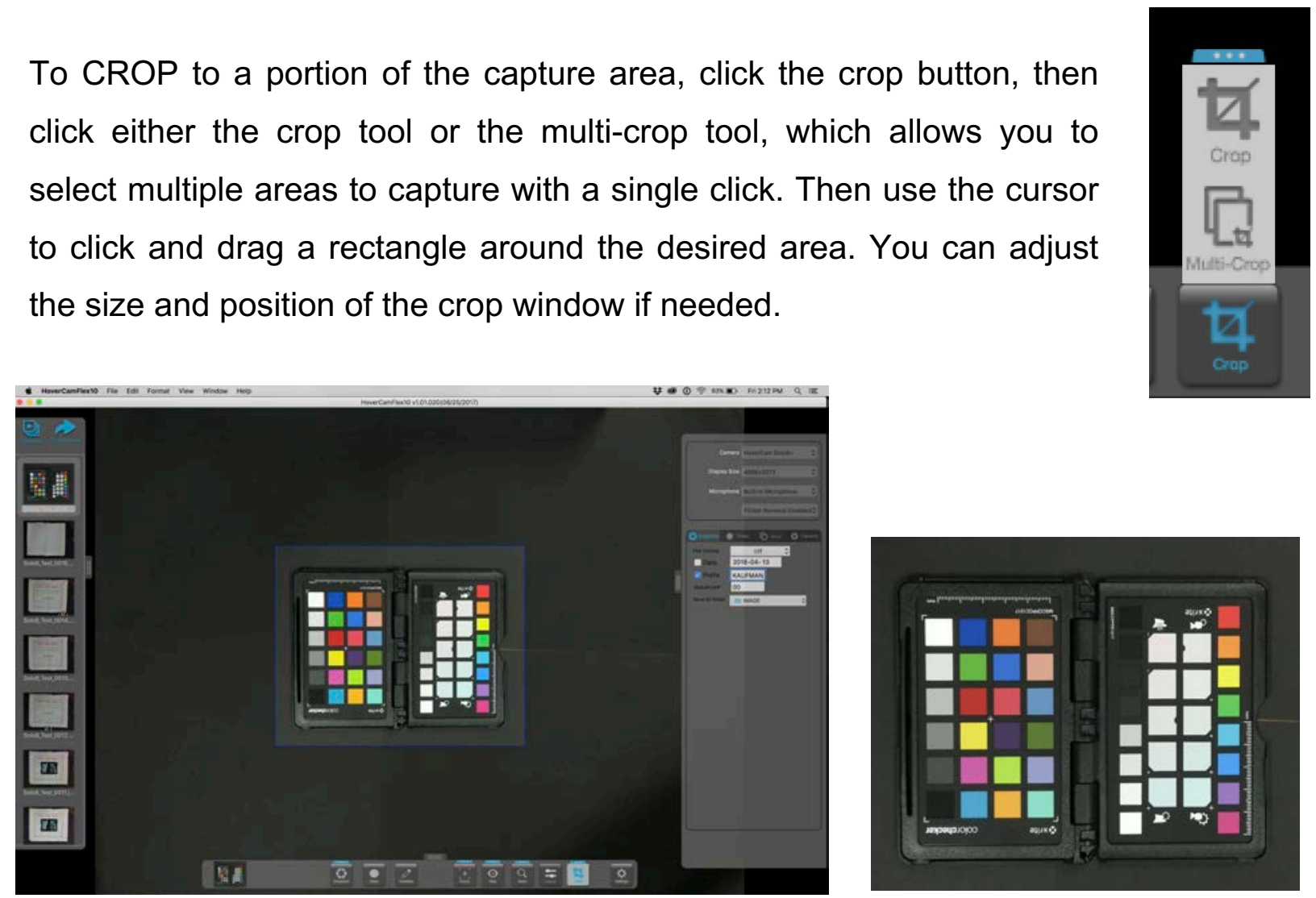

Capturing the entire visible space generates a 210 MB TIFF while the above cropped space is only $32 \mathrm{MB}$. 
To ROTATE the viewing area, click the View icon, then either the clockwise or counter clockwise buttons.

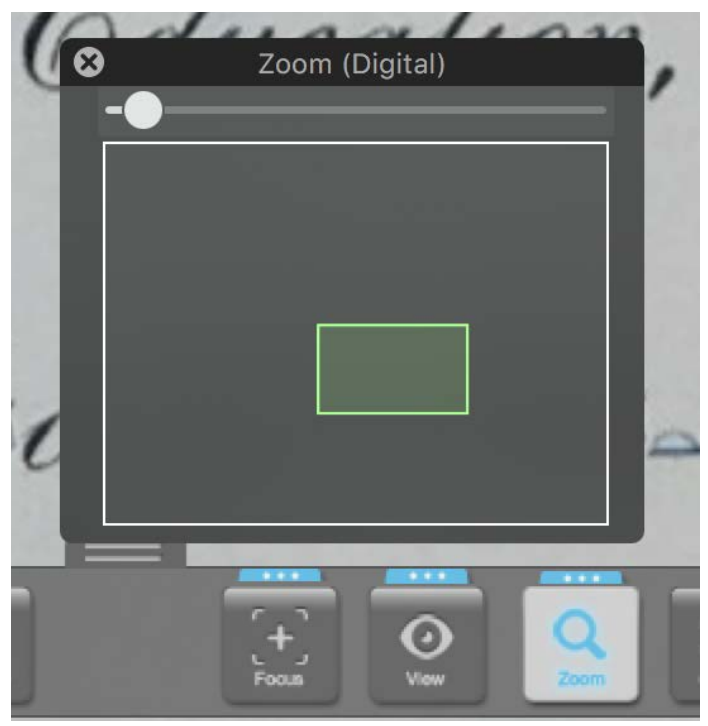

To ZOOM in or out, click the zoom button. A small viewing menu box representing the capture space will appear with a slider above to adjust the zoom distance. Note that even if the view is zoomed in, the whole capture space will be scanned (unless you have selected a specific area with the crop tool).
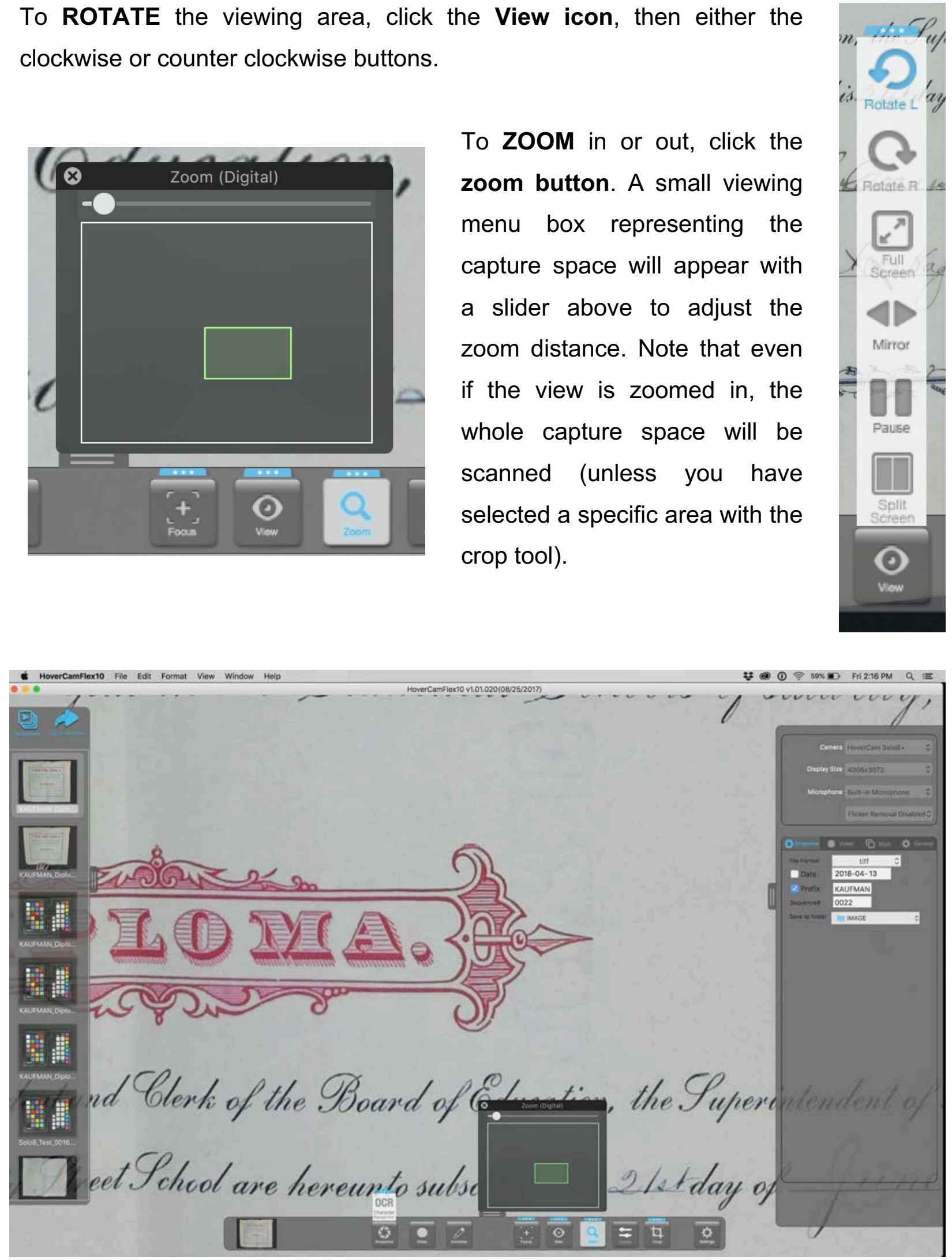


\section{ADVANCED CONTROLS}

In some cases you may want to make adjustments before capturing an image. I try to avoid this because it is difficult to be consistent when using manual adjustments, especially with programs like Flex 10, where the adjustment levels are only indicated by a slider, not numerical values. If any adjustments are needed, I prefer to use a more sophisticated imaging program like Photoshop or Lightroom. FADGI digitization guidelines also state that no adjustments should be made before capture so I use these functions sparingly.

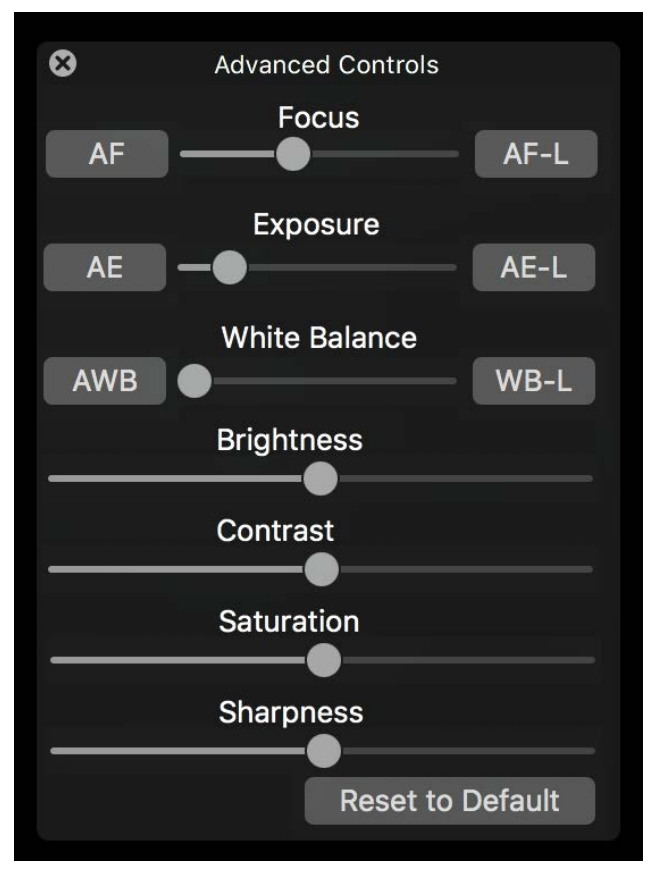

- Autofocus / Autofocus - Lock

- Auto Exposure / Auto Exposure - Lock

- Auto White Balance / White Balance - Lock

- Brightness

- Contrast

- Saturation

- Sharpness

\section{AUTOFOCUS (AF)}

Autofocus allows the camera to determine the most accurate focus for whatever is visible in the frame. Most of the time, the camera is able to focus more finely than human can, but occasionally you may experience glitches in the camera's autofocus in which case you may want to either focus manually or turn the camera off and back on, then let it try autofocusing again. 


\section{AUTOFOCUS LOCK (AF-L)}

Autofocus Lock allows the camera to determine the most accurate focus and then freezes that setting in place to prevent the camera from trying to re-focus every time an object in the frame is moved. This is useful if you are photographing a number of items that are all very similar-large, flat printed documents for example-since the camera's initial focusing will be accurate for all subsequent items.

\section{AUTO EXPOSURE (AE)}

Auto exposure allows the camera to determine the correct settings for an accurate exposure.

\section{AUTO EXPOSURE LOCK (AE-L)}

Auto exposure Lock allows the camera to determine the exposure settings and then freezes those settings to prevent them from changing. Again, this is useful if you are photographing many similarly-coloured or tones items under consistent lighting conditions to prevent the camera from re-evaluating the exposure each time and item is moved within the frame.

\section{AUTO WHITE BALANCE (AWB)}

Auto White Balance allows the camera to determine the most appropriate colour temperature and tint to produce an accurate white.

\section{WHITE BALANCE LOCK (WB-L)}

White Balance Lock freezes the current white balance settings to prevent the camera from re-measuring the white balance every time an item in the frame is moved.

\section{BRIGHTNESS}

Brightness adjusts the luminosity of an image, that is how much ambient light appears to be present. 


\section{CONTRAST}

Contrast is the degree of tonality change between the lights and darkest areas of an image. An image with deep black shadows and bright white highlights has more contrast than one with dark grey shadows and light grey highlights. Some old photographs and documents naturally have a low contrast due to the chemical processes with which they were made or due to age-related fading. When photographing these items it is best to record the contrast that appears in the item, whether that be high or low, rather than adjusting to what you feel looks most aesthetically pleasing.

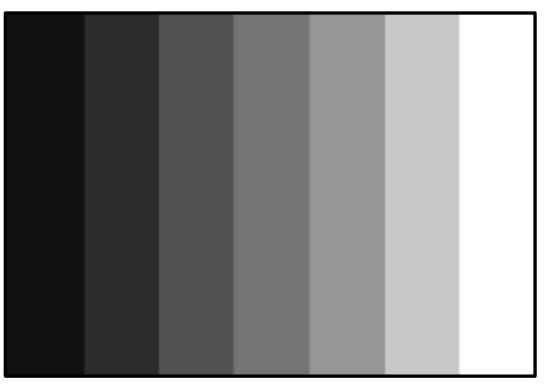

Normal contrast

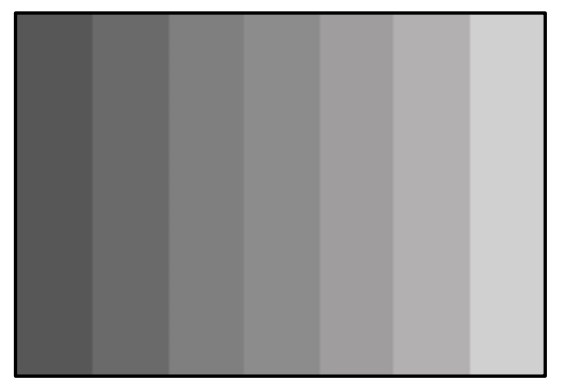

Low contrast, high bright

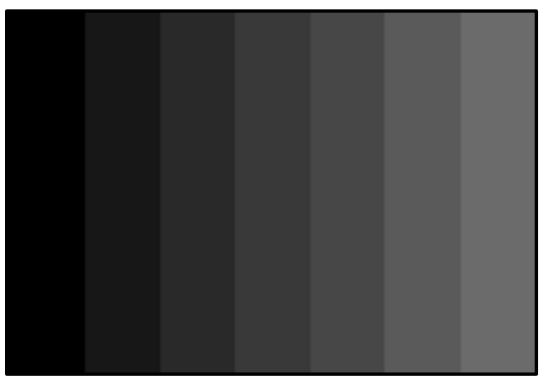

Low contrast, low brightness

\section{SATURATION}

Saturation refers to the depth or richness of a colour. Muted colours have a lower saturation than those that are rich and vibrant.

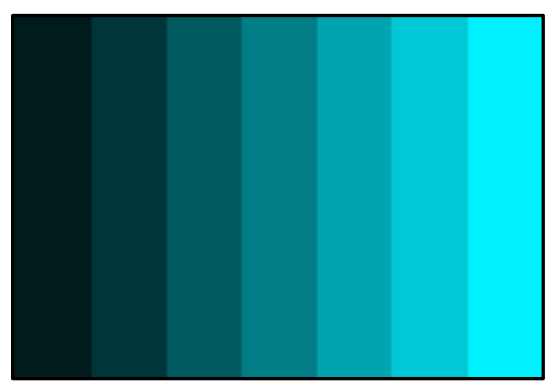

Normal contrast, high saturation

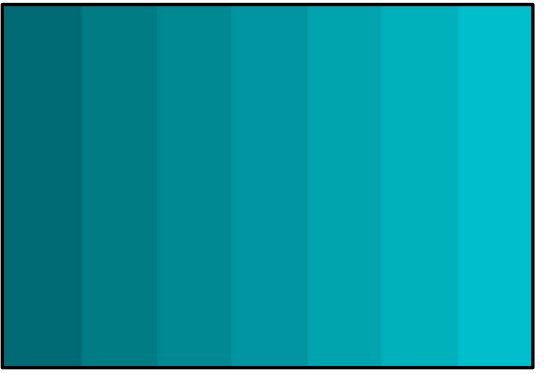

Low contrast, high saturation

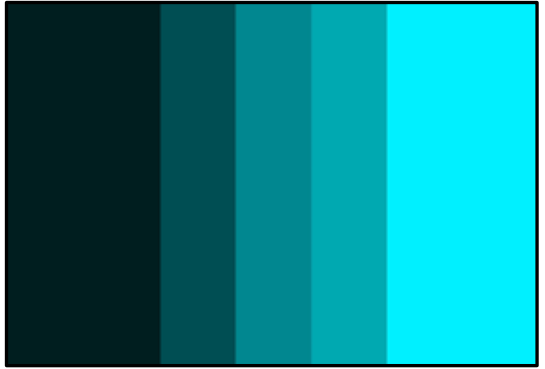

High contrast, high saturation 


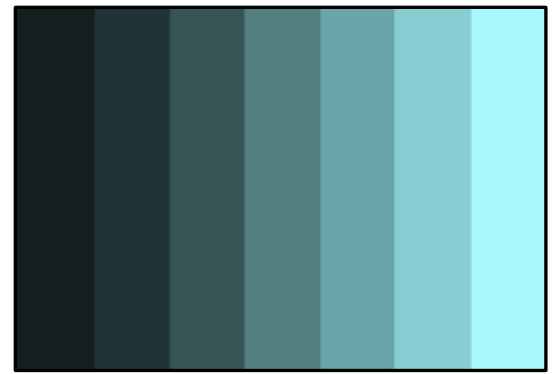

Normal contrast, high saturation

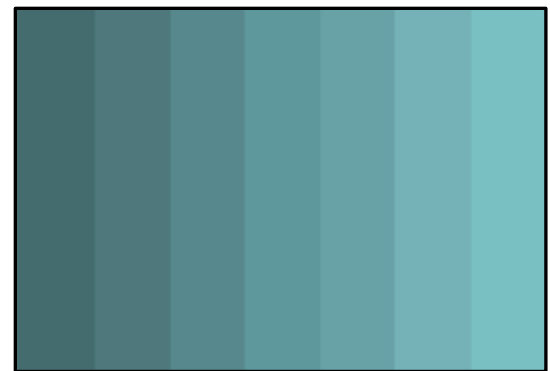

Low contrast, low saturation

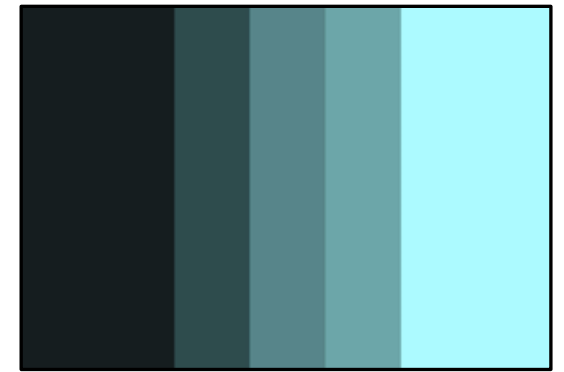

High contrast, low saturation

\section{SHARPNESS}

Sharpness refers to the clarity of an image at the pixel-level. Increasing the sharpness of an image increases the contrast between light and dark areas of pixels to create the impression of sharper lines and greater detail. If over-sharpened, an image can look grainy with light pixelated halos around areas of high contrast.

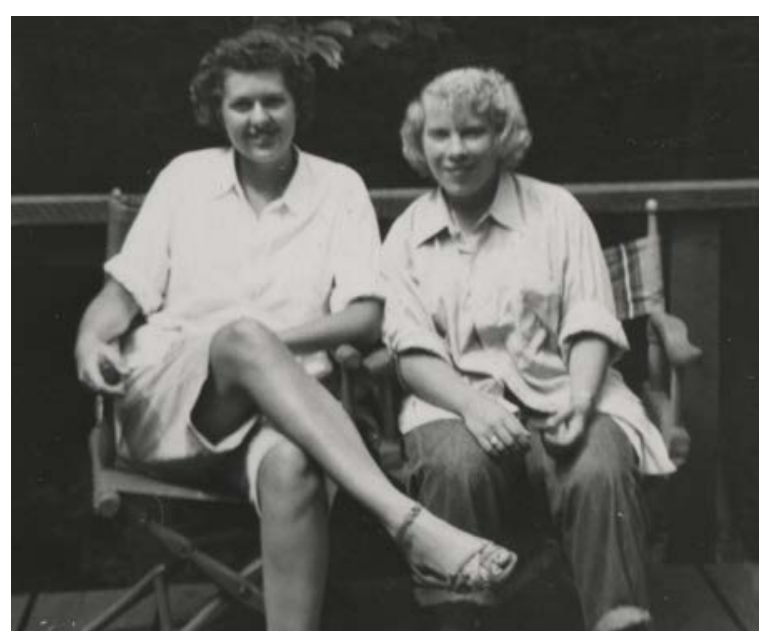

Original image

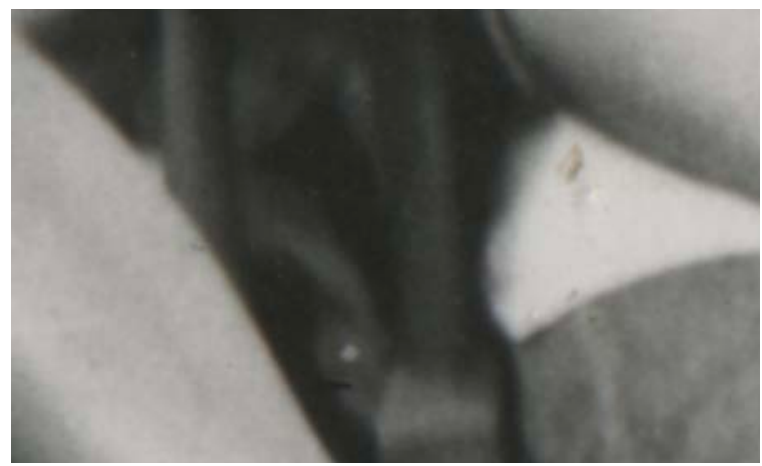

Original image, 500\% zoom

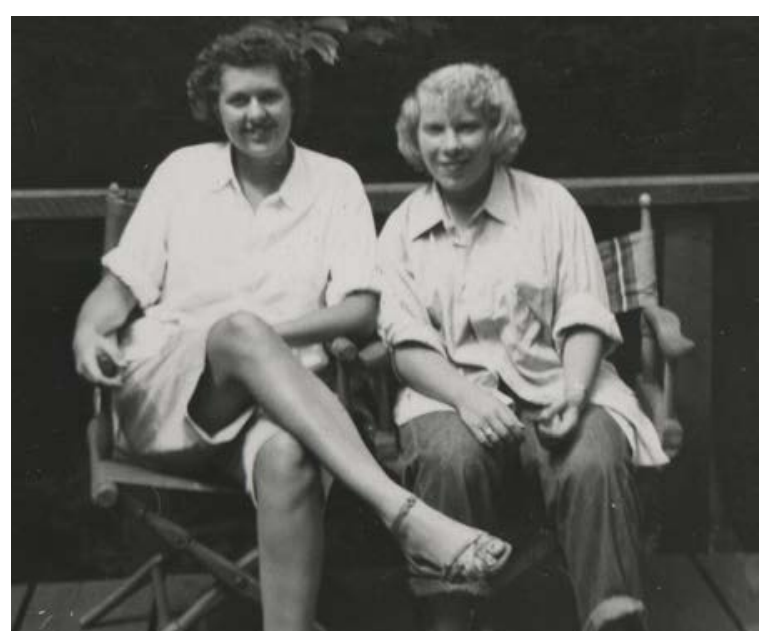

Sharpening filter applied in Photoshop

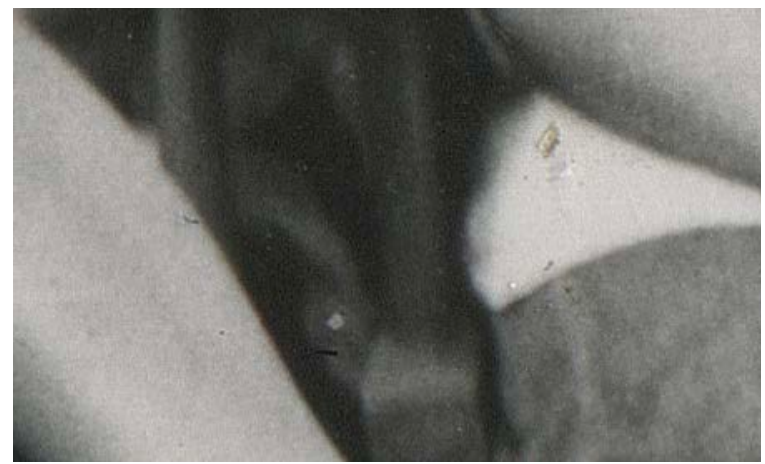

Sharpening filter applied in Photoshop, 500\% 


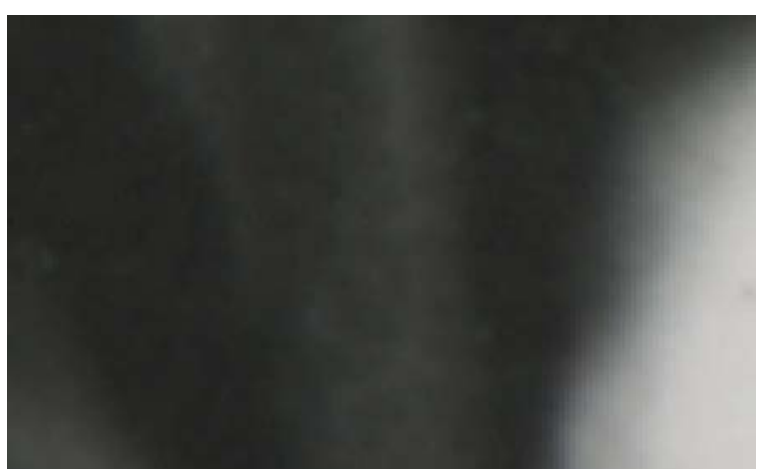

Original image, $800 \%$ zoom

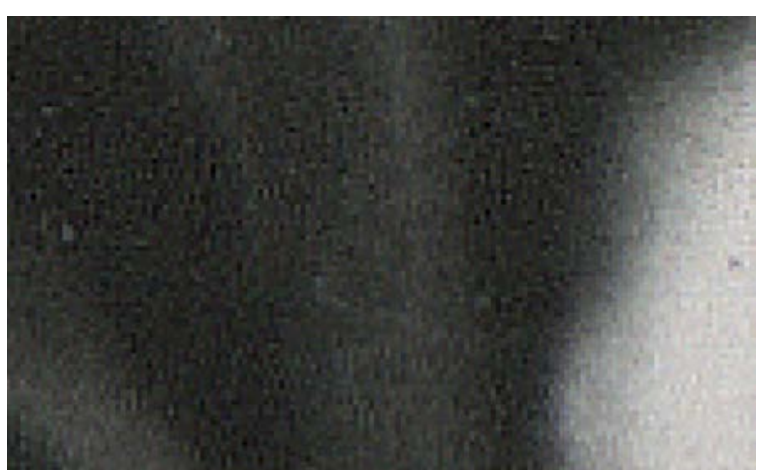

Sharpening filter applied in Photoshop, 800 


\subsection{Digitizing With a DSLR Camera and Copy Stand}

A great way to digitize reflective materials (papers, books, photographs, photography albums, small objects, etc.) is to use a digital camera, copy stand, and table top lights. Using a digital camera that is capable of shooting in a RAW file format (CR2, DNG, $\mathrm{NEF}$, etc.) will allow you to capture high resolution images very quickly.

\section{What You Will Need:}

- A digital camera ${ }^{44}$ (Canon 5Dmkll)

- An appropriate lens ${ }^{45}$ (Tamron 24-70mm f/2.8)

- Copy stand or tripod ${ }^{46}$

- 2 LED Lights ${ }^{47}$

- Colour checker card

- Mini USB to USB 3.0 cable (optional, to connect the camera to the computer)

- Cotton or nitrile gloves

- Computer (with a camera utility installed ${ }^{48}$ )

44. Digital Single Lens Reflex Cameras (DSLRs) such as the Canon Rebel or EOS series, the Nikon D series, or Pentax K series, or newer mirrorless cameras such as the Fujifilm $\mathrm{X}$ series, Panasonic Lumix series, or Sony Alpha series. The most important thing is that the camera be able to shoot in a RAW format. Cameras with interchangeable lenses allow for more flexibility when shooting, and are highly recommended.

45. A macro lens is recommended (although not strictly necessary) for copy stand work because it will allow you to focus on a range of objects that are very close to the camera. Good lenses for copy stand work include the Canon EF $100 \mathrm{~mm}$ f/2.8 Macro Lens, Canon $65 \mathrm{~mm}$ f/2.8 Macro Lens, Tamron SP $90 \mathrm{~mm}$ $\mathrm{f} / 2.8$ Macro Lens, and Sigma $70 \mathrm{~mm} \mathrm{f} / 2.8$ Macro Lens. Make sure that the lens will work with the brand and type of camera you are using; Third party companies like Tamron and Sigma manufacture lenses for both Canon and Nikon cameras, but they are not interchangeable. Additionally, some lenses will only work with cropped sensor cameras (APS-C) and others only with full-frame sensors. Cropped sensors are smaller than full-frame sensors.

46. The larger the documents you want to photograph, the larger your copy stand will need to be because the camera will need to be positioned further away from the document to fit it inside the camera frame. I am digitizing materials up to approximately $8.5 \times 11$ inches $(21.6 \times 27.9 \mathrm{~cm})$ and am using a Kaiser copy stand with a 23.25-inch $(59.1 \mathrm{~cm})$ column and $12.5 \times 15$ inch $(31.8 \times 38.1 \mathrm{~cm})$ baseboard. If using a tripod, it is important that the tripod arm be able to swing down to a horizontal angle to allow the camera to be parallel to the object being digitized. I find that copy stands tend to be easier to work with when digitizing archival material, and they are relatively inexpensive or can be built at home with basic tools.

47. Daylight temperature (between $5500 \mathrm{~K}$ and $6500 \mathrm{~K}$ ) preferable but not necessary. I am using 2 Polaroid High Output Super Bright LED Lights that came with a Polaroid Pro Table Top Photo Studio Kit I purchased for $\$ 100$. 
There are two ways you can use a copy stand with a digital camera. The first is to take photos, saving to the memory card, then remove the card and load the photos onto your computer. This method can be difficult if you are unable to look through the camera's viewfinder to focus, which will likely be the case if the camera is attached to the copy stand. The other method, which is preferred, is to tether the camera to the computer (connect the camera to the computer with a USB cable) so you can see what the camera is seeing on your computer, adjust the focus and capture settings, and fire shots directly from the EOS Utility Interface.

1. Place the copy stand on a clean, uncluttered, spacious surface such as a dining table or large desk.

2. Attach the camera to the copy stand by screwing it onto the camera mount using the tripod thread under the camera.

3. Use the locking screws on the holding arm to adjust the height of the camera.

4. Place one light on either side of the copy stand baseboard, positioning the heads at 45-degree angles.

5. Plug the mini USB cable into the camera and plug the other end into your computer.

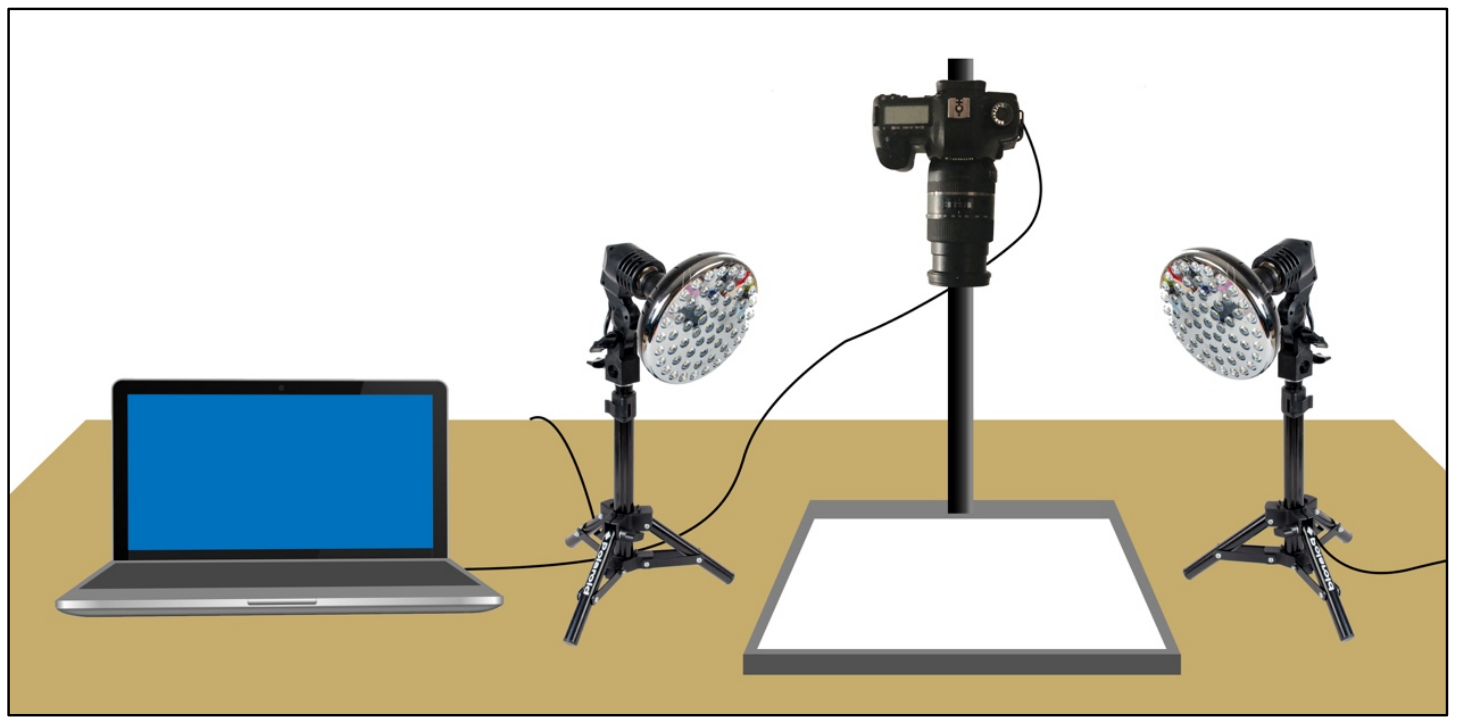

48. I used Canon's EOS Utility 2 program. 
6. Turn the camera ON.

7. Open the EOS UTILITY program, which you can download from Canon's website. Make sure to download the version that is compatible with both your camera model and operating system.

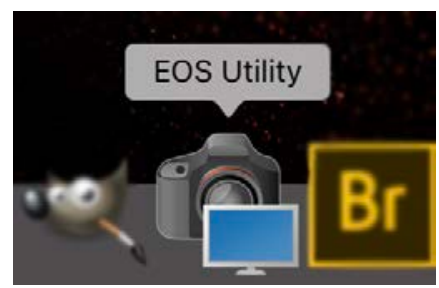

8. Next click CAMERA SETTINGS I REMOTE SHOOTING. If the option is grayed out, it means that the computer is not communicating with the camera. Try turning the camera off and on again or unplugging it from the computer, waiting several seconds, and then plugging it back in.

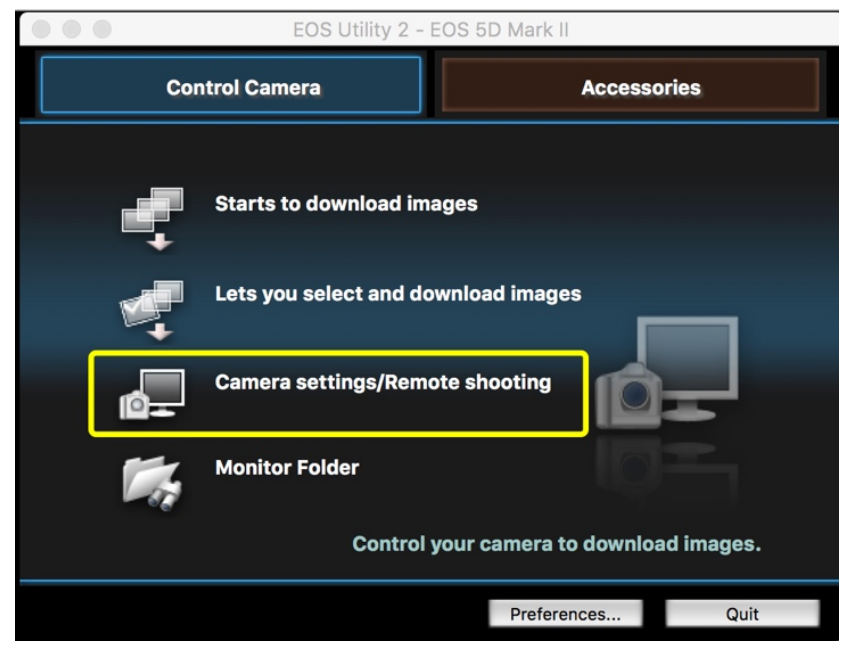

9. The remote shooting window will now appear.

10. To change the aperture, shutter speed, and ISO, double click on each of the settings in the remote shooting window and use the arrow buttons to adjust.

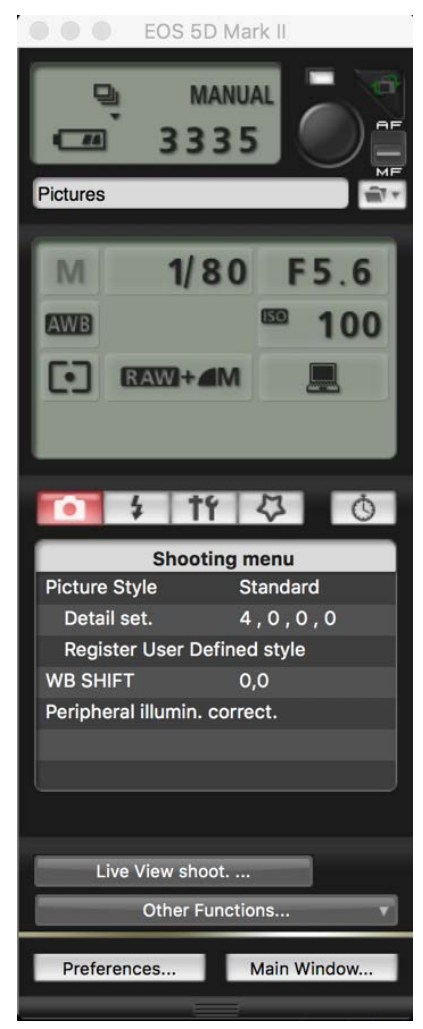

The settings you should use depend on the type of lights and lens you are using. I start with an aperture of about $f / 8$ or $f / 5.6$, a 
low ISO like 100 to minimize noise from the camera sensor, and whatever shutter speed is necessary to produce a good exposure. Because the camera is attached to the copy stand, it is possible to use slow shutter speeds, lower than 1/60 second which is the slowest that can be held by hand.

11. Next, click PREFERENCES at the bottom of the remote shooting window.

Preferences...

12. In the window that appears, click the drop-down menu in the upper left corner. Click DESTINATION FOLDER.

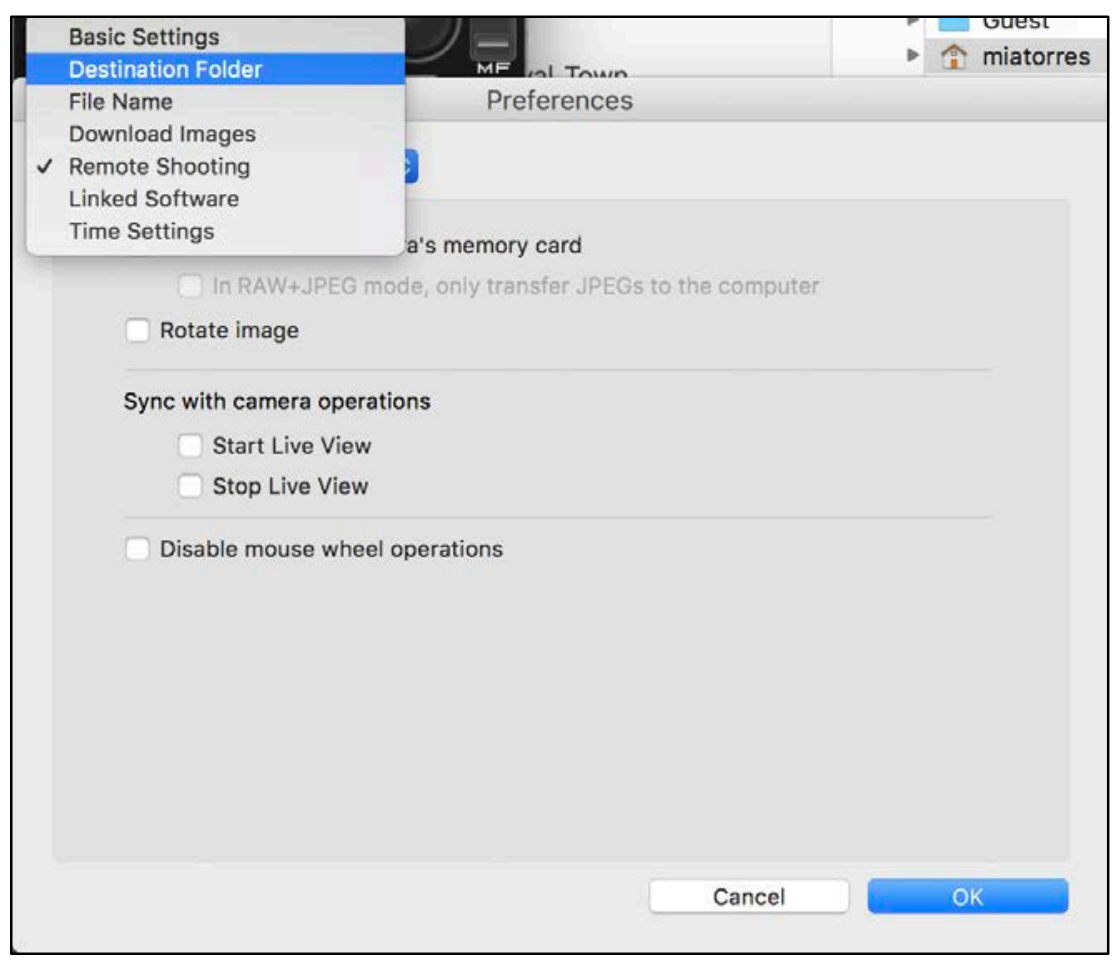

13. Make a new folder within your main archiving folder and name it appropriately.

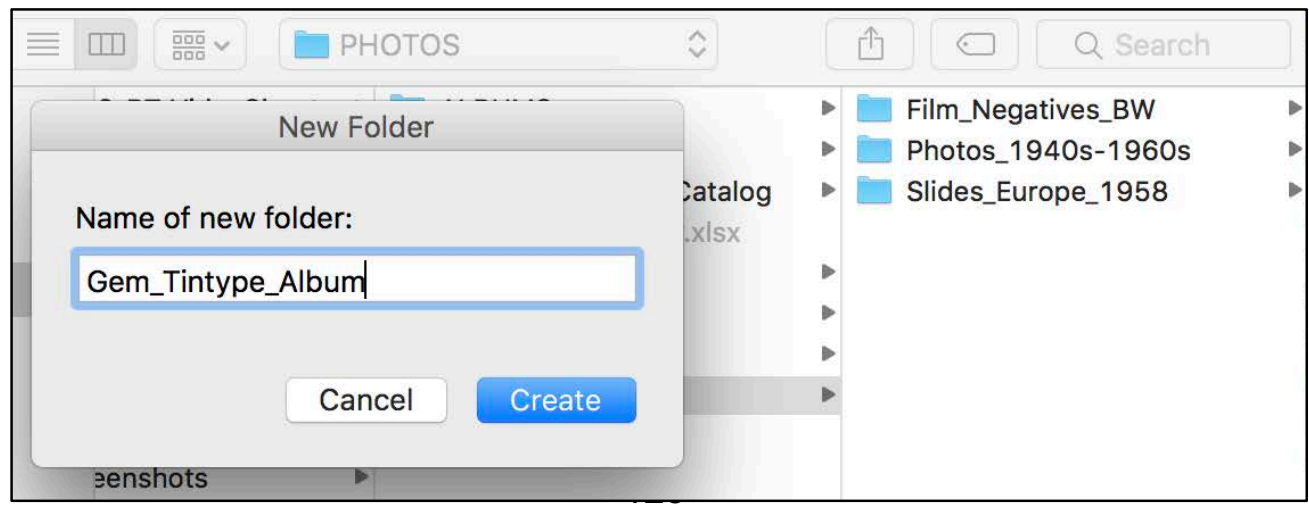


14. Within that folder, create another folder called ORIGINAL_RAW. This is where all of the photographs taken using the copy stand setup will be saved. Once you are done shooting, you will import the photographs into Lightroom, process them, and then export them as TIFFs to a folder called MASTER_TIFF, just as you would with scanned images.

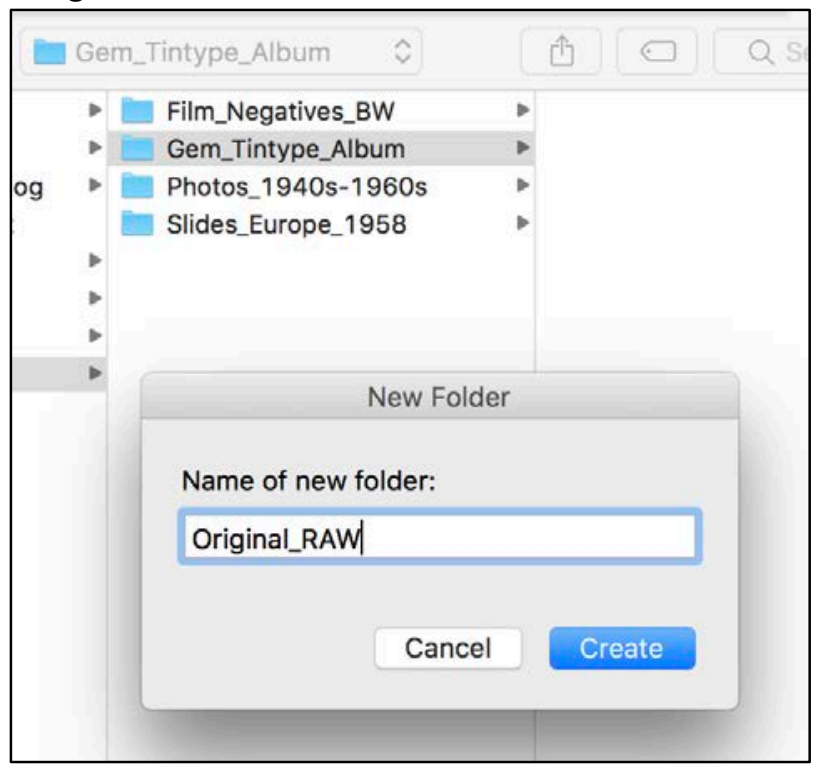

15. To see what the camera is seeing in real time, click LIVE VIEW SHOOT... at the bottom of the remote shooting window.

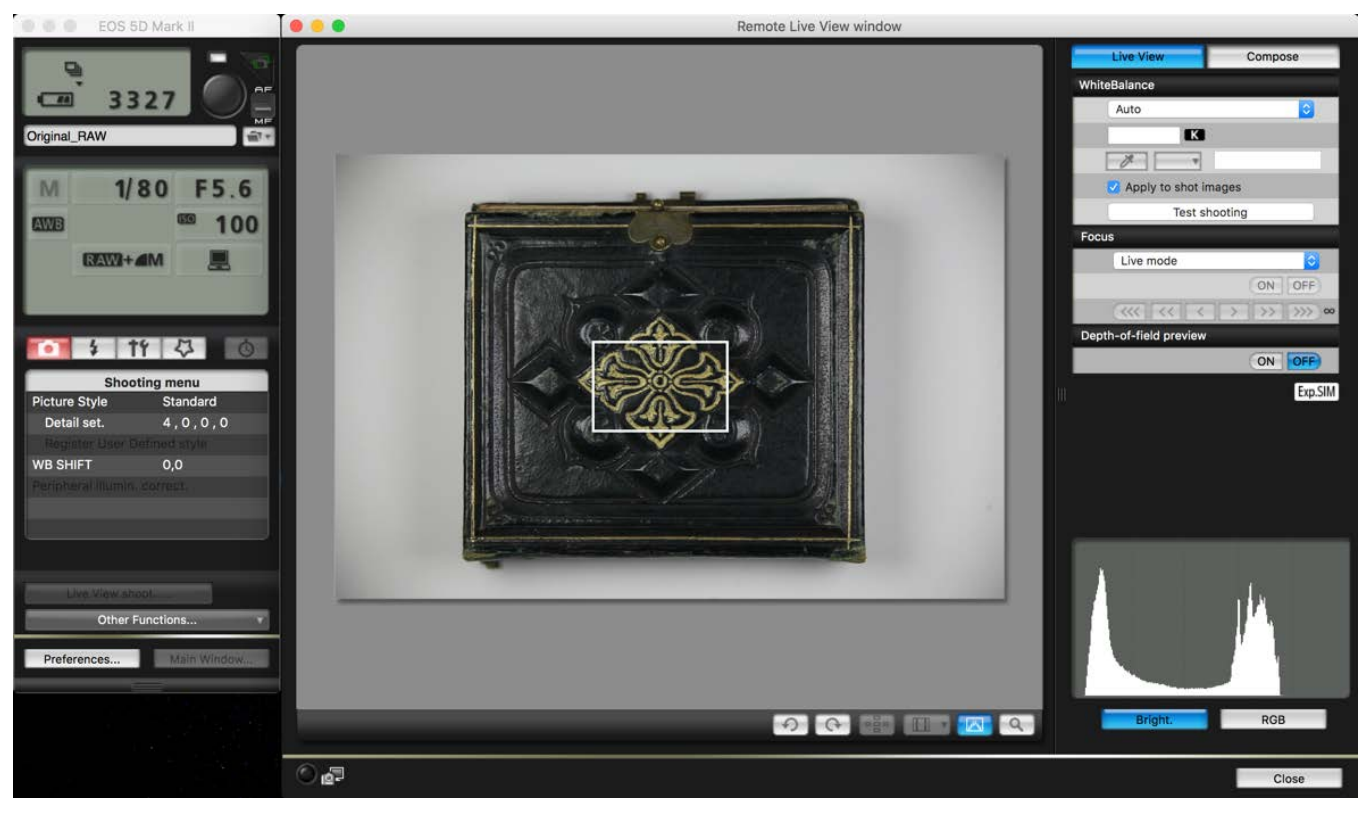


16. Position the item you are going to photograph in the camera frame. Because the EOS Utility program is set to Live View mode, you will be able to see your hands moving the item around on the computer screen. If necessary lower the camera or zoom in with the lens to fill in the majority of the image frame with the object. The larger an object is in the frame the higher resolution it will be and the more detail you will be able to capture.
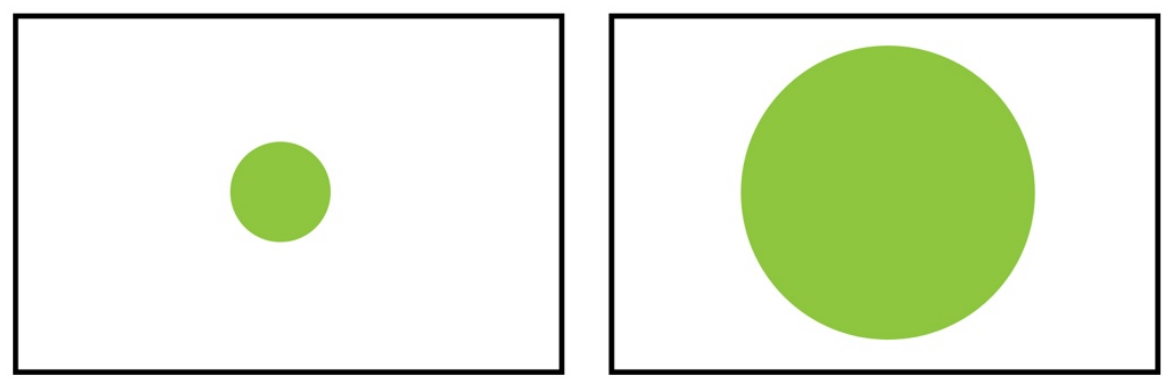

17. To check the camera's focus, click the MAGNIFYING GLASS icon in the lower right area of the Live View window. The ZOOM VIEW window will now appear.
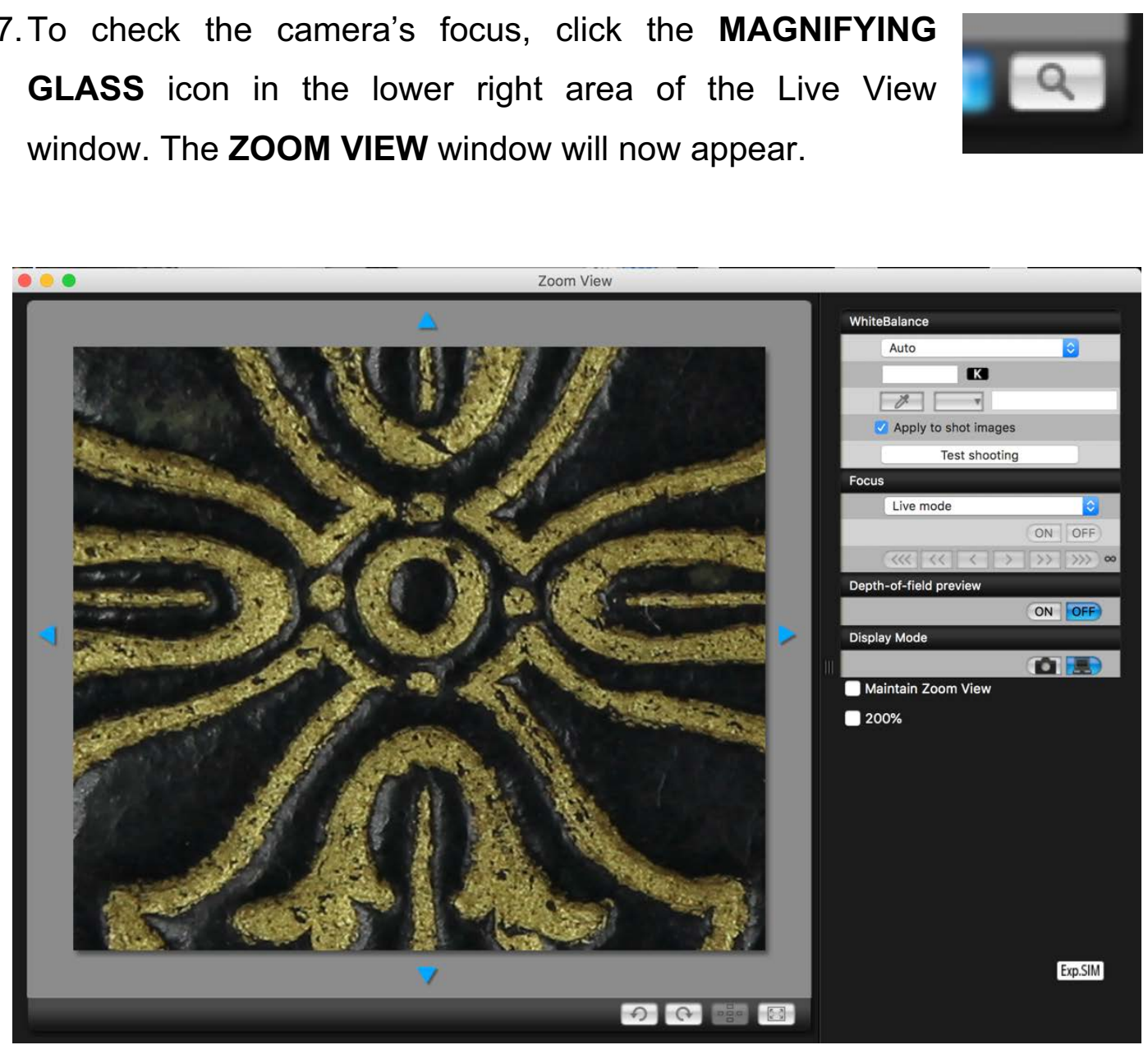
18. Use the focusing ring on the lens to manually adjust the focus. Make sure the focus is set to manual, not automatic. There is usually a switch on the lens itself.

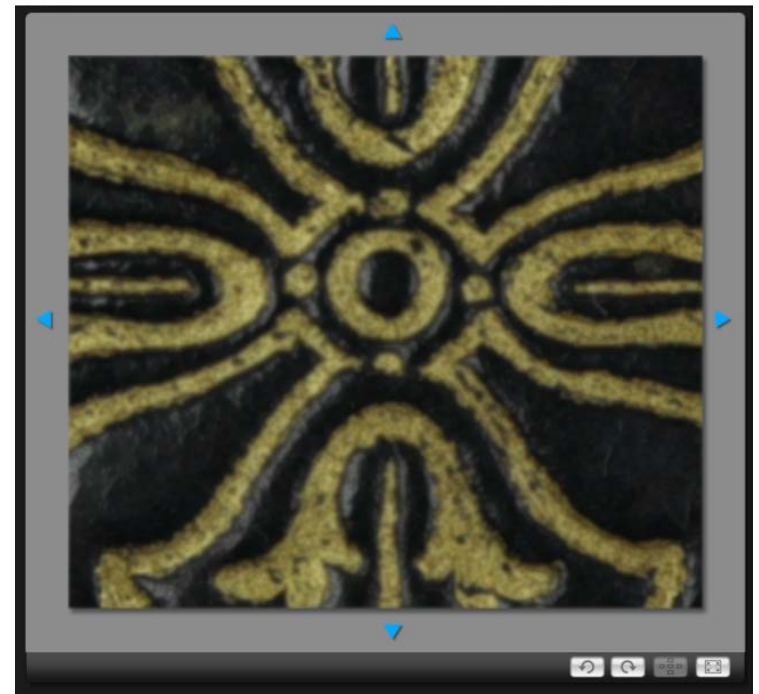

Out of focus

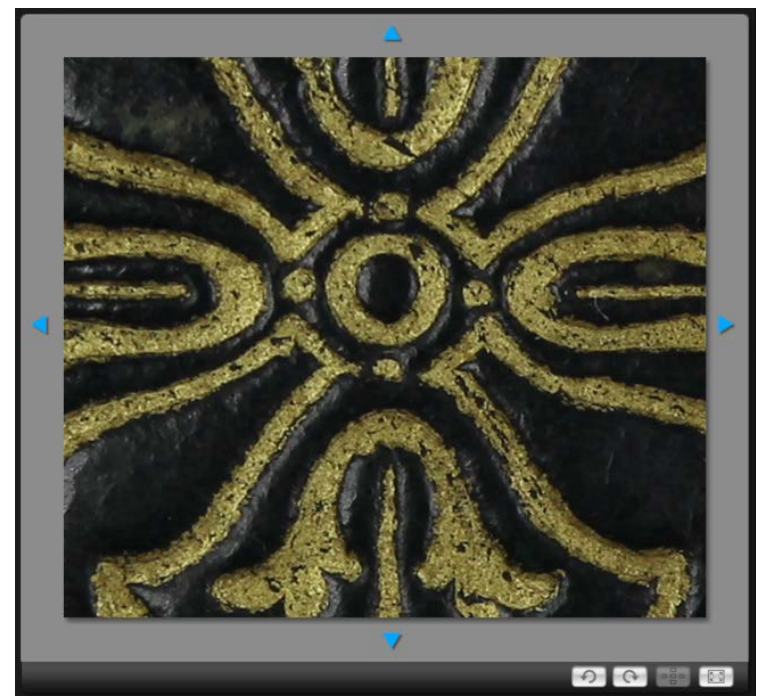

In focus

19. After you have completed the camera set up, place a small ruler or colour checker card along an edge of the object. This will provide a size reference as well as reference colours to use when white balancing during post-processing.

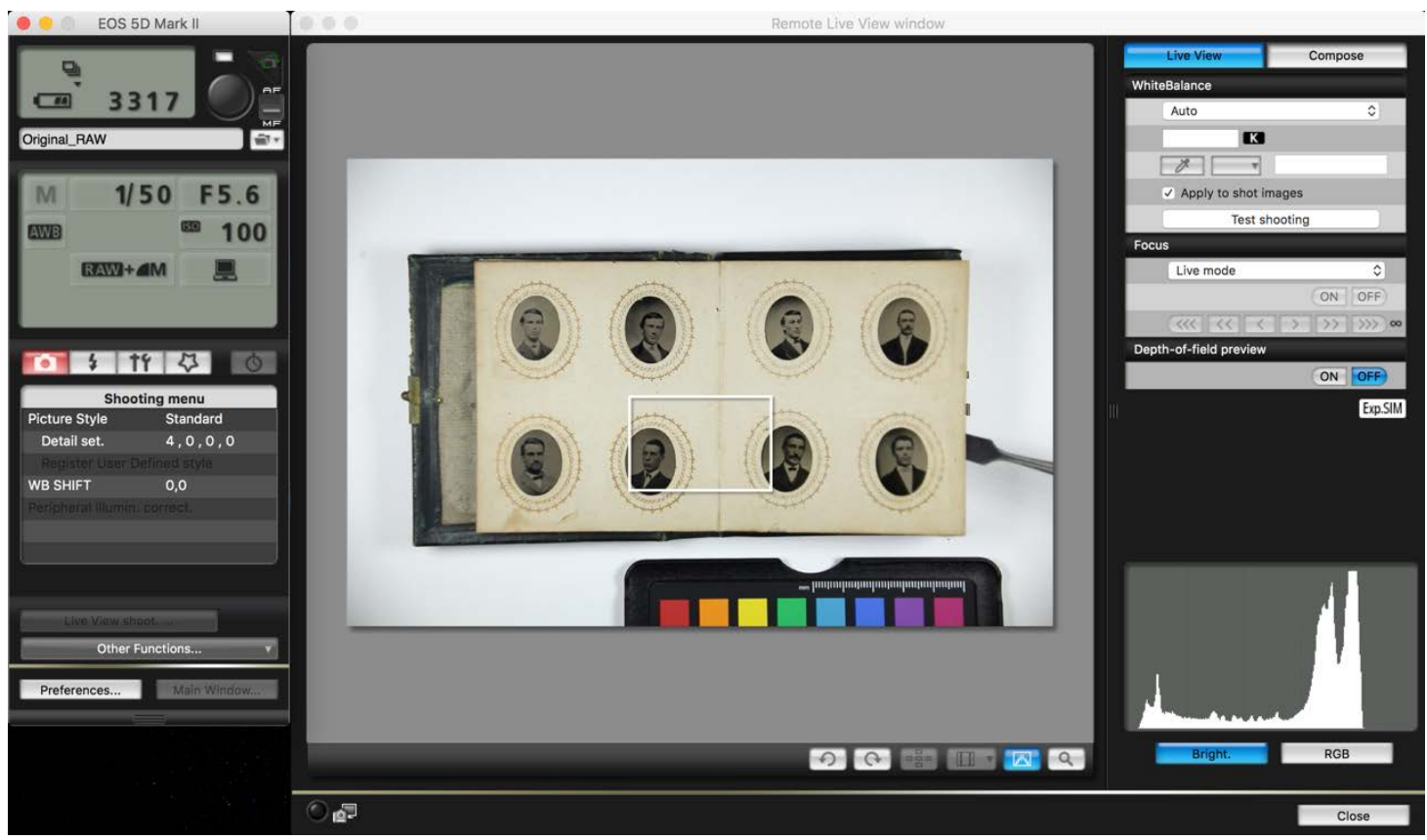


20. To ensure that you are not over or under exposing the photographs, make sure that neither of the edges of the histogram in the lower right area of the Remote Live View window are clipped by the edges of the histogram. This would indicate that either the shadows are too dark or the highlights are too bright.

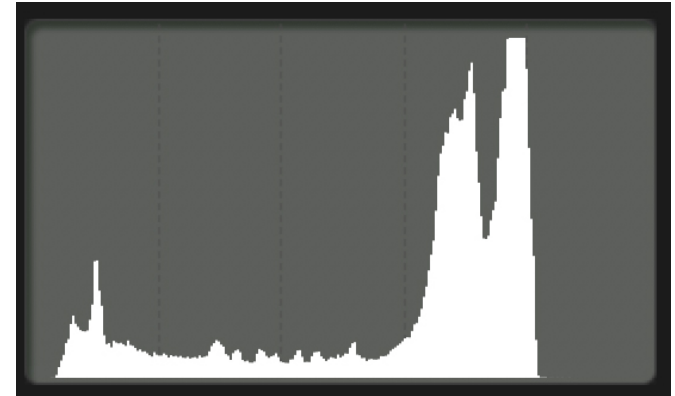

Good exposure

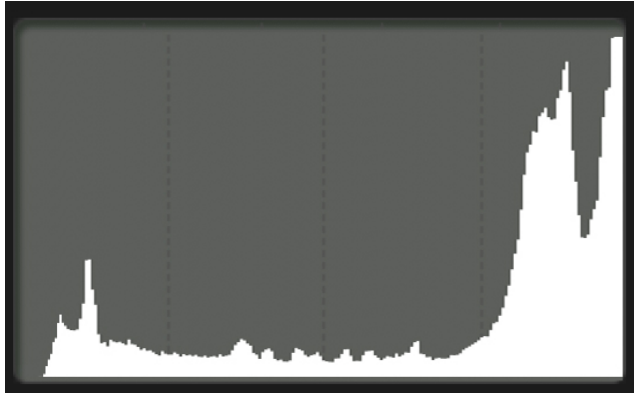

Too bright

21. To capture the image, click the round button at the top of the Remote Shooting window.

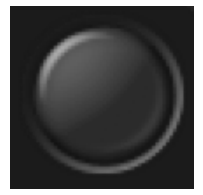

22. Once you have finished capturing the images, continue on to Section 3: Postprocessing.

\begin{tabular}{|c|c|c|c|c|c|c|c|c|}
\hline ALBUMS & $\rightarrow$ & Gem_Tintype_Album & $\Rightarrow$ & Access_JPEG & $\rightarrow$ & 區 & KAUFMAN_A...intype_01.jpg & \\
\hline BOUND DOCS & D & Japan_Album & D & Master_TIFF & - & 圆 & KAUFMAN_A...intype_02.jpg & \\
\hline Kaufman_Archive_Catalog & - & Peru_Album & - & Original_RAW & - & $\underline{m}$ & KAUFMAN_A...intype_03.jpg & \\
\hline 凶] Kaufman_Inventory.xIsx & & & & & & 토 & KAUFMAN_A...intype_04.jpg & \\
\hline LOOSE DOCS & • & & & & & 囸 & KAUFMAN_A...intype_05.jpg & \\
\hline METADATA & - & & & & & 槧 & KAUFMAN_A...intype_06.jpg & \\
\hline OBJECTS & D & & & & & 監 & KAUFMAN_A...intype_07.jpg & \\
\hline PHOTOS & $\triangleright$ & & & & & 些 & KAUFMAN_A_...intype_08.jpg & \\
\hline & & & & & & & KAUFMAN_A...intype_09.jpg & \\
\hline & & & & & & & KAUFMAN_A...intype_10.jpg & KAUFMAN_Album_Gem \\
\hline & & & & & & & $\begin{array}{l}\text { KAUFMAN_A...intype_11.jpg } \\
\text { KAUFMAN_A...intype } 12 . j p g\end{array}$ & Tintype_01.jpg \\
\hline & & & & & & & KAUFMAN_A....intype_13.jpg & $\begin{array}{ll} & \text { JPEG - 8.3 MB } \\
\text { Created } 2018-06-28\end{array}$ \\
\hline & & & & & & & KAUFMAN_A...intype_14.jpg & Modified $2018-06-28$ \\
\hline & & & & & & & KAUFMAN_A...intype_15.jpg & Last opened -- \\
\hline & & & & & & & KAUFMAN_A...intype_16.jpg & Dimensions $3700 \times 3898$ \\
\hline & & & & & & & & Add Tags... \\
\hline
\end{tabular}




\section{NOTES:}

- You may notice that there is a vignette around the photographs above. This is caused by the way the lens interacts with the lights. It can be removed very easily in Lightroom with the Lens Corrections > Enable Profile Corrections tool.

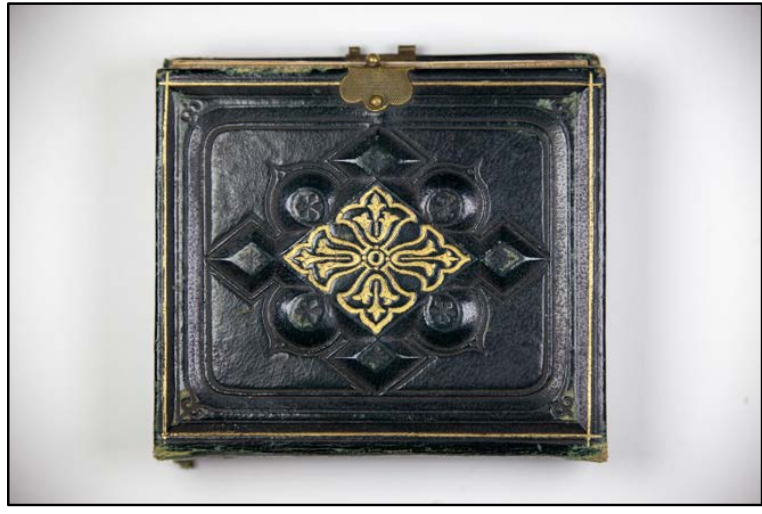

Before lens profile correction in Lightroom

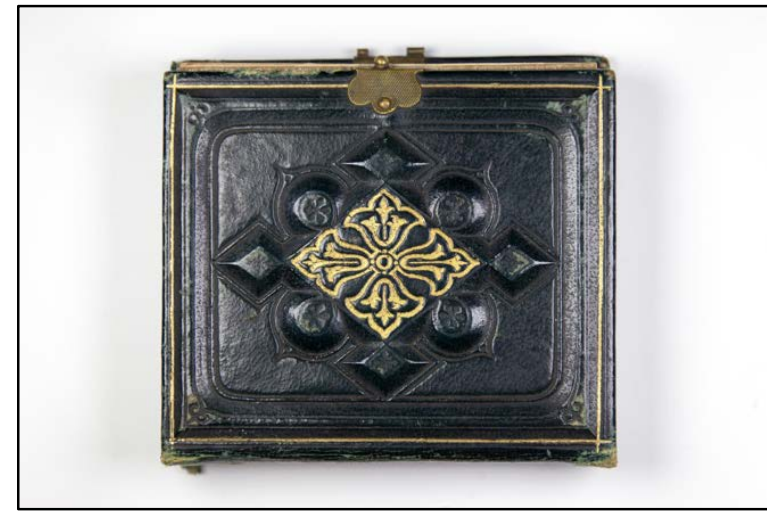

After lens profile correction in Lightroom

- You will need to rename the files in Lightroom because they will initially be saved with a camera-generated file name.

- For delicate bound objects, make sure to support the front and back cover when the item is open. This will ensure that no extra stress is placed on the spine and binding during digitization. I used a clean rolled-up microfiber cloth to support the front and back covers when digitizing the front pages and back pages of the album, when it could not sit flat. 


\subsection{Digitizing Small Objects}

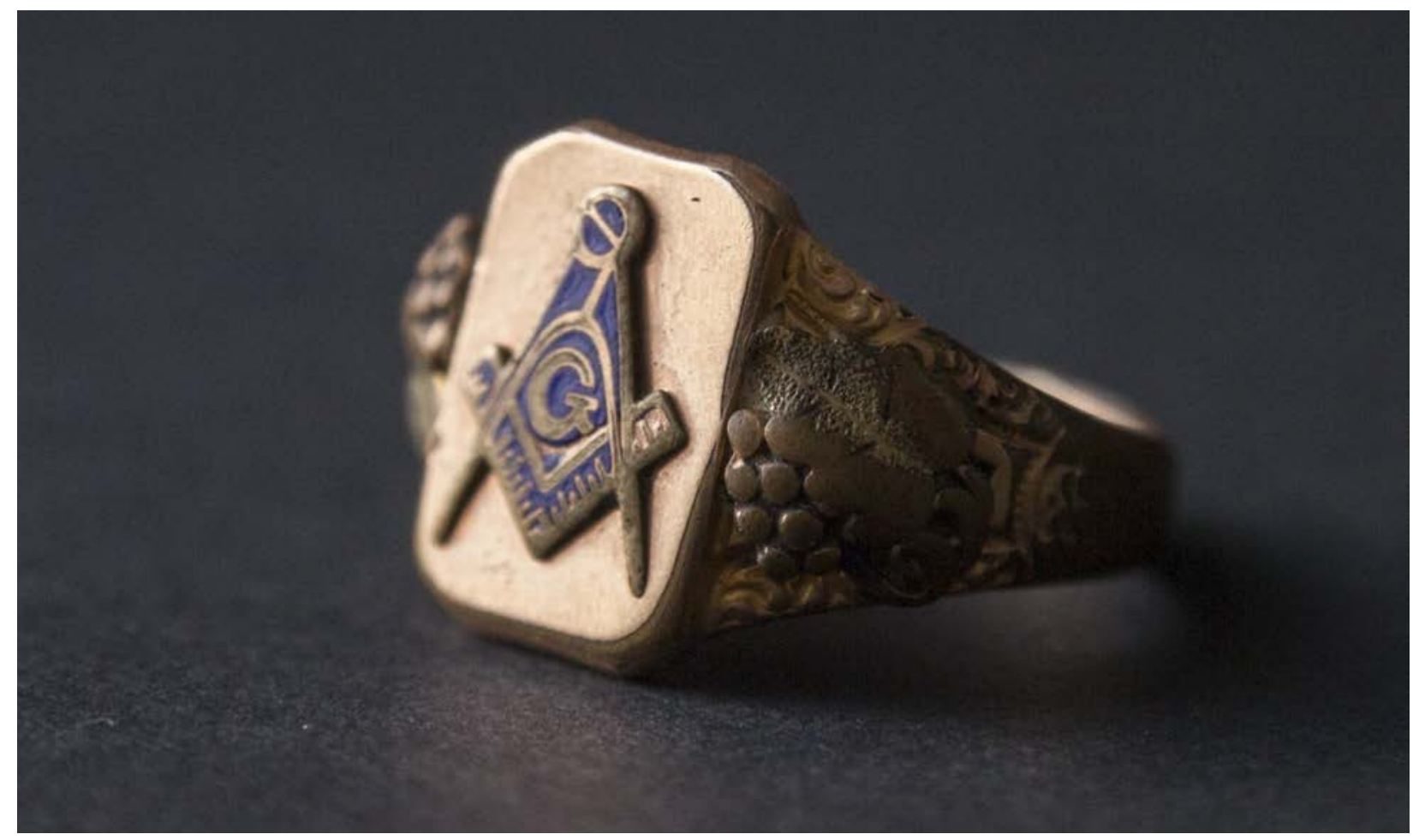

Many collections consist of more than just two-dimensional items; often they contain small objects, such as pins, medals, plaques, jewelry, nick knacks, small articles of clothing, personal accoutrement, small books, etc. While these kinds of items can't be digitized in the same way as a letter or photograph might be, it is still possible to create a high-quality digital record of each item. Although digital images of a three-dimensional object do not reflect the physicality of the object in the same way a scan of a letter or photograph might ${ }^{49}$, I believe that having a visual digital record is important for several reasons: If ever the original object were to be lost or damage, having high quality

49. There is a lot of academic and philosophical debate as to the faithfulness of a digital copy of analog objects and what might be lost in this translation from a physical mass with texture and weight to an imperceptible string of ones and zeros. As technology improves it may be possible to decrease the gap between a physical item and its digital surrogate, with the constantly increasing capabilities of optical sensors and digital imaging software, but some argue that no matter how 'real' a digital surrogate looks (some argue they are becoming hyper-real, making it possible to see detail that one would not be able to see with the naked eye), there will always be differences between what you can touch in your hands and what you can only see on a screen, the characteristic of physical tangibility being the most obvious. 
images of the item is important both for sentimental and financial reasons (like insurance claims). Additionally, if a family member or researcher wants to see or study the object, but you do not want to (or can't) send it to them, sharing digital photographs is an easier and risk-free option. I have no doubt that in the future it will become routine to scan objects and create 3D digital renderings at home (and perhaps even print 3D copies of grandma's costume jewelry!), but for the time being, taking good quality, welllit photographs of the objects is your best option.

As I was going through my family's collection, I found several small items of my grandfather's, who was a Freemason-a ring, a pendant, and what appears to be a cufflink, all with Masonic symbols. I knew I wanted to take photographs of the items, but I also knew that simply using ambient living room light and my well-used cell phone would not give me the high quality results I wanted.
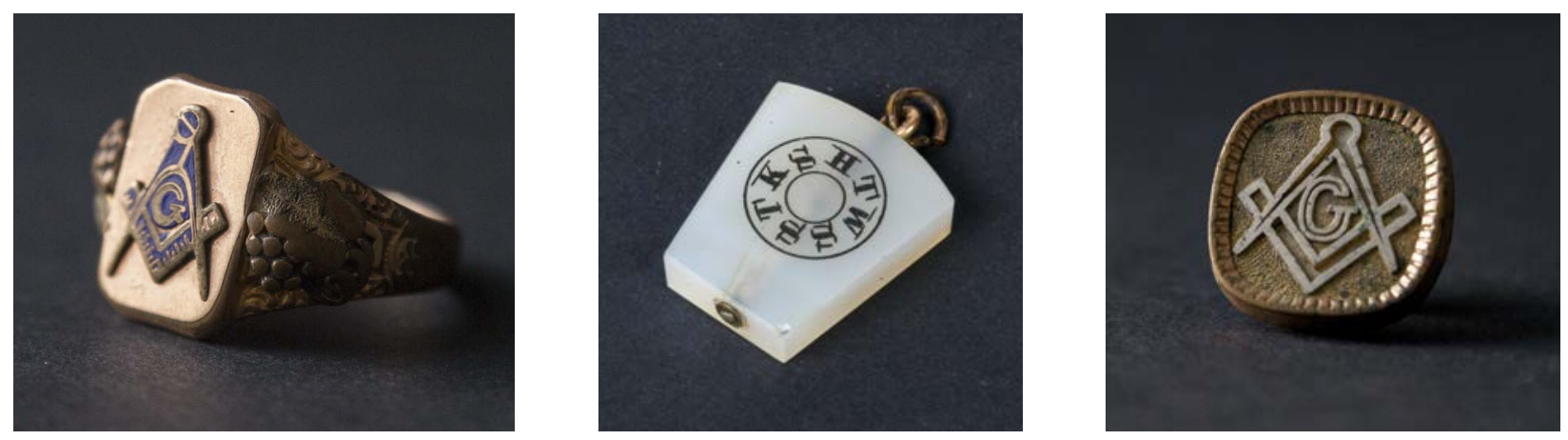


\section{DIGITAL CAMERA \& NATURAL LIGHT}

If you do not have access to lights, it is possible to photograph small objects with a digital camera in a well-lit space. Natural light is not as bright or consistent as studio so the exposures will be longer necessitating the use of a tripod.

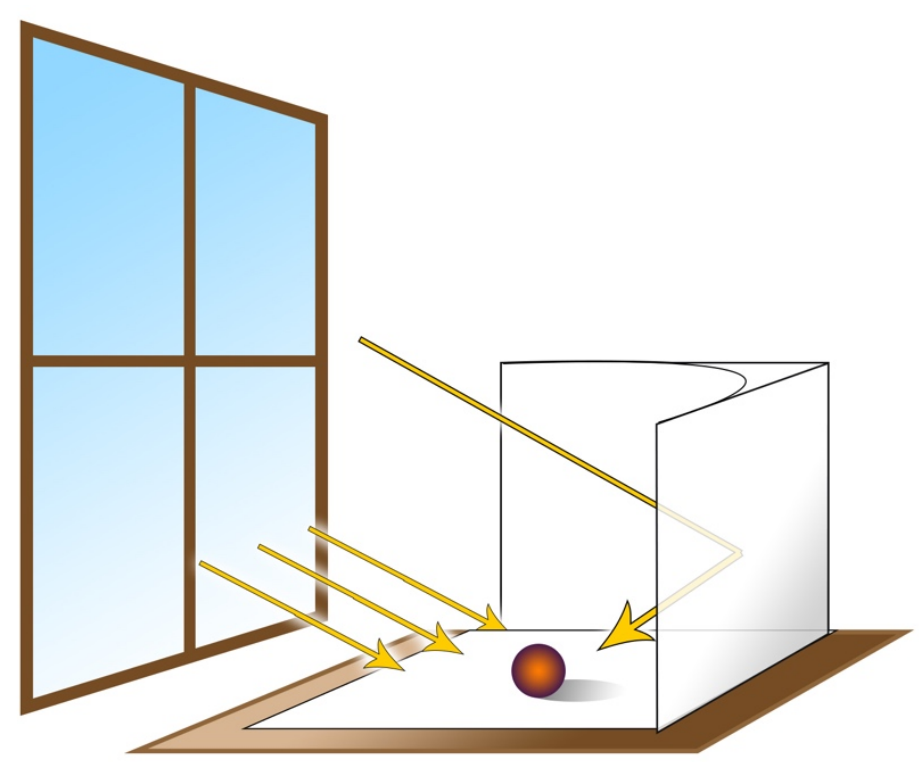

\section{What you will need:}

- A clean, portable working space or one close to a large window.

- Access to a large window.

- White paper (black or grey paper is also acceptable)

- Digital camera

- Tripod

- Colour checker card

- Ruler 
1. Begin by placing a piece of white paper on a clean surface close to a large window.

2. Place two additional pieces of paper, or one longer, curved piece of paper opposite the window. This will help bounce light back onto the object you are photographing.

3. Attach the camera to the tripod. You can either shoot to the memory card in the camera, or you can tether the camera to a computer, as described in the previous section.

4. Place the colour checker card on the white paper. This will allow you to ensure that the white balance is correct in post-production.

5. Adjust the tripod so that the objects fills the frame as much as possible, still keeping it in sharp focus.

6. Make sure not to use an ISO greater than 200 on your camera. The higher the ISO, the grainier the image.

7. Set the aperture to two stops above the smallest aperture..$^{50}$ Using a larger aperture, such as $f / 1.2$ or $f / 2.8$ will decrease the depth of field causing the focus plan to be much shallower. This can become problematic when shooting 3D objects as they have more dimensional space than a flat object.

50. FADGI guidelines state that the best aperture to use is the one that is two stops larger than smallest aperture the available on the lens. If the lens maximum is $f / 22$, digitization should be done with an $f / 11$ aperture $(f / 22, f / 16, f / 11)$. If the maximum aperture is $f / 32$, digitization should be done at $f / 16(f / 32$, $f / 22, f / 16)$. Larger numbers indicate smaller apertures. The aperture number is determined by the number of aperture diameters that can fit between the lens and the film or camera sensor (focal length $\div$ aperture diameter $=$ aperture value) . Each f-stop allows either $2 x$ or $1 / 2 x$ the amount of light, when opening or closing the aperture, respectively. 
8. Photograph the colour checker card on the white paper. When you are satisfied with the exposure, remove the colour checker card and place the archival object on the paper where the colour checker card was.

9. Place a ruler in the frame next to the object. If possible, prop the ruler into a vertical position to provide as accurate a size reference as possible.

10. Photograph the object, carefully rotating it 90 degrees for each shot. You may also want to take a photograph looking directly down onto the object, but be sure not to drop the camera onto the object!

11. After you have photograph the object, continue on to Section 3: Post-Processing

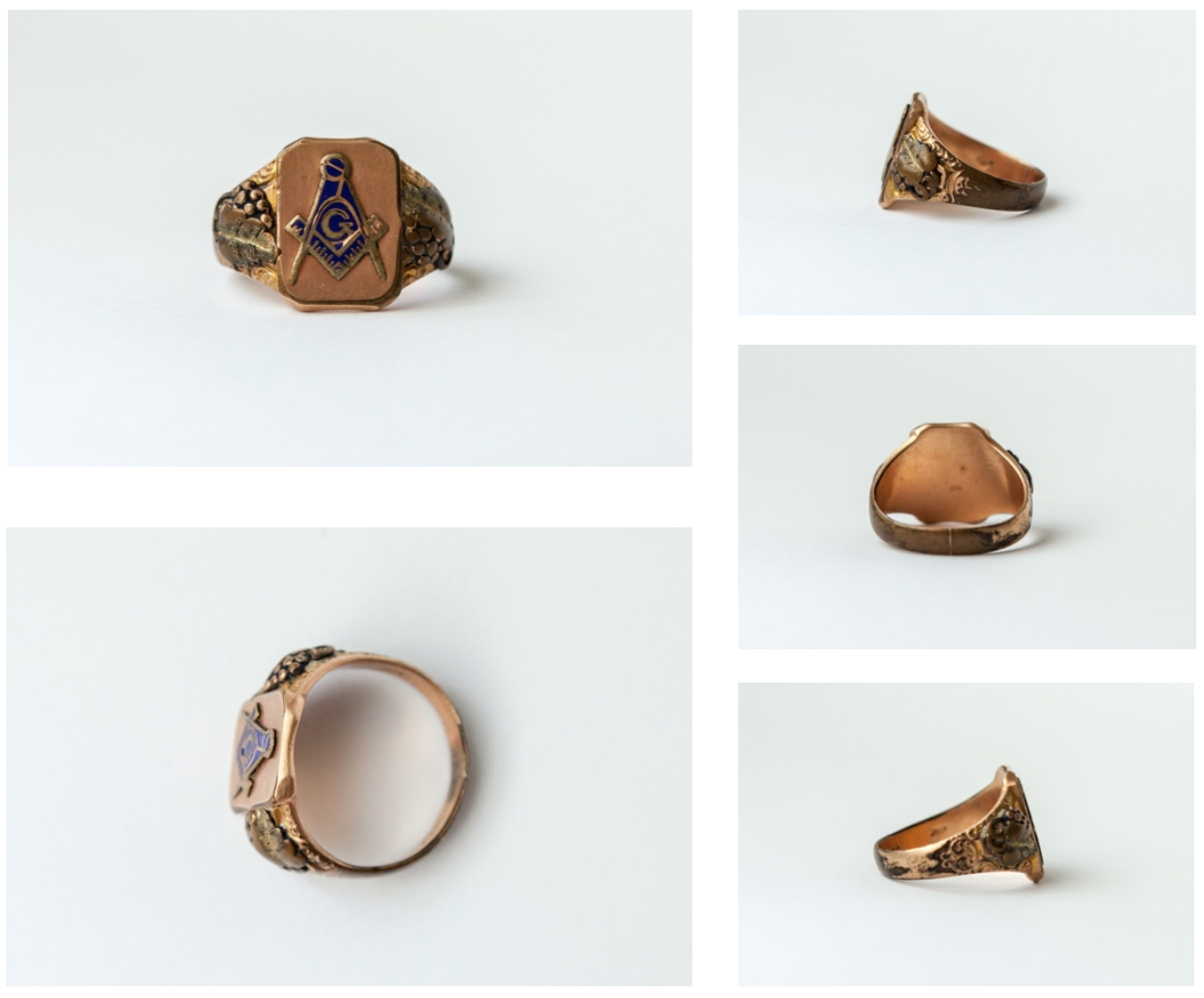

Raw images shot with natural light 


\section{DIGITAL CAMERA, STUDIO LIGHTS, \& COMPOSITING LIGHTBOX}

Many objects have reflective surfaces but still need to be lit in order to be photographed. Photographing the objects with lights causes intrusive glares that obscure large portions of the object, but the object is too dark when the lights are turned off. In cases like these, you may want to consider compositing two images that have each been lit by opposite lights, and then combining them to create a final image with no glare.

Photograph the object with the light on, and then, carefully so as not to move the object or the camera, turn off the left light and turn on the right light. Take a second picture, and then open both in Photoshop.
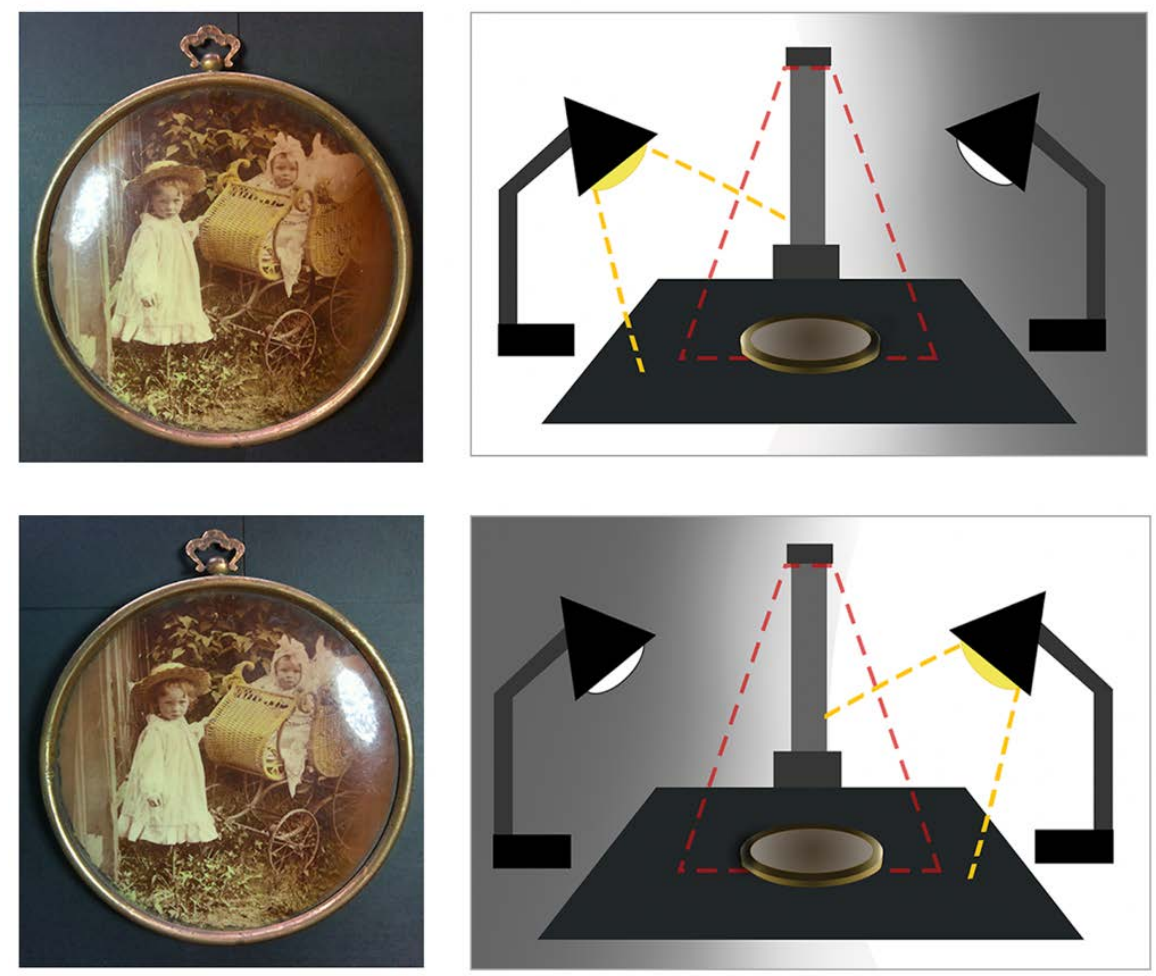

Drag one exposure onto the other and lower the opacity of the layer to around $40 \%$. This will allow you to see both layers in order to properly align the two. Once the two layers have been registered, increase the opacity the top layer back to $100 \%$. Use the eraser tool, sized at around 500 pixels with a hardness of roughly $75 \%$, to erase the glare side of the top layer to expose the bottom layer. 


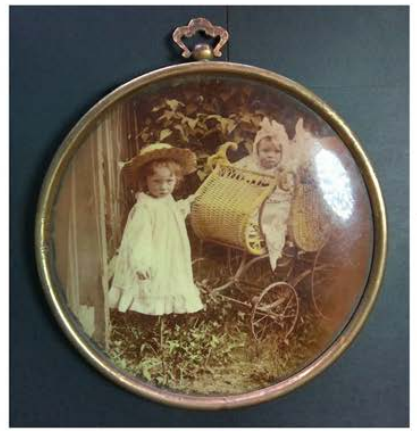

Bottom layer

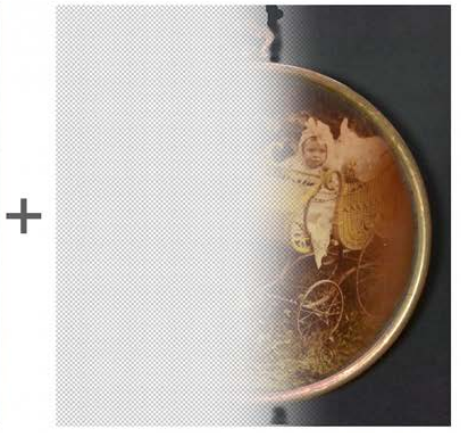

Top Layer, with half erased

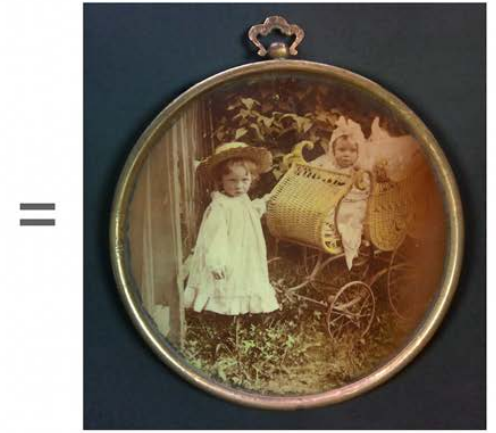

Final composited image.

The images shown above were taken using the HoverCam Solo 8Plus, but the principle is the same when using a digital camera.

\section{DIGITAL CAMERA, STUDIO LIGHTS, \& LIGHTBOX}

Another way to photograph shiny or reflective objects is to use a lightbox. A lightbox is usually a fabric or plastic square tent-like object that sits on a table top. It may have built-in lights or can be used with studio lights placed on either side. The idea is to use the semi-transparent sides of the box to soften or diffuse the light, preventing harsh highlights and reflections from you and your camera. This is a technique that is often used in commercial and product photography. This process combines steps used in this section as well as Section 2.8.

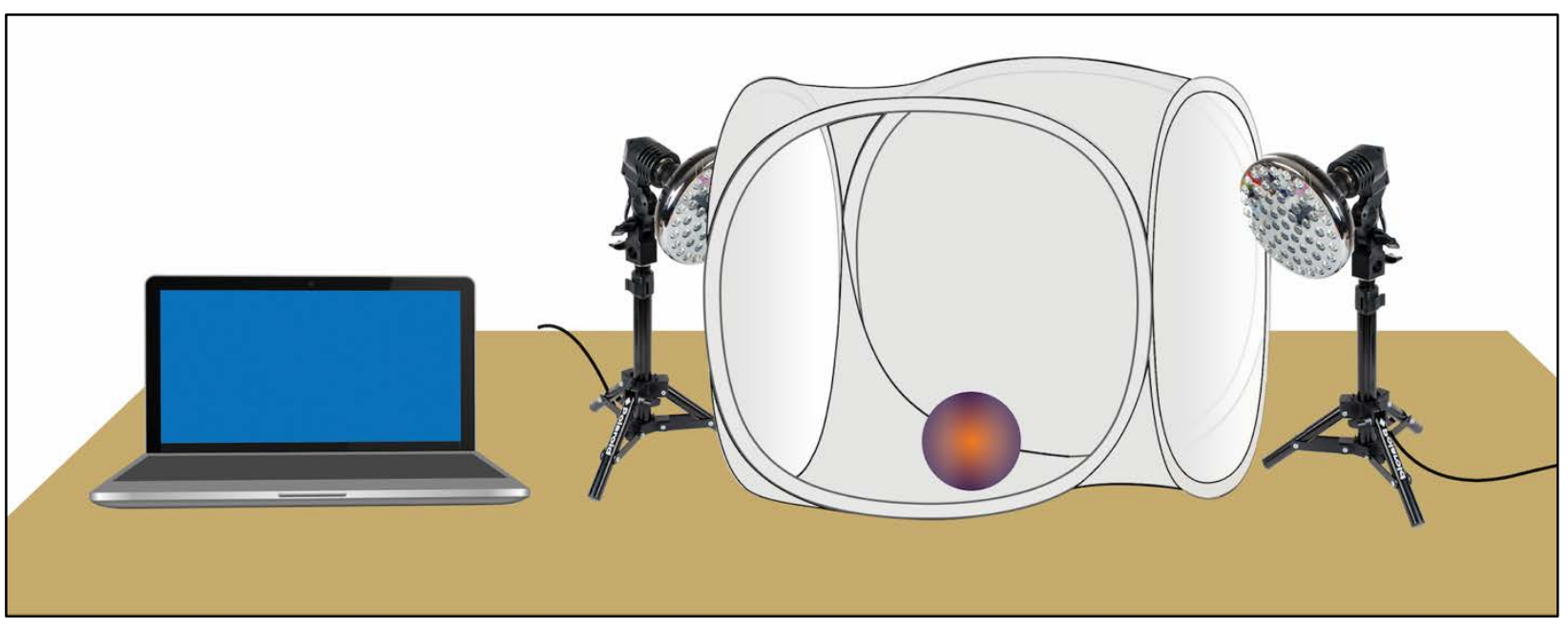


SECTION 3: POST-PROCESSING 


\subsection{Introduction To Post-Processing}

After digitizing your documents, you may want to do some post-processing to the files, whether it be cropping, de-skewing (rotating to straighten the image), adjusting the white balance, exposure, etc. FADGI ${ }^{51}$ guidelines do not allow for much post-processing of archival images, but they do advise that some basic post-processing may be used to create the most accurate digital representation possible I believe that complete adherence to these guidelines is impractical for small institutions and individuals who have limited resources, particularly in regard to FADGI's policies on the post-processing of digital files. ${ }^{52}$ For example, it will save a lot of time and money to scan an item and then de-skew later if needed, rather than rescan the document over and over until it is perfectly aligned. If the white balance and exposure of a digital photograph (of an archival object for example) are slightly off-perhaps too cool and a bit too darkadjusting these in post-processing will not drastically shorten the lifespan of your digital file. Yes, you will lose some pixel data ${ }^{53}$, but only a minute amount, certainly much less than perceivable to the naked eye. That being said, it is important to make sure that your scans and photographs are as close as possible to the correct exposure, colour, etc. when digitizing. The use of a colour checker card or grey card when taking digital photographs and certain level-measuring tools in scanning programs will help you to ensure you have a good exposure. I will review these methods in this chapter, with some elaboration in the appendices.

51. FADGI (Federal Agencies Digitization Guidelines Initiation) is a US governmental initiative to establish guidelines for the proper digitization of archival materials. For more information about FADGI, please refer to section 4.1 in the appendices.

52. FADGI guidelines only permit de-skewing in 90-degree increments, the reason being that because pixels are square, rotating them 45-degrees for example will force the computer to interpolate pixel data to fill in the voids created by the rotated pixel. Because this data is generated by the computer program and not in-camera or by a scanner, it will be inherently less accurate and less faithful to the original document.

53. "DICE Evaluation Parameters," Technical Guidelines for Digitizing Cultural Heritage Materials, The Creation of Raster Image Files, Federal Agencies Digital Guidelines Initiative, http://www.digitizationguidelines.gov/guidelines/FADGI\%20Federal\%20\%20Agencies\%20Digital\%20Guid elines\%20Initiative-2016\%20Final_rev1.pdf (Accessed April 08, 2018). 
There are different terms associated with the post-processing of images and understanding the differences between them will help clarify which programs are most appropriate to use.

\section{PHOTO-EDITING}

Photo-editing is a catch-all term for the curatorial process of image selection as well as the hands-on manipulation of individual images.

\section{CULLING}

Culling refers to the removal of unwanted images from a larger group. For example, if you are photographing small objects, you will cull the images that are blurry, under or over exposed, or otherwise unwanted. Culling can mean that you either delete the files completely, or simply mark them as rejects in some way.

SUGGESTED PROGRAMS: Lightroom, Bridge, Photo Mechanic

\section{COLOUR-CORRECTING}

Colour-correcting refers to the adjusting of white balance, exposure, contrast, highlights, shadows, colour hue, saturation, etc. of an image.

SUGGESTED PROGRAMS: Lightroom, Capture One, Aperture, Gimp

\section{EDITING}

Editing can refer to either colour-correcting described above, or basic adjustments made to the image, such as de-skewing, cropping, and transforming.

SUGGESTED PROGRAMS: Photoshop, Photoshop Elements, Lightroom, Gimp

\section{RETOUCHING}

Retouching, or more colloquially referred to as 'Photoshopping', is the detailed adjustment and manipulation of elements in a single image, such as compositing, dust removal, colour swapping, etc.

SUGGESTED PROGRAMS: Photoshop, Gimp 


\section{IMPORTING / EXPORTING / SAVE AS}

Importing refers to the ingesting of files into a program so that the user can then manipulate that file. Exporting refers to the 'processing out' or 'saving out' of a file or group of files from any given program as a specific file type. For example, you would import raw files ${ }^{54}$ into Lightroom, edit them in whatever way you need to, then export the files as TIFFs or Jpegs. Saving As and Exporting do similar things, but some programs can only 'Save As' a handful of file types and export as others. If you are working in a program and are unable to save a file as different file type, the function may be available through exporting instead.

54. Raw files, or camera raw files, are digital image files created by a digital camera when a photograph is taken. Raw files contain all of the uncompressed image data generated by the camera when the image is taken, so they are often large files, ranging from $20 \mathrm{mb}$ to $120 \mathrm{mb}$ or more depending on the camera. Naturally, cameras that can produce larger files are more expensive because they have larger image sensors, faster processors, etc. Raw files are often proprietary to the manufacturer of the camera. Some examples include CR2 (Canon), NEF (Nikon), ARW (Sony), DCR (Kodak), RAF (Fuji), SRW (Samsung). DNG is a common camera raw file format that is not proprietary. 


\subsection{Overview of Photoshop Elements}

After you have digitized your materials, you may want to make some adjustments to the file, like cropping or straightening the image, or adjusting the white balance, brightness, and contrast. Adobe Photoshop and Adobe Photoshop Elements are great programs that will allow you to do just that. Photoshop, a word now synonymous with image manipulation, is a versatile program used by photographers, illustrators, and designers. If you are not familiar with Adobe program interfaces, Photoshop may seem overwhelming. It is a powerful program and although I have been using it since middle school, there are some features that I still have yet to use. Thankfully, Adobe offers a simplified version of Photoshop, called Photoshop Elements. Adobe Photoshop Elements, which I will refer to as Elements, is available to try or buy from the Adobe website. Because it is a stripped-down version of the program, it costs significantly less than the full Photoshop program, but has all the tools you will need to do basic post production on your digital image files.

On the left-hand side of the interface, you will see a column of tool icons: the Zoom tool, Hand tool, Quick Selection tool, the Eye tool, Whiten Teeth, Straighten tool, Horizontal Type tool, Spot Healing Brush tool, Crop tool, and Move tool. The tools described here are common in most image-processing programs, so understanding their uses in Photoshop Elements will make it easier to familiarize yourself with other programs.
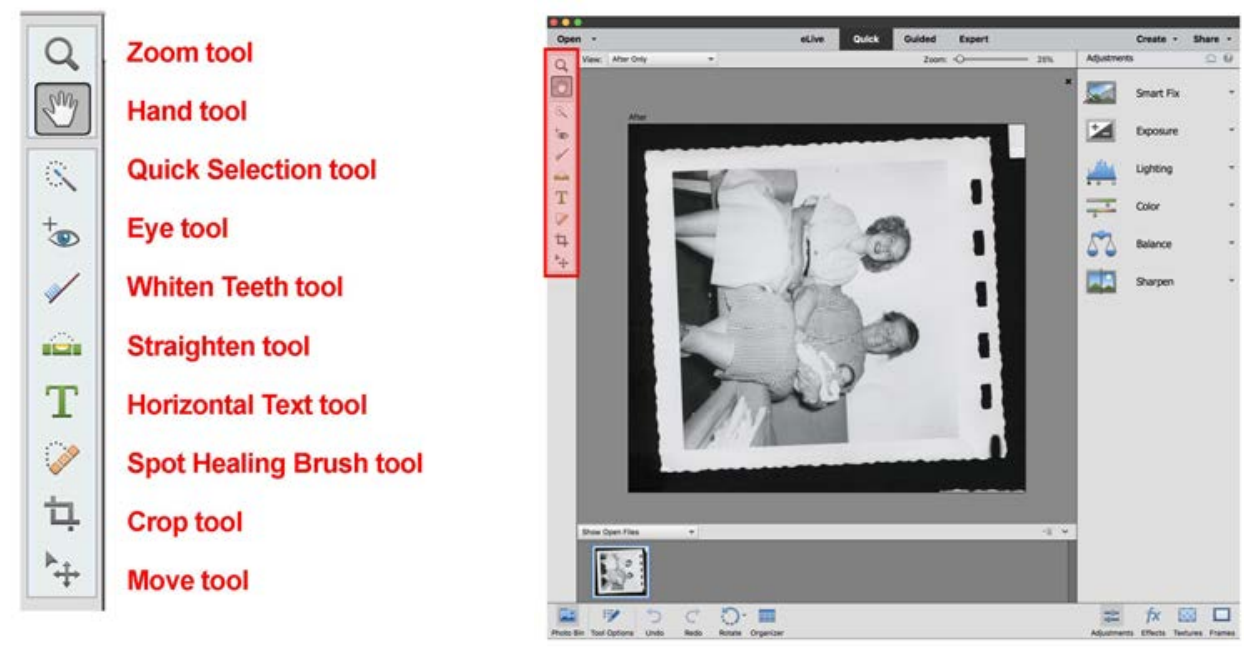


\section{ZOOM TOOL}

The zoom tool allows you to zoom in and out of the open canvas or image, as well as shortcuts to view the image at $100 \%$, to fit the image to the screen, fill the screen, or show the print size of the image.

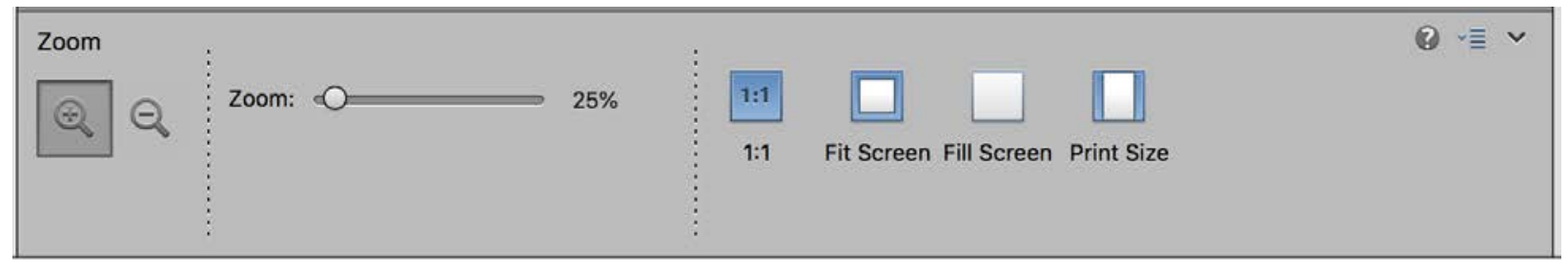

\section{HAND TOOL}

The hand tool allows you to click and hold (grab) the image with the cursor, which is now a small hand icon, and move the image around. This is particularly useful when you are zoomed in on the image and want to shift the visible area of the image without having to zoom out and then zoom back in on a different area.

\section{QUICK SELECTION TOOL}

The quick selection tool allows you to select certain areas of the image, based on pixel value (colour and tone). Although called the "quick" selection tool, it is very powerful and can be used in many different ways. I do not often use this tool when adjusting scans, but it is definitely worth exploring. Do deselect an area you have selected, press Command $+D$ on a Mac or Control+D on a PC.

\section{Quick Selection}

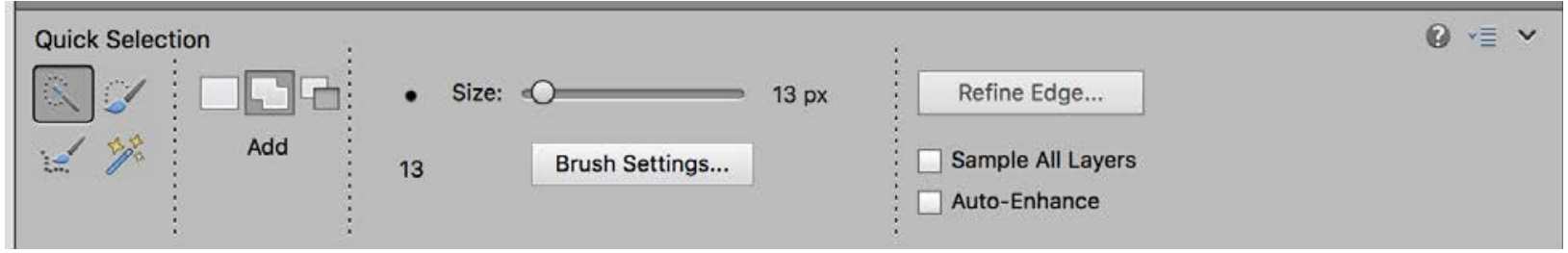




\section{Refine Selection Brush}

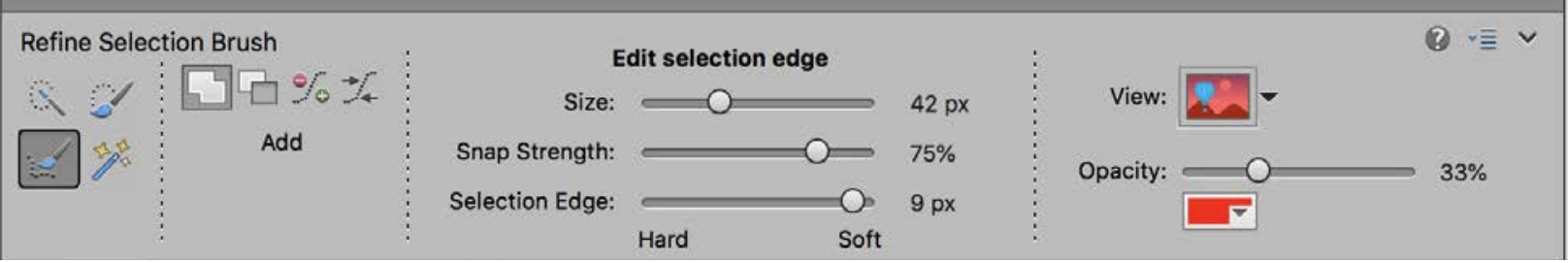

\section{Auto Selection}

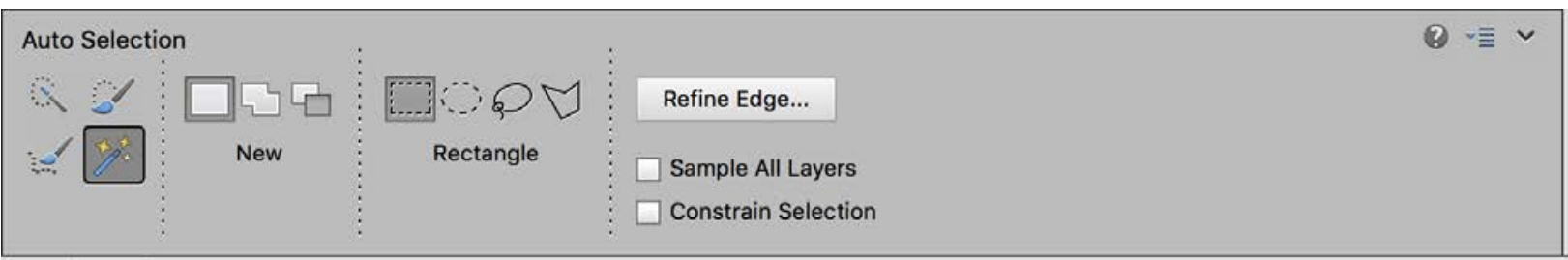

\section{Selection Brush}

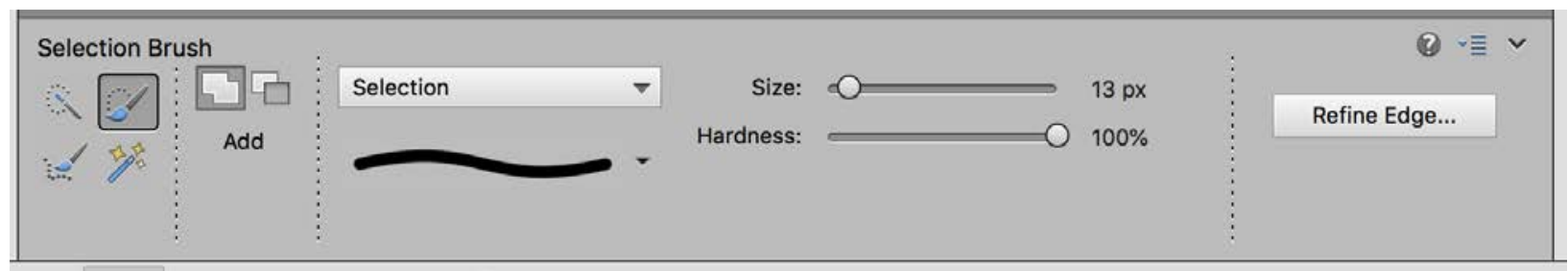

\section{EYE TOOL}

The eye tool allows you to remove red eye from images as well as "correct" closed eyes. I have never used this tool and I will admit from experimenting with it, the results are a bit unsettling! But if you have some group portraits with unsightly red eye and some ill-timed blinking you would like to fix, this tool could be useful.

\begin{tabular}{|c|c|c|c|c|}
\hline Red Eye Removal & & & Closed Eye Correction & (?) \\
\hline Auto Correct & Pupil Radius: $=$ & $50 \%$ & Open Closed Eyes... & \\
\hline$\square$ Pet Eye & Darken: & $50 \%$ & & \\
\hline
\end{tabular}




\section{WHITEN TEETH TOOL}

The whiten teeth tool targets areas (like teeth) that you would like to make whiter. Just used the brush and "paint" over the areas you would like to whiten.

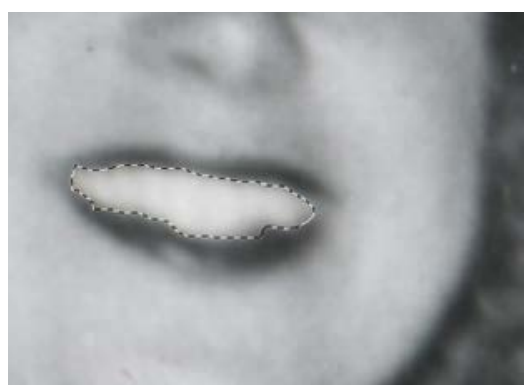

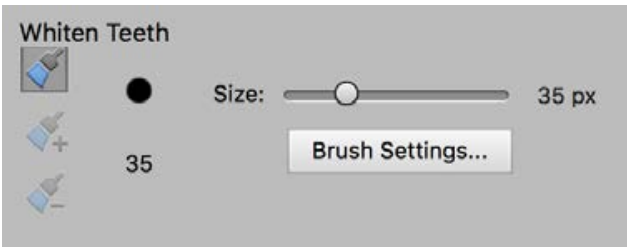

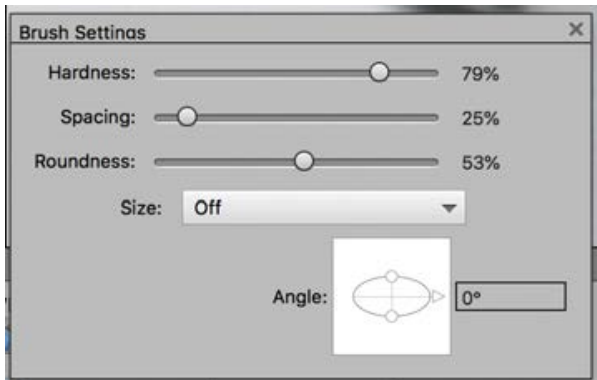

\section{STRAIGHTEN TOOL}

The straighten tool is very useful for correcting slightly skewed images. Sometimes a photograph will move a bit on a flatbed scanner when the lid is closed, and rather than rescan and image multiple times to try to get the image to sit perfectly square, you can straighten the image in post-production.

Straighten

Straighten your photo by drawing a line along an edge that should be horizontal or vertical.
Select an option to crop/resize the image and how to fill the empty spaces.

Maintain Image Size

$\checkmark$ Autofill edges

Checking the box next to Autofill edges will automatically fill the new areas of the canvas with the colour of the border areas, in this case black.
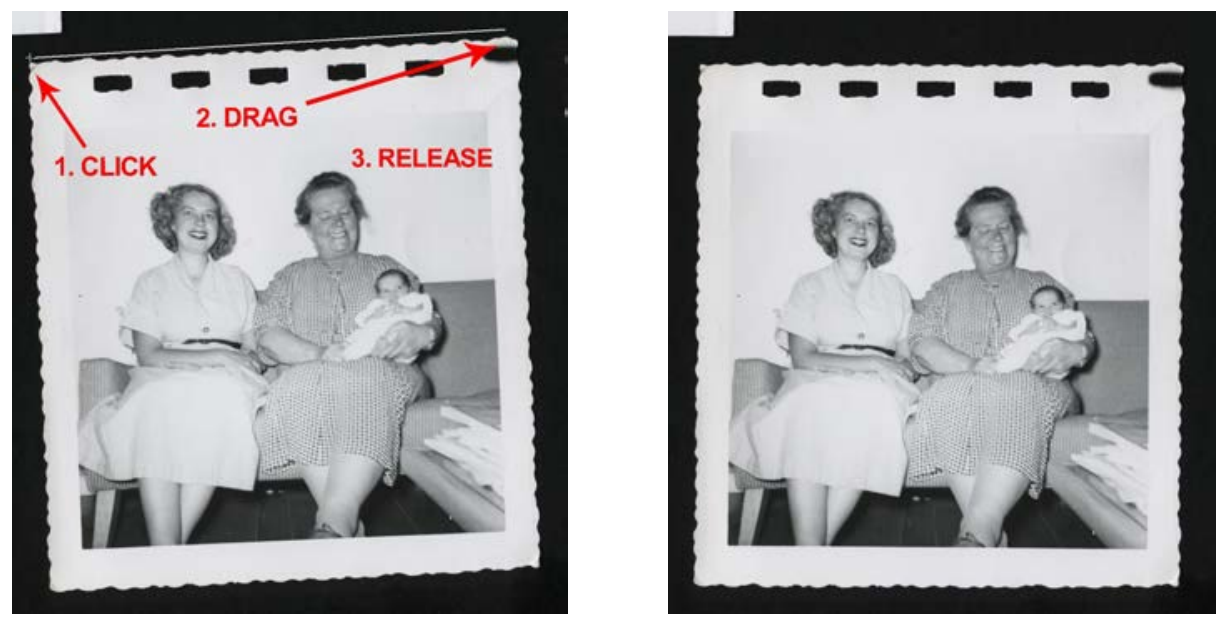


\section{HORIZONTAL TEXT TOOL}

The horizontal text tool allows you to write text over a canvas or image. You can also write vertical text, write text on a shape, create a selection in the shape of text, type text along a specific shape, and more.

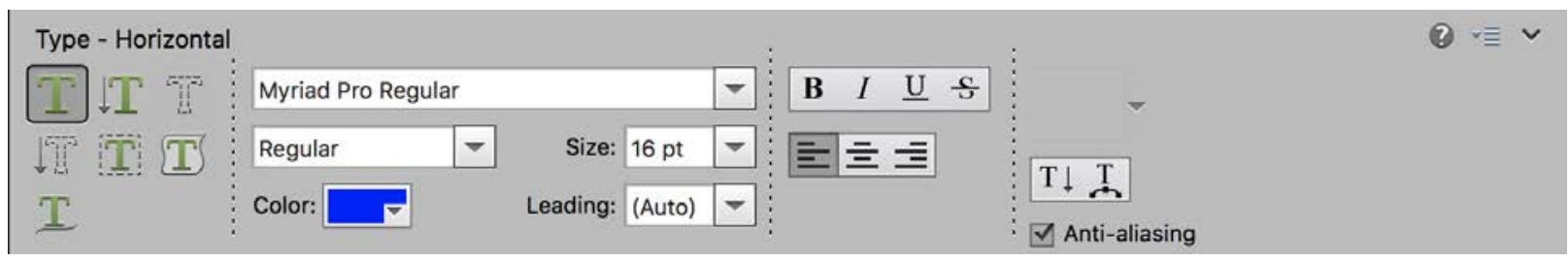

\section{SPOT HEALING BRUSH TOOL}

The spot healing tool, represented by a band aid icon, is used to "heal" areas of images, like dust, tears, or other small blemishes. It does so by sampling the pixels around the brush area and then filling in the selection area with similar pixels. This tool is especially useful for retouching out dust particles from scans of slides, negatives, and prints.

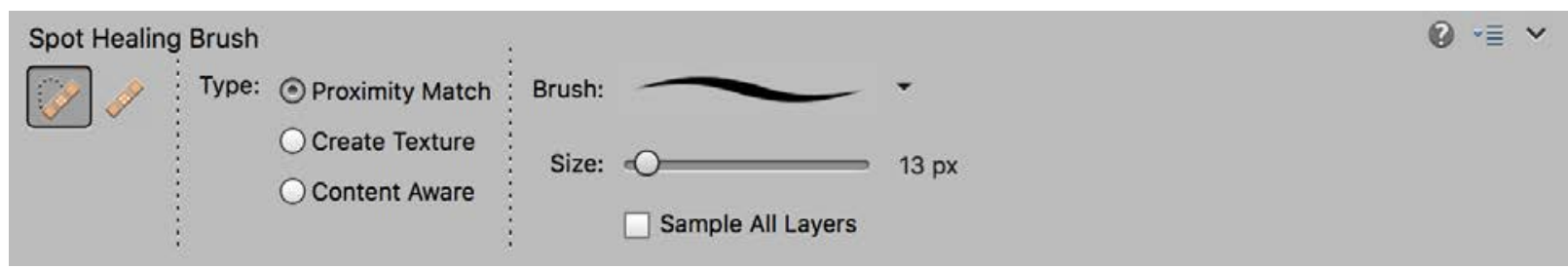

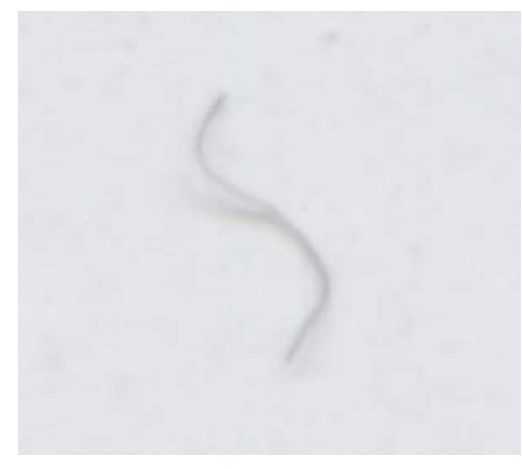

Small fiber on scan

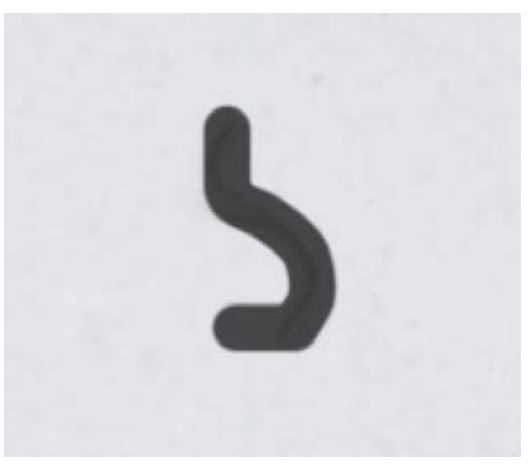

Healing brush drawn over

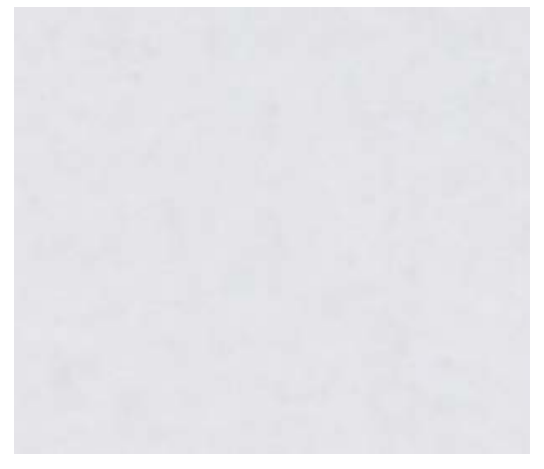

Pixels filled in 


\section{CROP TOOL}

The crop tool lets you trim off excess areas around the perimeter of the image, or crop the canvas to a particular area of the image. You can designate the width and height you want the cropping ratio to be, as well as the resolution. To crop an image, simply click and drag across the image. You can also rotate an image by drawing a crop selection then clicking, holding and dragging along one of the eight perimeter anchors of the frame.

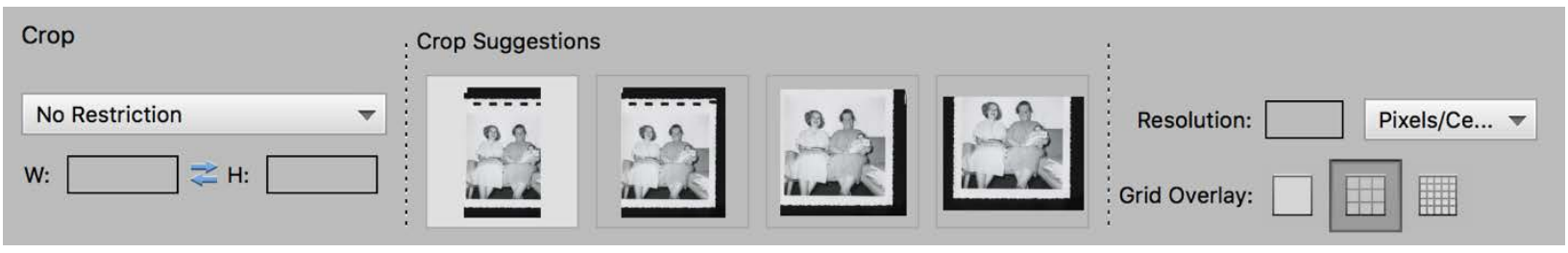

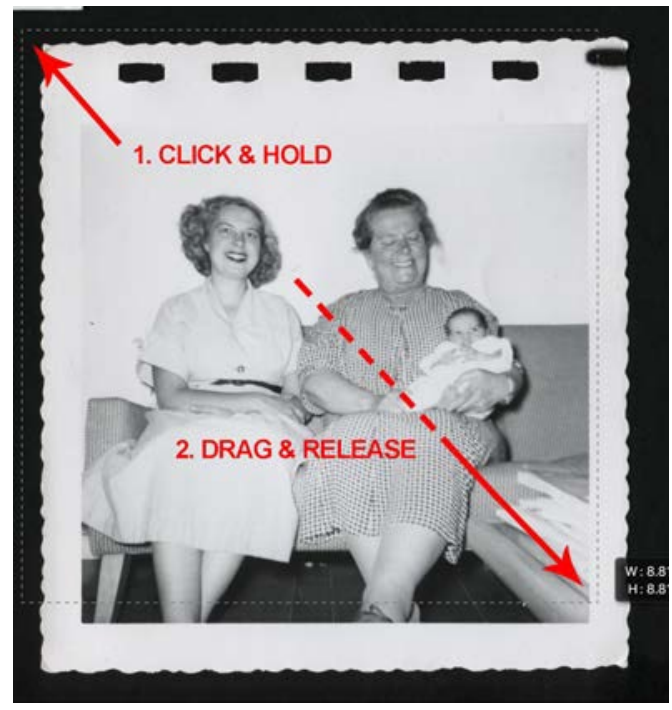

2. Once you release the cursor, you will see cropping window appear over the area you have selected. The area outside of the cropping area, the part of the canvas that will be discarded after you finalize the crop, appears with a semitransparent mask overlay.

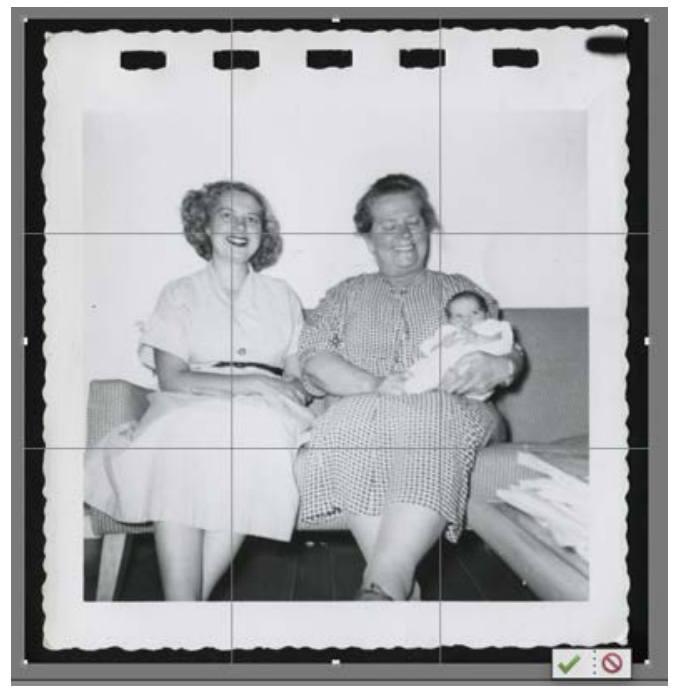


3. You can adjust the cropping frame by clicking and dragging one of the either anchor points around the perimeter of the frame. You can also rotate the cropping frame by hovering near one of the anchors with the cursor until a curved arrow appears, and then click and drag. You will see the cropping frame skew to reflect the amount of rotation you have created by dragging the cursor.

4. To finalize the crop, press Enter or click the green check mark in the lower right corner.

5. If you have drawn a crop and decide you want to start again, simple press Escape or click the red circle icon in the lower right corner.
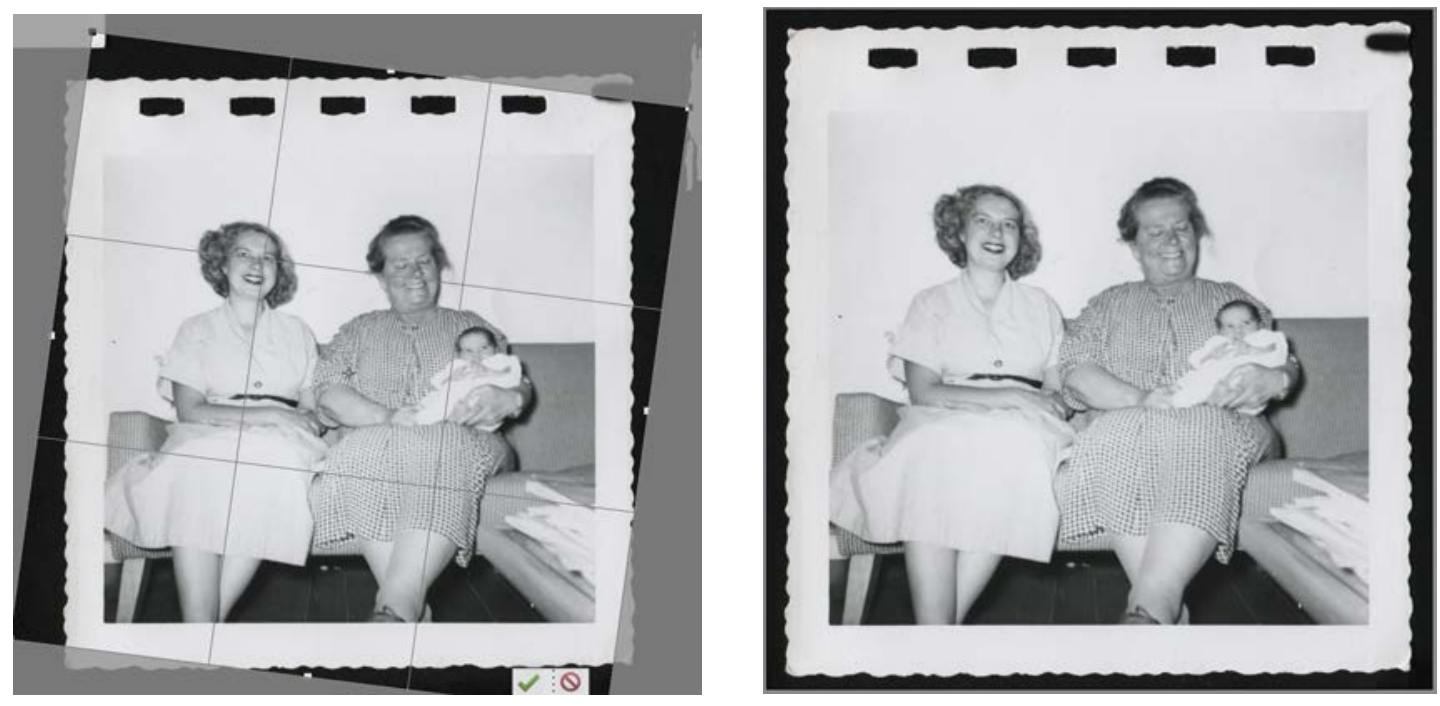

\section{MOVE TOOL}

The move tool allows you to select an object or layer in Elements and move it around the canvas. You can change the order of objects so that some are in front of or on top of others, as well as align objects along a different axis.

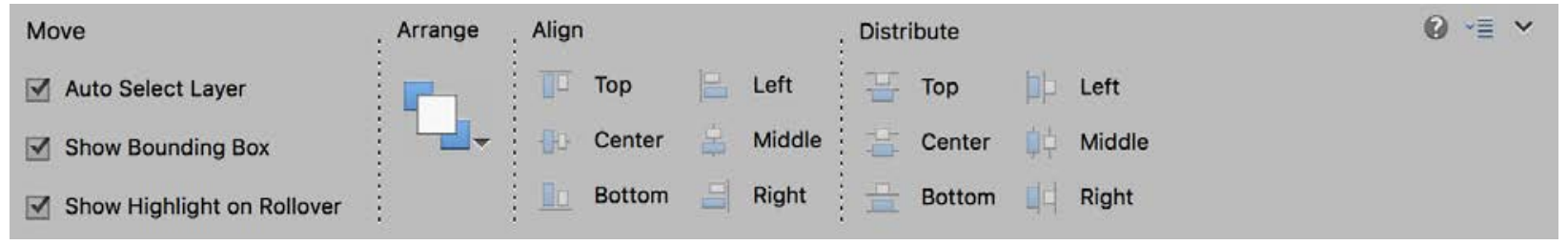




\section{OPTIONS MENU}

At the very bottom of the Elements window is a horizontal toolbar.

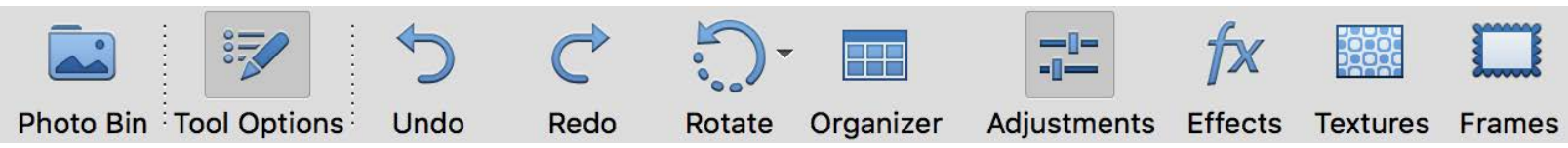

PHOTO BIN shows or hides the Photo Bin pane described above.

TOOL OPTIONS shows or hides the options pane for each tool described above.

UNDO and REDO allow you to undo or redo adjustments you have made to the image.

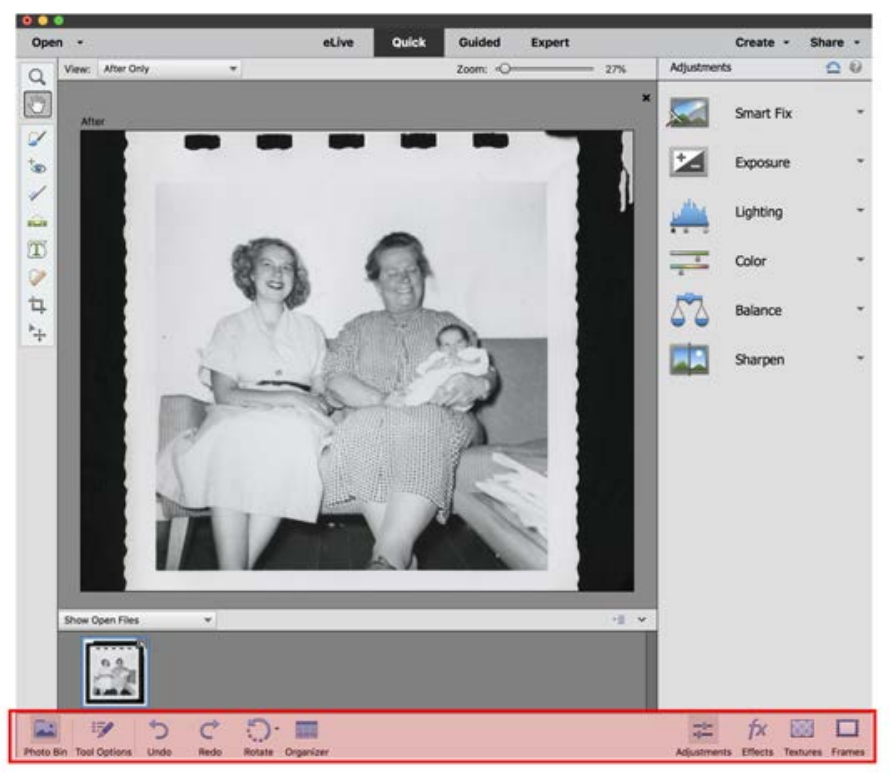

ROTATE allows you to rotate the image. Each click of the rotate button turns the canvas $90^{\circ}$ counterclockwise or clockwise (available by clicking on the small drop down arrow next to the rotate icon).

The ORGANIZER button launches the Elements Organizer program, which is used to search and organize all of the images on your computer or external hard drive based on people, places, and events.

The ADJUSTMENTS icon shows or hides the adjustments toolbar on the right side of the interface. 
The EFFECTS button allows you to apply pre-programmed develop settings to your image to mimic various analog film styles, much like the filters available on various social media platforms.

The TEXTURE button allows you to apply various semi-transparent textures to your image to achieve different visual effects or styles.

Similarly, the FRAMES button allows you to add any number of pre-programmed frames to your image.

\section{ADJUSTMENTS MENU}

The adjustments menu contains tools to adjust the brightness, contrast, color balance, tint, and sharpness of your image. These tools are intended to be used with photographs or photographic images. The best way to understand what these tools can do is to click through them and see the adjustments they make.

\section{SMART FIX}

The smart fix adjustment panel offers nine automatic combinations of exposure, brightness, and contrast that you can quickly apply to your image.

\section{EXPOSURE}

The exposure panel offers nine automatic exposure adjustments to either increase (brighten) or decrease (darken) the exposure.

\section{LIGHTING}

The lighting panel allows you to increase the brightness of the shadows, midtones, and highlights individually. 


\section{COLOUR}

The colour adjustment panel allows you to increase or decrease the color saturation, hue, or vibrance of your image.

\section{BALANCE}

The balance adjustment panel allows you to increase or decrease the temperature of the image (make it warmer or cooler) as well as the tint (green or magenta).

\section{SHARPEN}

The sharpen adjustment panel allows you to increase the sharpness of the image (the contrast around edges in the image). This can help make fuzzy images look slightly sharper.

\section{GUIDED and EXPERT TAB}

The guided and expert tabs are other ways to use the tools Elements depending on one's familiarity with the program. The guided tab illustrates different adjustments you can make to your image with interactive sliders to see images before and after each respective change. The Expert tab is similar to that of Photoshop, allowing you to make finer adjustments to your images beyond what is suggested in the Quick tab. 


\subsection{Editing Images In Photoshop Elements}

1. Open the Adobe Photoshop Elements program.

2. Once the program has opened, click the OPEN button in the upper left corner of the interface.
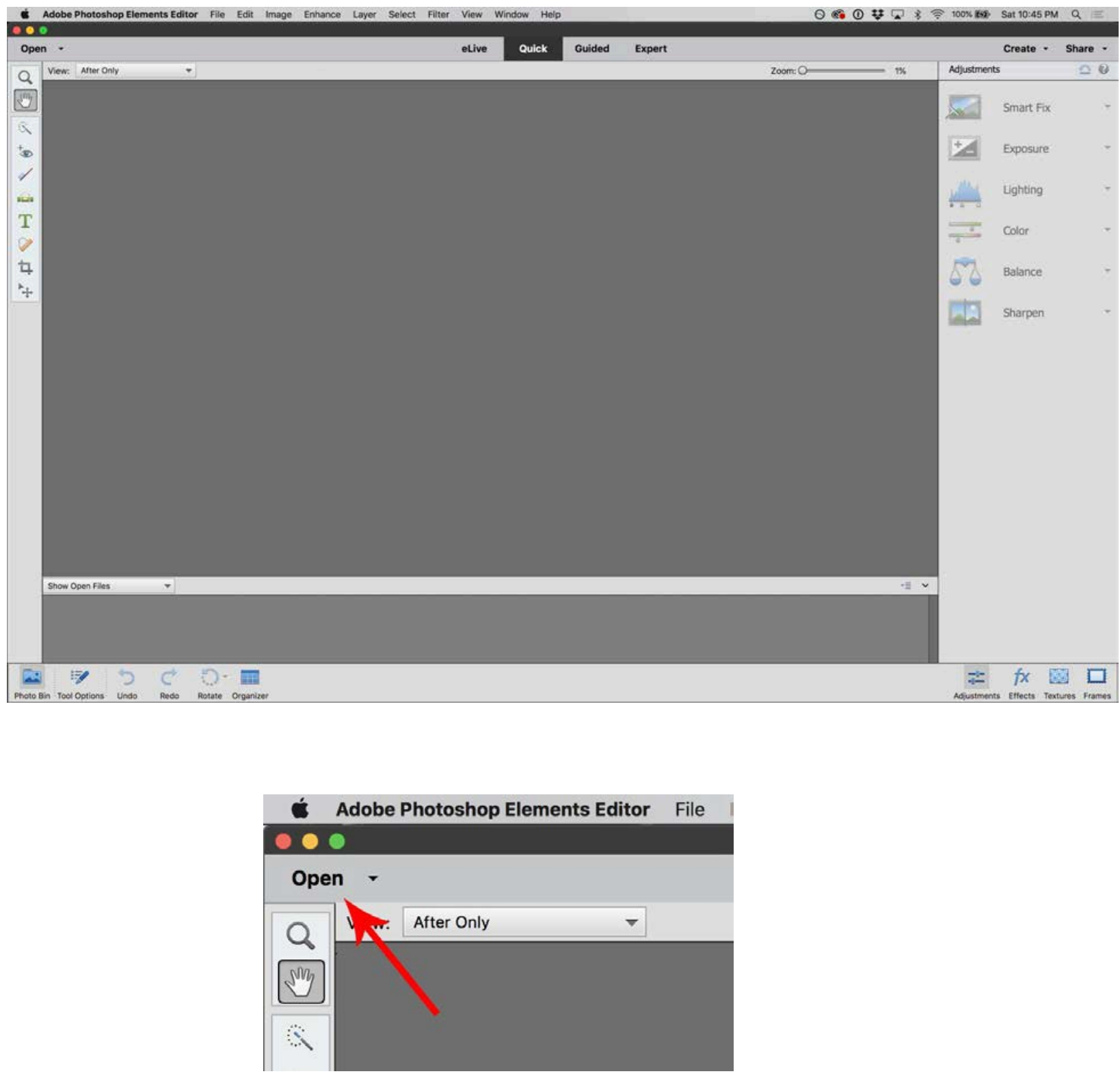
3. Select the file you want to work on and click OPEN.

\begin{tabular}{|c|c|c|c|c|c|}
\hline 国 & $\because \equiv$ & Master & Tiffs & & Q Search \\
\hline \multicolumn{2}{|l|}{ Name } & ^ & Date Modified & Size & Kind \\
\hline \multicolumn{2}{|r|}{ KAUFMAN_022.tif } & & Mar 31, 2018 at 10:16 PM & $39.2 \mathrm{MB}$ & TIFF image \\
\hline \multicolumn{2}{|c|}{ EAUFMAN_023.tif } & & Mar 31, 2018 at 10:19 PM & $69.2 \mathrm{MB}$ & TIFF image \\
\hline \multicolumn{2}{|c|}{ Ex KAUFMAN_024.tif } & & Mar 31,2018 at $10: 23$ PM & $69.6 \mathrm{MB}$ & TIFF image \\
\hline \multicolumn{2}{|c|}{ n KAUFMAN_025.tif } & & Mar 31,2018 at $10: 26$ PM & $69.2 \mathrm{MB}$ & TIFF image \\
\hline \multicolumn{2}{|c|}{ 프 KAUFMAN_026.tif } & & Mar 31,2018 at $10: 27$ PM & $69.6 \mathrm{MB}$ & TIFF image \\
\hline \multicolumn{2}{|c|}{ 圆 KAUFMAN_027.tif } & & Mar 31,2018 at $10: 28$ PM & $79.1 \mathrm{MB}$ & TIFF image \\
\hline \multicolumn{2}{|c|}{ 圖 KAUFMAN_028.tif } & & Apr 1,2018 at $11: 22$ AM & 74.1 MB & TIFF image \\
\hline \multicolumn{2}{|c|}{ [ KAUFMAN_029.tif } & & Apr 1, 2018 at $11: 23$ AM & $75.9 \mathrm{MB}$ & TIFF image \\
\hline \multicolumn{2}{|c|}{ III KAUFMAN_030.tif } & & Apr 1,2018 at $11: 25$ AM & $74.8 \mathrm{MB}$ & TIFF image \\
\hline \multicolumn{2}{|c|}{ 国 KAUFMAN_031.tif } & & Apr 1,2018 at $11: 30$ AM & $73 \mathrm{MB}$ & TIFF image \\
\hline \multicolumn{2}{|c|}{ 圆 KAUFMAN_032.tif } & & Apr 1,2018 at $11: 31$ AM & $76 \mathrm{MB}$ & TIFF image \\
\hline \multicolumn{2}{|c|}{ 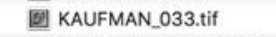 } & & Apr 1, 2018 at $11: 32$ AM & $74.9 \mathrm{MB}$ & TIFF image \\
\hline \multicolumn{2}{|c|}{ 国 KAUFMAN_090-001.tif } & & Apr 1,2018 at 5:54 PM & $50 \mathrm{MB}$ & TIFF image \\
\hline \multicolumn{2}{|c|}{ 므 KAUFMAN_090-002.tif } & & Apr 1, 2018 at 5:57 PM & $50 \mathrm{MB}$ & TIFF image \\
\hline \multicolumn{2}{|c|}{ 国 KAUFMAN_091.tif } & & Apr 2, 2018 at 7:04 PM & $57.3 \mathrm{MB}$ & TIFF image \\
\hline \multicolumn{2}{|r|}{ 区 KAUFMAN_092-001.tif } & & Apr 2, 2018 at 7:07 PM & $78.3 \mathrm{MB}$ & TIFF image \\
\hline \multicolumn{2}{|r|}{ ] KAUFMAN_092-002.tif } & & Apr 2, 2018 at 7:09 PM & $92.6 \mathrm{MB}$ & TIFF image \\
\hline \multicolumn{2}{|r|}{ GAUFMAN_093-001.tif } & & Apr 2, 2018 at 7:11 PM & $92.6 \mathrm{MB}$ & TIFF image \\
\hline \multicolumn{2}{|c|}{ GAIIFMANn3 } & & Anr 1 2M18 at 12:11 PM & 9) Q MR & TIFF imane \\
\hline \multicolumn{2}{|c|}{ Options } & & & Cancel & Open \\
\hline
\end{tabular}

4. The file will now open in the QUICK tab.

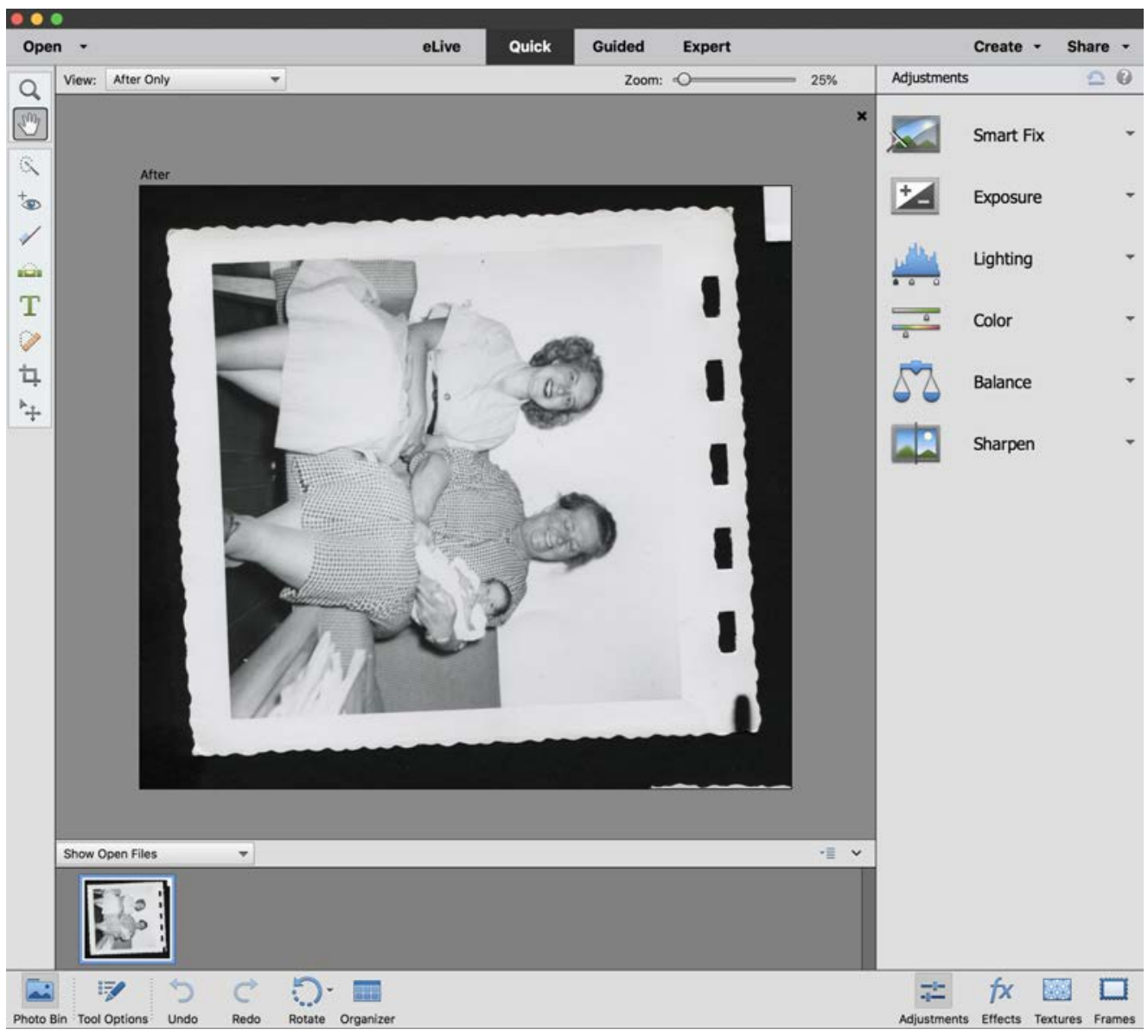


5. Click the ROTATE button at the bottom of the screen as necessary to orient the image correctly.

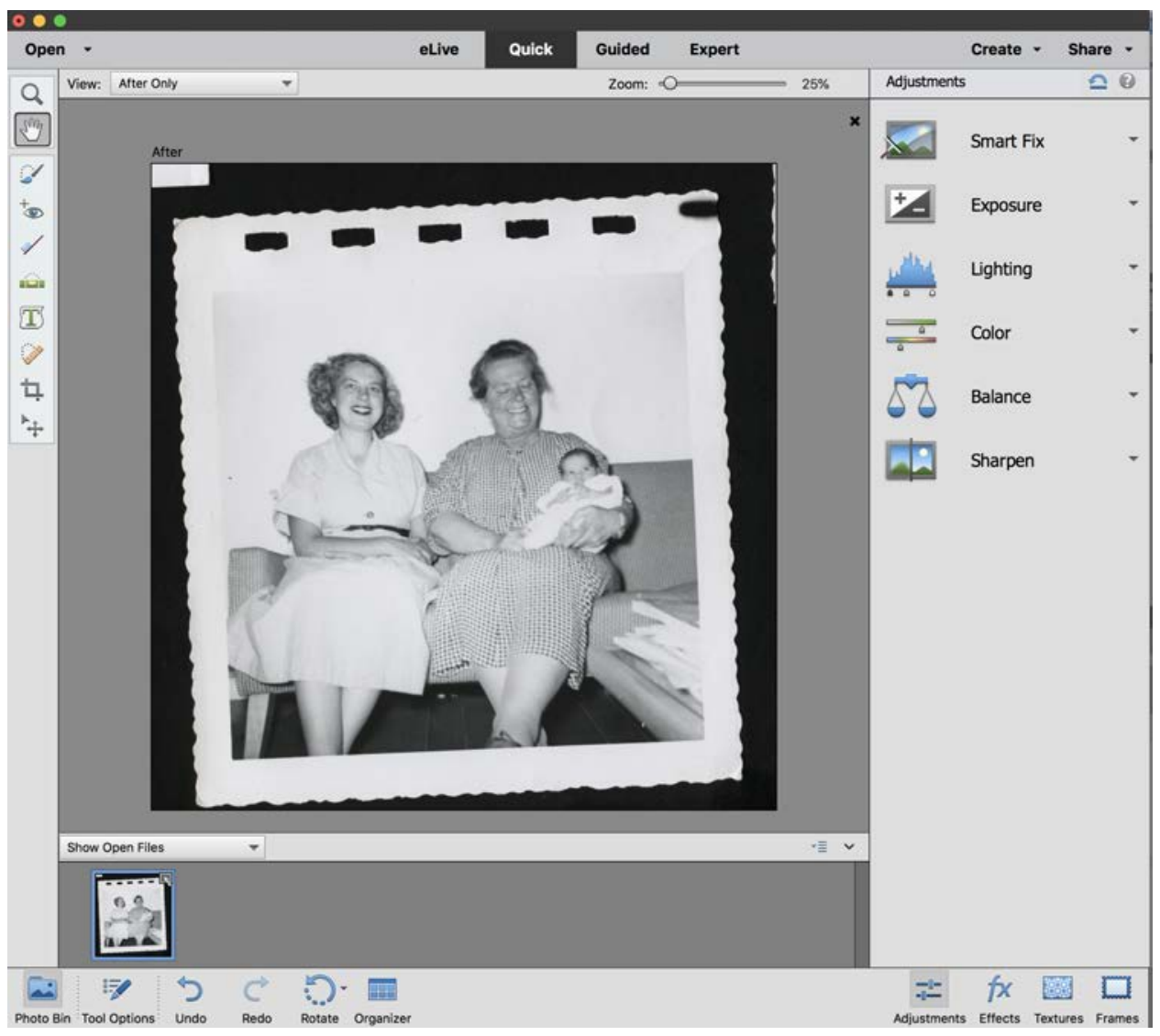

6. Then use the Straighten tool located in the left vertical toolbar to draw a line along an edge that you would like to be made perfectly horizontal, such as the outer edge of the image. Leave the box next to Autofill Edges checked if you would like the program to automatically fill the new areas on the canvas created by the rotation to be filled in with the automatically detected background colour. If you leave this box unchecked, the new canvas spaces will automatically appear white, as seen below. 

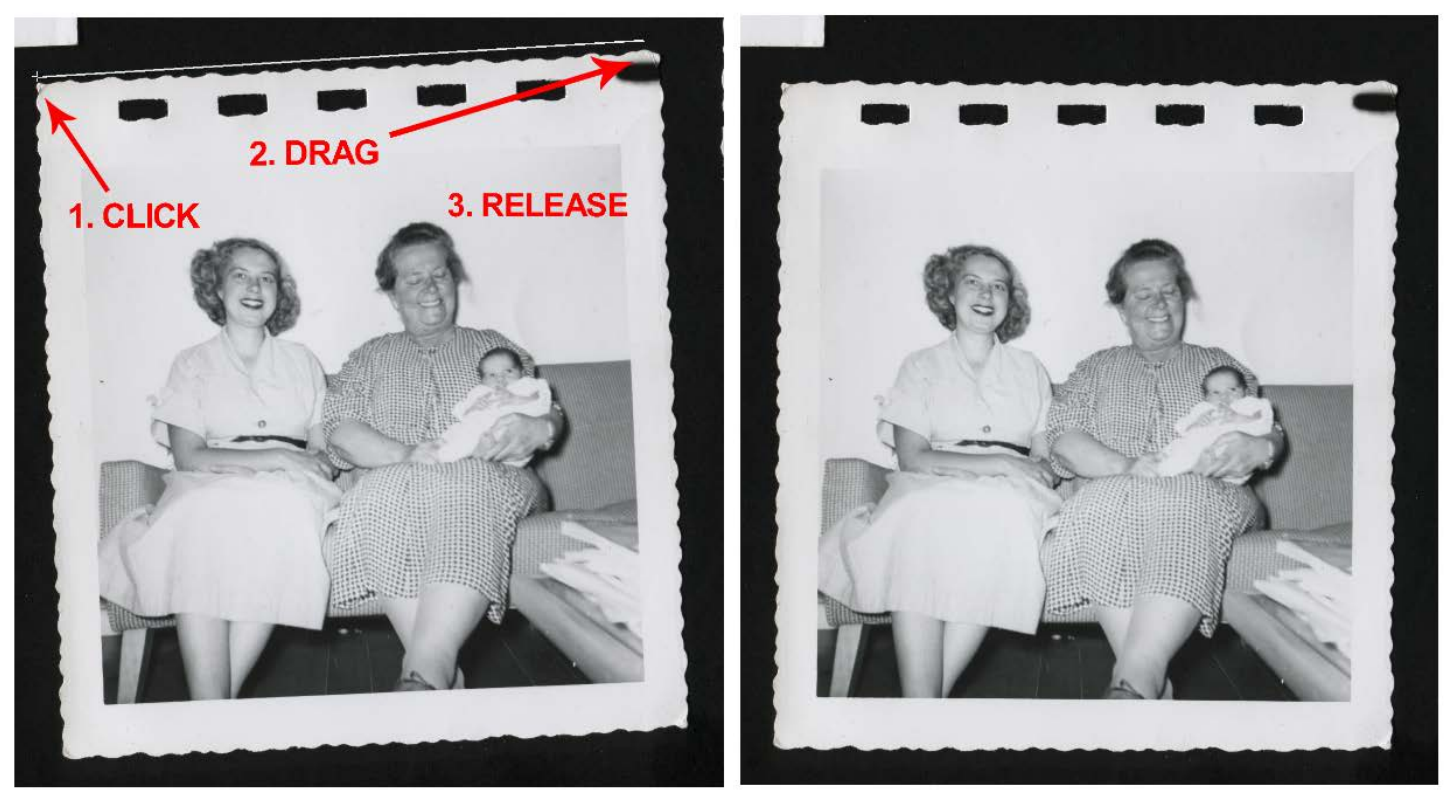

Straighten

Straighten your photo by drawing a line along an edge that should be horizontal or vertical.

Select an option to crop/resize the image and how to fill the empty spaces.

Maintain Image Size

$\checkmark$ Autofill edges

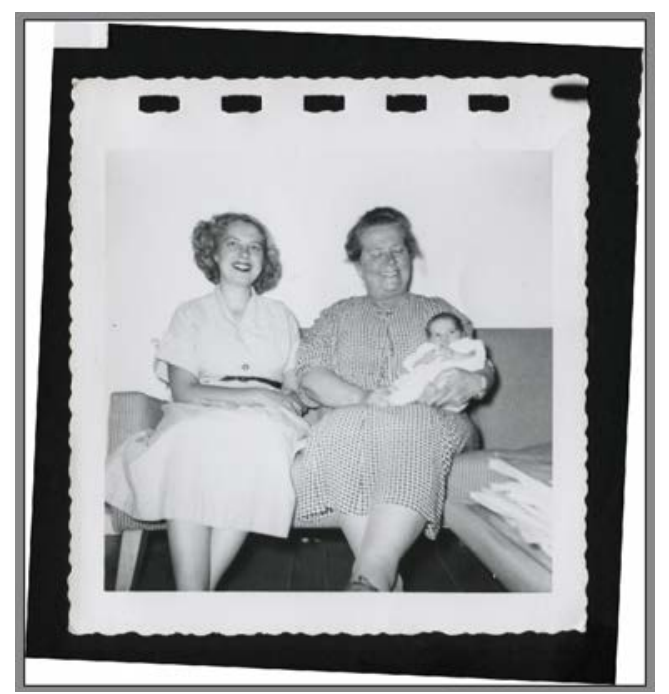

Background canvas space automatically filled in with white.

7. Now that the image has been straightened, you may want to crop in more tightly around the image. Do do this, select the CROP tool in the left vertical toolbar. If a cropping frame automatically appears, drag the corners to the desired location. If 
no cropping frame appears automatically, use your cursor to draw a frame around the image. The frame can be easily adjusted once it has been draw. Once the cropping frame is positioned satisfactorily, press Enter or Return to apply the crop.
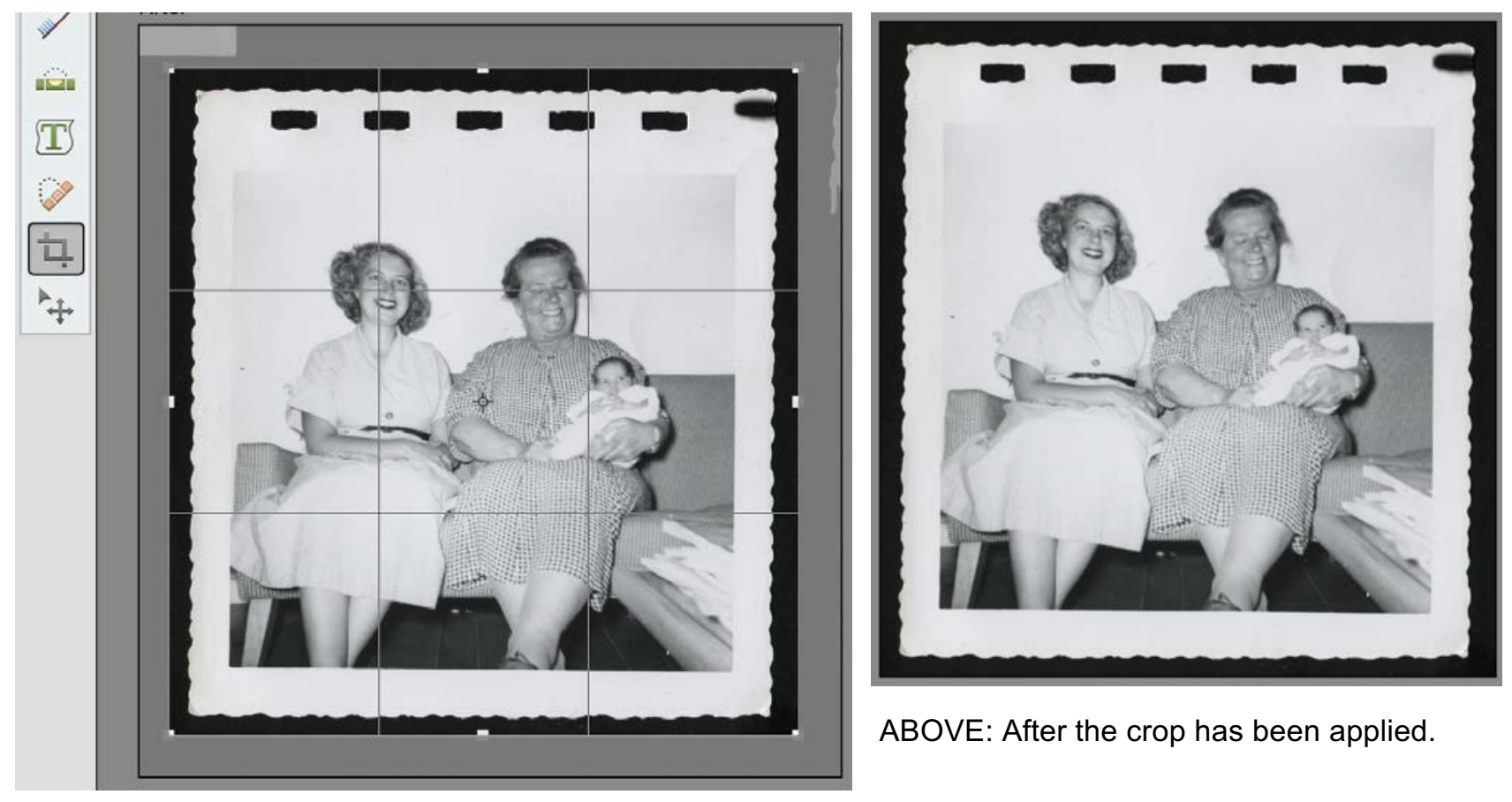

ABOVE: After the crop has been applied.

If you want to UNDO any adjustment, simply click EDIT in the top menu bar, and click Undo. You can also press Control and Z on a PC keyboard, or Command and $Z$ on a Mac.

8. To save your image, click FILE then SAVE.

9. When the Save dialogue box appears, locate the folder where you would like to save the file, make sure that the selected format is set to TIFF, and then click SAVE. 


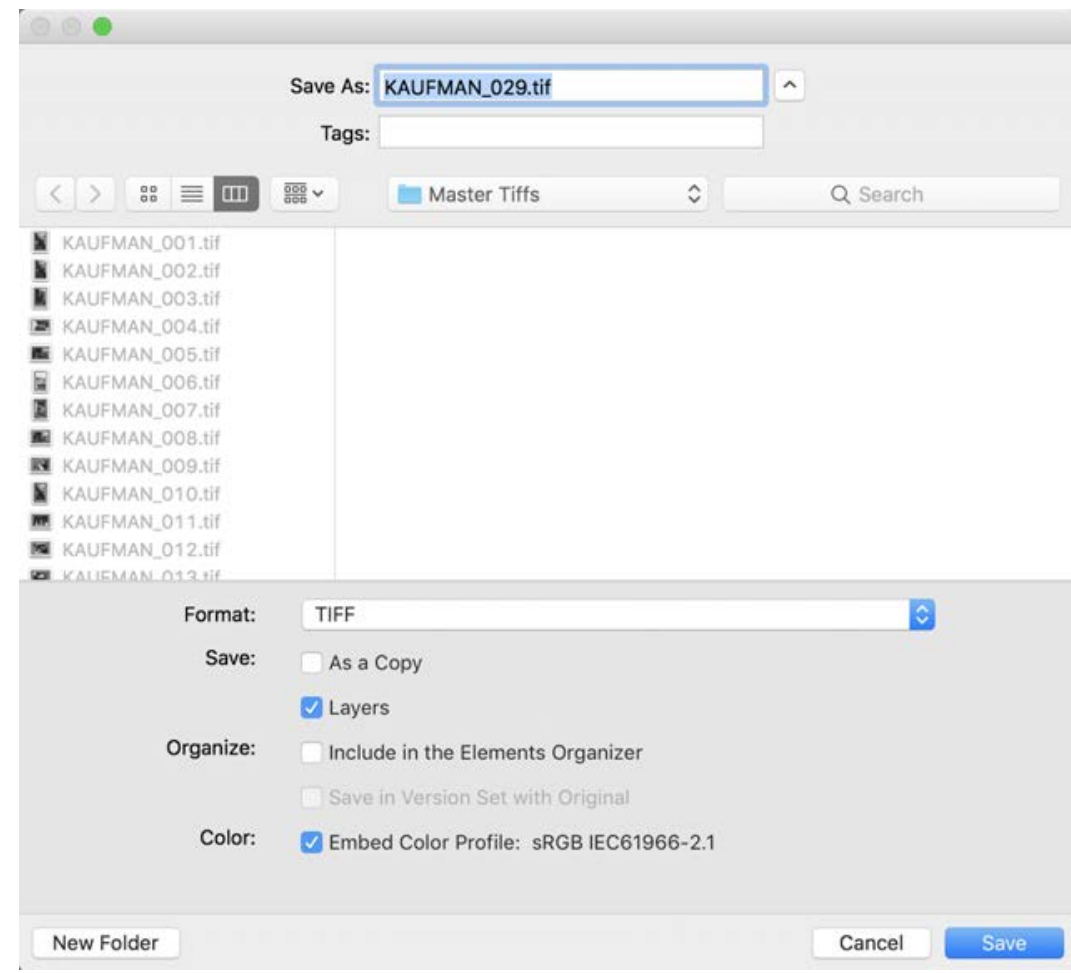

10. If you see a warning that a file with that name already exists, click REPLACE. It is ok to overwrite the original file in this instance because the only adjustments you have made to the image are the rotation and the crop.

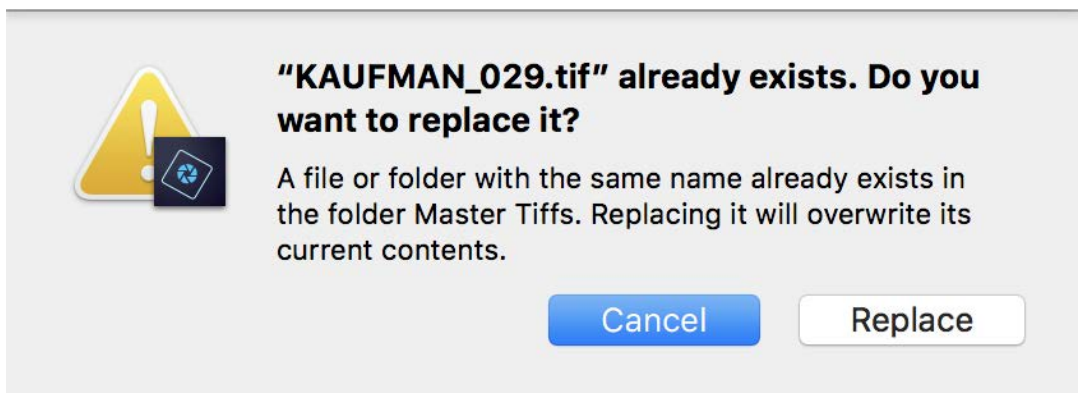

11. Next, the TIFF Options box will appear. Check that the following options are selected:

a. Image Compression: 'LZW'

b. Pixel Order: 'Interleaved RRGGBB'

c. Byte Order: [Whichever option your computer selects as the default.] 
d. Layer Compression: 'RLE (faster saves, bigger files)'

\begin{tabular}{|c|c|c|c|}
\hline \multicolumn{4}{|c|}{ TIFF Options } \\
\hline & & OK \\
\hline \multirow{2}{*}{\multicolumn{2}{|c|}{$\begin{array}{l}\text { ONone } \\
\text { ○ LzW }\end{array}$}} & () Interleaved (RGBRGB) & \\
\hline & & Per Channel (RRGGBB) & Cancel \\
\hline & & \\
\hline \multicolumn{2}{|l|}{ O JPEG } & OIBM PC & \\
\hline Quality: & Maximum $\boldsymbol{\nabla}$ & ( ) Macintosh & \\
\hline small file & large file & $\begin{array}{l}\text { Layer Compression } \\
\text { RLE (faster saves, bigger files) }\end{array}$ & \\
\hline$\square$ Save Imac & nid & ZIP (slower saves, smaller files) & \\
\hline & & Discard Layers and Save a Copy & \\
\hline
\end{tabular}

12. Click OK.

13. If a message appears saying that 'Including layers may increase file size', click OK.

Adobe Photoshop Elements

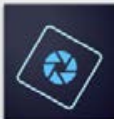

Including layers will increase file size.

Don't show again

Cancel

OK

14. Your image has now been saved and you can close the file by clicking the small ' $X$ ' in the top right side of the image, or by clicking File and Close. You can also press Command $+\mathbf{W}$ if working on a Mac computer or Control $+\mathbf{W}$ on a PC. 


\subsection{Editing Images In Photoshop Lightroom}

1. Open the Adobe Photoshop Lightroom

Program.

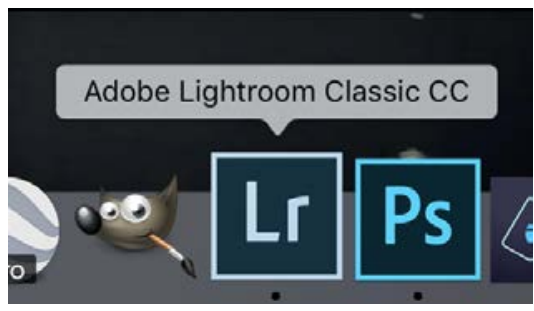

2. If Lightroom can't find a catalog, click Choose a Different Catalog.

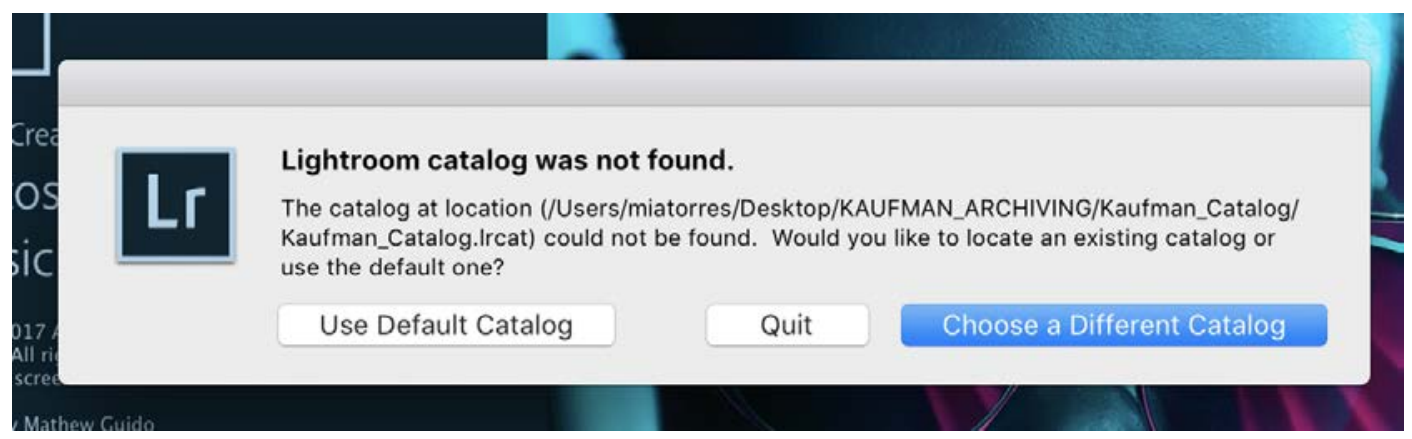

3. Click Create a New Catalog.

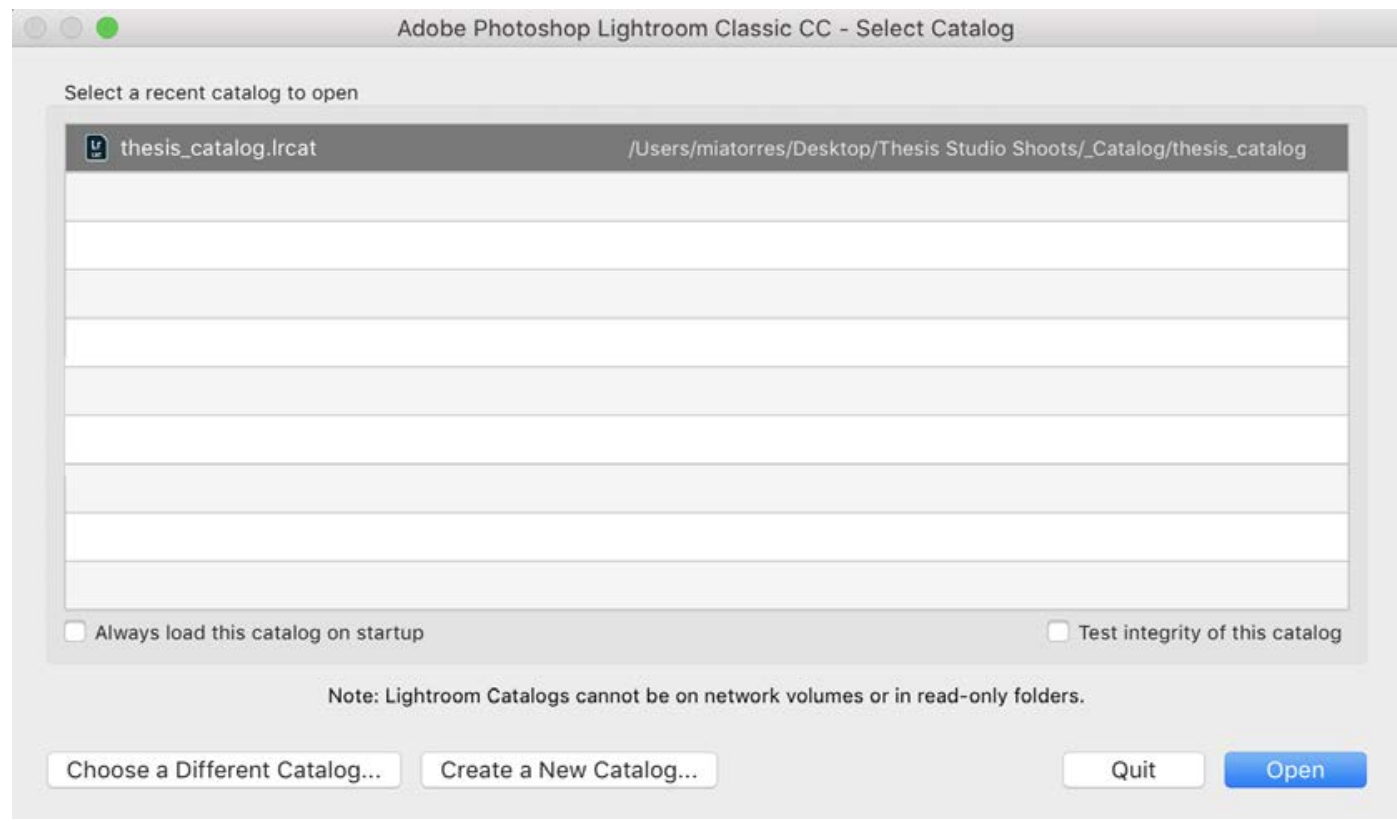


4. Name the catalogue you would like to make, then click CREATE. Note that Lightroom will create a folder with this name and place the catalog file with the same name in that folder.

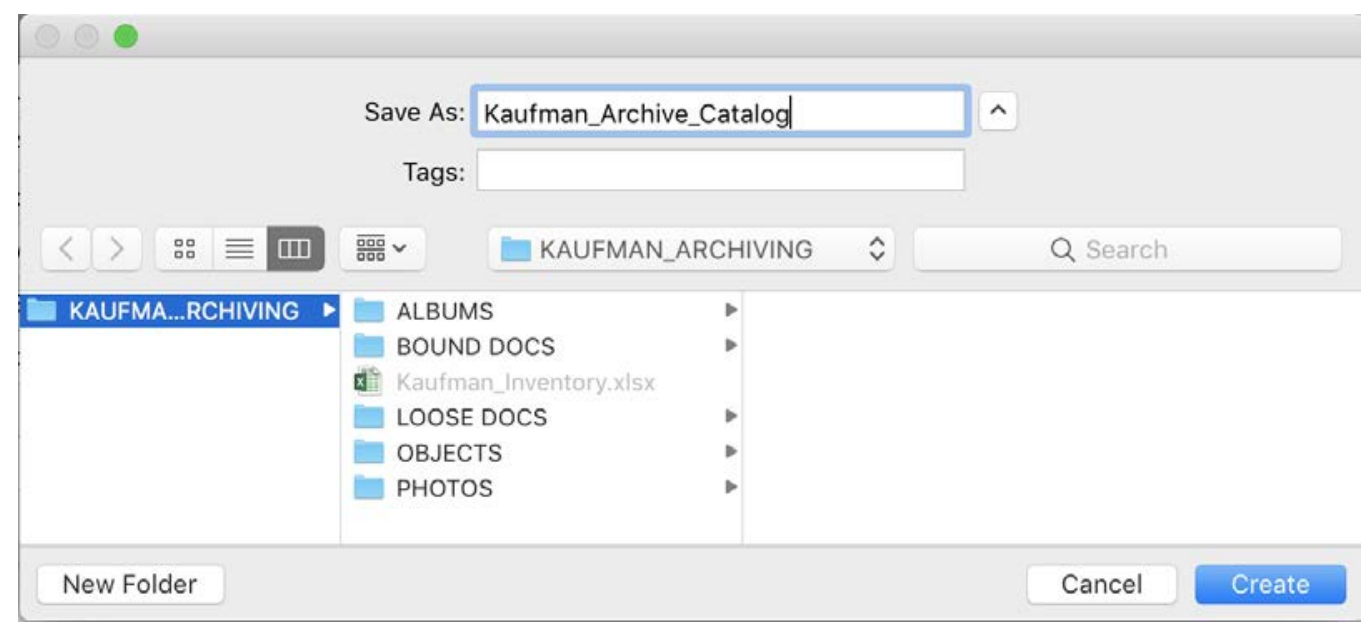

5. The Lightroom interface will now open.

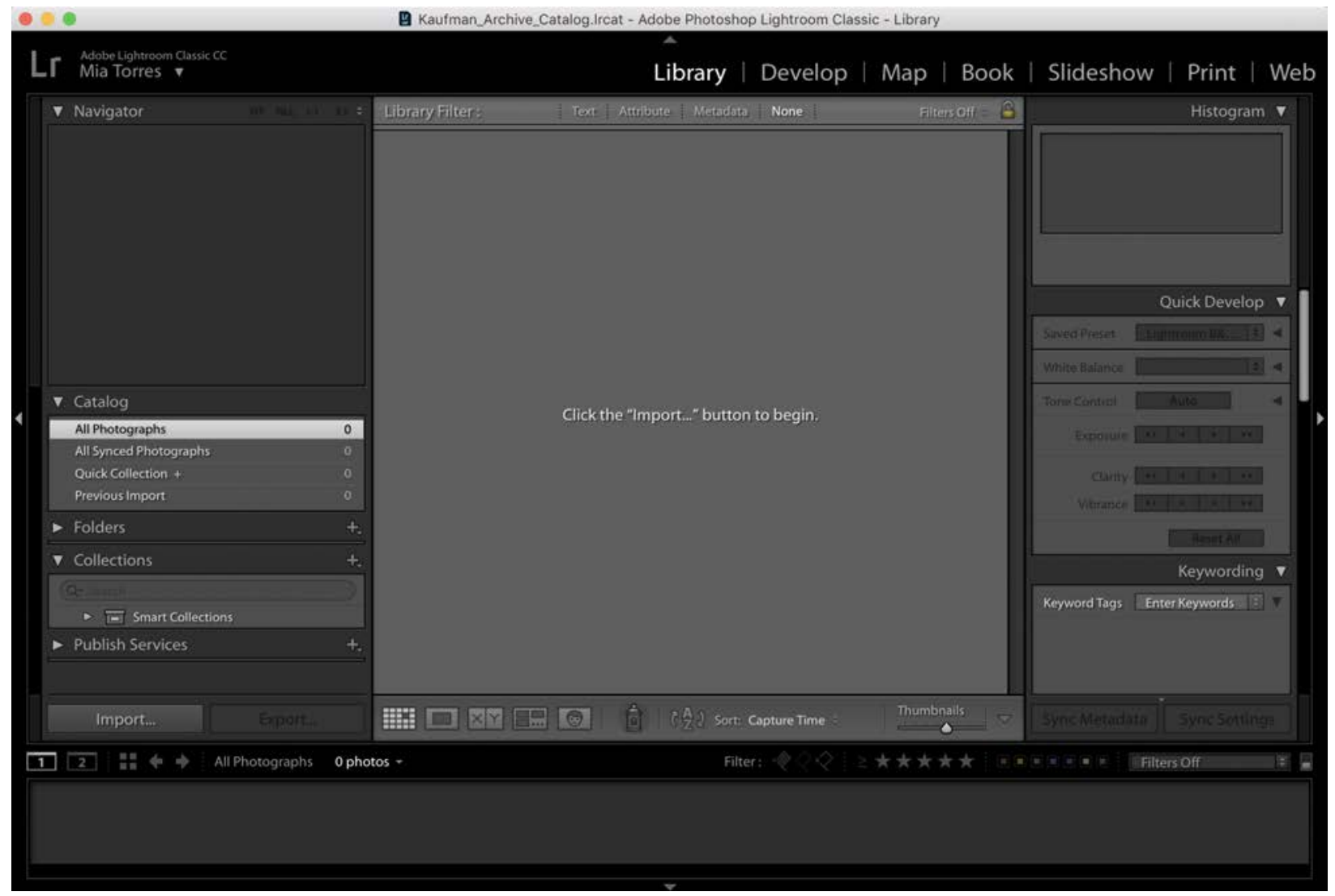


6. To import images from your computer, click IMPORT.

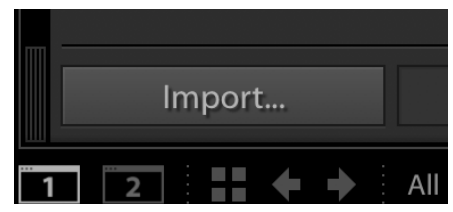

7. The Import interface will now open. In the Source panel on the left side, navigate to the location of the files you want to import.
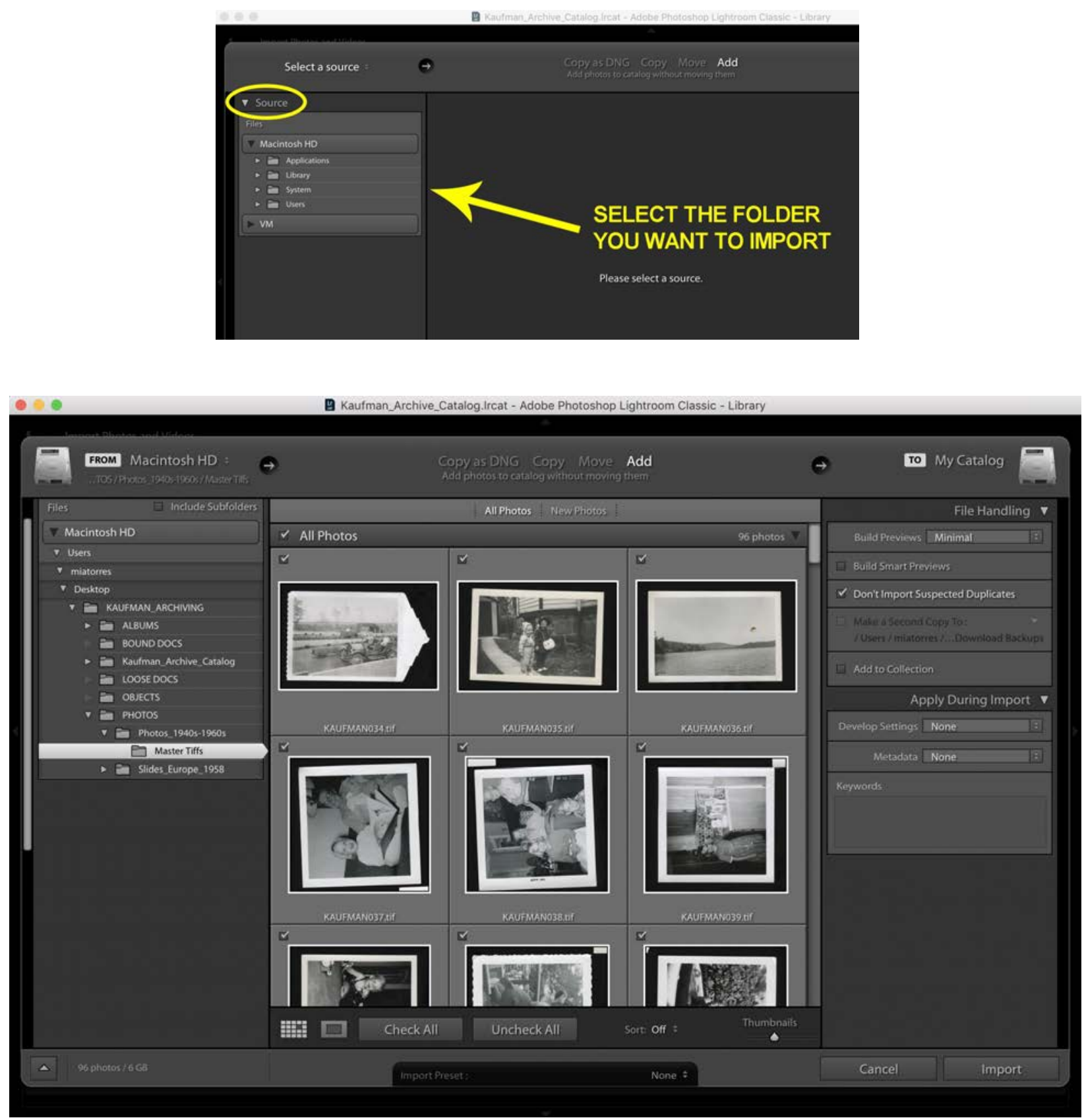


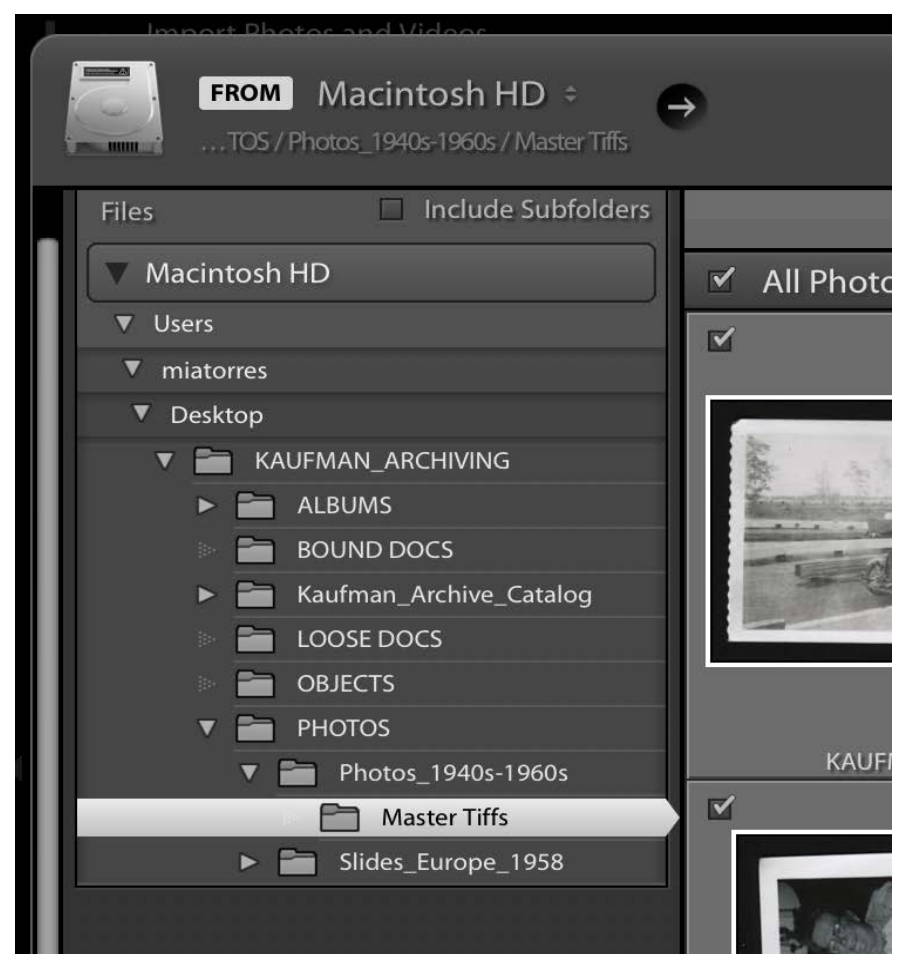

8. Now you can either select all the images or only the ones you want. You can sort the images by capture time (the time at which the original file was created), checked state (whether or not you have selected the image), file name, file type, or media type. Then click IMPORT.

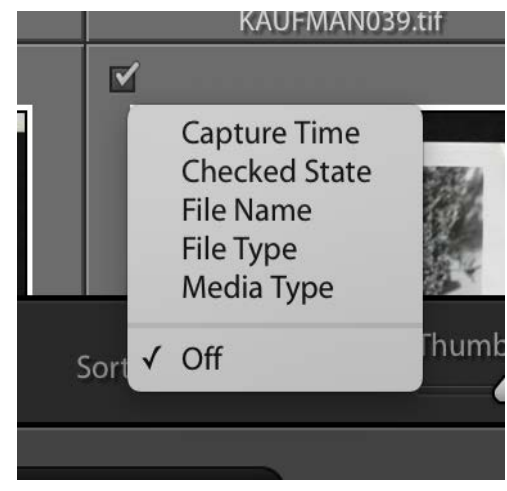

9. Lightroom will now import the files into the catalog.

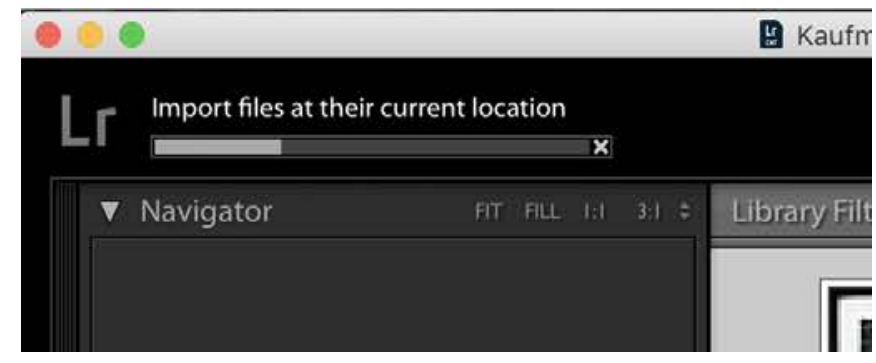

10. Once the images have finished importing, you will need to put them into a Collection. If you do not do this, all of your images will be floating around in the catalog and it will be difficult to sort and view them. You can also do this during the importing process by clicking Add to Collection on the right side of the import 
interface. You can then create a new collection or add the images to an existing one.

11. In the Collections drop down menu on the left side of the interface, click the ' + ' button.

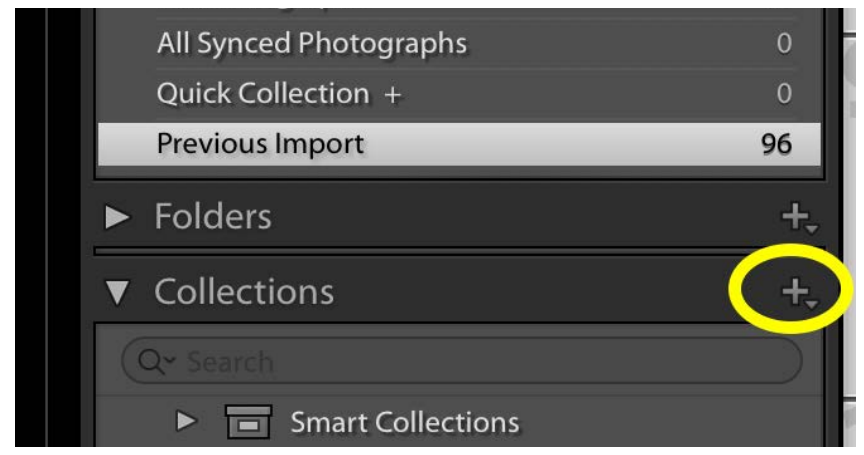

12. Next click Create Collection...

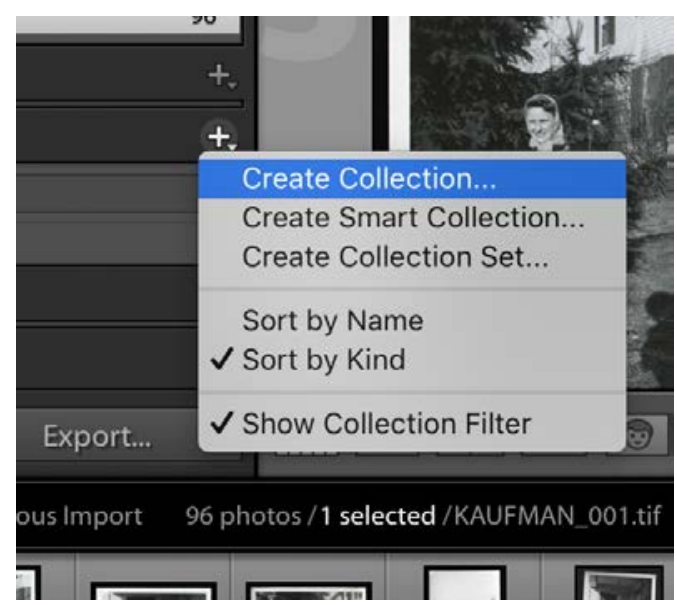

13. Name your collection, then click Create.

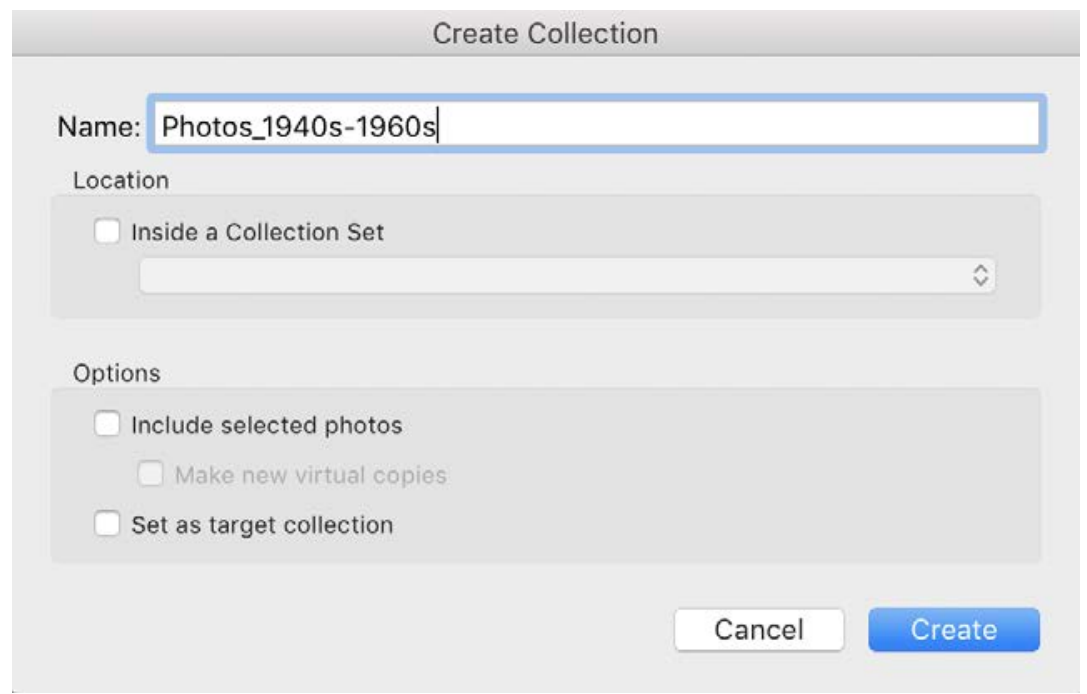


14. Your new collection will now appear under the Collections drop down menu on the left side of the interface.

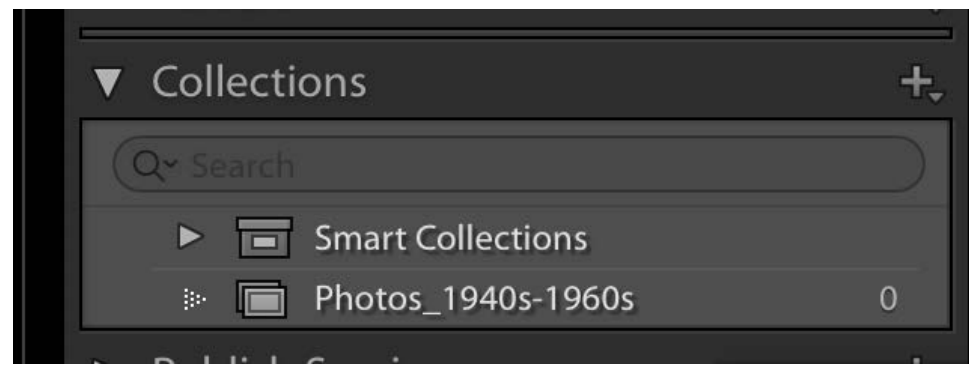

15. To add images to your new collection, select the images you have just imported. When an image is actively selected, the area around it will change from a dark to light grey.

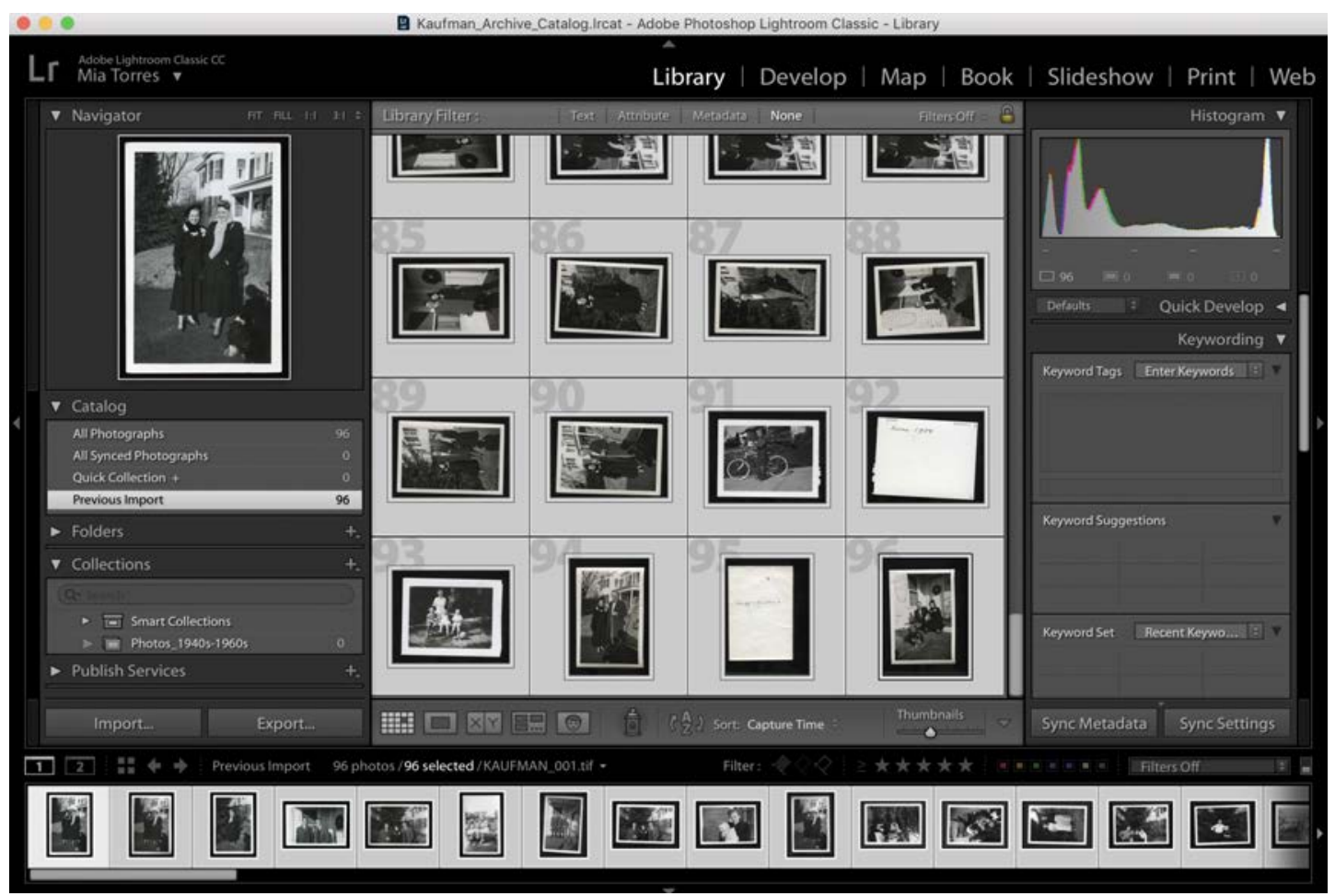


16. Next, grab any one of the images with your cursor, either from the thumbnails shown in the middle of the interface or from the strip of thumbnails along the bottom and drag it into the Collection folder. You will see a small stack of semitransparent thumbnails move with the cursor to indicate that you are moving a group of files.

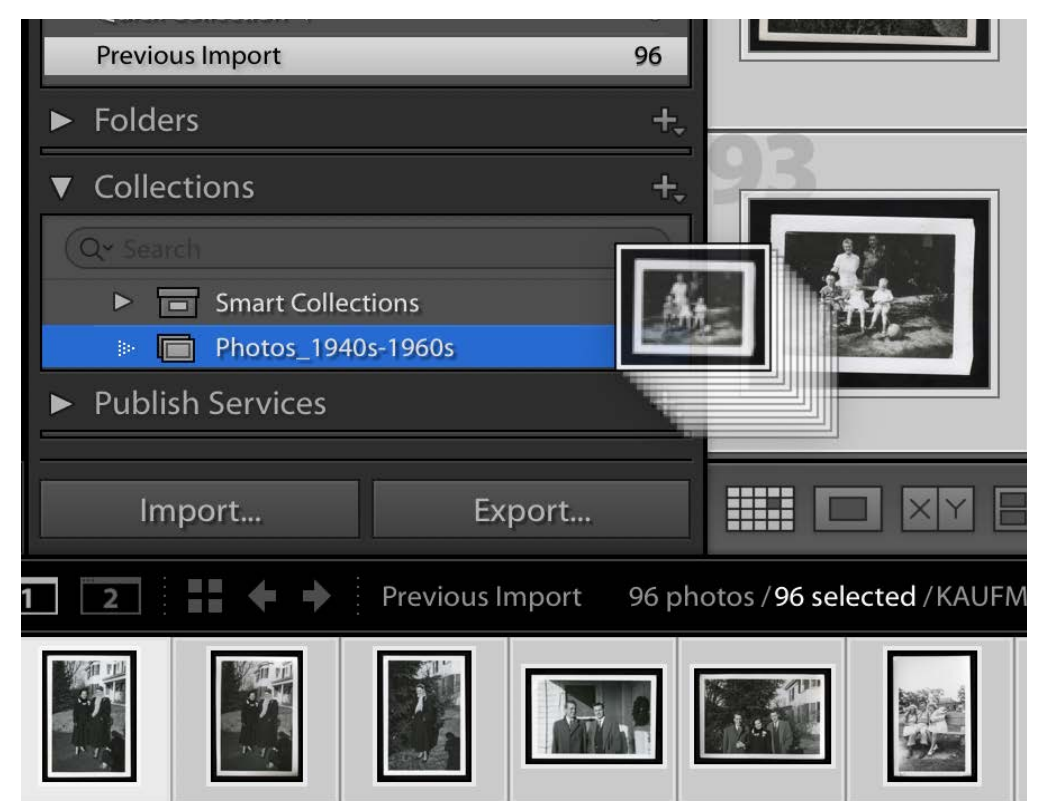

17. To deselect the thumbnails, click on the gray space around any of the selected thumbnails.

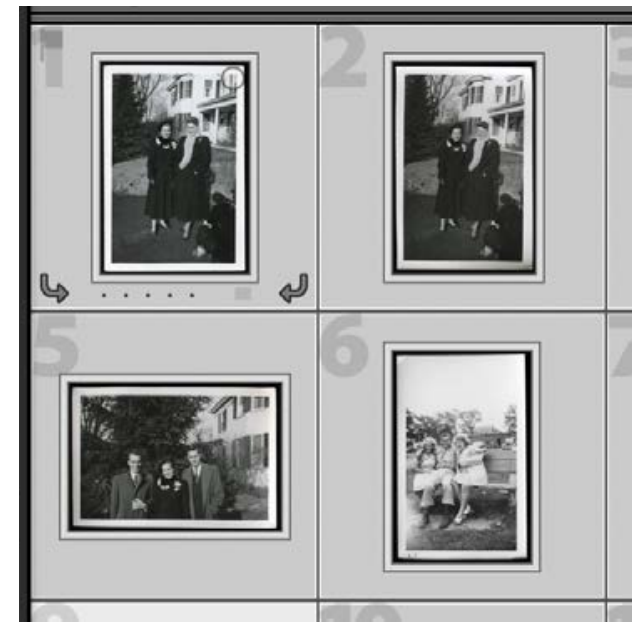

All selected

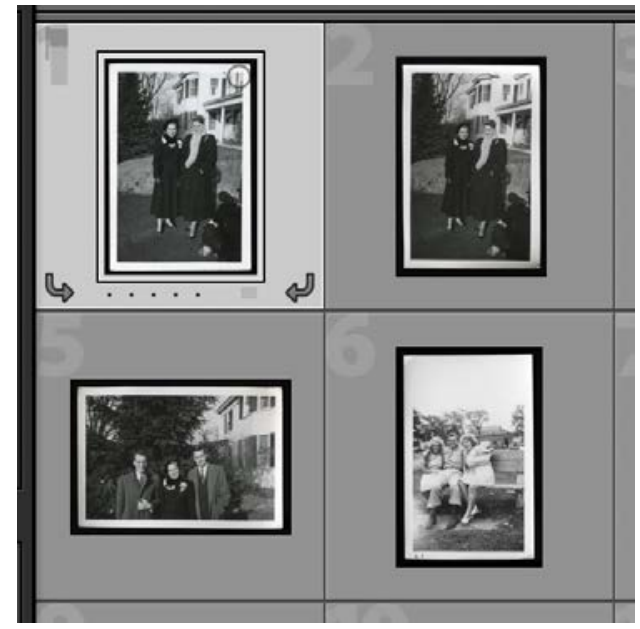

One selected 
18. If you would like to mirror the organization of your Lightroom Collections to that of the folder organization on your hard drive (which I recommend) you can create Collection Sets. A collection set is like a parent folder that can contain many subfolders, or collections.

ALBUMS
BOUND DOCS
Kaufman_Archive_Catalog
Kaufman_Inventory.xIsx
LOOSE DOCS
OBJECTS
PHOTOS

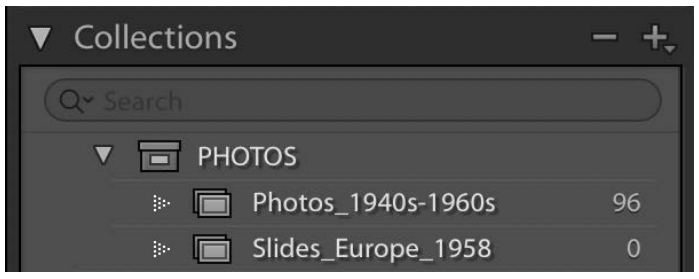

Here you can see that the folder 'Photos_1940s-1960s' is contained within the parent folder 'PHOTOS'. In order to keep everything as organized as possible in my Lightroom catalog, I like to construct a very similar-if not the same-collection and collection set structure.

19. To do this, click the ' + ' next to Collections, but this time click Create a Collection Set.

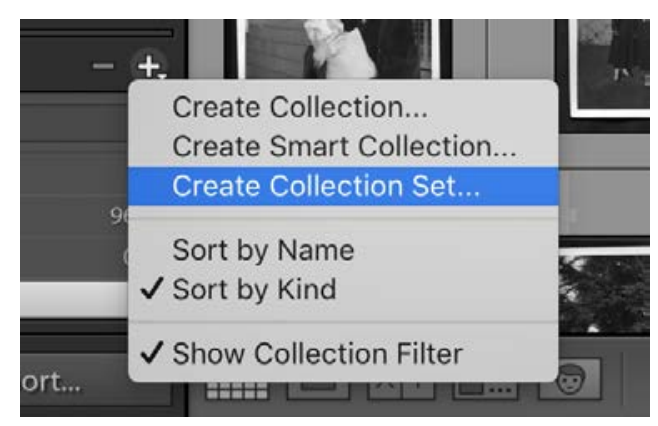

20. Name the collection set. I have chosen to name mine 'PHOTOS', which is also the name of the corresponding folder on my hard drive.

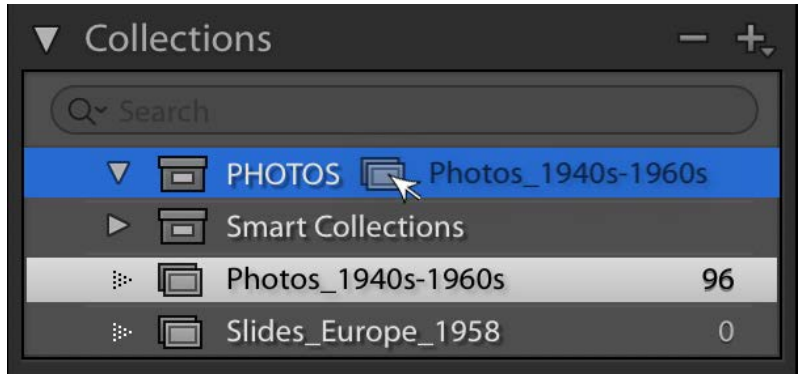


You may notice that the two collections I have created, 'Photos_1940s-1960s' and 'Slides_Europ_1958' are outside of the collection set, represented by a banker's box icon. This is an easy fix. Simply grab and drag each collection into the 'PHOTOS' collection set.

You should now see them appear under the PHOTOS collection set.
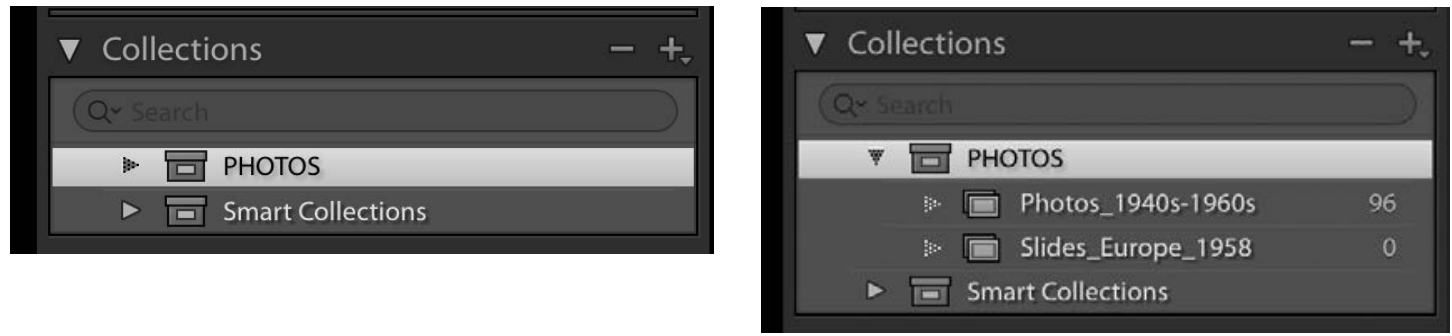

21. Now move to the Develop module by clicking DEVELOP along the top of the Lightroom window.

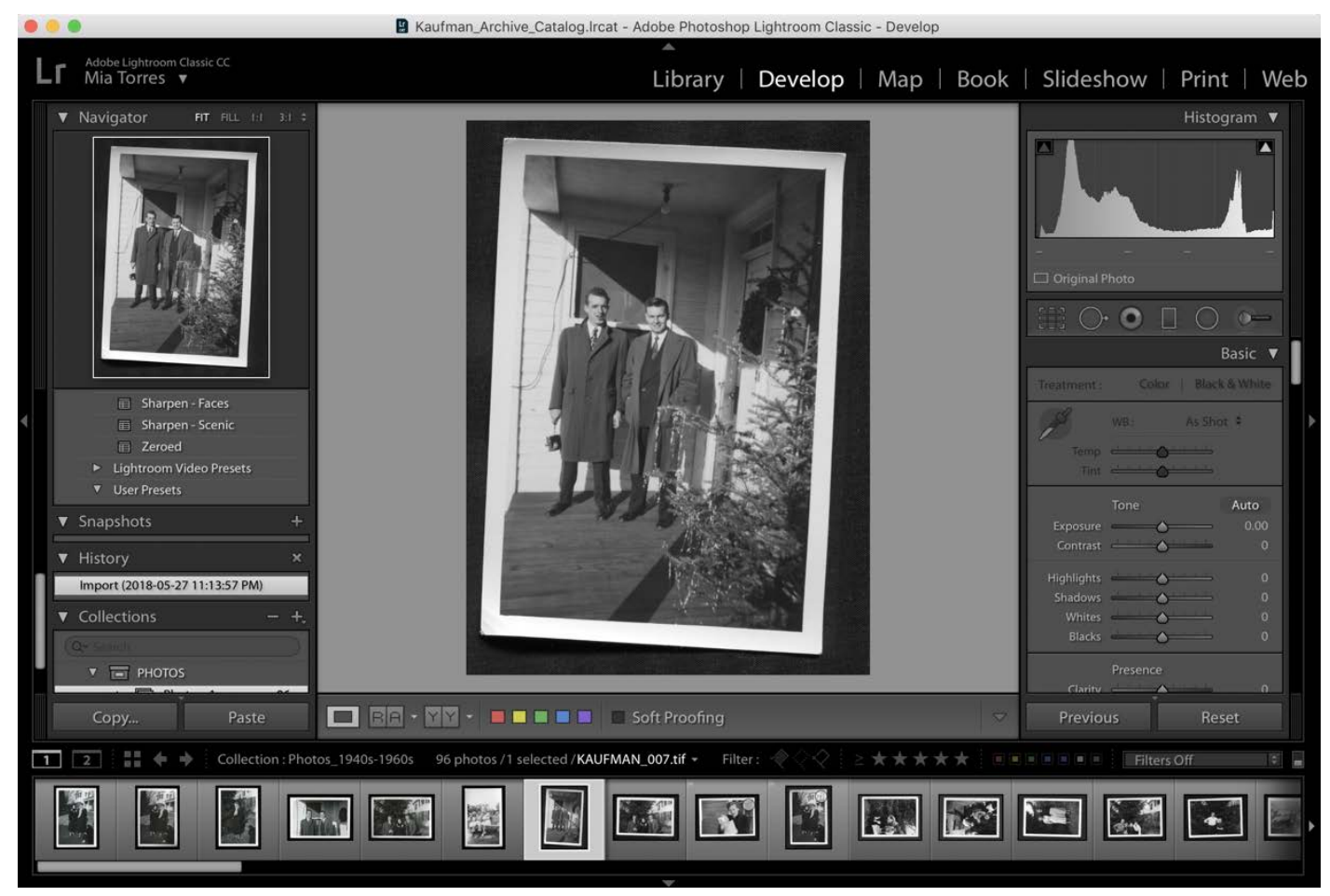


22. To de-skew (rotate) select the Crop Overlay icon to the right of the image.

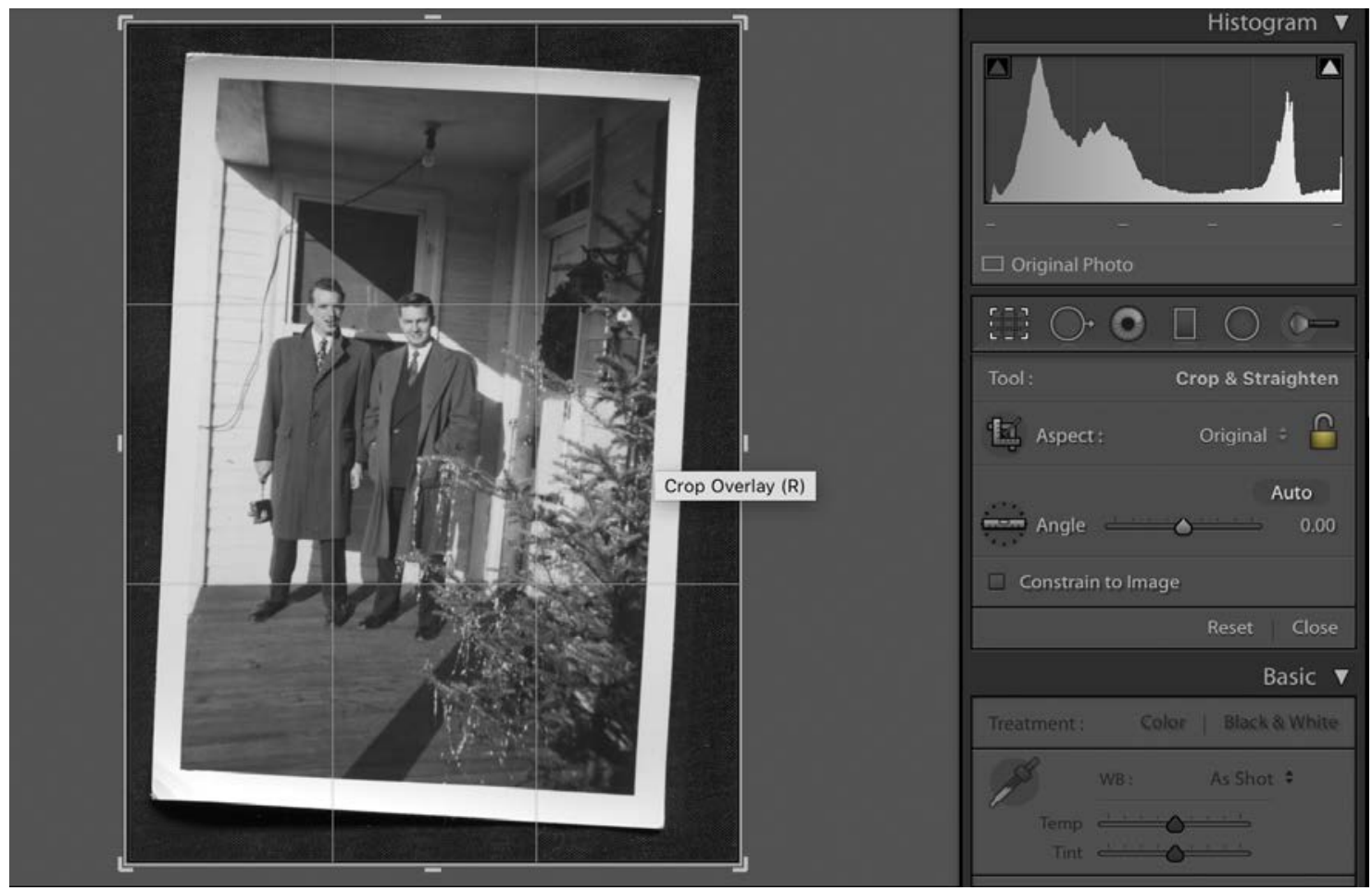

23. Rotate the image by grabbing the edge of the crop overlay with the cursor and drag until the image has been rotated to your satisfaction. Then press Enter or double click on the image to apply the crop.

NOTE: Any adjustment you make in Lightroom is not applied to the actual file on your hard drive and you do not need to save the images or the catalog after you have made adjustments to images.

24. Adjust the exposure, contrast, highlights, etc. until the image appears the way you want it to. A good rule of thumb is to look at the Histogram diagram and check to make sure none of the shadows or highlights are too far towards the edges of the diagram. This would indicate that the blacks and whites are clipping, meaning that they are so over or under exposed that you are losing pixel data (detail) in those areas. 


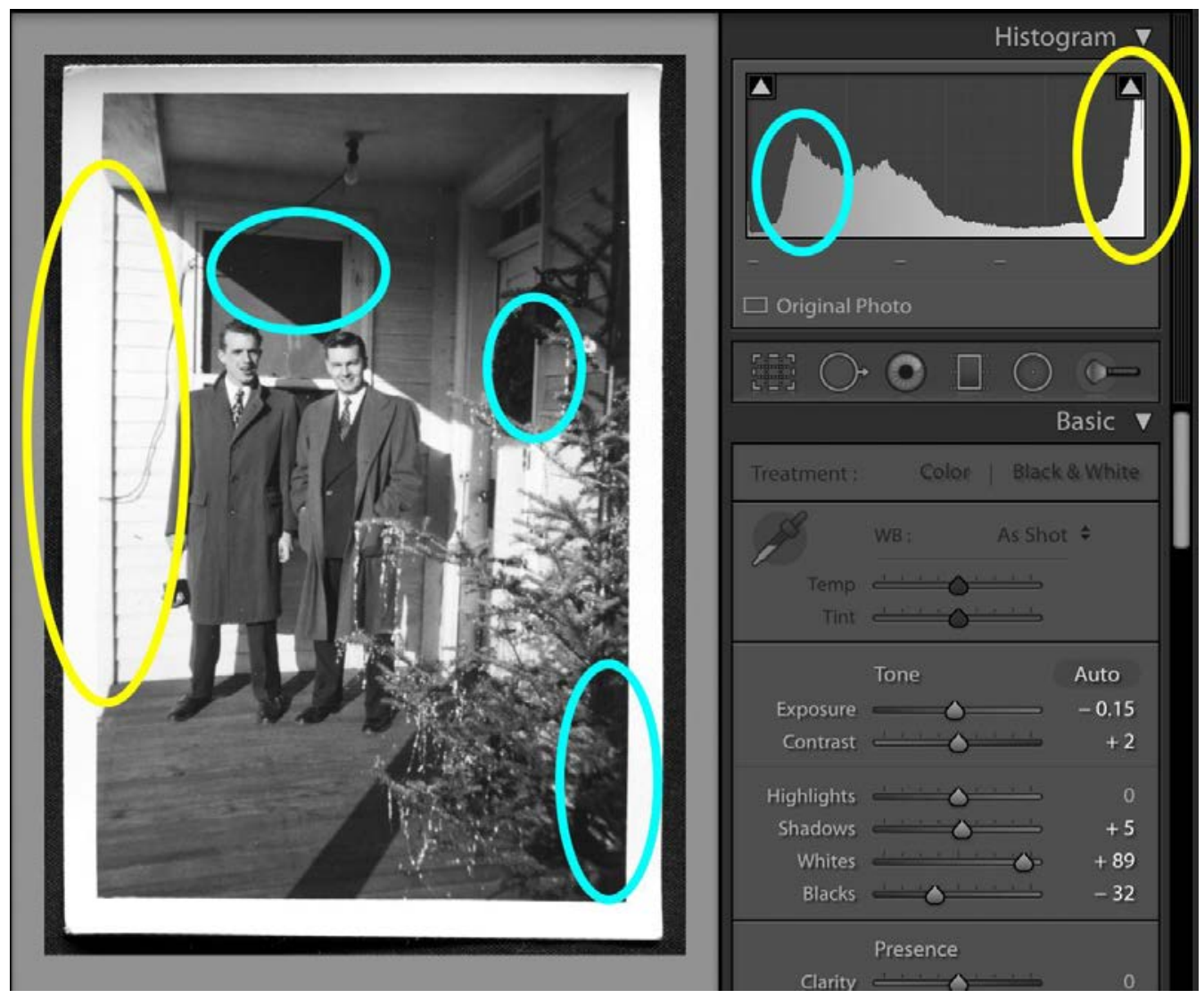

In this diagram, the yellow ovals indicate the highlight areas of the image and the teal ovals indicate the shadow areas. I have denoted the corresponding areas on the histogram. The data in the histogram represents the continuous tonal range of the image from shadows on the left side to highlights on the right side. In this image you can see that a large portion of highlight data is situated, and actually being cut off. This indicates that those highlight areas are being 'blown out'. They're over exposed so I should reduce either the exposure or the highlight values until the highlight data sits more comfortably within the histogram. If you were to lower the exposure and highlights but no detail is revealed, the area simply turns a muddy grey, this indicates that there 'is no information' in that area, meaning that the scan was over exposed. This is more common when digitizing materials with a digital camera than with a flatbed scanner. Below is a comparison between the overexposed highlights and the correct highlight tones. 

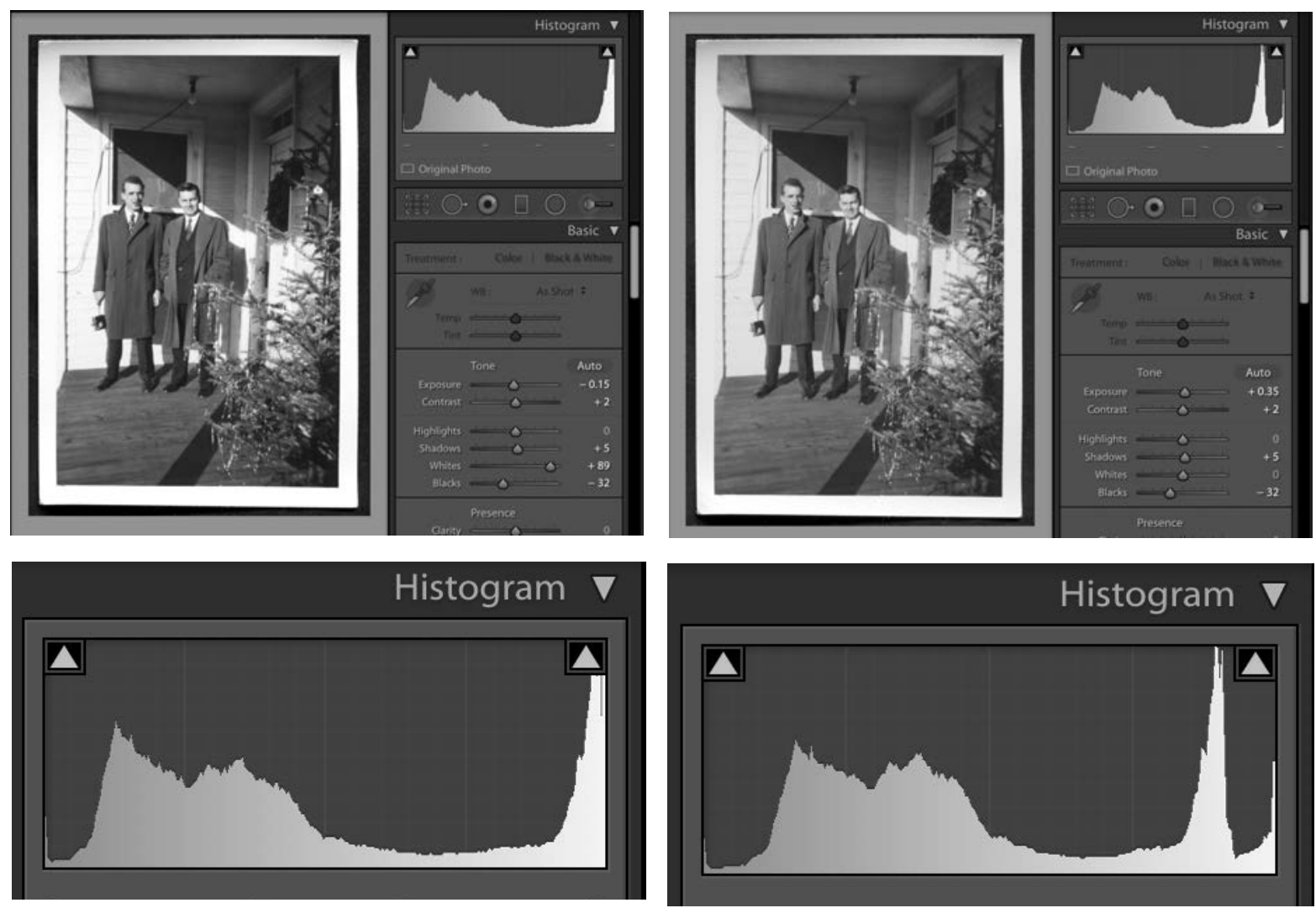

Over-exposed, clipping highlights

Correctly-exposed, highlights not clipped

25. To export the images with the adjustments you have made, return to the Library module and click EXPORT in the lower left corner of the program window (or click FILE then EXPORT). The export menu box will now appear.

26. Under Export Location you can choose where you exported files will be saved. Select Specific Folder from the Export To drop down menu. 


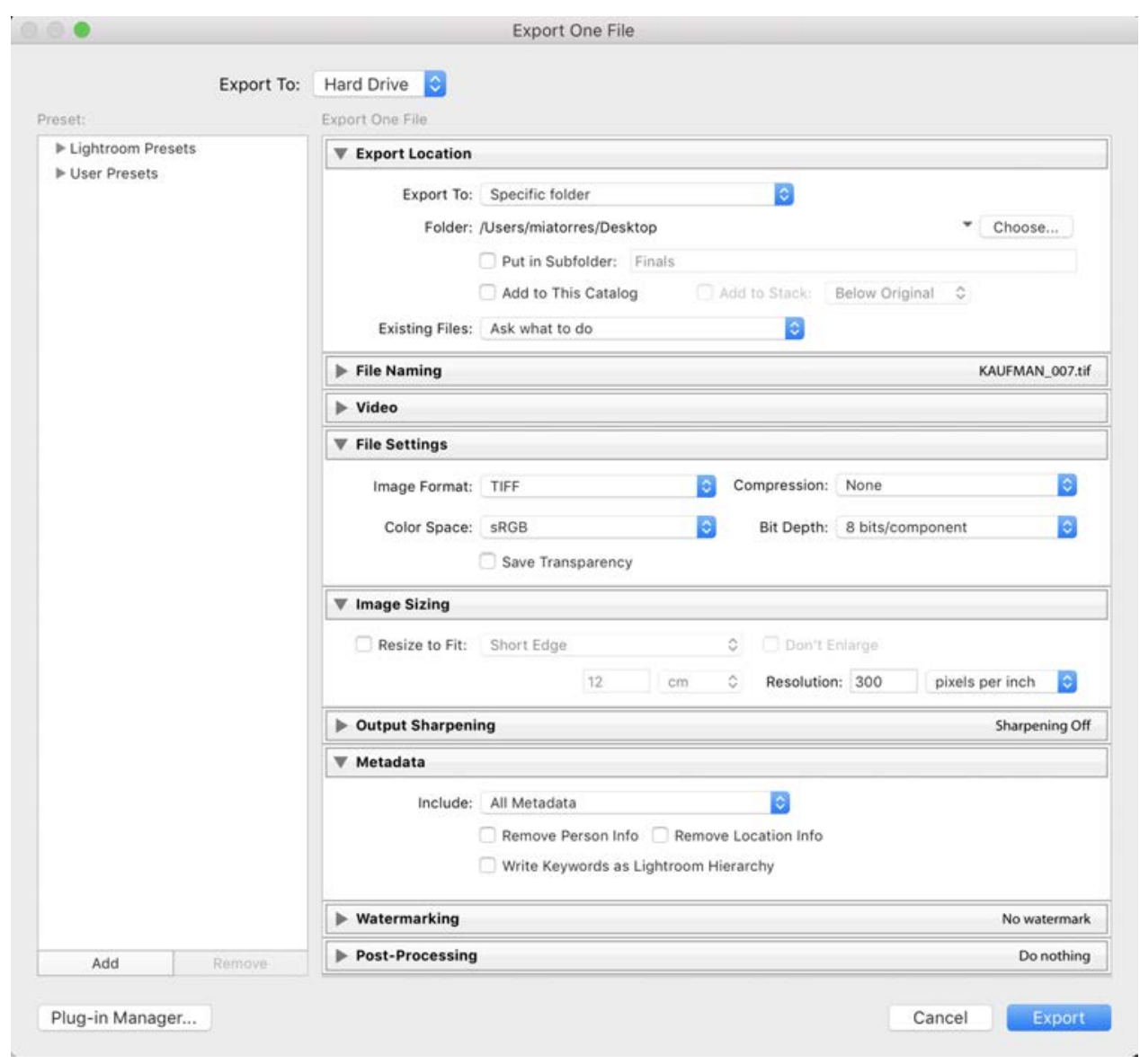

27. To specify which specific folder you would like to set as the export location, click the Choose... button. This will allow you to either select a preexisting folder or create a new one.

\begin{tabular}{l}
\hline Export Location \\
Export To: \\
Folder: /Users/miatorres/Desktop/KAUFM...tos_1940s-1960s/Production Tiffs \\
$\qquad$ Put in Subfolder: Finals Choose... \\
$\qquad$ Add to This Catalog \\
Existing Files: Ask what to do
\end{tabular}

Specific folder
Same folder as original photo
Choose folder later (useful for presets)
Desktop
Documents folder
Home folder
Movies folder
Pictures folder




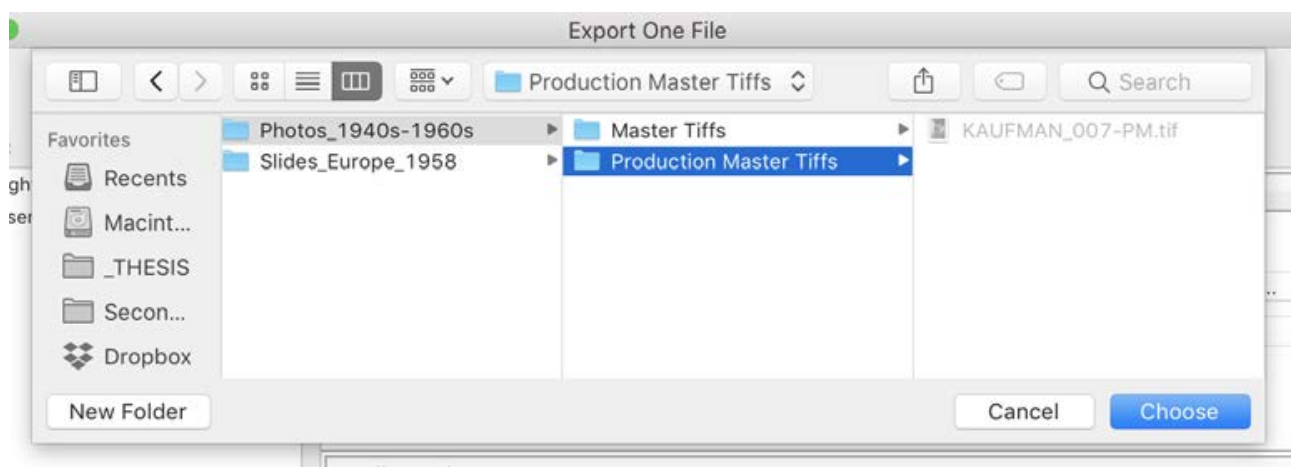

Because the images I am exporting have been edited, I am going to designate them as 'Production Master Tiffs' because they are the master files from which I will be saving all derivative files (jpegs, pdfs, and pngs). I want all of the derivative files to have the same cropping and adjustments as the edited (production) master tiff.

This designation is less important if the original file is a camera RAW format because there is no chance of the master and production master getting confused as they are different file types with different file extensions (.cr2 and .tiff for example). However, in the case of images that have been scanned as tiffs, the master and production master will have the same file name unless I add a descriptive suffix to the production master Tiff. Even though they would not be located in the same folder, things could get confusing if one of the files were to be moved. Sometimes the adjustments are not as obvious as de-skewing and cropping, so it is best to add a suffix to the new file you are exporting so it is quickly identifiable as the version with your adjustments.

However, if you would rather not have both the original and the production master tiffs, which are both large files and may not be that entirely different from each other, you can choose to overwrite the original master files. This is not compliant with FADGI recommendations, but sometimes the need to make the best use of hard drive space outweighs the need for strict adherence to a federal archival standard.

If you plan to overwrite the original masters with your edited masters, skip steps 28 and 29. 
28. This suffix can be added to the image during the exporting process. Check the box next to Rename To: and click the adjacent drop down menu. To add a custom suffix to the file (not one of the listed options), click Edit... This will allow you to create your custom naming scheme.

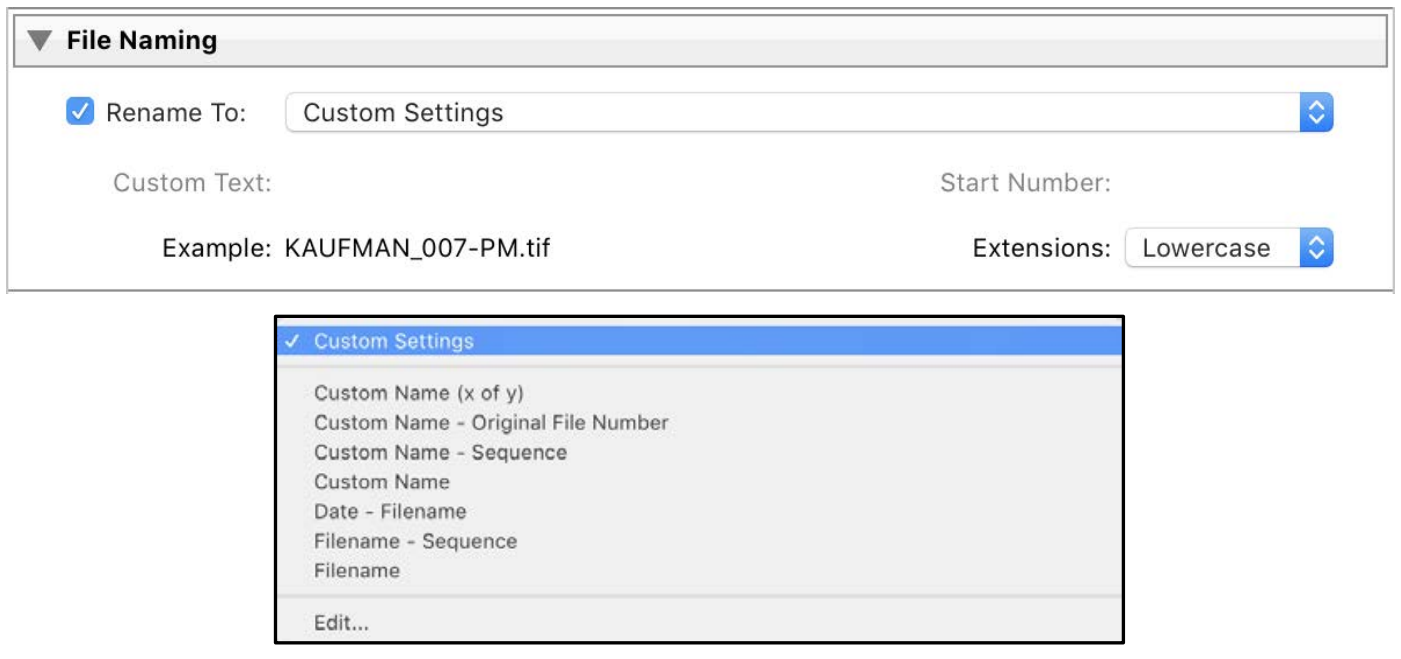

29. If not already included, add the filename to the custom naming scheme by clicking Insert next to the Filename dropdown menu. Then type either a dash (-) or underscore (_) and either 'pm' or 'PM' (or any alphanumeric suffix you would like to use) indicating that the files are 'production masters'. Because this latter edition is not highlighted blue, it is not a variable, meaning will appear the same in all exported image filenames.

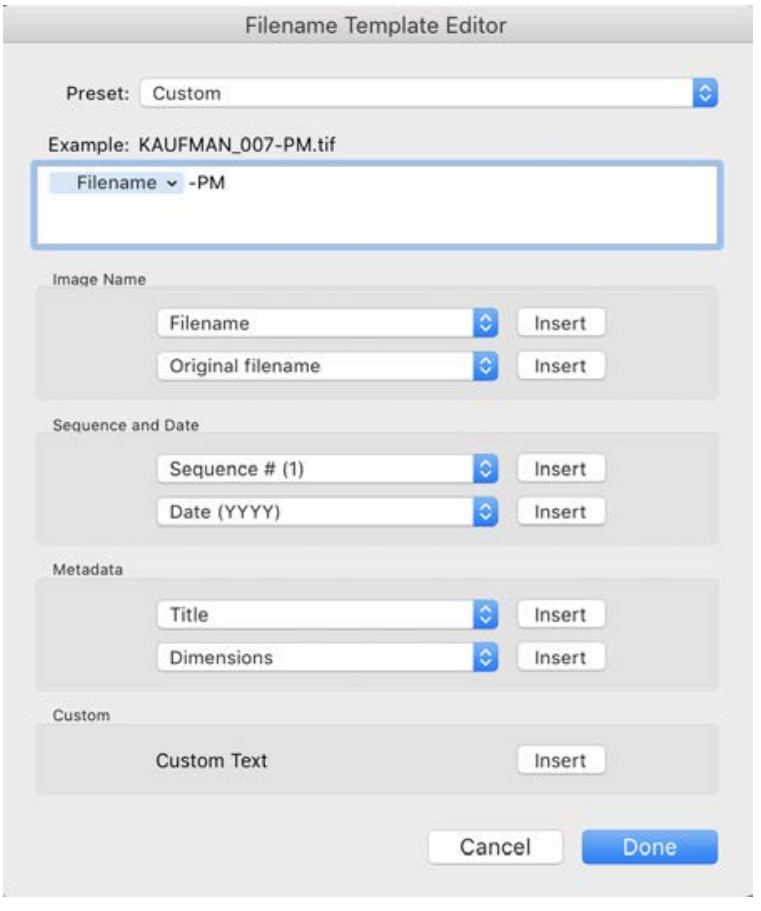

30. Then click DONE. 
31. Next, select the Image Format, Colour Space, Compression, and Bit Depth for the files to be exported.

\begin{tabular}{|l|lllll|}
\hline \hline File Settings & & & & \\
\hline \multirow{2}{*}{ Image Format: } & TIFF & $\hat{v}$ Compression: & None & $\hat{v}$ \\
Color Space: & AdobeRGB (1998) & $\hat{v}$ & Bit Depth: & 16 bits/component & $\hat{v}$ \\
& & & & & \\
& & & & \\
& Save Transparency & & & \\
\hline
\end{tabular}

\section{IMAGE FORMAT}

In most cases when exporting from the master file, you will want to export as TIFF. As discussed previously, TIFFs are uncompressed, lossless files that do not lose any data when edited and saved. However, if for some reason you are exporting from a JPEG original, it is not recommended that you export a TIFF from that JPEG because you are in essence asking the computer to generate pixel data in order to create a higher quality file. Programs are getting better at generating this data, called 'upsampling' or 'resampling', but are still nowhere near as accurate as an original TIFF file would be. If the original file is either a RAW format or a TIFF, exporting a derivative TIFF is fine.

\section{COMPRESSION}

Select None unless LZW is an available option. LZW is a lossless compression meaning it will not get rid of any pixel data when compressing the image. I almost always export TIFFs with LZW compression when I am able because it does not reduce the quality or jeopardize the potential longevity of the file but does create a smaller file, saving hard drive space.

\section{COLOUR SPACE}

Just as you should not export a TIFF from a JPEG original, you should not export a file with a larger colour space than the colour space of the original. In order from smallest to largest the colours space options provided in Lightroom are: sRGB, Display P3, Adobe RGB (1998), and ProPhoto RGB. So, if your original file (the file from which you are 
exporting) is in Adobe RGB (1998), you should not export as ProPhoto RGB because the computer will have to generate colours that may or may not be accurate. If your original file is in sRGB, you should only export as sRGB. For more information about colour spaces, please refer to Section 4.4.

\section{BIT DEPTH}

When exporting a production master file or a new version of the master that will overwrite the original, you want to maintain the largest bit depth possible, meaning you should match the bit depth specified during export to the bit depth of the original file. Because I scanned these images as 16-bit grayscale, I will also export at 16-bit.

32. Under the Image Settings menu, you can specify the dimension you want the exported files to have, if different than the original, as well as the resolution you want the exported file to have. If you are creating a production master, you want the export resolution to be the same as the original master, in this case $1200 \mathrm{dpi}$.

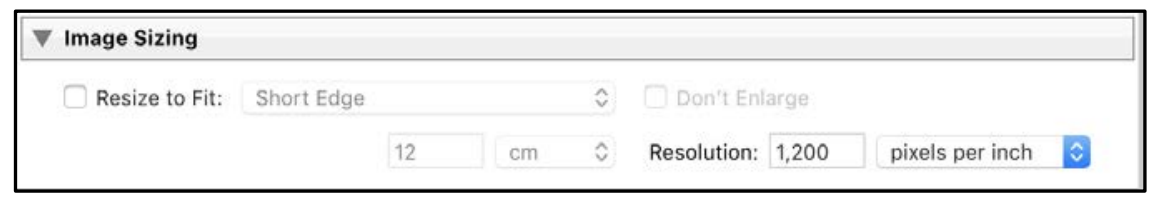

33. Finally, if you have added metadata to the files, you can specify which parts of the metadata you want to embed in the exported files.

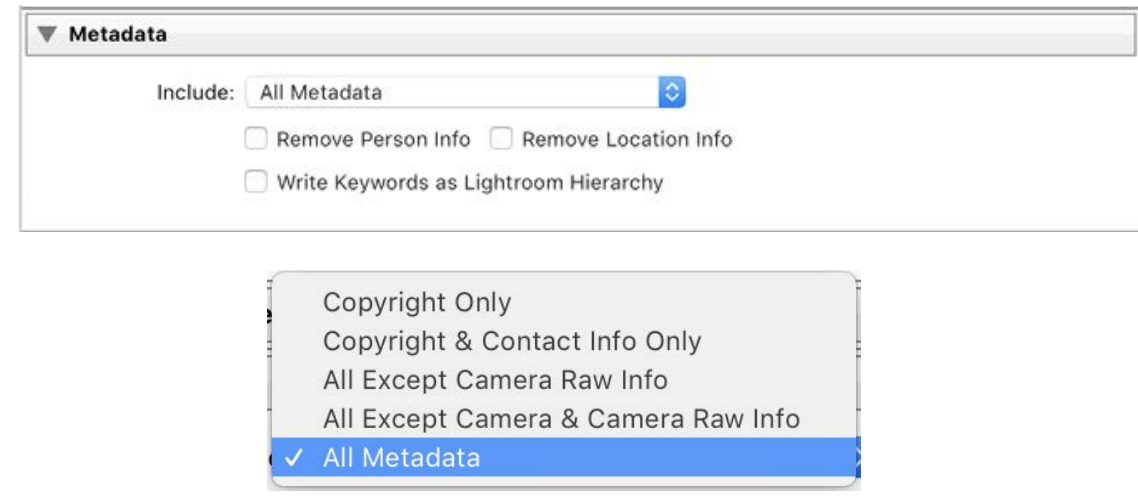


34. Once you have finished customizing the export settings, click Export. You may be asked to select the destination folder again. Once you have navigated to the folder, click Open.

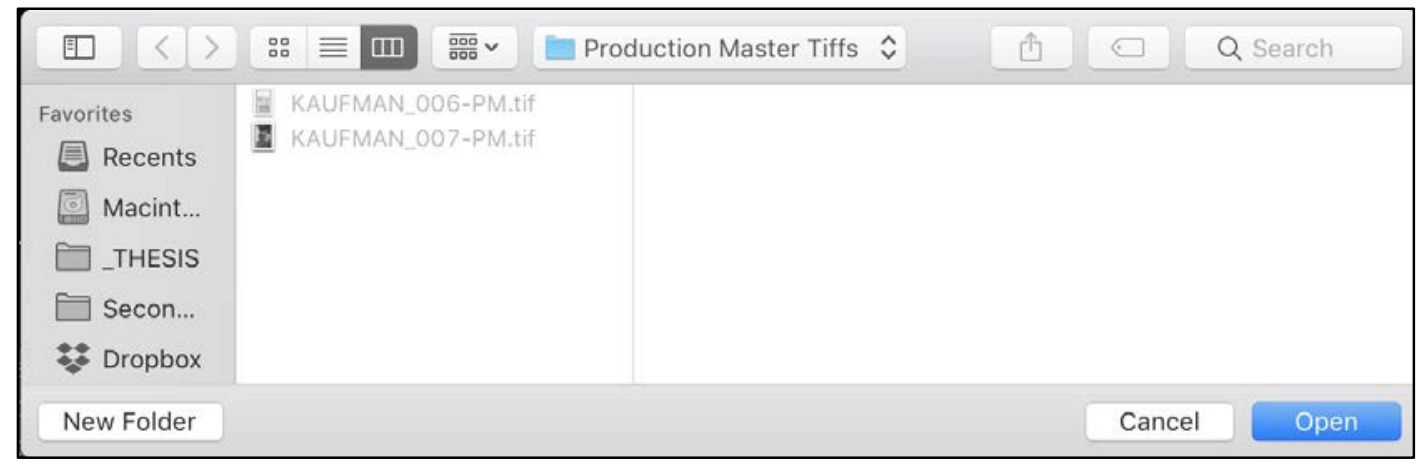

35. Lightroom will now export the file or files you have selected. Depending on how many there are, as well as the speed of your computer's internal processor, this can take anywhere from a fraction of a second to several minutes or more.

36. You can now close Lightroom. You may be asked if you would like to back up the catalog. This is good to perform after every few times you open the catalog. 


\subsection{Editing Images In Gimp}

1. Open Gimp (version 2.8 for Mac and 2.10 for Windows).
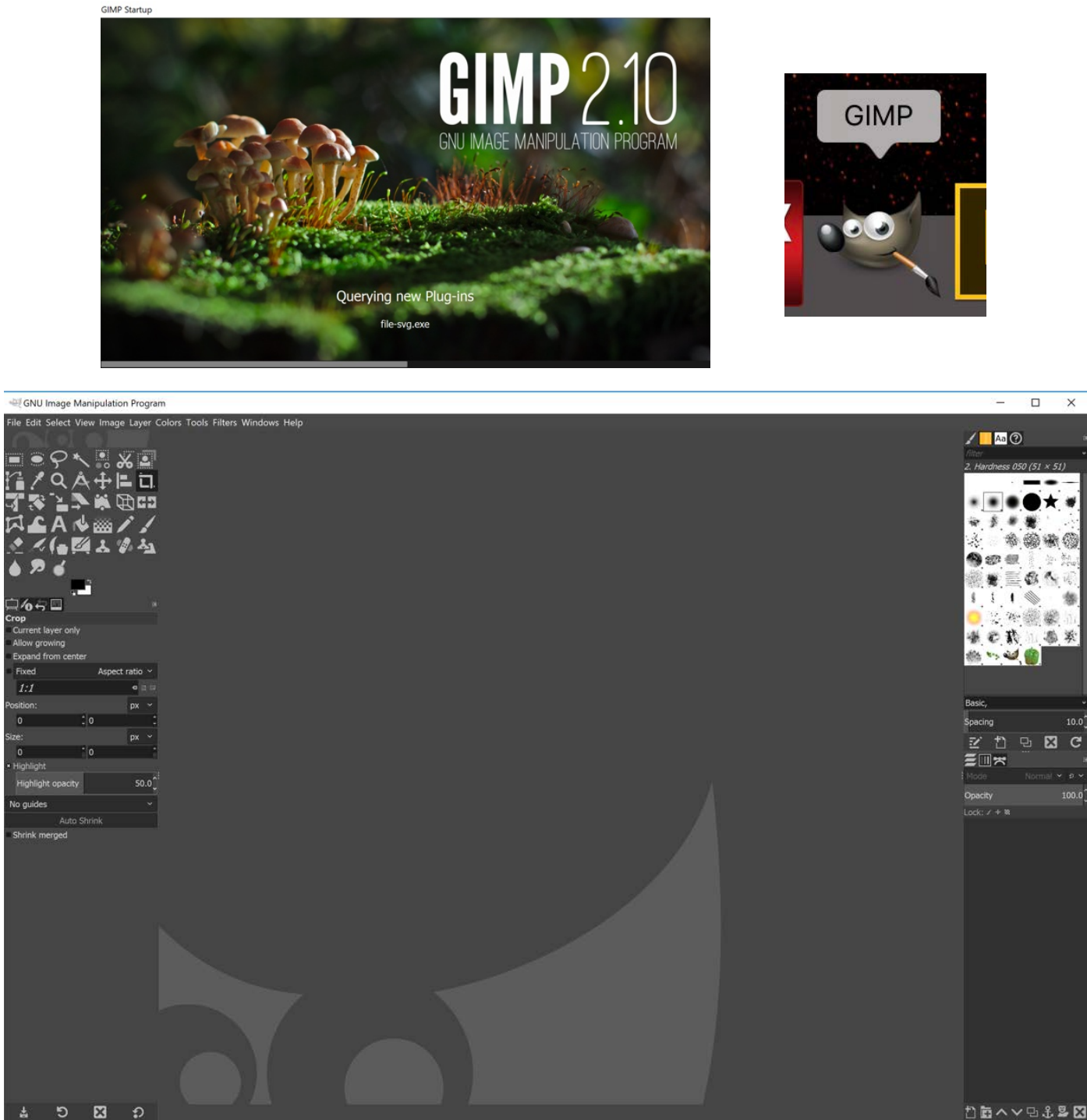

2. To open an image, go to FILE > OPEN or press Control + $\mathbf{O}$.

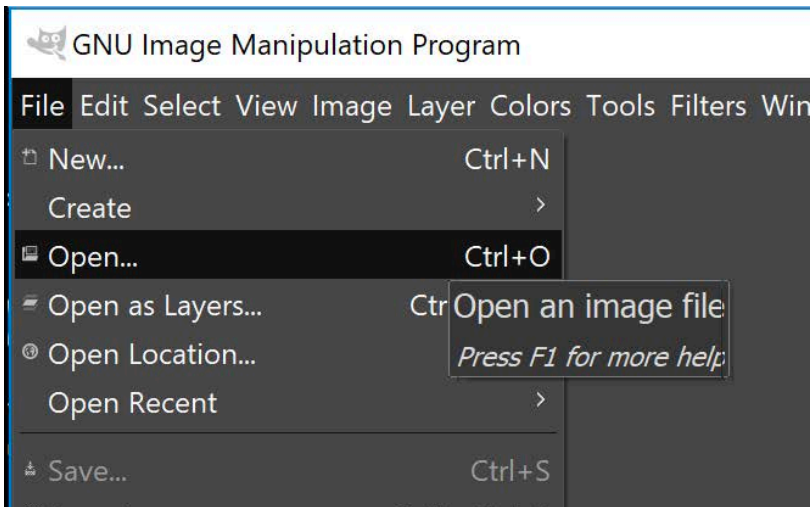


3. In the window that opens, use the panel on the left side to navigate to the folder containing the image you want to open.

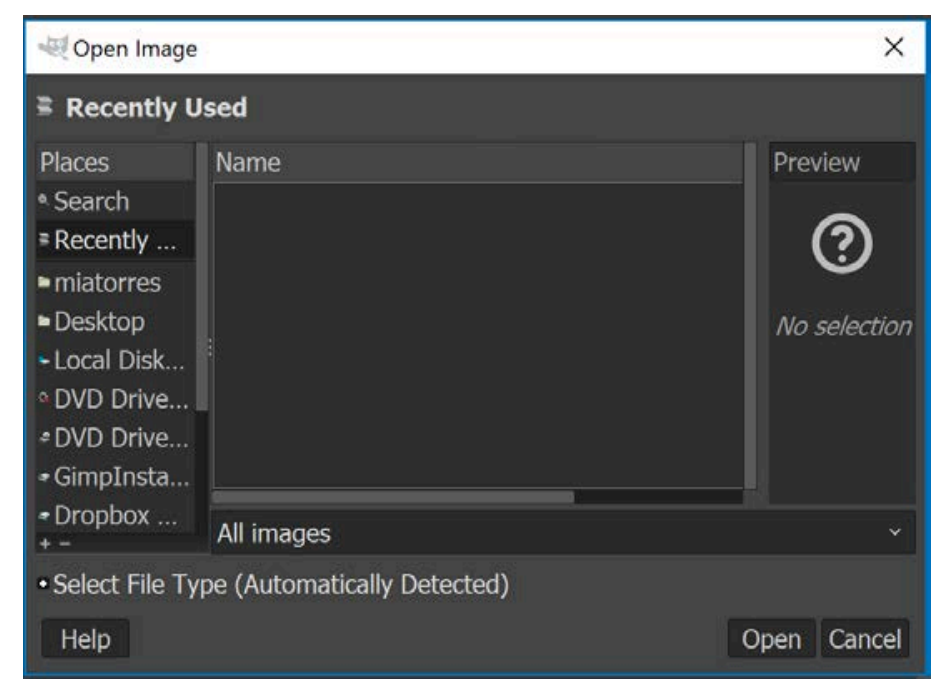

4. The folder path will be shown along the top of the window. Below you can see Desktop > KAUFMAN_ARCHIVING > PHOTOS > Photos_1940s-1960s > Master Tiffs. Click on the file you want to open and click OPEN. Make sure that "All Images" is selected in the dropdown menu in the lower section of the window. If not, you will only be able to see the file type that is selected in that dropdown menu.

\begin{tabular}{|c|c|c|c|c|c|c|}
\hline \multicolumn{3}{|l|}{ Open Image } & & & & $\times$ \\
\hline Home - Desktop & sktop KAUFMAN_ARCHIVING & PHOTOS & Photos_1940s-1960s & \multicolumn{2}{|c|}{ Master Tiffs } & \\
\hline Places & Name & & ^ Size & Modified & \multicolumn{2}{|c|}{ Preview } \\
\hline - Deckton & - KAUFMAN034.tif & & $92.9 \mathrm{MB}$ & 2018-04-01 & \multirow{2}{*}{\multicolumn{2}{|c|}{$m>$}} \\
\hline -local Disk & "KAUFMAN035.tif & & $83.4 \mathrm{MB}$ & 2018-04-01 & & \\
\hline DVD Drive. & -KAUFMAN036.tif & & $81.7 \mathrm{MB}$ & 2018-04-01 & & \\
\hline -DVD Drive... & a KAUFMAN037.tif & & $67.6 \mathrm{MB}$ & 2018-04-01 & \multicolumn{2}{|c|}{ KAUFM...037.tit } \\
\hline - GimpInsta... & AKAUFMAN038.tif & & $71.0 \mathrm{MB}$ & 2018-04-01 & \multirow{3}{*}{\multicolumn{2}{|c|}{$\begin{array}{c}67.6 \mathrm{MB} \\
4856 \times 4637 \text { pixels } \\
\text { RGB, } 1 \text { layer }\end{array}$}} \\
\hline -Dropbox ... & - KAUFMAN039.tif & & $81.1 \mathrm{MB}$ & $10: 39$ & & \\
\hline - iCloud on ... & - KAUFMAN040.tif & & $67.6 \mathrm{MB}$ & 2018-04-01 & & \\
\hline - Home on '... & -KAUFMAN041.tif & & $71.0 \mathrm{MB}$ & 2018-04-01 & & \\
\hline - Pictures & - KAUFMAN042.tif & & $73.3 \mathrm{MB}$ & 2018-04-01 & & \\
\hline Documents & - KAIIFMANO43 tif & & $73.9 \mathrm{MR}$ & $2018-n 4-n 1$ & & \\
\hline & & All inlages & & & & 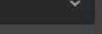 \\
\hline \multicolumn{7}{|c|}{ - Select File Type (Automatically Detected) } \\
\hline Help & & & & & Open & Cancel \\
\hline
\end{tabular}


5. The file you have selected will now open.

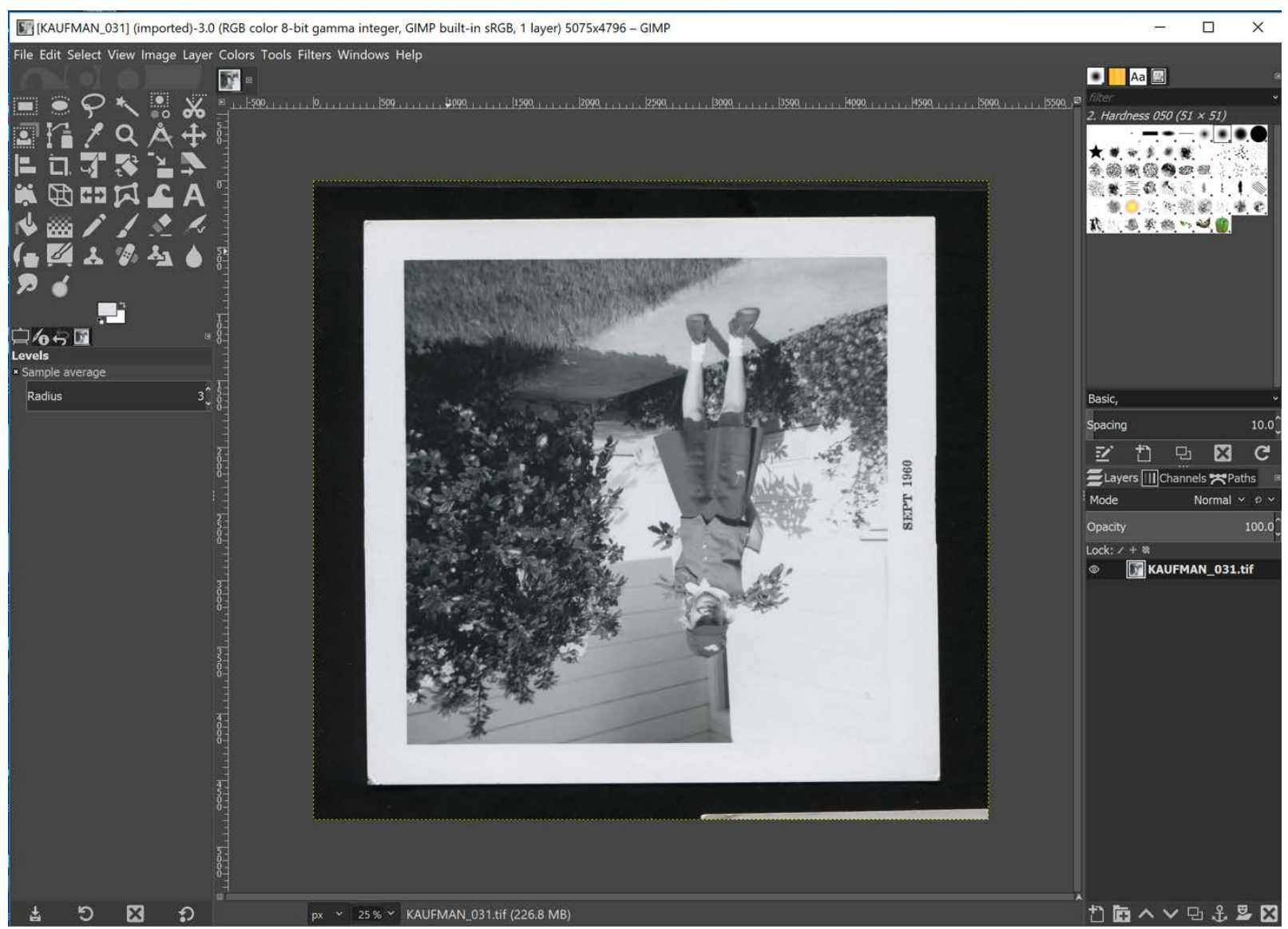

6. To ROTATE the image, click the rotate icon in the left side tool panel.
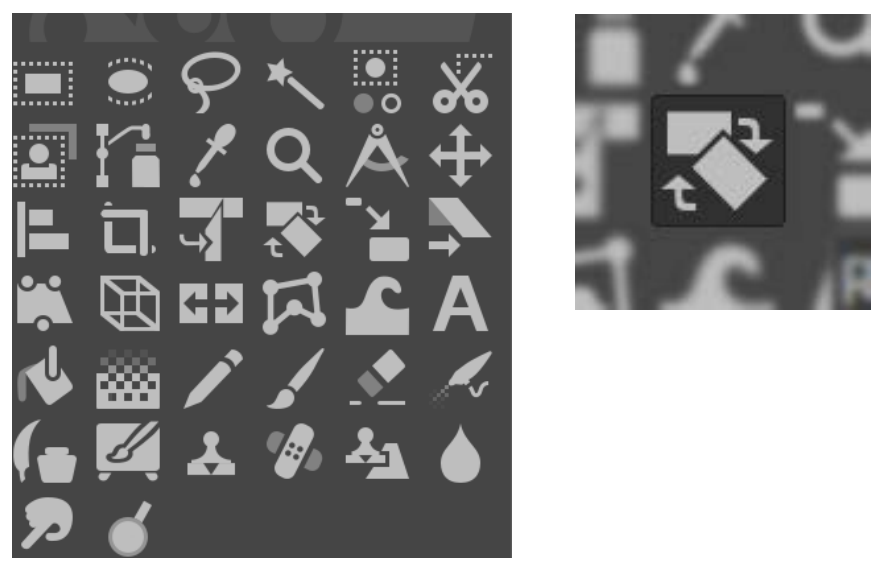
7. You will now see a thin yellow dotted line appear around the edge of the image. With your mouse, and click and hold any point along the dotted line and drag your mouse the rotate the image. Once you have adjusted it to the desired angle, release the mouse and hit ENTER.

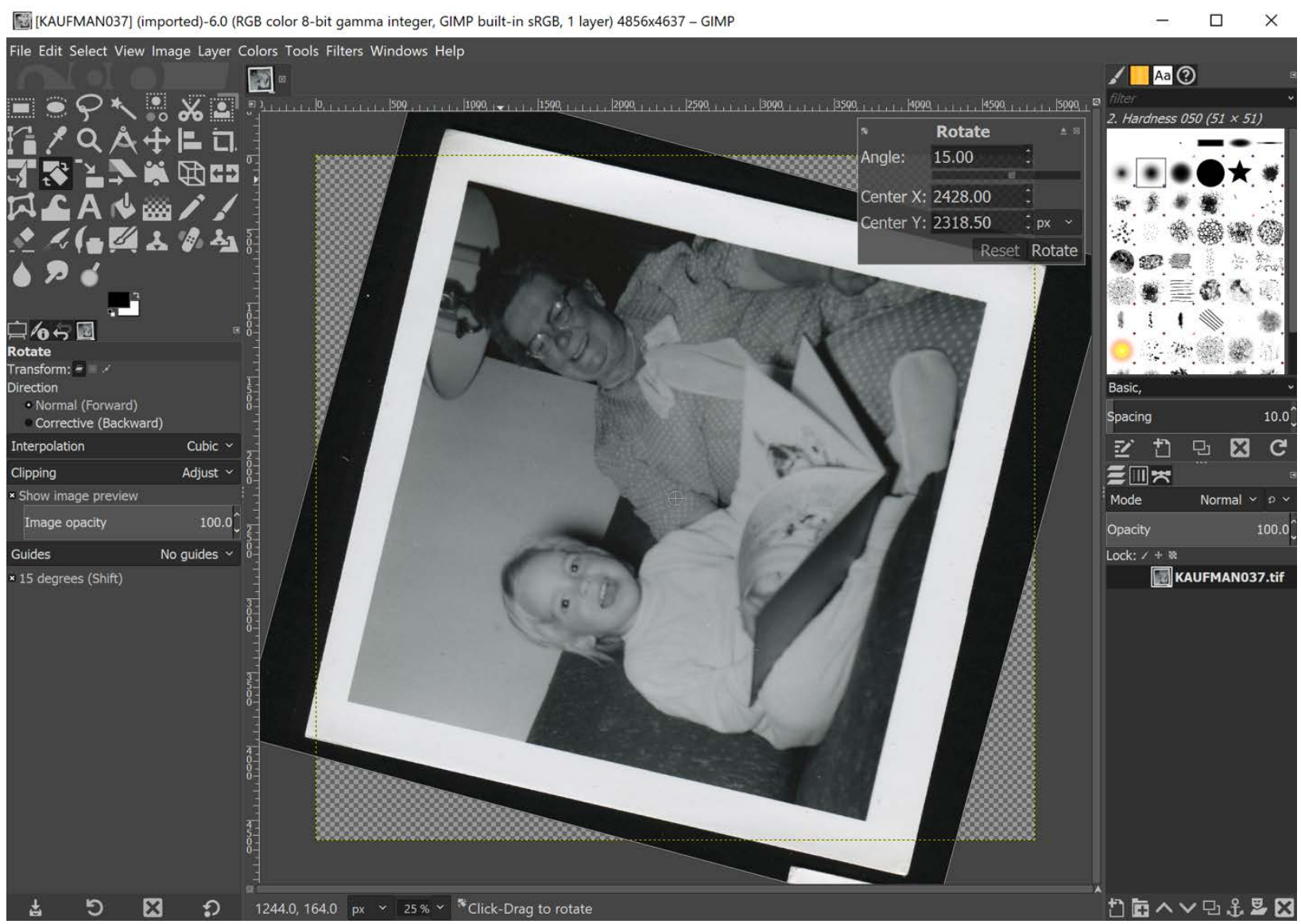

You can also rotate the image by a specific angle by typing a numeric value into the small menu in the upper right corner of the image canvas area.
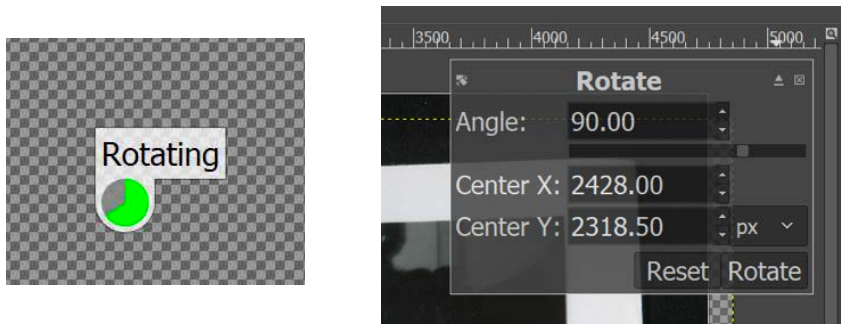
8. To crop the image, select the CROP tool from the left side tool menu.

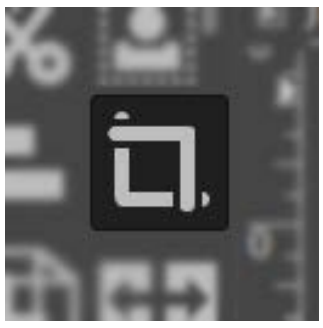

9. Click an area in a corner of the image, hold, and drag your cursor to draw a crop area around the image. To adjust the crop click and hold a section of the cropping frame and move to the desired position.

10. To apply the crop to the image, press ENTER.
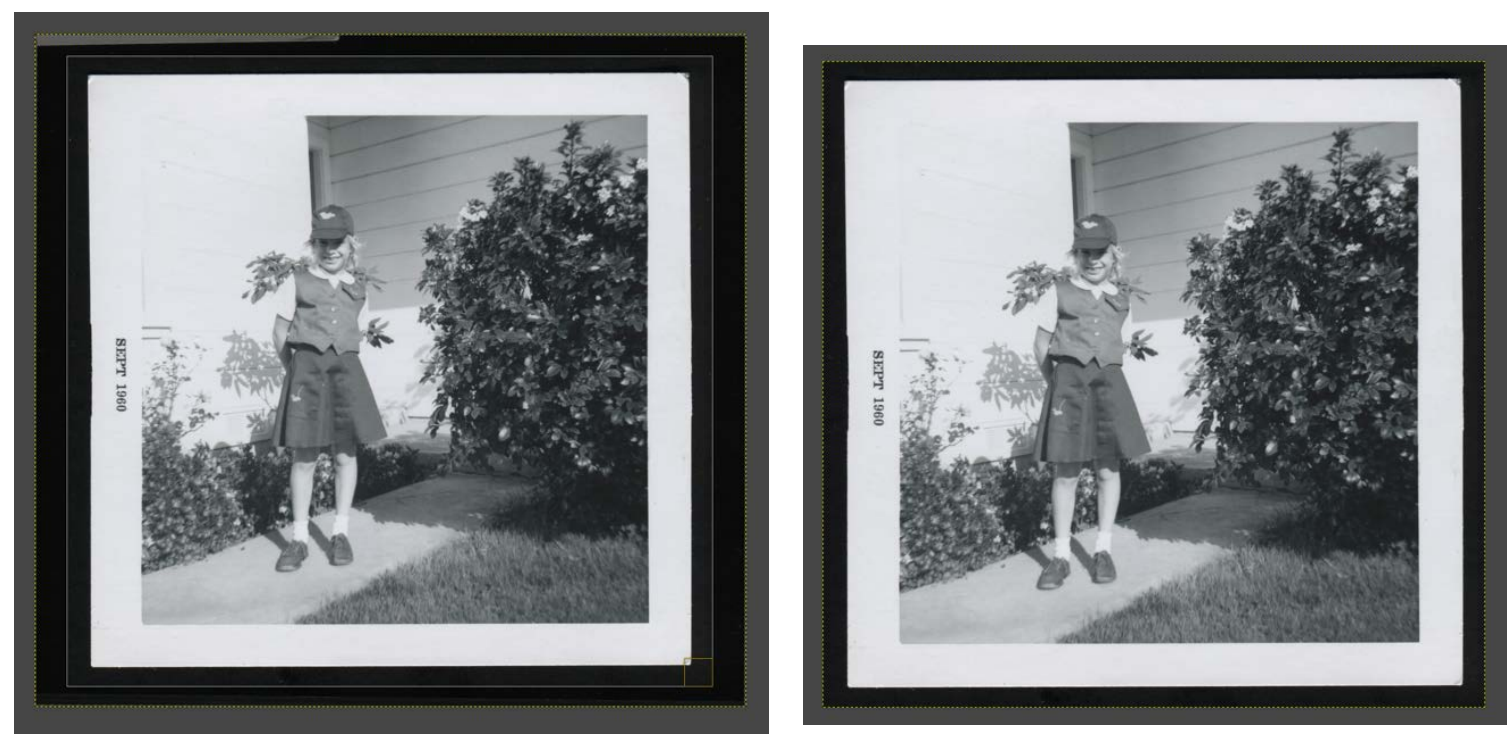

11. In the following image, you can see that the edge of another scanned photograph is visible along the edge of the image. It is too close to crop, so to remove it, I am going to paint over it with a colour that matches the dark frame around the image. 


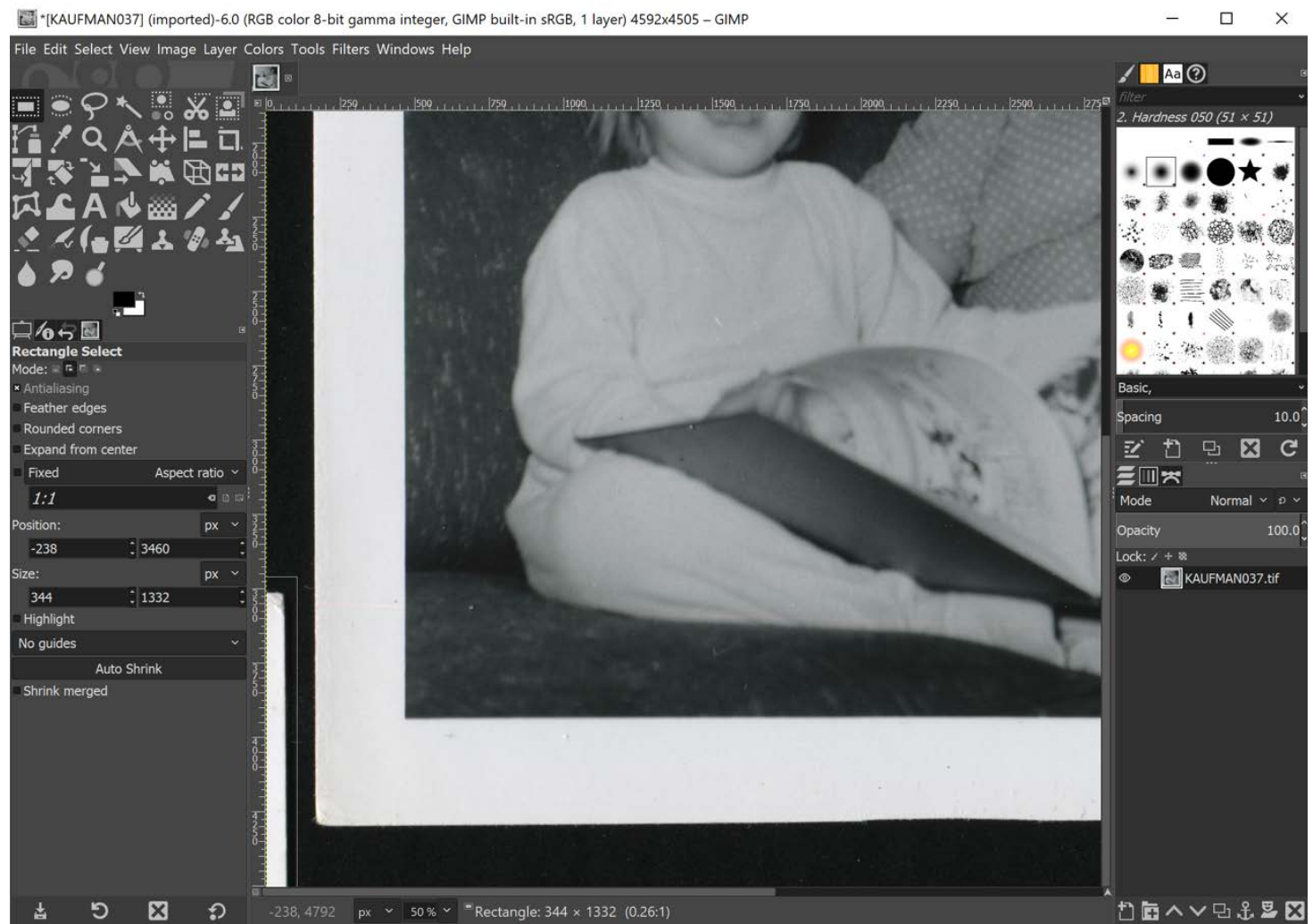

12. Click the SELECT tool in the left side tool menu.

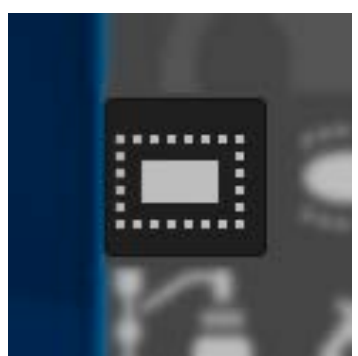

13. With the select tool selected, use your cursor to draw an area around the section of the image you want to fill in. Selecting the area you want to fill in will restrict any filling in you to do that area, so it will not spill over onto the photograph's white boarder.

14. Now that the area is selected, select the PAINTBRUSH tool from the left side tool menu.

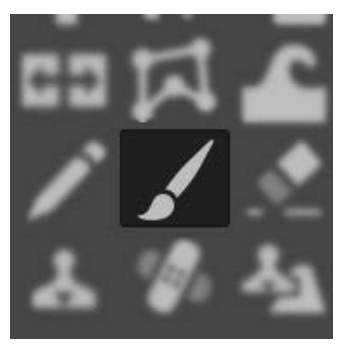


15. Next, click the small rectangular swatch of colour located just below the left side tool menu. You will see two rectangular swatches, one slightly under the other. These indicate the foreground and background colours. The colour that will be applied by the paintbrush is the foreground colour. Some

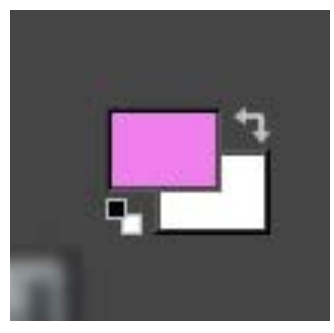
other functions, like rotating the canvas, will use whatever the set background colour is to fill in the new space around the canvas created by the rotated image.

16. To change the foreground colour, click on the top colour swatch. The 'change foreground colour' menu will now appear.

17. To match the colour swatch to the background colour (the colour that you want to use to paint over the area you selected), select the EYEDROPPER tool located to the left of the HTML notation alphanumeric box and click a border area of the image to select the colour.

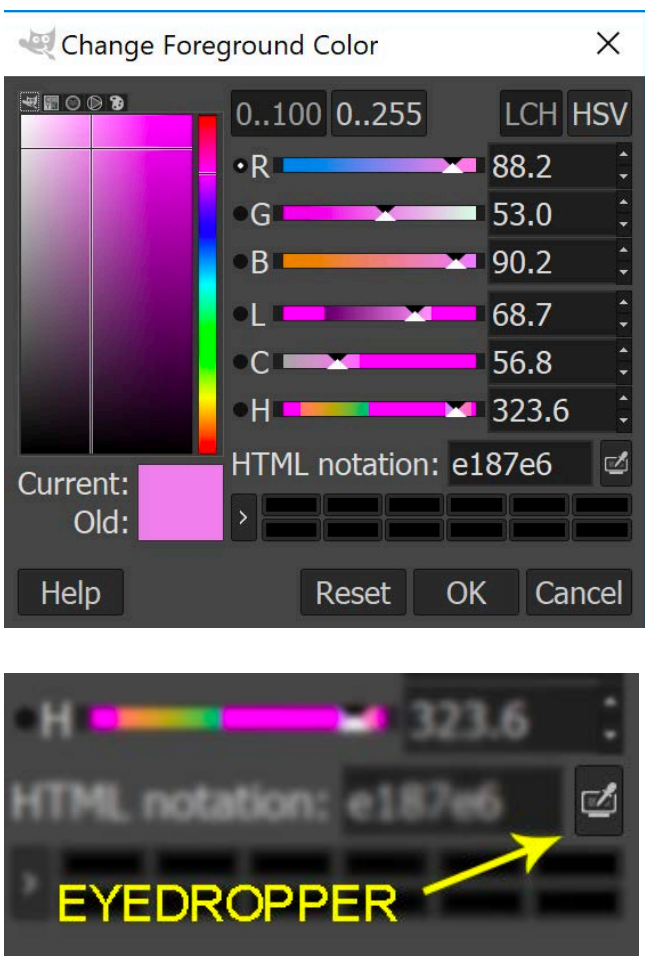

18. You will now see the CURRENT colour swatch change in the 'change foreground colour' menu.
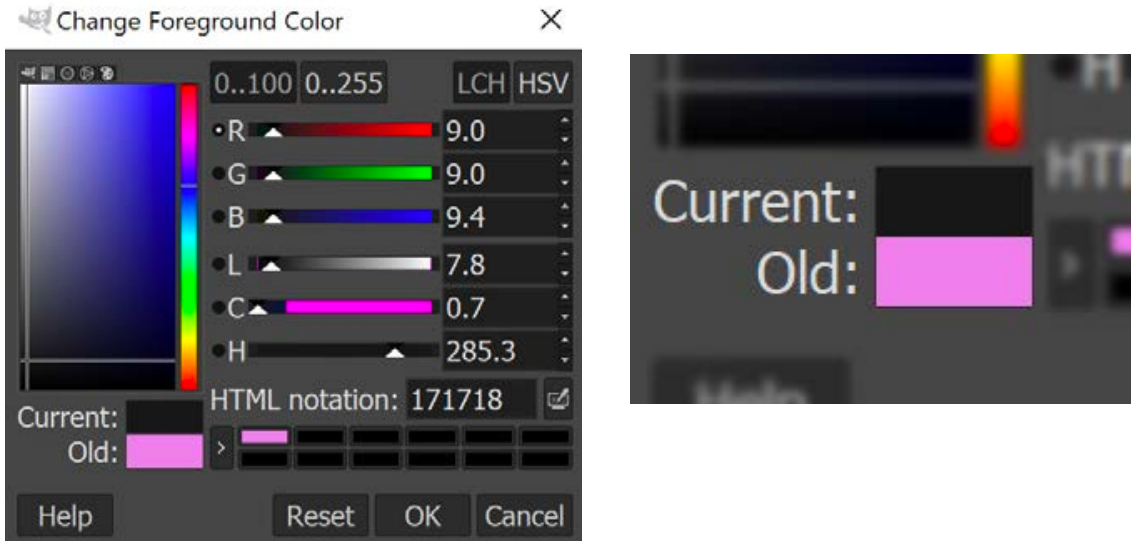
19. Click OK to choose your colour selection.

20. Use your cursor, now the paintbrush tool, to paint over the selected area. To change the brush size, shape, edge hardness, etc. use the adjustment menu that appears in the left side toolbar menu when the paintbrush is selected.
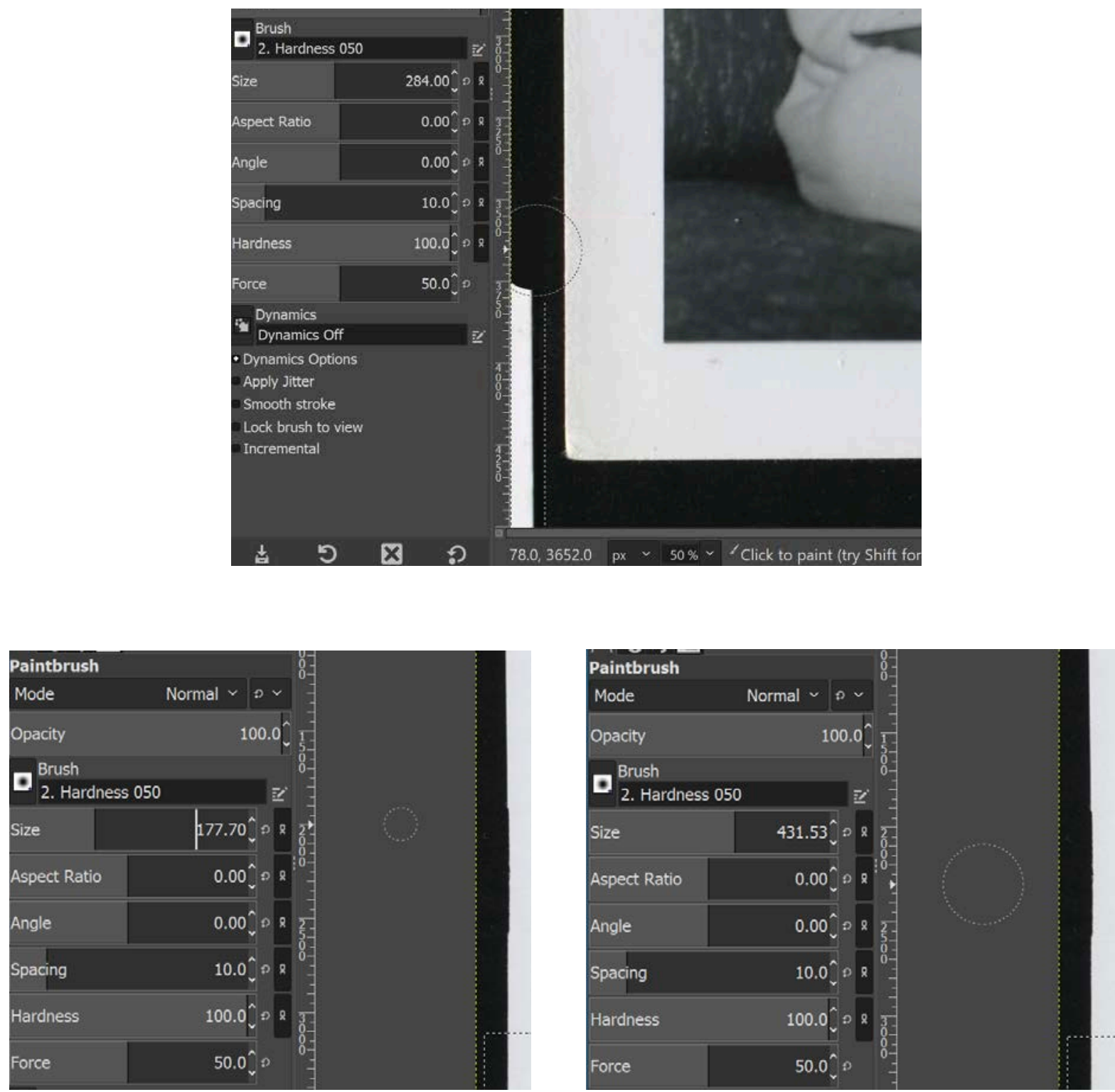
21. To check the levels of the image to make sure that no values are over 245 or under $10^{55}$, go to COLORS > INFO > HISTOGRAM.

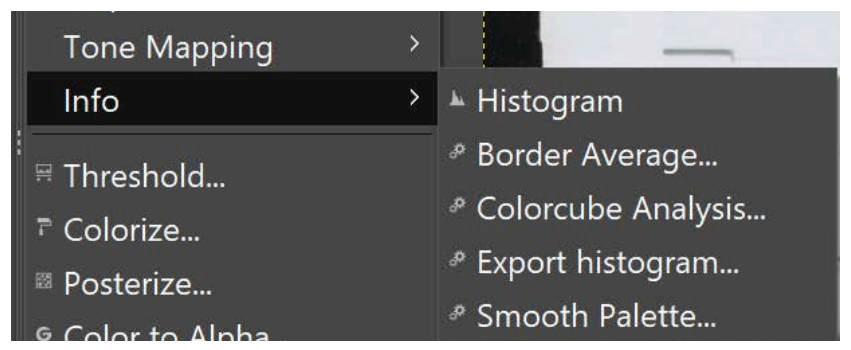

22. In the upper right area of the Gimp interface, you will now see a graph appear. This is called the HISTOGRAM. The Histogram plots out the tonal range of the image in a way that is easy to visually understand. A peak in the graph indicates a high concentration of the corresponding tone, shown in the narrow strip just below the graph.

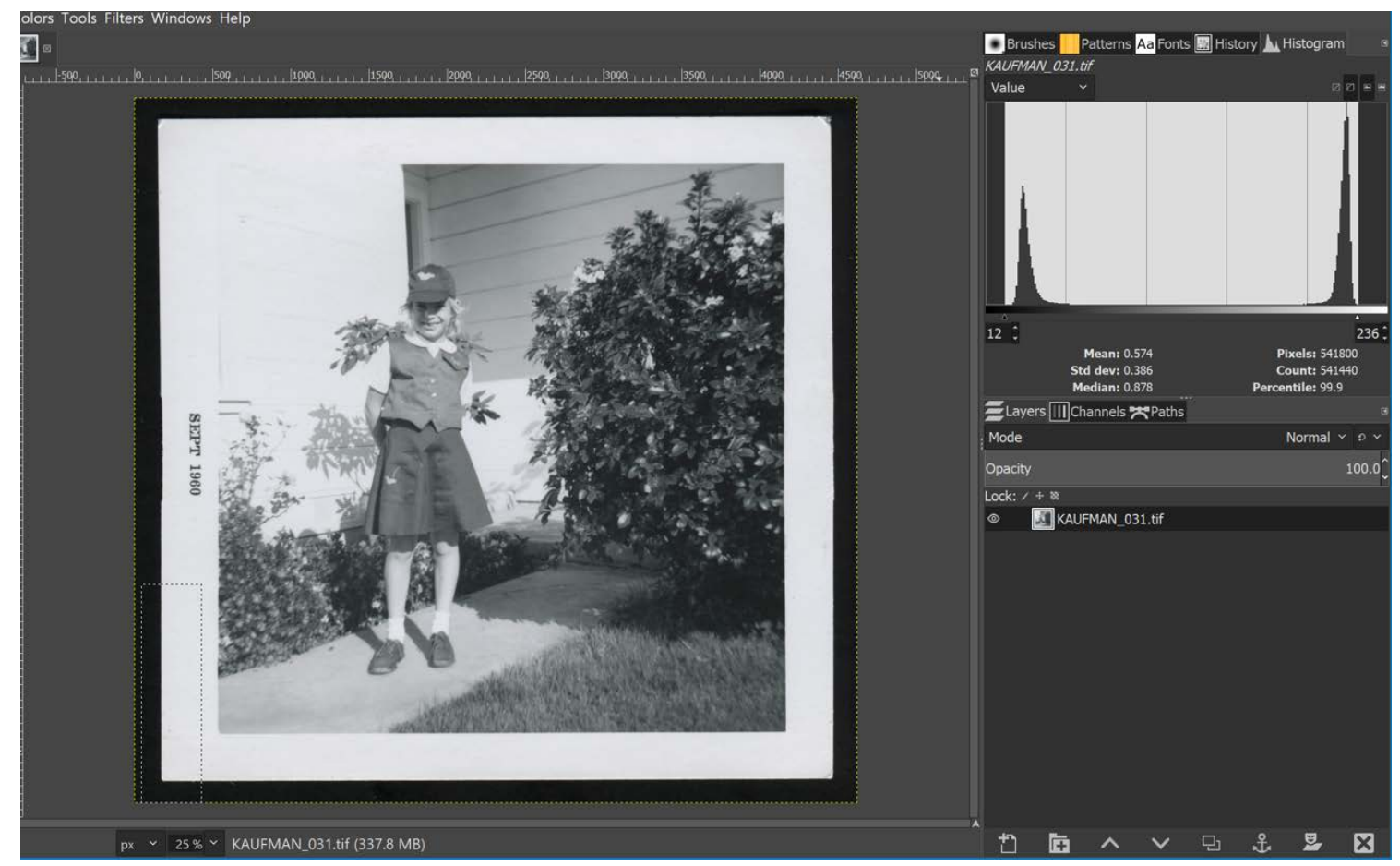

55. FADGI recommends that on a dark to light scale of 0 to 255 , highlights remain under 245 and shadows remain over 10 . 
23. Click on the point in the graph where the shadow peak meets the base of the graph, and then drag the cursor across the graph to the furthest right hand point where the highlight peak meets the base of the graph.
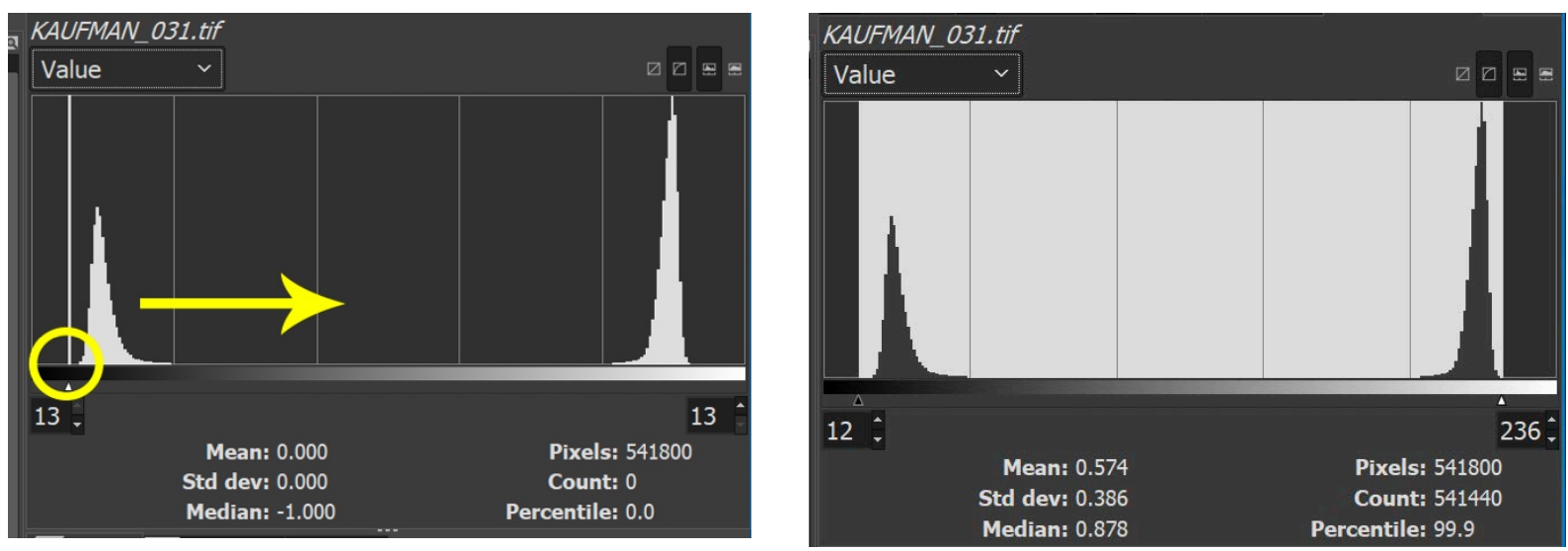

24. Now look at the numbers in the two boxes at either end of the histogram. If the numbers are between 10 and 245, then your scan is properly exposed and meets FADGI guidelines.

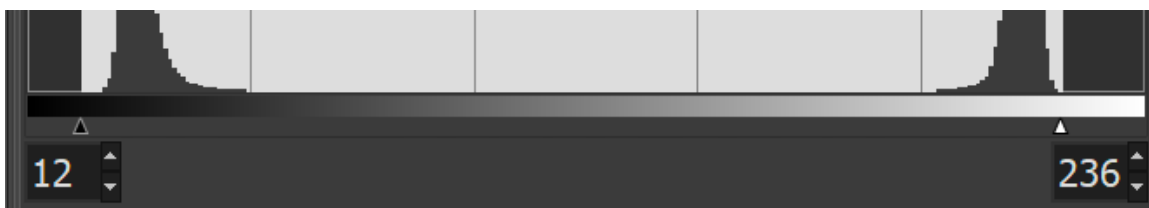

Keep in mind that the peaks represent the darkest area and the lightest area of the image, which in many cases is the black border you included in scanning and the white border in the photograph. As long as those two areas are between 10 and 245, you have a properly-exposed scan. Even with the values ranging from 10 to 245 , you may see that the photograph itself is either too dark, too light, too contrasty etc. This is simply because the photograph was not exposed properly when it was taken. When creative archival scans, you should not make adjustments to correct the exposure of the image itself on the MASTER / ORIGINAL scan. If you do want to correct the exposure, only do so on a PRODUCTION COPY of the original scan. 
25. To save the newly edited tiff as the original TIFF (which is allowed by FADGI guidelines) go to

\section{FILE > OVERWRITE}

26. To save the image as a different file type, go to FILE > EXPORT AS.

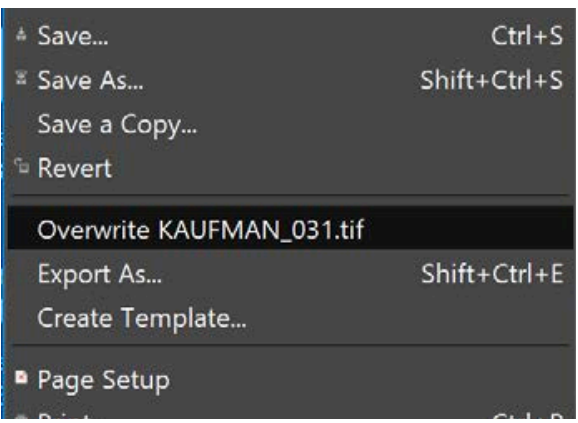

27. In the Export Image box that now appears, navigate to the folder to which you want to export the new file, and

Overwrite KAUFMAN_031.tif

Export As...

Shift+Ctrl+E

Create Template...

select the file type you want to save it as from the SELECT FILE TYPE dropdown menu. If you want to change or amend the file name you can do so in this step as well.

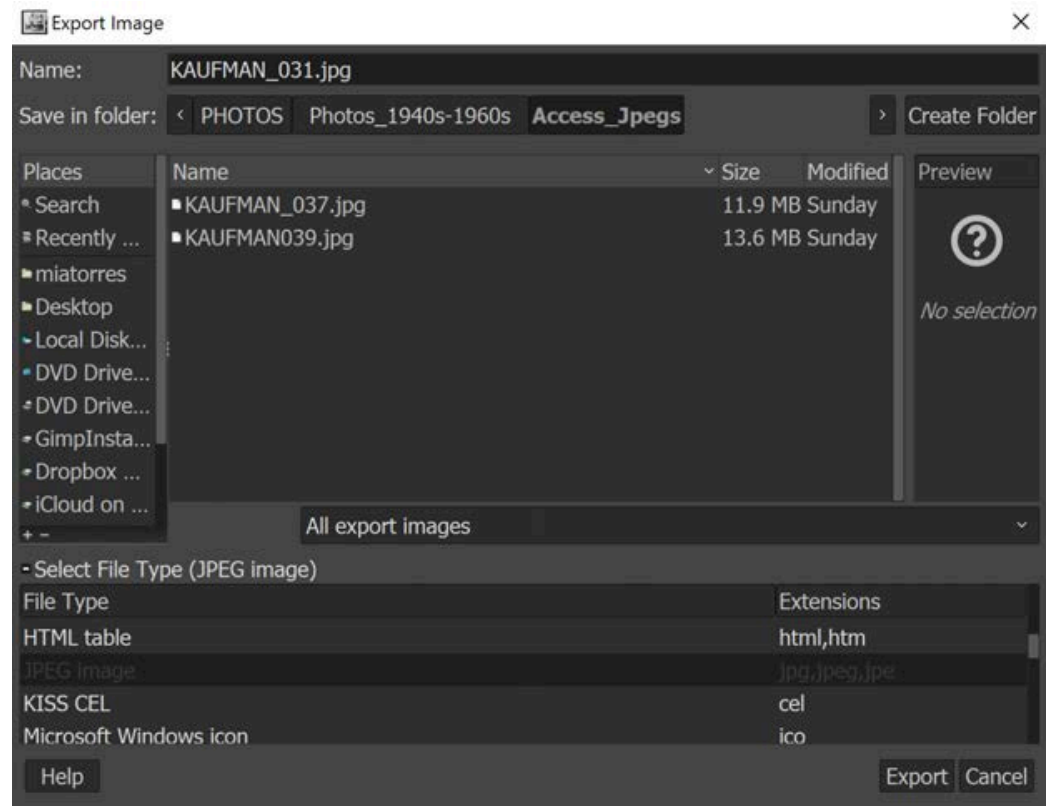

28. Depending on the file type you have chosen, a second export menu may appear. Here you can set your desired output quality. Here I am saving a JPEG with an output quality of 100 (percent).

29. Finally, click EXPORT.

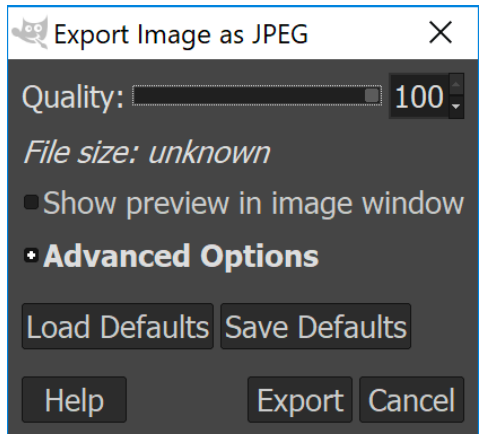




\subsection{Introduction to Metadata}

\section{What is Metadata?}

Simply put, metadata is information about information, or data about data. To further parse out the meaning of metadata, information scientist Jeffrey Pomerantz defines metadata as "a statement about a potentially informative object." ${ }^{56}$ The statements Pomerantz is referring to are the words, phrases, or numbers entered into predetermined groups of criteria, called schema. Metadata schema have different purposes intended to describe certain aspects of an object or record, and an object or record can be described simultaneously by multiple metadata schema.

Adding metadata to a digital file is like writing notes on the back of a photograph-who is in it, when it was taken and by whom, what the occasion was, etc. Metadata is information that is either generated when the file is created-the file name, date of creation, the file size, its dimensions, sometimes even the geographic coordinates at which it was taken and its copyright status. Like notes jotted on the back of a photograph, metadata travels everywhere with the document because it is embedded into the file and can be viewed and edited when the file is opened in certain programs.

\section{Types of Metadata}

According to the Getty Research Institute, all metadata describes either the content, context, or structure of a record, and can be categorized into five categories: administrative, descriptive, preservation, technical, and use. ${ }^{57}$ Administrative metadata describes the provenance or origin of the record, any copyright information there might be, appraisal information, etc. Descriptive metadata includes things like title, author, creator, date the original object was created, the subject, keywords, and descriptions.

56. Jeffrey Pomerantz, Metadata (Cambridge, MA: MIT Press, 2015), 26.

57. Anne J. Gilliland, "Setting the Stage," in Introduction to Metadata, edited by Murtha Baca, 3rd ed.

Los Angeles: Getty Publications, 2016, http://www.getty.edu/publications/intrometadata/setting-the-stage/ 
Preservation metadata, most often used by museums and archives, describes the condition of the record, if it has undergone any restoration or conservation, etc. Technical metadata is generated by the hardware used for digitization and provide information about the characteristics of the digital file, and use metadata, used most often by archives and libraries, describes circulation data, exhibition records, and copyright.

These types are divided groups of data fields, called schema, that have been created by different organization or industries to focus data into information sets that are relevant to that organization or field. For example, $\mathrm{DICOM}^{58}$ metadata encompasses information that is relevant to medicine and medical imaging, ISBN ${ }^{59}$ describes publication information, CSDGM $^{60}$ describes geographic information, EXIF ${ }^{61}$ metadata encompasses information that is automatically generated by cameras, scanners, and other digital recording devices, and IPTC metadata describes information that such the title of a file, the author or creator, a description, tags or keywords, location, event, etc. that is manually added by the user.

\section{Why is metadata important?}

Metadata is important because it provides information that allows for the categorization, analysis, and informative description of records. In the context of museums, libraries, and archives, metadata allows for extensive organization and quick recall of records that fit certain defined criteria. For example, instead of looking for a specific book by looking along each individual shelf on every single stack in the hopes of finding a book that may or may not be there in the first place, assigning sets of metadata to each book and then recording this information in a database (or card catalog if you want to get nostalgic), allows the searcher to very quickly search through every item in the library by recalling only those that match a specified set of criteria, like author, date, or subject.

58. Digital Imaging and Communications in Medicine

59. International Standard Book Number

60. Content Standard for Digital Geospatial Metadata

61. Exchangeable Image File 
Without the addition, inclusion, and compiling of metadata into a database, we would never be able to find anything, or quickly view information about a record or set of records.

After you have scanned items in your collection, the next step is to add metadata to the files. There are a number of different ways to do this depending on what operating system and what programs you are using. Metadata can be embedded into your files using a variety of programs, like Adobe Bridge, Photoshop, and Lightroom, Photo Mechanic, and Gimp. Some programs allow you to add more categories of information than others, but chances are if you are only adding metadata for your personal files (no model releases or licensing involved) you may only want to fill in a handful of fields. I find that it is easiest to edit metadata in both Bridge and Lightroom (the process is very similar), but there is also a variety of third party programs like Image Info Toolkit III and that allow you to edit and add metadata to your files.

Here are a few things to keep in mind when adding metadata to your files:

1 Metadata can be changed and edited. Embedded metadata is not set in stone, but will remain in the image unless deleted.

2 Do not remove or edit metadata from other people's files unless you have been given permission to do so.

3 Embedded metadata is removed from a file when it is uploaded to social media websites. $^{62}$

4 Metadata you have embedded into a file will remain embedded in all derivative files saves or exported from the original file (unless the metadata is removed) ${ }^{63}$

5 Embedding metadata to your files is not absolutely necessary. If you prefer to leave all of the information in a catalog or Excel spreadsheet, that is perfectly fine.

62. Yes, items 2 and 3 are in direct conflict with each other. Don't upload file to social media if you want to retain all copyright and permissions. Once the metadata has been stripped from a file, it is essentially impossible to track.

63. For example, metadata added to a TIFF in Lightroom will also be embedded in any files (such as a Jpeg or PNG version) derived from the original file. Because of this, it is best to add metadata before saving derivative file types. If you add metadata to a TIFF file, that metadata will not automatically be added to a JPEG file that was saved before you added the metadata to the TIFF. 


\subsection{Adding Metadata in Adobe Bridge}

Adobe Bridge is a digital asset management application that is part of the larger Adobe Creative Cloud program suite. Bridge allows to the viewing, organizing, renaming, and reformatting of files on your computer. It is also a very easy way to add metadata to your digitized files.

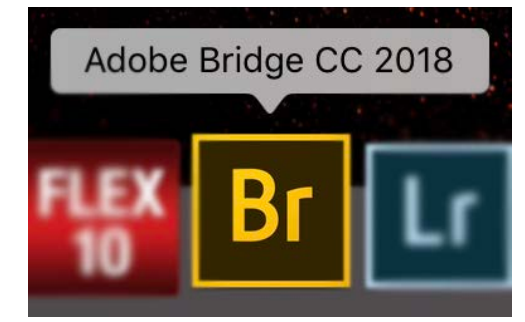

1. If needed, download the Bridge program from Adobe's website.

2. Open Bridge.

3. In the left side of the interface, select the FOLDERS tab and navigate to the folder of images to which you would like to add metadata. Note that no files are actually contained in Bridge. It is simply a way to view all of the files on your computer in a single interface.

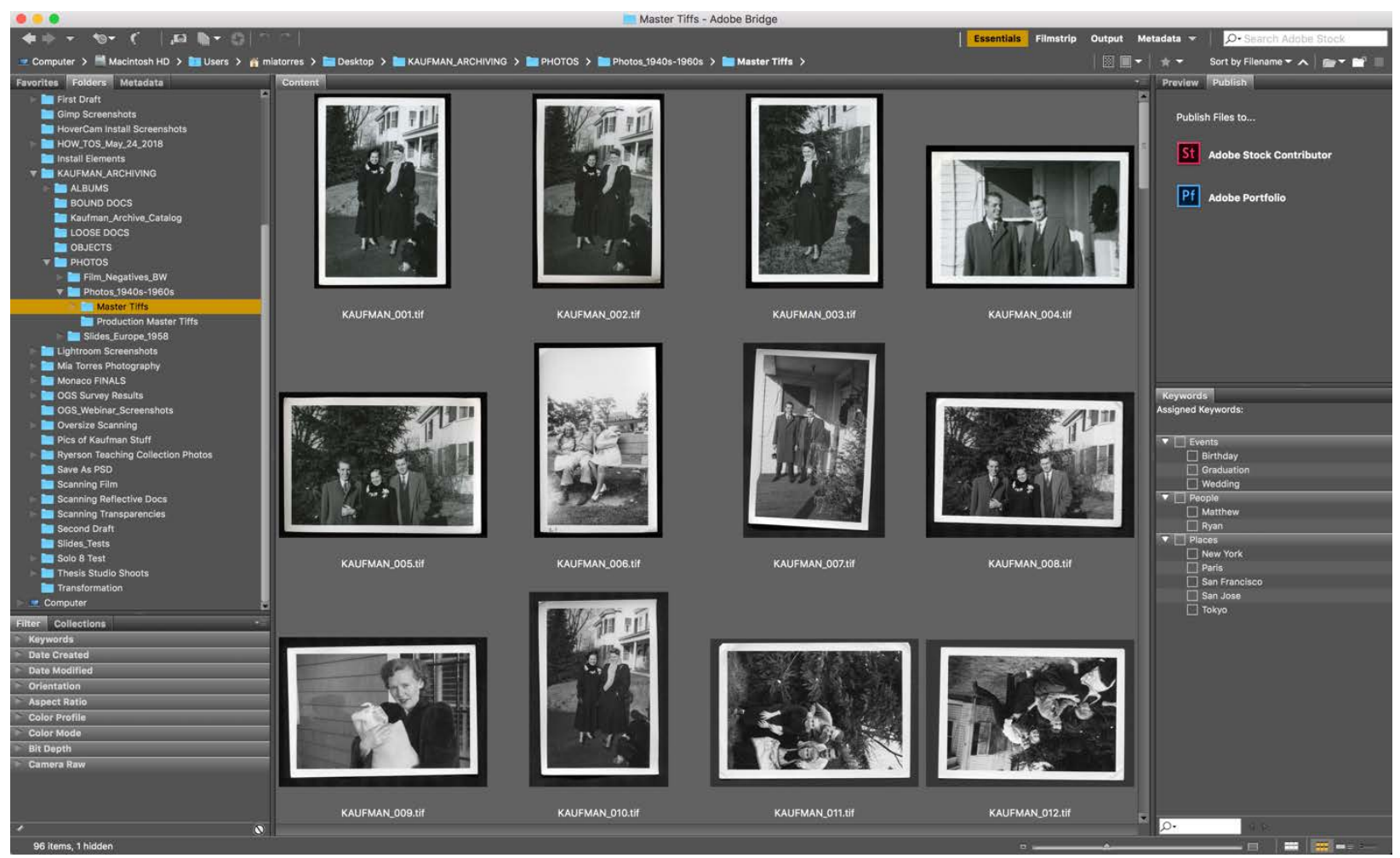


4. Select a photograph in the center pane. In the lower right corner of the program interface you can change the way the images are displayed (thumbnail, list, detailed list, etc.) by clicking on the different icons.

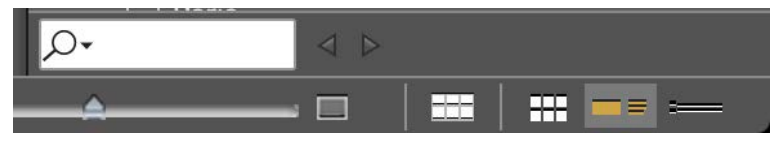

5. Click the METADATA tab on the left. Here you will see the File Properties (name, date taken, size, dimensions, resolution, bit depth, etc.), IPTC Core (creator, description, date, location, keywords, etc.), IPTC Extension (person shown, event name, location shown, model age, etc.), Camera Data, GPS, and others. Fill in any fields you feel are relevant and for which you have

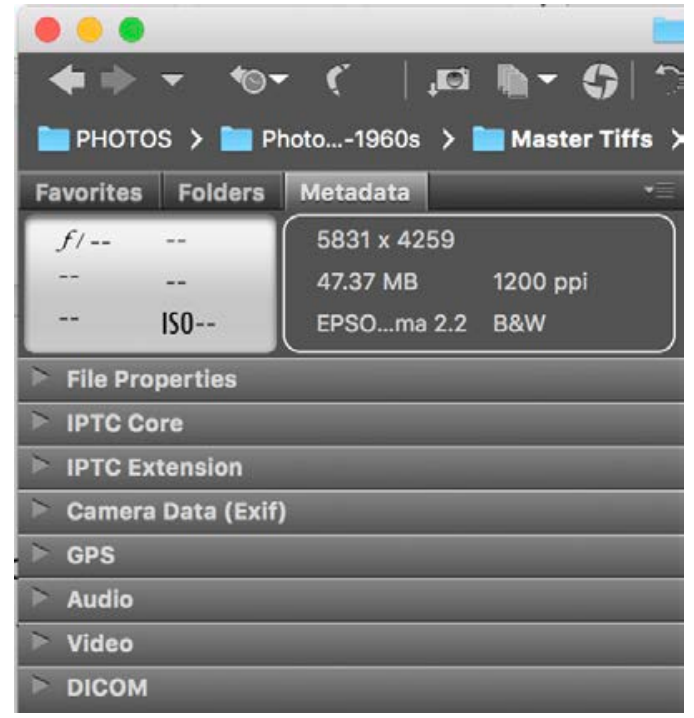
information.

6. When you click on another image, Bridge will ask you if you want to apply the metadata changes. Click APPLY.

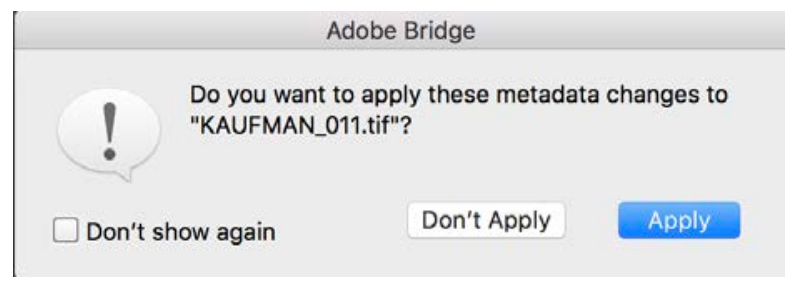

7. To add metadata to a group of images, select all the applicable images, fill in the metadata in the same way you would for a single image, and then click APPLY when prompted. 


\subsection{Adding Metadata on a Mac}

It is possible to add metadata to your image files directly through your computer's operating system. However, the metadata fields you can add are very limited-IPTC Core and Extended metadata fields that are available in Bridge and Lightroom are more comprehensive, but adding basic keywords and descriptions through the operating system can still be very helpful.

1. To add basic keywords and descriptions to an image file directly through the Mac operating system, click on the file you want to edit.

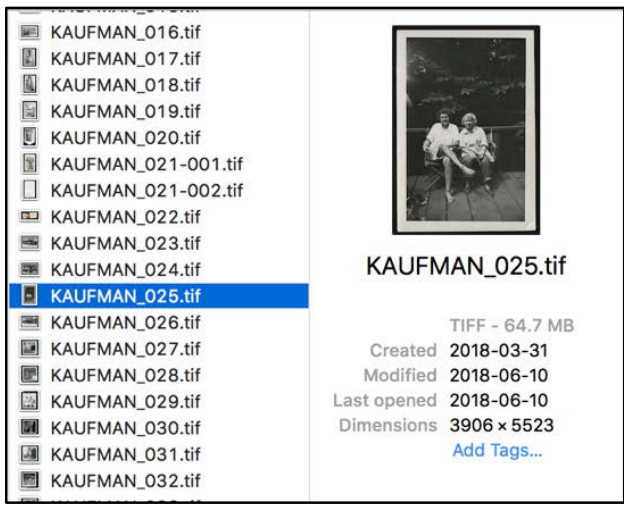

2. Next, go to FILE $>$ GET INFO or press COMMAND $+\mathbf{i}$.

3. In the text box just below the file name, type in any tags or keywords you feel are relevant. Type a comma or press ENTER between each one to indicate that they are separate keywords. Keywords that you have previously used will appear as suggestions while you type.

\begin{tabular}{|l|}
\hline KAUFMAN_025.tif \\
Modified: June 10, 2018 at 1:45 PM \\
\hline Conron 1950s \\
\hline Purple \\
Family Photography \\
Black and White \\
Kathryn Kaufman \\
Kaufman \\
Red \\
Show All... \\
\hline
\end{tabular}

- General:

Dore Info:

$\checkmark$ Name \& Extension:

KAUFMAN_025.tif

Hide extension

$\checkmark$ Comments:

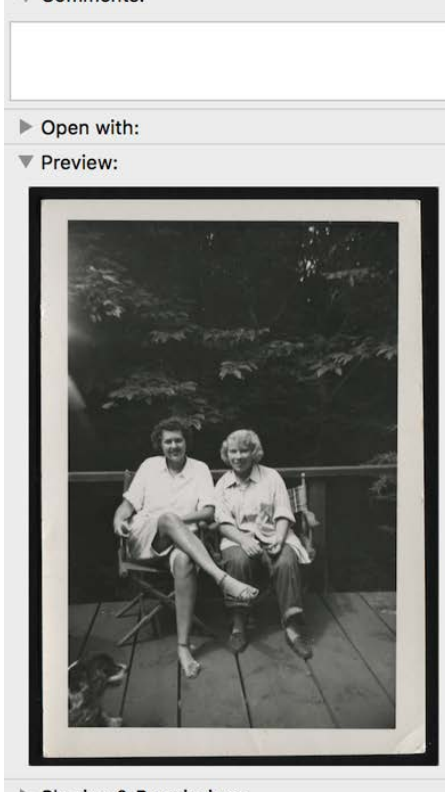


4. In the comments section, type a brief description of the photograph or any additional information that you want to make note of in the metadata. $\checkmark$ Comments:

Dorothy Conron and an unknown woman sit-in in folding chairs on a deck with a small dog. |

5. When you have finished adding in the tags and comments, click the RED $\mathbf{X}$ to close the window.

It is important to note that while you have added metadata to the file info, the metadata has not been embedded in the file itself, meaning that your tags and comments will not be readable by programs like Lightroom or Bridge. For the purposes of personal organization though, the tags are a great way to annotate and categorize your images. 


\subsection{Adding Metadata on a PC}

1. To add metadata on a PC, select the image you want to add information to.

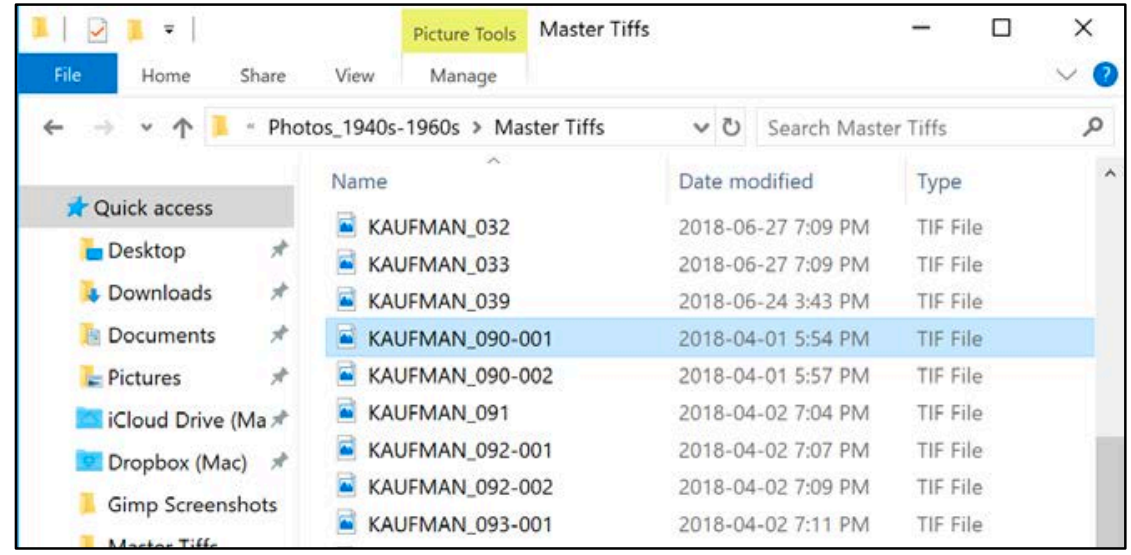

2. Next, go to HOME > PROPERTIES.

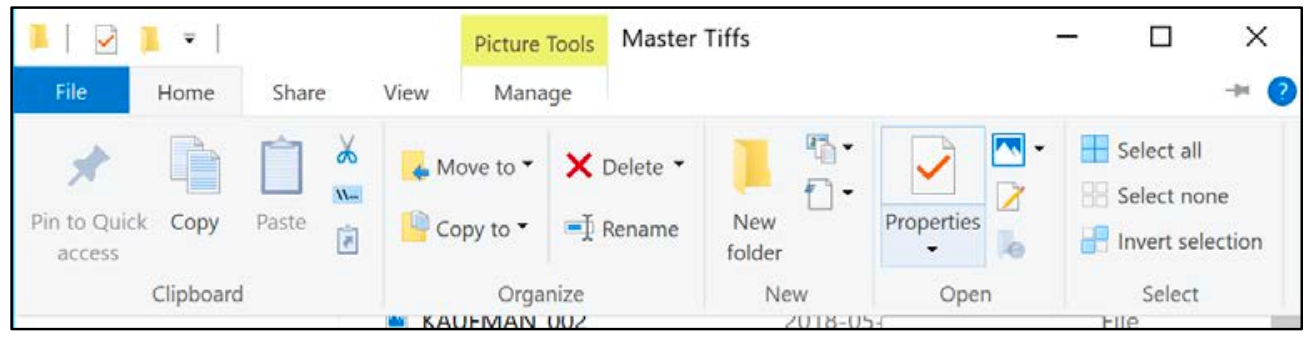

3. A window will appear with three tabs:

GENERAL, DETAILS, and PREVIOUS VERSIONS.

4. Go to the DETAILS tab.

\begin{tabular}{|c|c|c|}
\hline \multicolumn{3}{|c|}{ - KAUFMAN_090-001 Properties } \\
\hline General Details & Is Previous Versions & \\
\hline 田 & KAUFMAN_090-001 & \\
\hline Type of file: & TIF File (.tif) & \\
\hline Opens with: & Photos & Change... \\
\hline Location: & IMac|Home\Desktop|KAUFMAN & V_ARCHIVINGIPHOTC \\
\hline Size: & $47.6 \mathrm{MB}(49,951,242$ bytes $)$ & \\
\hline Size on disk: & $47.6 \mathrm{MB}(49,954,816$ bytes $)$ & \\
\hline Created: & April 1, 2018, 5:54:22 PM & \\
\hline Modified: & April 1, 2018, 5:54:22 PM & \\
\hline Accessed: & June 27, 2018, 9:12:16 PM & \\
\hline Attributes: & $\square$ Read-only $\square$ Hidden & $\square$ Archive \\
\hline & Cancel & Apply \\
\hline
\end{tabular}


5. In the Details tab you will see metadata fields like Title, Subject, Date, Comments, Copyright, etc. Fill in any and all information you have and feel is relevant. You may need to open the image in a window alongside of the Image Properties window to have a visual to reference.

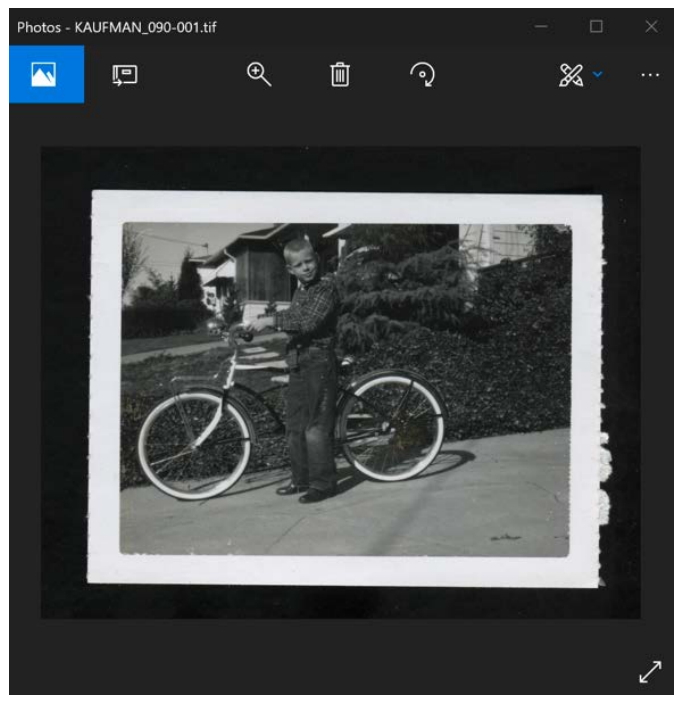

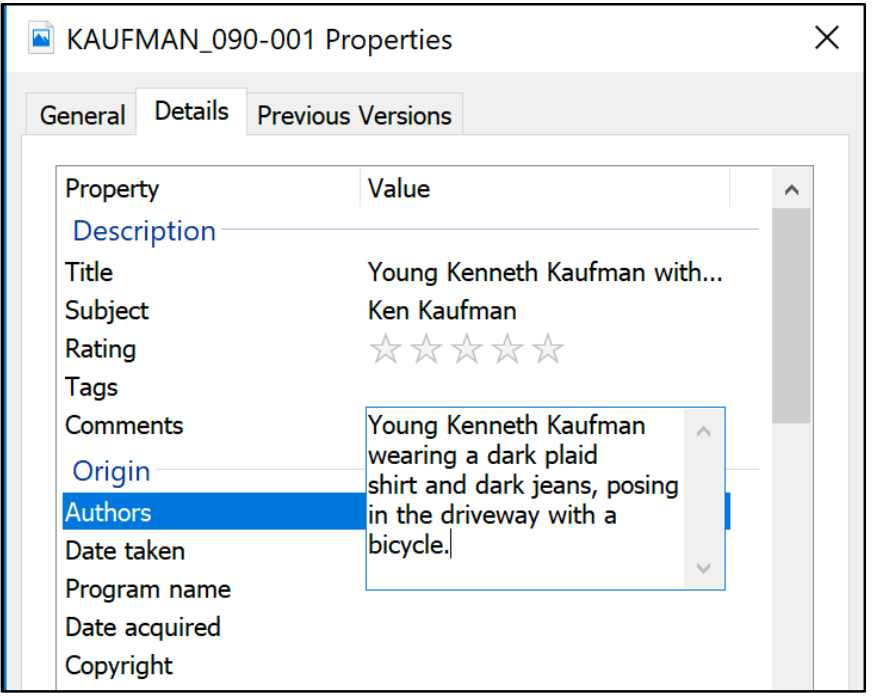

6. When you have finished adding information, click APPLY.

The information added to the image through the Properties window will only be visible when working with your files on the computer, through the same operating system. It will not be embedded in the file itself or readable by other programs such as Lightroom or Bridge. 


\section{SECTION 4: APPENDICES}




\subsection{FADGI Recommendations}

The Federal Administration Digitization Guidelines Initiative is an American governmental project that is focused on standardizing archival digitization using methods and techniques that ensure accurate and consistent results that they deem to be the most viable for long-term digital preservation. The guidelines provide information regarding records assessment, workflow, digitization environment, equipment, post production, and digital preservation. Numerous articles, reports, and other resources can be downloaded from their website: http://www. digitizationguidelines.gov/resources/

Here are the basic guidelines for digitizing most materials covered in this manual:

\section{DOCUMENTS (Bound)}

Master File: TIFF

Access Copy: Any format

DPI: 400 dpi minimum

Per Channel Bit Depth: 16-Bit

Colour Space: Colour (AdobeRGB 1998, ProPhoto)

\section{DOCUMENTS (Unbound)}

Master File: TIFF

Access Copy: Any format

DPI: 400 dpi minimum

Per Channel Bit Depth: 16-Bit

Colour Space: Colour (AdobeRGB 1998, ProPhoto)

\section{OVERSIZED MATERIALS}

Master File: TIFF

Access Copy: Any format

DPI: 400 or 800 dpi for detailed materials

Per Channel Bit Depth: 8 or 16-Bit

Colour Space: Colour or Grayscale (sRGB, AdobeRGB 1998, ProPhoto) 


\section{NEWSPAPERS}

Master File: TIFF

Access Copy: Any format

DPI: 400 dpi

Per Channel Bit Depth: 8-Bit

Colour Space: Colour or Grayscale (sRGB)

PRINTS AND PHOTOGRAPHS Graphic Arts prints, engravings, lithographs, drawings, watercolours, etc.

Master File: TIFF

Access Copy: Any format

DPI: 600 dpi minimum

Per Channel Bit Depth: 16 or 24-Bit colour

Colour Space: Colour (AdobeRGB 1998, ProPhoto)

PHOTOGRAPHIC TRANSPARENCIES (SLIDES) $35 \mathrm{~mm}, 120 \mathrm{~mm}, 4 \times 5$ formats

Master File: TIFF

Access Copy: Any format

DPI: 4000 dpi minimum

Per Channel Bit Depth: 16 color or grayscale (48-bit colour)

Colour Space: Colour (AdobeRGB 1998, ProPhoto)

Highlight Density: 245 max +/- 2

Shadow Density: $10 \mathrm{~min}+/-2$

PHOTOGRAPHIC TRANSPARENCIES (SLIDES) Larger than $4 \times 5$

Master File: TIFF

Access Copy: Any format

DPI: 2000 ppi minimum

Per Channel Bit Depth: 16 colour or grayscale

Colour Space: Colour (AdobeRGB 1998, ProPhoto)

Highlight Density: 245 max $+/-2$

Shadow Density: 10 min +/- 2

PHOTOGRAPHIC NEGATIVES $35 \mathrm{~mm}, 120 \mathrm{~mm}, 4 \times 5$ formats

Master File: TIFF

Access Copy: Any format 
DPI: 4000 dpi minimum

Per Channel Bit Depth: 16 colour or grayscale

Colour Space: Colour (AdobeRGB 1998, ProPhoto)

Highlight Density: 245 max $+/-2$

Shadow Density: 10 min +/- 2

PHOTOGRAPHIC NEGATIVES Larger than $4 \times 5$

Master File: TIFF

Access Copy: Any format

DPI: 2000 dpi minimum

Per Channel Bit Depth: 16 colour

Colour Space: Colour (AdobeRGB 1998, ProPhoto)

Highlight Density: 245 max +/- 2

Shadow Density: $10 \mathrm{~min}+/-2$

\section{PAINTINGS \& 2-D Art}

Master File: TIFF

Access Copy: Any format

DPI: 600 dpi minimum or 12,000 pixels on the long end.

Per Channel Bit Depth: 16 colour or grayscale

Colour Space: Colour (AdobeRGB 1998, ProPhoto)

Highlight Density: 245 max +/- 2

Shadow Density: $10 \mathrm{~min}+/-2$

\section{MICROFILM}

Monochrome $16 \mathrm{~mm}, 35 \mathrm{~mm}$, and black and white microfiche

Master File: TIFF

Access Copy: Any format

DPI: $4000 \mathrm{dpi}$

Per Channel Bit Depth: 8

Colour Space: Grayscale (Grey Gamma 2.2)

Highlight Density: 245 max $+/-5$

Shadow Density: 10 min +/- 5 


\subsection{Hard Drives}

Common External Hard Drives Based on 2018 Amazon.ca and B\&H prices (CDN)

\section{SATA DRIVES}

Drive:

Sizes:

Type:

Compatibility:

Price Range:

Drive:

Sizes:

Type:

Compatibility:

Price Range:

Drive:

Sizes:

Type:

Compatibility:

Price Range:

Drive:

Sizes:

Type:

Compatibility:

Price Range:

Drive:

Sizes:

Type:

Compatibility:

Price Range:
Western Digital My Passport External Hard Drive, USB 3.0 1TB, 2TB, 3TB, and 4TB

SATA

Mac and Windows

$\$ 55$ to $\$ 150$

Western Digital My Book Desktop External Hard Drive (Single Drive), USB 3.0

3TB, 4TB, 6TB, 8TB

SATA

Mac and Windows

$\$ 125$ to $\$ 250$

LaCie Rugged Mini USB 3.0 External Hard Drive 500GB, 1TB, 2TB, 4TB

SATA

Mac and Windows

$\$ 125$ to $\$ 225$

LaCie Rugged USB-C and USB 3.0 Portable Hard Drive 1TB, 2TB, 4TB, 5TB

SATA

Mac and Windows

$\$ 100$ to $\$ 240$

G-Technology G-DRIVE Mobile USB 3.0

1TB, 2TB, 3TB

SATA

Mac and Windows

$\$ 100$ to $\$ 185$ 
Drive:

Sizes:

Type:

Compatibility:

Price Range:
G-Technology G-DRIVE USB 3.0 External Hard Drive 2TB, 4TB, 6TB, 8TB, 10TB

SATA

Mac and Windows

$\$ 200$ to $\$ 450$

\section{SOLID STATE DRIVES}

Drive:

Sizes:

Type:

Compatibility:

Price Range:

Drive:

Sizes:

Type:

Compatibility:

Price Range:

Drive:

Sizes:

Type:

Compatibility:

Price Range:

Drive:

Sizes:

Type:

Compatibility:

Price Range:
Western Digital My Passport SSD, USB 3.1 256GB, 512GB, 1TB

Solid State Drive (SSD)

Mac and Windows

$\$ 135$ to $\$ 430$

Samsung T5 Portable SSD, USB 3.1 External SSD

250GB, 500GB, 1TB, 2TB

SSD

Mac and Windows

$\$ 130$ to $\$ 900$

SanDisk Extreme Portable SSD

250GB, 500GB, 1TB, 2TB

SSD

Mac and Windows

$\$ 135$ to $\$ 850$

Transcend USB 3.0 External Solid State Drive 128GB, 256GB, 512GB, 1TB

SSF

Mac and Windows

$\$ 100$ to $\$ 650$

\section{RAID HARD DRIVE SYSTEMS}

Note that you can only fill half of the total because RAID systems are comprised of 2 or more drives

Drive:

Western Digital My Book Desktop External Hard Drive (Dual Drive), USB 3.0 
Sizes:

Type:

Compatibility:

Price Range:

Drive:

Sizes:

Type:

Compatibility:

Price Range:

Drive:

Sizes:

Type:

Compatibility:

Price:

Drive:

Sizes:

Type:

Compatibility:

Price Range:
4TB, 6TB, 8TB, 12TB, 16TB, 20TB

SATA (RAID)

Mac and Windows

$\$ 425$ to $\$ 1,100$

LaCie Rugged Raid, Thunderbolt and USB 3.0 External Hard Drive

4TB

SATA (RAID)

Mac and Windows

$\$ 350$ to $\$ 450$

G-Technology G-RAID 2-Bay Thunderbolt 2 and USB 3.1

RAID Array

8TB, 12TB, 16TB, 20TB

SATA (RAID)

Mac and Windows

$\$ 1,400$

LaCie 2big Dock 2-Bay RAID Array Thunderbolt and USB 3.0 8TB, 12TB, 16TB, 20TB

SATA (RAID)

Mac and Windows

$\$ 800$ to $\$ 1,500$ 


\subsection{Colour Spaces}

Colour spaces are mathematical, often three-dimensional models used to visually express the range of colours a specific device or profile can show. Profiles are based in either 3 or 4 channels, Red, Green, and Blue (RGB), or Cyan, Magenta, Yellow, and Black $(\mathrm{CMYK})^{64}$. RGB is an additive colour space, one used to describe light waves, while CMYK is a subtractive colour space using reflected light, like ink on paper. This is why most printers use cyan, magenta, yellow, and black ink cartridges (and sometimes light versions of each as well) and computer screens emit light in an RGB colour space. The pixels that make up the device's screen has tiny light nodes, called sub-pixels, that can emit light in various patterns of red, green, and blue.

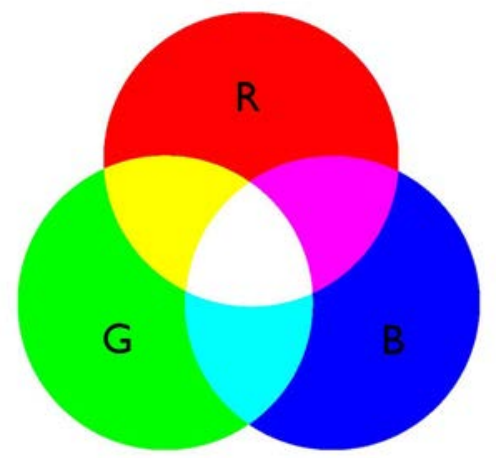

Additive Colour

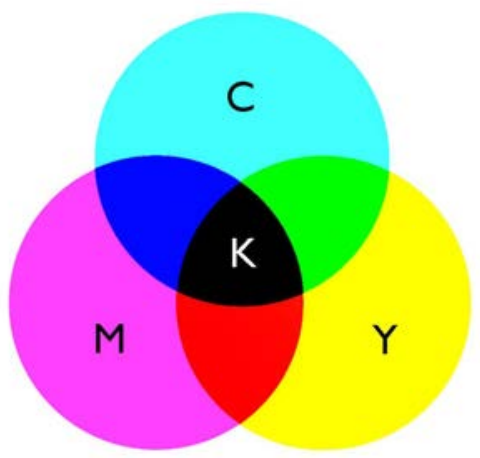

Subtractive Colour

Different colour spaces are able to express a different range of colours, some more than others. Common colour spaces used in digital imaging are sRGB, AdobeRGB 1998, and ProPhoto RGB.

64. Black is represented by the letter $\mathrm{K}$, a remnant of four-colour analogue printing in which the black colour was printed first as the key for the remaining three colour plates. 


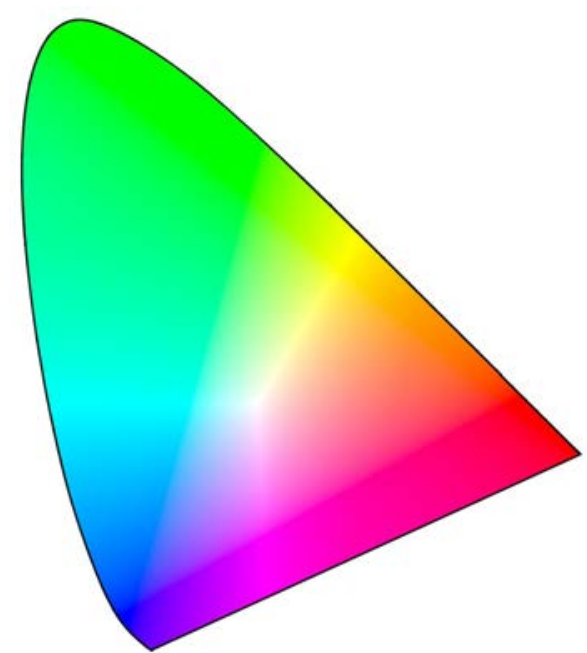

GIE I93I CHROMATICITY DIAGRAM

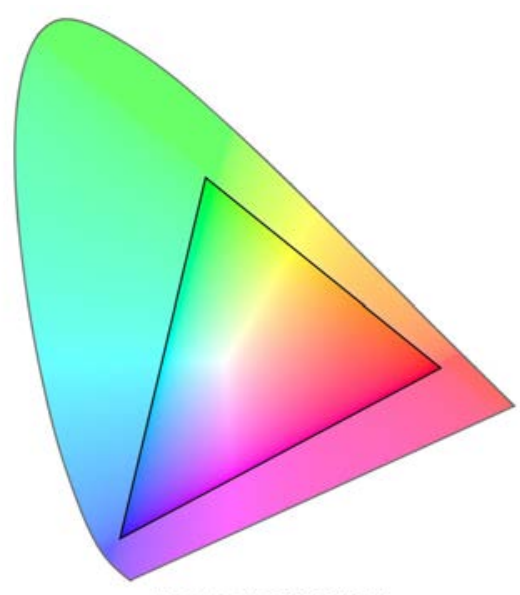

SRGB COLOUR SPACE

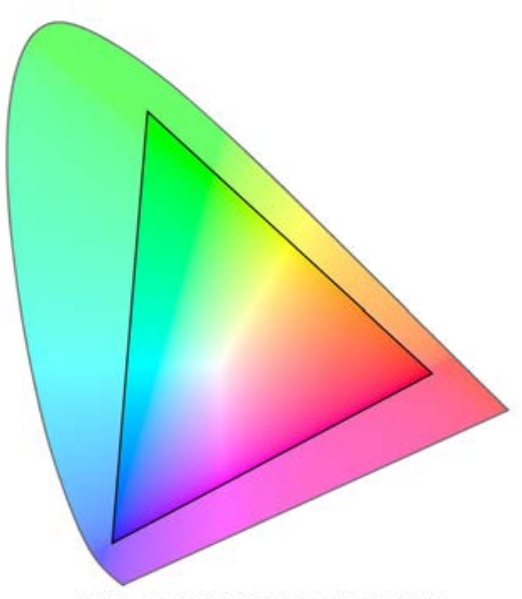

AdobeRGB 1998 COLOUR SPACE

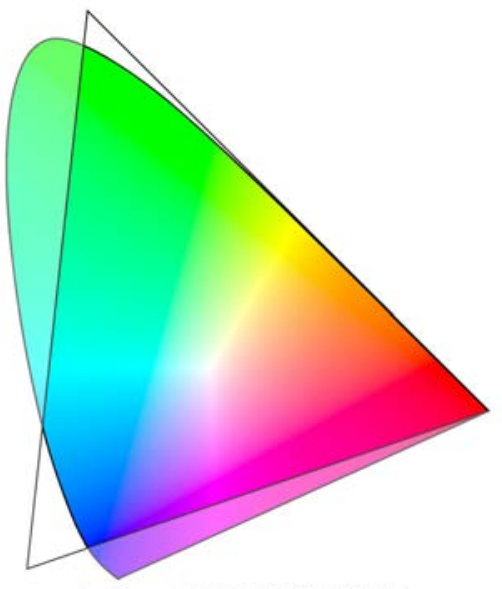

ProPhoto RGB COLOUR SPACE

Developed in 1931 by the Commission internationale de l'éclairage (International Commission on Illumination) ${ }^{65}$ the CIE 1931 Chromaticity diagram shown above is a two-dimensional representation of light visible to the human eye, encompassing all hues and saturation. Modern colour profiles are often laid over the CIE 1931 chromaticity diagram to illustrate how much and what areas of the visible colour spectrum the profiles are able to display. Here are the three main colour spaces briefly explained:

65. "Chromaticity Diagrams," Computer Graphics at Stanford University, https://graphics.stanford.edu/courses/cs178-10/applets/threedgamut.html (Accessed May 25, 2018).

205 


\section{SRGB}

Standard Red, Green, and Blue is a colour profile developed jointly by Microsoft and Hewlett-Packard in $1996^{66}$ as a standard colour space that could standardize the display of colours from the internet viewed on $\mathrm{CRT}^{67}$ monitors, which were the most common at the time. It is the smallest of the three colour spaces shown above.

\section{Adobe RGB (1998)}

Adobe RGB $(1998)^{68}$ is a colour profile developed and implemented by Adobe in 1998. Designed to be a versatile colour space, it is designed to translate well from screen to print and across many types of devices. Because Adobe products are staples of digital imaging and design, Adobe RGB (1998) is widely used.

\section{ProPhoto RGB}

Developed by Kodak in the early $2010 s^{69}$, the ProPhoto RGB colour space is designed to translate colours captured by a digital camera onto screen and into print as accurately as possible, minimizing colour loss ${ }^{70}$.

\section{So why does colour space matter?}

When you are scanning a document, you want to capture as much colour information as possible in that one scan. Some items may be too delicate to scan, physical handling is one of the major cause of damage to archival materials. Often in archives and museums records are cleaned, digitized, and rehoused all in one swoop so they will not need to

66. "History of the Very Odd SRGB Color Space," Nine Degrees Below Photography. https://ninedegreesbelow.com/photography/srgb-history.html (Accessed May 25, 2018).

67. Cathode ray tube displays, commercially produced since the 1920 s, were the predecessors to LCD (liquid crystal display) monitors which became popular in the early 2000s. I have no doubt that my inclusion of this explanation will amuse some older readers.

68. "Adobe RGB (1998) color image encoding," Adobe Digital Imaging Solutions, https://www.adobe.com/digitalimag/adobergb.html (Accessed May 25, 2018).

69. "2011 Color and Imaging Conference, Part VI: Special Session," RealTime Rendering, http://www.realtimerendering.com/blog/2011-color-and-imaging-conference-part-vi-special-session/ (Accessed May 25, 2018)

70. Jeff Schewe, The Digital Negative: Raw Image Processing in Lightroom, Camera Raw, and Photoshop (San Francisco: Peachpit Press, 2016). 
be handled again for a long time. The same principle should apply when digitizing your own records. Handle things as little as possible and when you do need to handle an item, do everything that needs to be done with that item (rehousing, scanning, etc.) at that time, to minimize any future need to handle it again.

When digitizing, you want to capture as much information as possible. This means scanning at a high resolution, as an archival file type (TIFF), and with as much colour detail information and fidelity as possible, which means using a large colour space, like ProPhoto RGB. Once you have this high quality "master" file, you can always save smaller versions as different file types, with different, smaller colour spaces. You can't accurately regenerate image data, so you always want to start with the most possible. 


\subsection{DPI Visual Reference Guide}

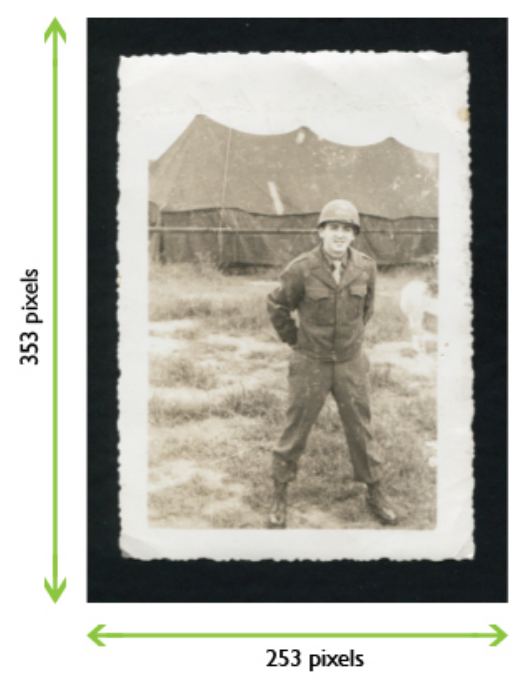

$72 \mathrm{dpi}$ (or pixels per inch) $253 \times 353$ pixels

24-bit color

277 Kilobytes (KB)

TIFF

USE: WEB and SCREEN
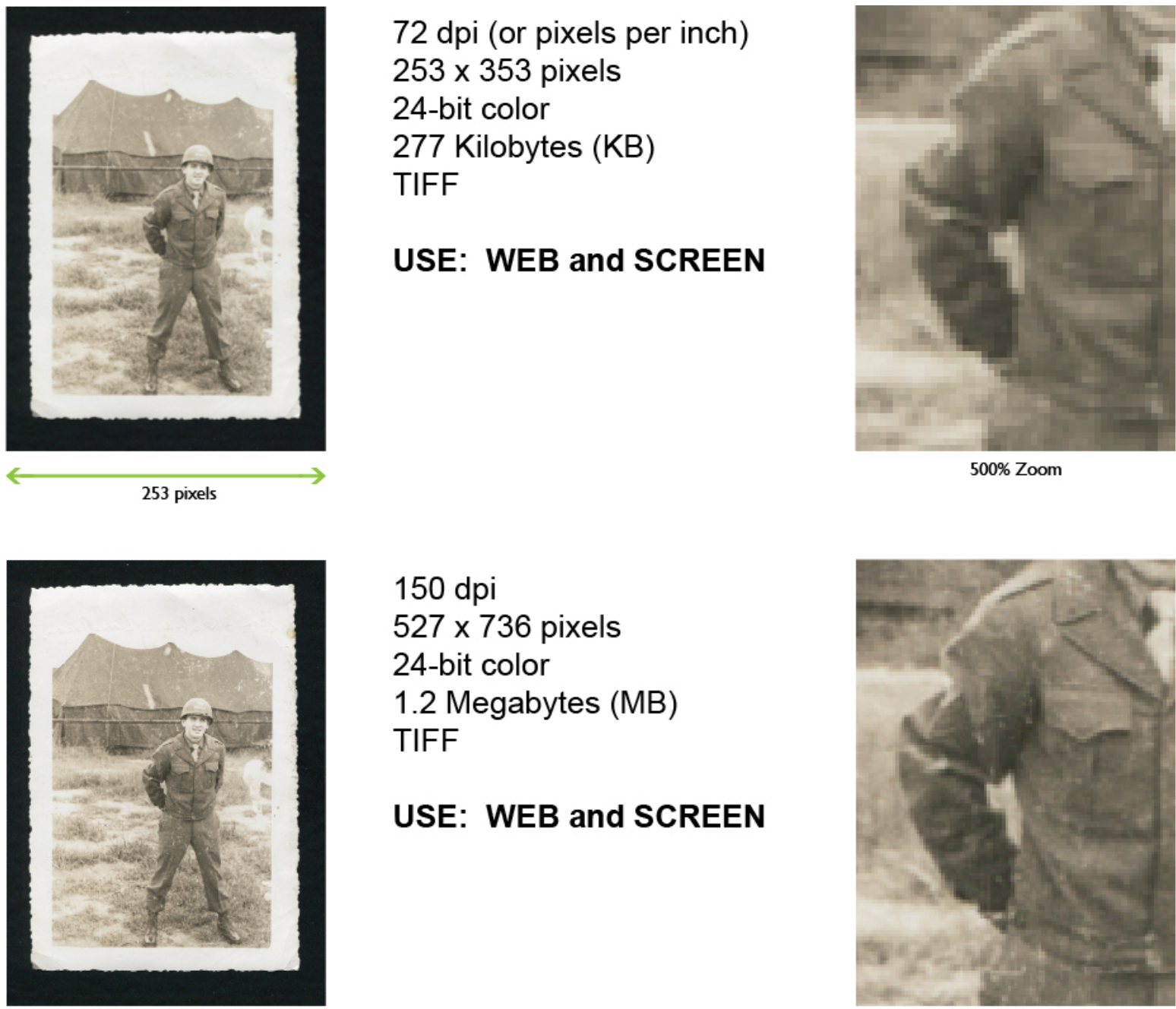

$150 \mathrm{dpi}$

$527 \times 736$ pixels

24-bit color

1.2 Megabytes (MB)

TIFF

USE: WEB and SCREEN

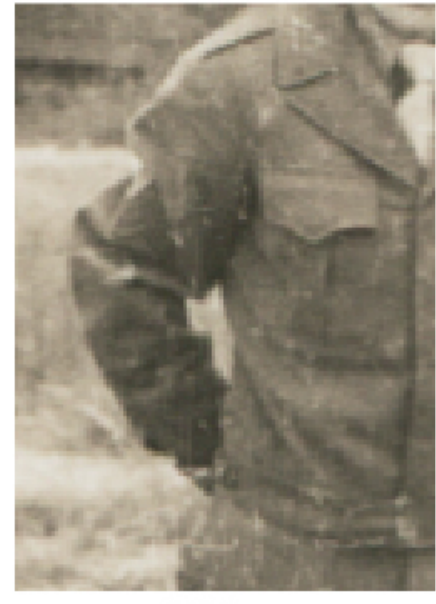

500\% Zoom

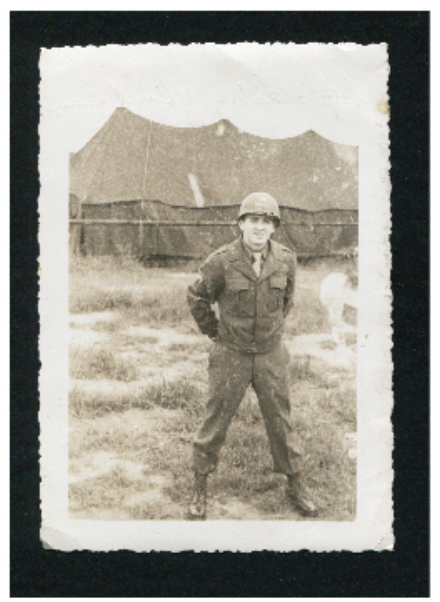

$300 \mathrm{dpi}$

1054 x 1472 pixels

24-bit color

4.7 MB

TIFF

USE: SMALL PRINT SIZES

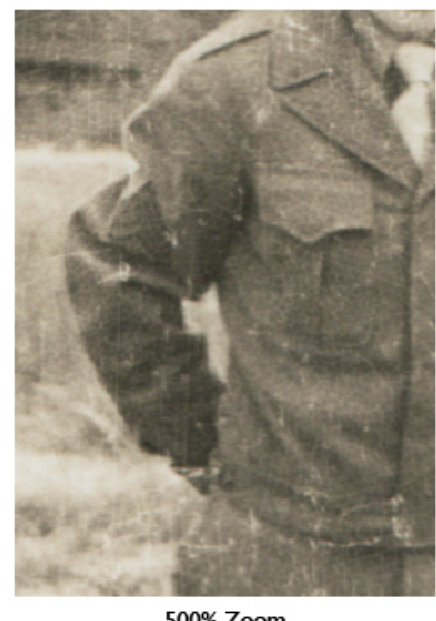

$500 \%$ Zoom 


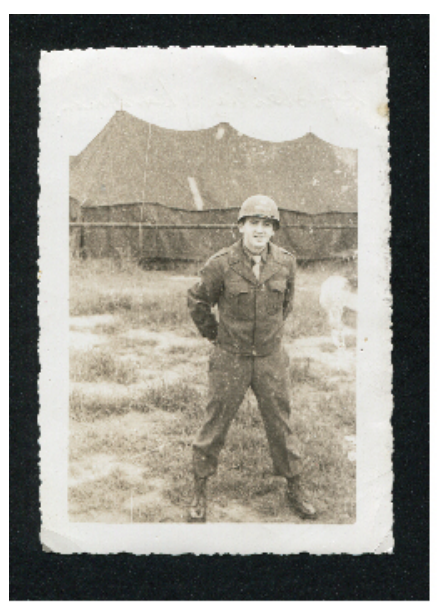

600 dpi

$2109 \times 2945$ pixels

24-bit color

$19 \mathrm{MB}$

TIFF

USE: MEDIUM PRINTS
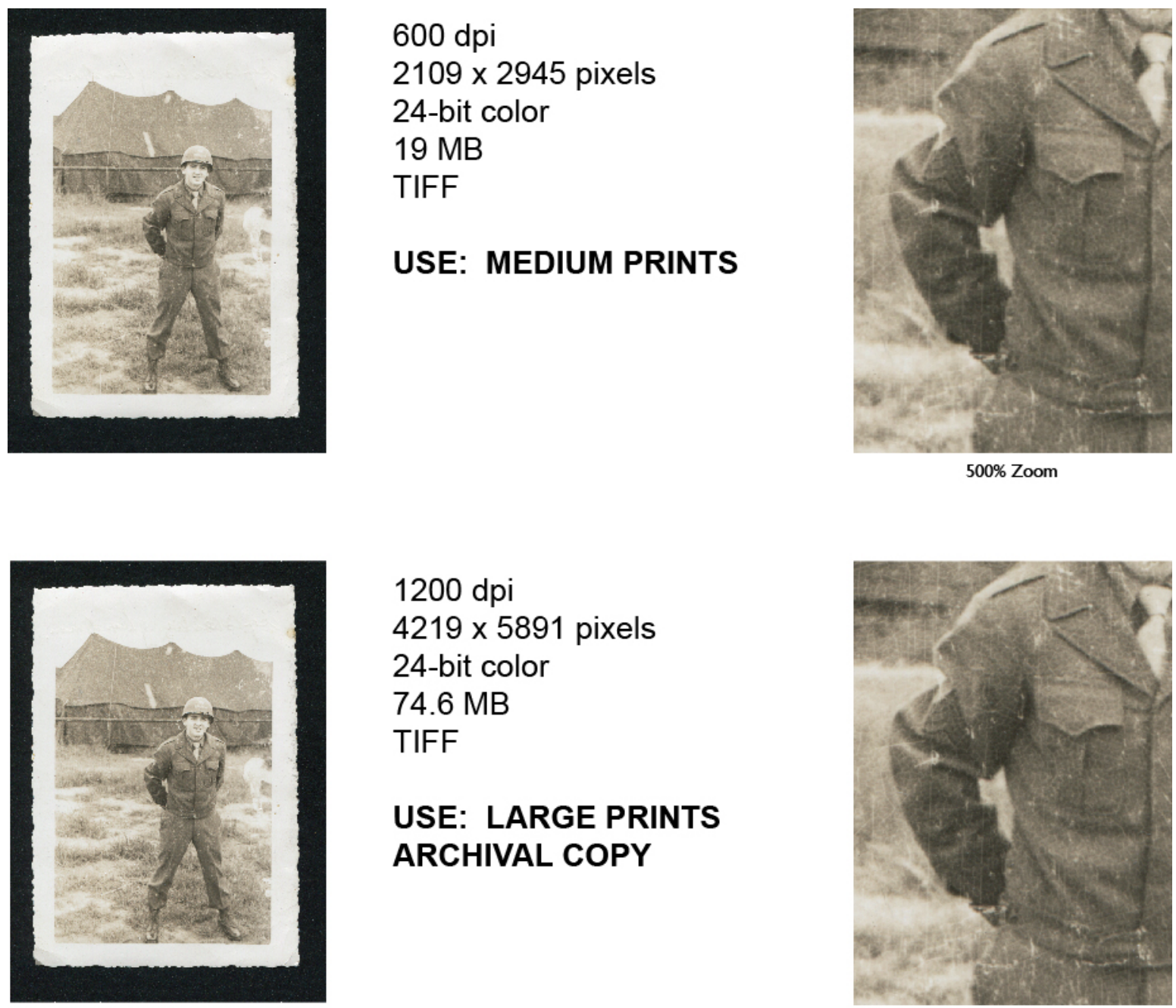

1200 dpi

4219 x 5891 pixels

24-bit color

74.6 MB

TIFF

USE: LARGE PRINTS

ARCHIVAL COPY

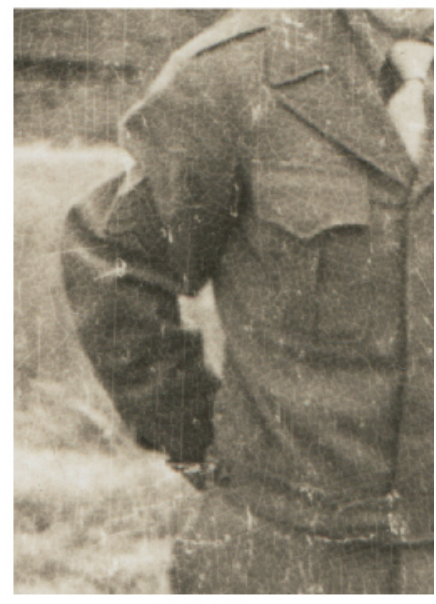

500\% Zoom

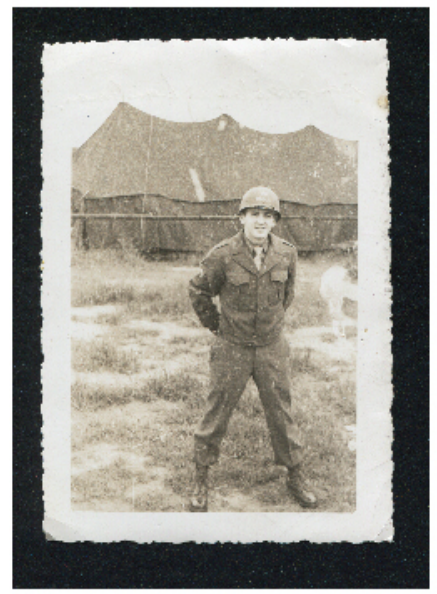

2400 dpi

8439 x 11782 pixels

24-bit color

298.3 MB

TIFF

USE: VERY LARGE PRINTS

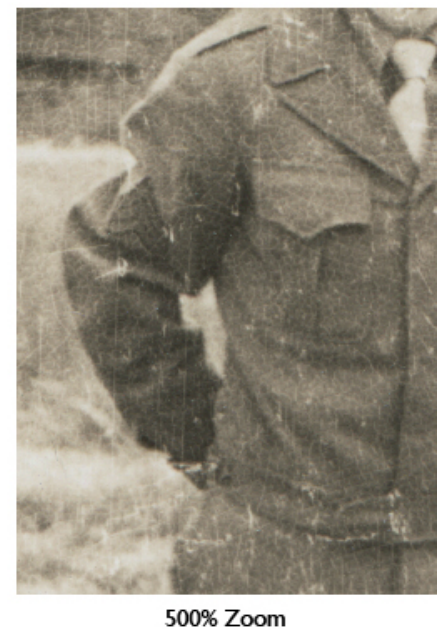




\subsection{HoverCam Solo 8Plus Quality Review}

Use of documents and images courtesy of the Ontario Genealogical Society.

\section{PHOTOGRAPHS}

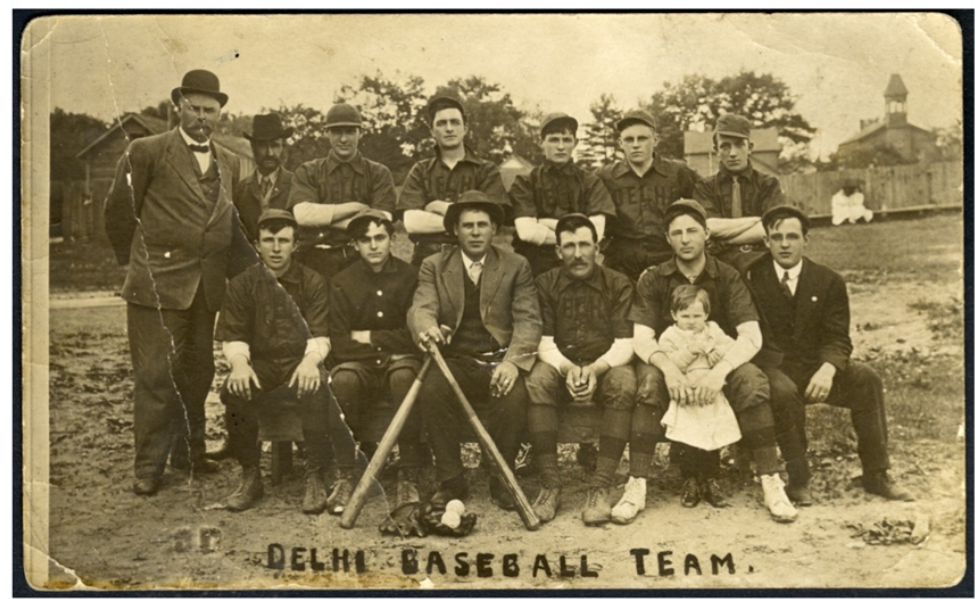

Postcard scanned with the Epson 11000XL flatbed scanner

- Physical Dimensions: $14 \times 8.5 \mathrm{~cm}\left(51 / 2 \times 3 \frac{3}{8}\right.$ in)

- Scan Dimensions: $4649 \times 3004$ pixels

- $25 \%, 100 \%$, and $300 \%$ zooms

- Scanning time: approx. 1 minute

- No colour or exposure adjustments
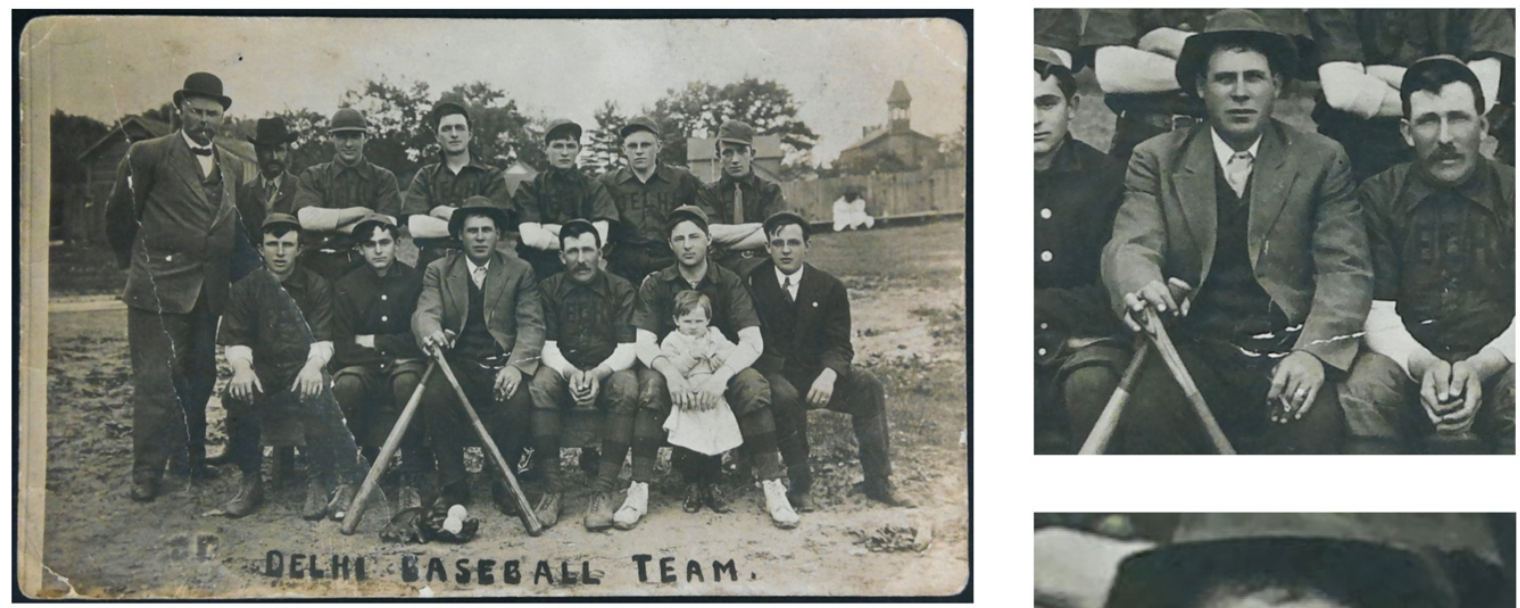

Postcard scanned with the HoverCam SOLO 8Plus document scanner

- Physical Dimensions: $14 \times 8.5 \mathrm{~cm}\left(51 / 2 \times 3 \frac{3}{8}\right.$ in)

- Scan Dimensions: $4952 \times 3108$ pixels

- $25 \%, 100 \%$, and $300 \%$ zooms

- Scanning time: approx 1 second (single click / exposure)

- No colour or exposure adjustments
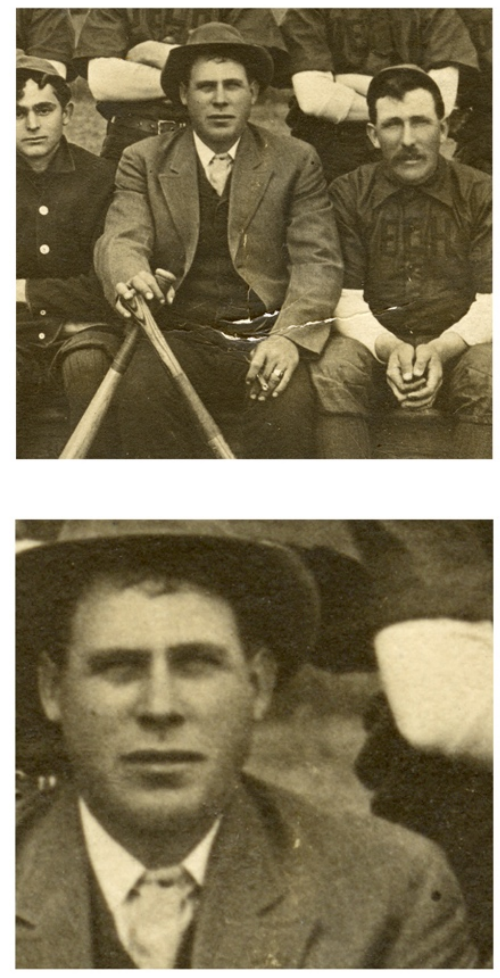

- Overhead fluourescent light source

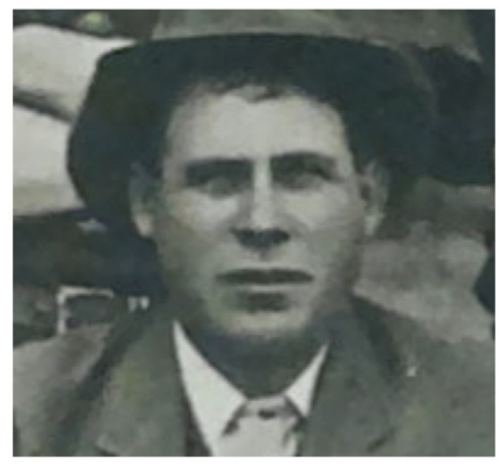




\section{DOCUMENTS}
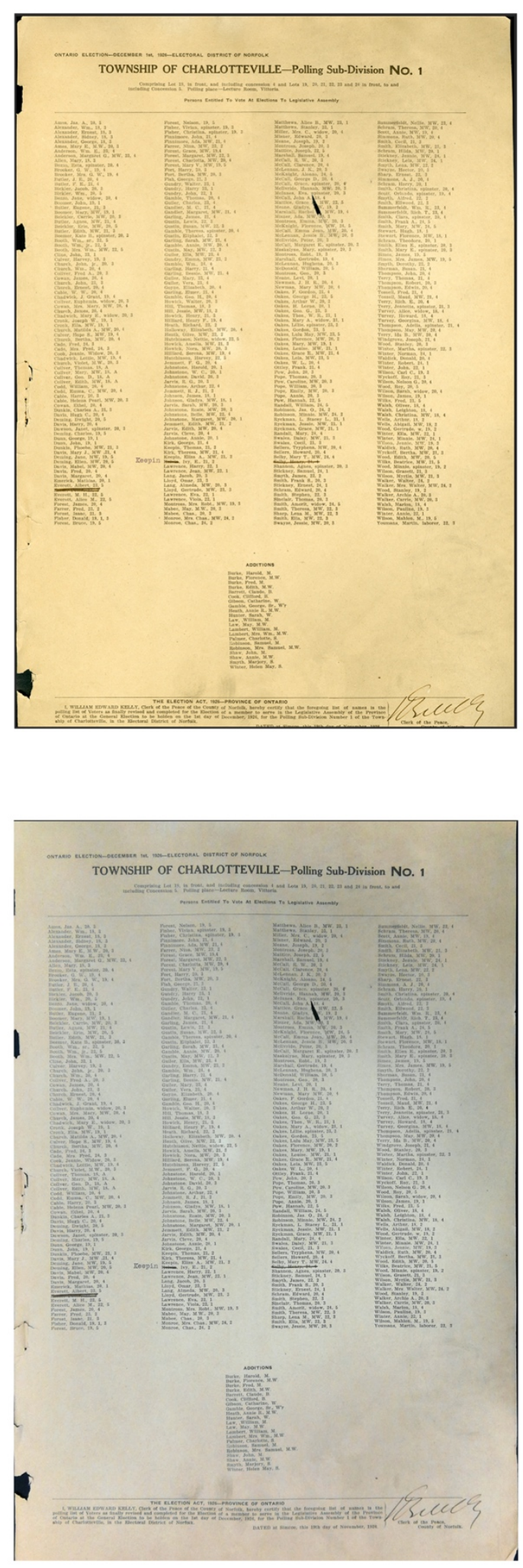

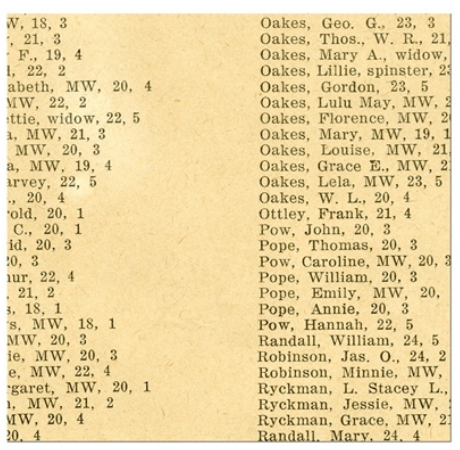

Oakes, Grace ., I Oakes, Lela, MW, Oakes, W. L., 20, Ottley, Frank, 21, Pow, John, 20, 3 Pope, Thomas, 20, Pow, Caroline, MW Pope, William, 20, Pope. Emily. MW

Voters' List scanned with the Epson 11000XL flatbed scanner

- Physical Dimensions: 46.4 x $30.5 \mathrm{~cm}$ (18 1/4 X 12 in)

- Scan Dimensions: $4291 \times 6132$ pixels

$-25 \%, 100 \%$, and $300 \%$ zooms

- Scanning time: approx. 3 minutes

- No colour or exposure adjustments

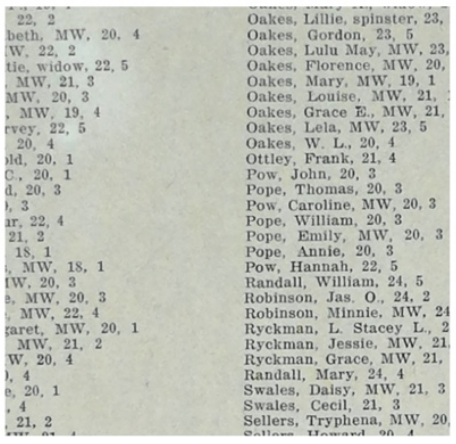

Oakes, Grace I., $\mathbf{N}$ Oakes, Lela, MW, Oakes, W. L., 20, Ottley, Frank, 21, Pow, John, 20, 3 Pope, Thomas, 20, Pow, Caroline, MW Pope, William, 20, Pope, Emily, MW

Voters' List scanned with the HoverCam SOLO 8Plus document scanner

- Physical Dimensions: 46.4 x $30.5 \mathrm{~cm}$ (18 1/4 X 12 in)

- Scan Dimensions: $4269 \times 6263$ pixels

- $25 \%, 100 \%$, and $300 \%$ zooms

- Scanning time: approx 1 second (single click / exposure)

- No colour or exposure adjustments

- Overhead fluourescent light source 


\section{HoverCam Solo 8Plus Quality Review: Conclusions}

\section{PROS:}

- Small, portable, light weight

- Easy-to-use interface

- Installation software included in the device (no internet connection needed)

- Can scan large documents

- Outputs a variety of file formats including lossless TIFFs (an "archival" file type)

- Outputs up to $210 \mathrm{MB}$ TIFFs with a single click

- Can scan large quantity of documents quickly (65-page legal-sized booklet in 6 minutes)

- Webcam and video capabilities, with audio

- Mac \& PC compatible

- Under \$500 CAD

\section{CONS:}

- Lower quality than most flatbed scanners (due to differences in types of optics used)

- Does not capture colours or detailed grain and tones well

- Difficult to scan shiny surfaces without reflection or glare (this can be compensated for by compositing in post-production.)

\section{USES:}

- Text-based or text-heavy documents that are too large to fit on the flatbed scanner

- Bound documents that cannot or should notbe unbound

- Do not use for materials that intended to be reproduced, blown up, printed, etc. for which colour accuracy is important.

\section{NOTES:}

The HoverCam Solo 8Plus would ideally be used in conjunction with a flatbed scanner. It is possible to make the HoverCam the solo method of digitization, but it should be noted that scanned photographs will be likely be adequate for research and representation purposes only, not for reproduction printing or enlargement. One goal of archival digitization is to generate digital files that are of high enough quality to create the highest quality print copies possible should the originals be lost or destroyed. However, due to financial and practical constraints, this cannot be achieved for every 
item scanned in every home. I believe finding a practical and affordable compromise between quality and quantity is understandable and justified. The HoverCam Solo 8Plus document scanner combines good quality with the capacity for high quantity output at an affordable price. 


\subsection{Digital Collections and Crowdsourcing Projects}

The following projects seek the help and knowledge of volunteers to transcribe and research archival documents in their collections.

\section{Africa Through A Lens}

http://www.nationalarchives.gov.uk/africal

Ancient Lives

https://www.ancientlives.org/ - /classify

\section{ArchHive}

http://transcribe.naa.gov.au/

Archives \& Access Project: AnnoTate

https://www.tate.org.uk/context-comment/blogs/archives-access-project-annotate-

crowdsourcing-transcribe-archives

Citizen Archivist Dashboard

https://www.archives.gov/citizen-archivist

Civil War Diaries and Letters

http://digital.lib.uiowa.edu/cwd/

\section{Clickworker}

https://www.clickworker.com

DIY History

http://diyhistory.lib.uiowa.edu/

Historic Newspaper Collection

https://cambridge.dlconsulting.com/

Louisville Leader

http://digital.library.louisville.edu/cdm/landingpage/collection/leader/

Map Warper

http://maps.nypl.org/warper/ 


\section{Metadata Games}

http://www.metadatagames.org/

\section{Project Gutenberg}

http://www.gutenberg.org/wiki/Gutenberg:Volunteering for Project Gutenberg

The Shakespeare Quartos Archive

http://www.quartos.org/

Transcribe Bentham

http://blogs.ucl.ac.uk/transcribe-bentham/

Trove

https://trove.nla.gov.au/

What's on the Menu?

http://menus.nypl.org/ 


\section{BIBLIOGRAPHY}

\section{LITERATURE REVIEW}

Albrecht Huber, Leslie. "Five Apps That Make Including Photos in Your Family Story a Cinch." FamilySearch Blog. May 10, 2017.

https://www.familysearch.org/blog/en/apps-including-photos-family-story-cinch/ (Accessed February 19, 2018).

Barratt, Nick. "From Memory to Digital Record: Personal heritage and archive use in the Twenty-first century," Records Management Journal, vol. 19, issue 1 (2009): 8-15. doi: 10.1108/09565690910937209.

Beidler, James M., "Roots \& Branches: Genealogy 'Leviathans' to Collaborate on Digitization," The Daily News, Sep. 8, 2013, http://ezproxy.lib.ryerson.ca/login?url=https://searchproquestcom.ezproxy.lib.ryerson.ca/docview/1430783745?accountid=13631 (Accessed Feb. 15, 2018).

Berger, Sherri, "The Evolving Ethics of Preservation: Redefining Practices and Responsibilities in the 21st Century," The Serials Librarian, vol. 57, no. 1-2 (July 20, 2009): 57-68. doi:10.1080/03615260802669086.

Bingham, Adrian, "The Digitization of Newspaper Archives: Opportunities and Challenges for Historians," Twentieth Century British History, vol. 21, no. 2 (2010): 225-31. doi:10.1093/tcbh/hwq007.

Bogus, lan, George Blood, Robin L. Dale, Robin Leech, and David Matthews. "Minimum Digitization Capture Recommendations." Association for Library Collections and Technical Services, 2013. http://www.ala.org/alcts/resources/preserv/minimumdigitization-capture-recommendations\#static media (Accessed Oct. 5, 2017).

Brown, Adrian. Practical Digital Preservation For Smaller Organizations. London: Facet Publishing, 2013.

Bülow, Anna E, and Jess Ahmon. Preparing Collections For Digitization. London: Facet Publishing, 2010.

Cameron, Fiona, and Sarah Kenderdine. Theorizing Digital Cultural Heritage: A Critical Discourse. Cambridge: The MIT Press, 2007.

Capell, Laura. "Method for Damaged Acetate Negatives: A Case Study Digitization Preservation." The American Archivist. vol. 73, no. 1 (2010): 235-49.

Capurro, Rafael. "Digitization as an ethical challenge." Al \& Society. vol. 32, issue 2 (May, 2017): 277-283. doi: 10.1007/s00146-016-0686-z. 
Cayley, Seth. "Digitization for the Masses: Taking Users Beyond Simple Searching in Nineteenth-Century Collections Online." Journal of Victorian Culture. vol. 22, no. 2 (2017): 1-8. http://doi.org/10.1080/13555502.2017.1301180.

Conway, Paul. "Preservation in the Age of Google: Digital Preservation, and Dilemmas." The Library Quarterly: Information, Community, Policy. vol. 80, no. 1 (January, 2010): pp. 61-79. http://doi.org/10.1086/648463.

Corrado, Edward M, and Heather Moulaison Sandy. Digital Preservation For Libraries, Archives, And Museums. 2nd ed. Lanham: Rowman \& Littlefield Publishers, 2017.

Coutts, Margaret. Stepping Away from the Silos: Strategic Collaboration in Digitisation. Oxford: Elsevier Science, 2016.

Coyle, Karen. "Mass Digitization of Books." The Journal of Academic Librarianship. vol. 32, no. 6 (2006): 641-45. https://doi.org/10.1016/j.acalib.2006.08.002

Digital Transitions: Division of Cultural Heritage. "Cultural Heritage Solutions Guide." 2016. https://dtdch.com/CulturalHeritage-SolutionGuide-2016-June-1.pdf (Accessed November 15, 2017).

Edwards, Mary Jane. "Some Darker Sides of Digitization; or, Disappearing Data, Doubtful Descriptions, and Other Deformations of Print." Style. vol. 49, no. 3 (2015): 321-333. http://doi.org/10.5325/style.49.3.0321

Ess, Charles. Digital Media Ethics. 2nd ed. Cambridge: Polity Press, 2014.

Frey, Franziska S., and James M. Reilly. 2006. Digital Imaging for Photographic Collections: Foundations for Technical Standards. 2nd ed. Rochester: Image Permanence Institute.

Government of Canada. "Capture Your Collections 2012 - Small Museum Version."

https://www.canada.ca/en/heritage-information-

network/services/digitization/capture-collections-small-museum.html\#05

(Accessed November 15, 2017).

Gray, Richard. "What a Vain Bunch We Really Are! 24 Billion Selfies Were Uploaded to Google Last Year." Daily Mail Online. June 01, 2016.

http://www.dailymail.co.uk/sciencetech/article-3619679/What-vain-bunch-really-24billion-selfies-uploaded-Google-year.html (Accessed March 22, 2018).

Guercio, M., \& Carloni, C. The research archives in the digital environment: The sapienza digital library project. JLIS. it, vol. 6, no. 1 (2015): 1-19. http://doi.org/10.4403/jlis.it-10989 
Harkema, Craig, and Cheryl Avery. "Milne En Masse : A Case Study in Digitizing Large Image Collections." New Review of Academic Librarianship 21 (2015): 249-55. http://doi.org/10.1080/13614533.2015.1034806

Higgins, Sarah. "Digital Curation: The Emergence of New Discipline." The International Journal of Digital Curation. vol. 6, no. 2 (2011): 78-88. http://doi.org/10.2218/ijdc.v6i2.191

Higgins, Sarah. "The DCC Curation Lifecycle Model." The International Journal of Digital Curation. vol. 3, no. 1 (2008): 134-140. http://doi.org/10.2218/ijdc.v3i1.48

Hudson LN, Blagoderov V, Heaton A, and Price BW et al. Holtzhausen P, Livermore L. "Inselect: Automating the Digitization of Natural History Collections." PLoS One. vol. 10, no. 11 (2015): 1-16. http://doi.org/10.1371/journal.pone.0143402

Klotz, Frieda. "Where Digitization is Failing to Deliver." MIT Sloan Management Review vol. 57, no. 3 (Spring, 2016). http://ezproxy.lib.ryerson.ca/login?url=https://searchproquest-com.ezproxy.lib.ryerson.ca/docview/1778453924?accountid=13631

Knight, Gareth. "The Forensic Curator: Digital Forensics as a Solution to Addressing the Curatorial Challenges Posed by Personal Digital Archives." The International Journal of Digital Curation. vol. 7, issue 2 (2012): 40-63. http://doi.org/10.2218/ijdc.v7i2.228

MacEntee, Thomas. Digitization Options For Family Photos Including Slides, Film Negatives, And Home Movies. Ebook. 1st ed. Hack Genealogy: 2015. https://ogs.on.ca/shop/digitization-options-family-photos-including-slides-filmnegatives-home-movies/

Murrell, Mary. "Out of Print: The Orphans of Mass Digitization." Current Anthropology. vol. 58, no. 15 (2017): 149-59. http://doi.org/10.1086/688868

Nash, Jeff, Sara Sterkenburg, and Paul Wentzell. "Project Plan for Digitization: A Structural Manual for Policy Development at the Greater West Bloomfield Historical Society." Written as a partial requirement of SI 644: Advanced Preservation. University of Michigan School of Information. 2011. https://www.gwbhs.org/documents/2012/11/si-644-digitization-project -plan.pdf (Accessed October 10, 2017)

Newell, Jenny. "Old Objects, New Media: Historical Collections, Digitization and Affect." Journal of Material Culture. vol. 17, no. 3 (2012): 287-306. http://doi.org/10.1177/1359183512453534

Northeast Document Conservation Center. "6.3 Digital Technology Made Simpler." NEDCC Preservation Leaflet. https://www.nedcc.org/free-resources/preservation- 
leaflets/6.-reformatting/6.3-digital-technology-made-simpler (Accessed November 15, 2017).

Northeast Document Conservation Center. "6.6 Preservation and Selection for Digitization." NEDCC Preservation Leaflet. https://www.nedcc.org/freeresources/preservation-leaflets/6.-reformatting/6.6-preservation-and-selection-fordigitization (Accessed November 15, 2017).

Pavlidis, George, Anestis Koutsoudis, Fotis Arnaoutoglou, and Vassilios Tsioukas. "Methods for 3D Digitization of Cultural Heritage." Journal of Cultural Heritage. no. 8 (2007): 93-98. http://doi.org/10.1016/j.culher.2006.10.007

Puglia, Steven, Jeffrey Reed, and Erin Rhodes. "Technical Guidelines for Digitizing Archival Materials for Electronic Access : Creation of Production Master Files." U.S. National Archives and Records Administration, Federal Agencies Digitization Initiative Still Image Working Group, (2010): 1-87. http://www.digitizationguidelines.gov/guidelines/FADGI Still ImageTech Guidelines 2010-08-24.pdf (Accessed November 15, 2017).

Sartori, Andrea, and Luciana Lazzeretti. "The Role of Digital Technologies in Heritage Interpretation: A Conceptual Framework and Some Recommendations," presented at 18th ICOMOS General Assembly and Scientific Symposium: Heritage and Landscape as Human Values, November 10-14, 2014, Florence, Italy. https://www.researchgate.net/publication/280089443 (Accessed November 15, 2017).

Sartori, Andrea. "Towards an intellectual history of digitization: Myths, dystopias, and discursive shifts in museum computing." Digital Scholarship in the Humanities. vol. 31, no. 2 (June, 2016): 428-440. http://doi.org/10.1093/llc/fqv006

Teper, Jennifer Hain and Emily Shaw. "Planning for Preservation During Mass Digitization Projects." Libraries and the Academy. vol. 11, no. 2 (2017): 717-39. http://doi.org/10.1353/pla.2011.0017

Viera, Al. "The Evolution of Digitization." Today. vol. 30, no. 2 (March, 2007): 16-18. http://ezproxy.lib.ryerson.ca/login?url=https://search-proquestcom.ezproxy.lib.ryerson.ca/docview/224483452?accountid=13631 (Accessed November 15, 2017).

Volpe, Andrea L. "Archival Meaning: Materiality, Digitization, And The NineteenthCentury Photograph." Afterimage Vol. 36, No. 6 (May, 2009): 11-16. http://ezproxy.lib.ryerson.ca/login?url=https://search-proquestcom.ezproxy.lib.ryerson.ca/docview/212090179?accountid=13631 (Accessed November 15, 2017). 
Wick, Harrison, and Rebecca Skirvin. "Managing Digital Archival Material on a Shoestring Budget Special Concerns in Micro-Shops." 2015.

http://www.midwestarchives.org/ccboard/27362969 266cc3f460b9b08276d96168c 5890cde.pdf (Accessed Oct. 14' 2017).

Williams, Don, and Louisa Jaggar. Saving Stuff: How to Care for and Preserve Your Collectibles, Heirlooms, and Other Prized Possessions. New York: Fireside Simon \& Schuster, 2005.

Woodley, Mary S. "Digital Project Planning \& Management Basics." Trainee Manual. Library of Congress: Cataloger's Learning Workshop. 2008. https://www.loc.gov/catworkshop/courses/digitalprojplan/pdf/Trainee-Final.pdf

Zhou, Yongli. "Fulfill Your Digital Preservation Goals with a Budget Studio." Information Technology and Libraries. vol. 35, no. 1 (March, 2016): 26-50. http://doi.org/10.6017/ital.v35i1.5704

"The Partial Digitization Of World History." New York Times, Late Edition (East Coast). March 18, 2007. http://ezproxy.lib.ryerson.ca/login?url=https://search.proquest.com/docview/43353 \%0D8659?accountid=13631

"Planning And Budgeting Digitization Projects". 2003. digital.library.ucla.edu. Accessed October 11, 2017. http://digital.library.ucla.edu/about/planning/budget.html.

"These 3 Smart Devices Let You Record Genealogy Documents, Photos and Stories Anywhere". Family History Daily. November 30, 2017. https://familyhistorydaily.com/genealogy-help-and-how-to/record-genealogydocuments-photos-anywhere/ (Accessed February 19, 2018).

"This May be the Fastest Way Ever to Scan Old Family Photos, and it's Free." Family History Daily. November 30, 2017. https://familyhistorydaily.com/genealogy-helpand-how-to/record-genealogy-documents-photos-anywhere/ (Accessed February 19, 2018).

\section{SECTION 1}

Edwards, Elizabeth and Janice Hart, ed. Photographs Objects Histories: On the Materiality of Images. London: Routledge, 2010.

Ericksen, Hilary and Ingrid Unger, ed. The Small Museums Cataloguing Manual: A Guide to Cataloguing Object and Image Collections. Victoria: Museums Australia, 2009.

Hirsch, Marianne, Family Frames: Photography, Narrative and Postmemory. Cambridge: Harvard University Press, 2016. 
Krogh, Peter. "Backups." The DAM Book: Digital Asset Management For Photography. http://thedambook.com/dyp/backups/ (Accessed June 13, 2018).

Langford, Martha M. and Staff, McCord Museum of Canadian Hisotry. Suspended Conversations: The Afterlife of Memory in Photographic Albums. Montreál: McGillQueens University Press, 2014.

Murtha, Baca. Cataloguing Cultural Objects: CCO; a Guide to Describing Cultural Works and Their Images. Chicago: American Library Association, n.d.

Phu, Thy, and Eslpeth H. Brown. Feeling Photography. Durham: Duke University Press, 2014.

Reilly, James M. Care and Identification of $19^{\text {th }}$-century Photographic Prints. Rochester: Image Permanence Institute, 2009.

Tantony, Matt. "Cataloguing Archives in Four Easy Steps." The British Postal Museum \& Archive Blog. https://postalheritage.wordpress.com/2013/09/25/cataloguingarchives-in-four-very-easy-steps/ (Accessed August 1, 2018).

Thompson, Samantha. "How Do Archivists Organize Collections?" Peele Art Gallery Museums \& Archives. August 26, 2015. https://peelarchivesblog.com/2015/08/26/how-do-archivists-organize-collections/ (Accessed August 1, 2018).

\section{SECTION 2}

Rieger, Thomas, Ed. "Technical Guidelines for Digitizing Cultural Heritage Materials: Creation of Raster Image Files." U.S. National Archives and Records Administration, Federal Agencies Digitization Initiative Still Image Working Group, 2016, 1-100. http://www. digitizationguidelines.gov/guidelines/digitizetechnical.html

"Lempel-Ziv-Welch (LZW) Compression." FileFormat.Info. http://www.fileformat.info/mirror/egff/ch09 04.htm (Accessed May 1, 2018).

"Sustainability of Digital Formats: Planning for Library of Congress Collections." Apple Computers: This Month in Business History (Business Reference Services, Library of Congress). September 28, 2009.

https://www.loc.gov/preservation/digital/formats/fdd/fdd000022.shtml (Accessed May 1, 2018.) 


\section{SECTION 3}

Gilliland, Anne J. "Setting the Stage," in Introduction to Metadata, edited by Murtha Baca, 3rd ed. Los Angeles: Getty Publications, 2016, http://www.getty.edu/publications/intrometadata/setting-the-stage/

"DICE Evaluation Parameters." Technical Guidelines for Digitizing Cultural Heritage Materials, The Creation of Raster Image Files. Federal Agencies Digital Guidelines Initiative.

http://www.digitizationguidelines.gov/guidelines/FADGI\%20Federal\%20\%20Agenci es\%20Digital\%20Guidelines\%20Initiative-2016\%20Final rev1.pdf (Accessed April 08, 2018).

\section{SECTION 4}

"Chromaticity Diagrams." Computer Graphics at Stanford University.

https://graphics.stanford.edu/courses/cs178-10/applets/threedgamut.html (Accessed May 25, 2018).

"History of the Very Odd SRGB Color Space." Nine Degrees Below Photography. https://ninedegreesbelow.com/photography/srgb-history.html (Accessed May 25, 2018).

"Adobe RGB (1998) color image encoding". Adobe Digital Imaging Solutions. https://www.adobe.com/digitalimag/adobergb.html (Accessed May 25, 2018).

"2011 Color and Imaging Conference, Part VI: Special Session." RealTime Rendering. http://www.realtimerendering.com/blog/2011-color-and-imaging-conference-part-vispecial-session/ (Accessed May 25, 2018).

Schewe, Jeff. The Digital Negative: Raw Image Processing in Lightroom, Camera Raw, and Photoshop. San Francisco: Peachpit Press, 2016.

\section{GLOSSARY}

"APS-C (APSC)." A Glossary of Digital Photography Terms. B\&H Explora. https://www.bhphotovideo.com/explora/photography/tips-and-solutions/a-glossaryof-digital-photography-terms\#j (Accessed June 08, 2018).

Basic Terminology." Moving Theory into Practice: Digital Imaging Tutorial. Cornell University Library \& Research Department. http://preservationtutorial.library.cornell.edu/intro/intro-04.html (Accessed June 13, 2018). 
"Buffered." Carr McLean Glossary of Archival Terms. https://www.carrmclean.ca/StaticPage.aspx?ContentID=glossaryarch (Accessed June 08, 2018).

"Byte." Computer Hope. April 26, 2017. https://www.computerhope.com/jargon/b/byte.htm (Accessed June 08, 2018).

Berkenfeld, Diane. "Chromatic Aberration | Understanding Chromatic Aberration from Nikon." Nikon | Learn \& Explore. https://www.nikonusa.com/en/learn-andexplore/a/products-and-innovation/chromatic-aberration.html (Accessed June 13, 2018).

"ExFAT." Computer Hope. October 04, 2017. https://www.computerhope.com/jargon/e/exfat.htm (Accessed June 08, 2018).

"IPTC Photo Metadata." IPTC Standard - IPTC. https://iptc.org/standards/photometadata/iptc-standard/ (Accessed June 09, 2018).

"LZW Data Compression." Duke Computer Science. https://www2.cs.duke.edu/csed/curious/compression/lzw.html (Accessed June 08, 2018).

"Microform." Encyclopaedia Brittanica.

https://www.britannica.com/technology/microform\#ref15157 (Accessed June 08, 2018)

"Image-1 Introduction to Digital Images." Introduction to Computing Principles. https://web.stanford.edu/class/cs101/image-1-introduction.html (Accessed June 13, 2018).

"What Is SATA (SerialATA)?" Computer Hope. June 21, 2017. https://www.computerhope.com/jargon/s/sata.htm (Accessed June 13, 2018).

"Tags for TIFF, DNG, and Related Specifications." Sustainability of Digital Formats: Planning for Library of Congress Collections. https://www.loc.gov/preservation/digital/formats/content/tiff tags.shtml (Accessed June 13, 2018). 


\section{GLOSSARY}

Access File ...................... A file formatted for easy and quick retrieval and access (such as a JPEG, PNG, or GIF); Usually saved as a version of a Master or Production Master TIFF file.

Acid-free......................... Materials that have a $\mathrm{pH}$ of 7.0 (neutral) or greater. The presence of acid in a storage material increases the rate of deterioration of the item stored within.

Application

A program or software, often abbreviated as 'app'.

APSC $^{71}$

Advanced Photo System type-C ('classic'); A digital camera sensor that reflects the 'classic' $35 \mathrm{~mm}$ film size $(24 \times 36 \mathrm{~mm})$; also called a 'cropped' sensor. Some digital lenses are only compatible with APSC cameras.

Archival.

Materials, practices, procedures, or digital file format that maintain the greatest possible integrity of a physical record or digital file.

Artifact.

Distortion created in an image by compression or interpolation. Artifacts often appear as areas of blocky chunks of pixels.

AWB

Auto White Balance; A user-selected setting that allows a digital camera to auto-detect the ambient light temperature and compensate internally to produce the most accurate exposure.

Bit

Binary Digit (1 or 0$)$; smallest component of digital information.

71. "APS-C (APSC)." A Glossary of Digital Photography Terms. B\&H Explora.

https://www.bhphotovideo.com/explora/photography/tips-and-solutions/a-glossary-of-digital-photographyterms\#j (Accessed June 08, 2018). 
Bit Depth ${ }^{72}$

In digital imaging, bit depth refers to the number of bits used to characterize each pixel. The number of possible pixel values is calculated by raising 2 (representing the 2 possible values of a bit, 1 or 0 ) to power of the bit number.

1-Bit................. $2^{1}=2$ tones; A purely black and white image with no grey values. Each pixels can only be one of two values, either white or black.

8-Bit................ $2^{8}=256$ tones; A grayscale image with 256 possible values from white to black.

16-Bit................ $2^{16}=65,536$ tones; Either a grayscale or colour image with 65,536 possible values possible for each colour channel (red, green, and blue).

24-Bit................ $2^{24}=16,777,216$ tones; A colour image with approximately 1.7 million values possible for each colour channel (red, green, and blue).

48-Bit............... $2^{48}=281,474,976,710,656$ tones; A colour image with approximately 281 trillion possible values for each colour channel (red, green, and blue).

Buffered $^{73} \ldots \ldots \ldots \ldots \ldots \ldots \ldots \ldots$...................... treated with calcium carbonate $\left(\mathrm{CaCO}_{3}\right)$ to counteract the acidification of materials over time.

Byte $^{74}$

Smallest packet of digital information; 8 bits is equal to 1 byte.

Kilobyte................ K, kB or KB; 1024 bytes

Megabyte. MB or Mb; 1024 kilobytes

Gigabyte GB or $\mathrm{Gb} ; 1024$ megabytes

Terabyte. TB or Tb; 1024 gigabytes

72. "Basic Terminology," Moving Theory into Practice: Digital Imaging Tutorial. Cornell University Library \& Research Department. http://preservationtutorial.library.cornell.edu/intro/intro-04.html (Accessed June 13, 2018).

73. "Buffered," Carr McLean Glossary of Archival Terms. https://www.carrmclean.ca/StaticPage.aspx?ContentID=glossaryarch (Accessed June 08, 2018).

74. "Byte," Computer Hope. April 26, 2017. https://www.computerhope.com/jargon/b/byte.htm (Accessed June 08, 2018). 
Chromatic Aberration ${ }^{75}$............ Distorted colour often appearing as thin lines of either blue or red around edges of high-contrast areas of a digital image. Chromatic Aberration is caused by the different refractive properties of lightwaves, which can't both be completely perfectly focused along a single focal plane by a camera lens. It is sometimes called 'colour fringing' or 'purple fringing' (because when blended or viewed at a distance, the thin lines of red and blue appear to be purple). Professional digital cameras contain built-in features that help minimize chromatic aberration. It can also be remedied in postproduction.

Colour Space......................A mathematical model used to describe a spectrum of possible colours.

Colour Temperature

A description of colour based on a cool to warm spectrum, measured in degrees Kelvin ( $\left.{ }^{\circ} \mathrm{K}\right)$. Blues and greens have a low colour temperature. Oranges and yellows have a high colour temperature.

Compression The process of decreasing a file's size by using various algorithms.

CR2

Canon Raw 2, the proprietary RAW file format generated by Canon Digital Cameras when a digital photograph is taken.

DNG................................. Digital Negative; A non-proprietary raw file format developed by Adobe in the early-2000s.

DPI

Dots Per Inch, referring to the number of ink dots a printer can deposit along one side of one square inch. 300 dpi is 300 dots $\times 300$ dots equaling 90,000 total dots.

75. Diane Berkenfield, "Chromatic Aberration | Understanding Chromatic Aberration from Nikon," Nikon | Learn \& Explore. https://www.nikonusa.com/en/learn-and-explore/a/products-andinnovation/chromatic-aberration.html (Accessed June 13, 2018). 
EXE. Executable File; an application installation file usually associated with Windows operating systems.

exFAT ${ }^{76} \ldots \ldots \ldots \ldots \ldots \ldots \ldots \ldots$ Extended File Allocation Table; a data storage format commonly used with Microsoft and Mac operating systems, as of 2006 and 2008 respectively. Portable hard drives are often formatted as exFAT to allow them to be used with both PCs and Macs.

EXIF...............................Exchangeable Image File Format; A metadata scheme generated by the camera when an image is taken. EXIF metadata records the details of a digital image such as camera serial number, colour space, date taken, lens used, focal length, which balance, ISO, camera firmware, etc.

GIF

Graphic Interchange Format; An uncompressed image file format, GIFs are commonly used for both static and animated web graphics.

Hard drive. A data storage device that can be written to and read by a computer, located either internally or externally attached to the computer.

Interpolation

The computer's process of calculating the data needed to fill in new pixels needed to increase the size of an image.

IPTC $^{77}$ International Press Telecommunications Council; An international organization that creates metadata schema. The two main IPTC photography metadata schema are IPTC-core and IPTC-extension. IPTCcore includes creator, description, keywords, subject, intellectual genre, location, copyright information, etc. IPTC-extension expands upon the IPTC core fields

76. "ExFAT." Computer Hope. October 04, 2017. https://www.computerhope.com/jargon/e/exfat.htm (Accessed June 08, 2018).

77. "IPTC Photo Metadata." IPTC Standard - IPTC. https://iptc.org/standards/photo-metadata/iptcstandard/ (Accessed June 08, 2018). 
and includes fields pertaining to models, organizations, events, etc.

JPEG / JPG ......................... Joint Photographic Experts Group; a lossy digital image format, sometimes written as JPG. Jpeg and Jpg are the same file type, and there is no difference when written in upper or lowercase letters. When first created in the early 90 s, Windows operating systems required a three-letter file extension, so the abbreviation was shortened. Mac operating systems could accommodate four-letter file extensions. Now the use of jpeg versus jpg is usually dependent on the software creating the file.

Jpeg2000 / JP2 ................... A version of the original JPEG format that can be saved as a lossy or lossless file. JPEG2000 are often incompatible with applications and were never widely used.

Kelvin............................. Unit of measurement describing colour temperature ranging from around $1,000 \mathrm{~K}$ (very warm) to $20,000 \mathrm{~K}$ (very cool). Bright sunlight is usually between $5,500 \mathrm{~K}$ and $6,500 \mathrm{~K}$.

Lossless Compression..............A type of compression algorithm that does not discard any data during compression.

Lossy A type of compression algorithm that discards data during compression in order to produce a smaller file.

LZW Compression ${ }^{78} \ldots \ldots \ldots \ldots . . .$. Lempel-Ziv-Welch Compression; Named for its inventors, LZW compression is a method of lossless data compression, meaning no data is discarded, but the file size is reduced.

Megapixel.......................... One million pixels; $A$ unit of measurement to describe the image resolution a digital camera is capable of taking.

78. "LZW Data Compression." Duke Computer Science.

https://www2.cs.duke.edu/csed/curious/compression/lzw.html (Accessed June 08, 2018). 
Microfiche $^{79} \ldots \ldots \ldots \ldots \ldots \ldots \ldots$. Sheets of film $105 \times 148 \mathrm{~cm}$ containing small images, usually of newspaper or book pages, often used for archival or genealogical research. Microfiche is a common method of pre-digital preservation and is still used by institutions today.

Microfilm........................Rolls of film 30.48 m (100 feet) long containing small images, usually of newspaper or book pages, often used for archival or genealogical research. Microfilm is a common method of document preservation.

NEF. Nikon Electronic Format; Nikon's proprietary RAW image file format.

PDF

Portable Document Format; Once converted to a PDF, static documents created in most imaging and wordprocessing programs can be viewed uniformly on any device. PDFs can be either lossy or lossless depending on the compression used.

Pixel.

Picture Element; The smallest unit of measurement in digital optics. In RGB colour spaces, each pixel is a square that is made up of three coloured lights: one red, one blue, and one green. Each light has 255 possible values and the combination and intensity of these lights creates the perceived colour of each pixel. $^{80}$

PNG............................Portable Network Graphic; Created in the mid-1990s as an alternative to GIFs, PNG is an open-source, lossless image file format commonly used online.

PPI. Pixels Per Inch; referring to the number of pixels per inch in a digital image or screen.

79. "Microform." Encyclopaedia Brittanica. https://www.britannica.com/technology/microform\#ref15157 (Accessed June 09, 2018).

80. "Image-1 Introduction to Digital Images." Introduction to Computing Principles.

https://web.stanford.edu/class/cs101/image-1-introduction.html (Accessed June 13, 2018). 
PSD ................................. Photoshop Document; A proprietary file type used by Adobe Photoshop that can contain layers, masks, adjustments that are preserved when the file is saved (i.e. the file is not compressed or flattened like a jpeg).

RAM

Random Access Memory; memory storage within a computer that is used for the temporary storing and retrieving of information while a computer is powered on. No memory is permanently stored in RAM. The more RAM that is in a computer, the more tasks it can do at once since it has more space to temporarily store data needed for the timely functioning of applications and user commands. Most computers come with between $4 \mathrm{~GB}$ and $256 \mathrm{~GB}$ of RAM.

RAW

RAW files are image files captured in most digital single lens reflex (DSLR) cameras. They are uncompressed and unedited, and contain the maximum amount of data the camera can capture in an exposure. Because they contain the most amount of data, it is best to set your camera to shoot RAW files, import and edit in Lightroom, and then export as TIFFs or JPEGs.

Recto............................ The front side of a piece of paper, usually the page of a book.

Resolution........................ Measure in pixels or dots per inch, resolution refers to the quality of an image either displayed on a monitor or produced by a printer. The more dots or pixels per inch (or centimeter) the greater the resolution.

ROM...............................Read-Only Memory; memory in a computer that contains the code and firmware necessary for the computer to boot up and function. It can only be read, not written to, meaning that you cannot save data on, or edit ROM. 
SATA $^{81}$

Serial Advanced Technology Attachment; A highspeed wired connection which, in the context of hard drives, SATA refers to a hard drive containing a spinning disk. SATA can also be used in Solid-state hard drive technology, but more commonly SSDs use a faster mSATA (mini-SATA) or M.2 connection.

SSD

Solid-State Drive; A hard drive that contains no moving parts, allowing it to perform faster and more quietly, while requiring less power than spinning hard drives.

TIFF $^{82}$

Tagged Image File Format; A lossless image file format. Tags refer to metadata embedded within the image file, which can range from data generated during the file's creation to information added in postprocessing.

Verso The backside of a piece of paper, usually the page of a book.

White Balance

Determining accurate representations of white under various lighting conditions. Digital cameras and imaging software allow the user to either select AWB (auto white balance) or to set a custom white balance temperature, measured in degrees Kelvin.

XMP

Extensible Metadata Platform; A standardized metadata schema that can be embedded in image files, or saved as sidecar files (files that live outside of an image file, but whose data links back to that image file).

81. "What Is SATA (SerialATA)?" Computer Hope. June 21, 2017. https://www.computerhope.com/jargon/s/sata.htm (Accessed June 13, 2018).

82. "Tags for TIFF, DNG, and Related Specifications." Sustainability of Digital Formats: Planning for Library of Congress Collections. https://www.loc.gov/preservation/digital/formats/content/tiff_tags.shtml (Accessed June 13, 2018). 


\section{CONCLUSION}

Digitization in itself is not difficult but digitizing in an archival manner requires a few extra steps and attention to detail to ensure that certain standards-standards that have been established to ensure the longevity and quality of digital files-are met. Some of these steps and standards, especially when presented by institutions and organizations whose primary audience are other industry professionals, can be intimidating, confusing, and discouraging to anyone without archiving or digital imaging training who wishes to learn how to digitize their own personal collection. This divide represents a disparity that is ultimately detrimental to the preservation of cultural and historical materials that are not housed in the collections of museums and archives. However, the way in which institutions collect may also change; where once focus was placed on the procurement of physical items for a collection, some institutions have begun accepting—or would like to accept—digital donations, consisting of both born digital images, such as those taken with a digital camera, and digital surrogates of physical records. Thus, the archival digitization-digitization that meets certain standards - of personal archives is not only important for the families whose history is remembered in those objects, but will be vital for the expansion of larger institutional collections that already have an established network of objects and information available to the public. So much history is told in the quotidian objects and records held in small personal collections, and currently this information is largely absent in institutional collections. 